Supporting Information

\title{
A Dual-Emissive Phosphorescent Polymeric Probe for Exploring Drug-Induced Liver Injury via Imaging of Peroxynitrite Elevation in Vivo
}

Zejing Chen, ${ }^{\dagger,}$ Xiangchun Meng,,$^{\dagger}$ Liang Zou,,$^{\dagger}$ Menglong Zhao, ${ }^{\dagger}$ Shujuan Liu, ${ }^{*},+$ Peng Tao, ${ }^{\dagger}$ Jiayang Jiang, ${ }^{\dagger}$ and Qiang Zhao*,广

†Key Laboratory for Organic Electronics and Information Displays \& Jiangsu Key Laboratory for Biosensors, Institute of Advanced Materials (IAM), Nanjing University of Posts \& Telecommunications, 9 Wenyuan Road, Nanjing 210023, P. R. China.

†iangxi Key Laboratory for Nano-Biomaterials, Institute of Advanced Materials (IAM), East China Jiaotong University, 808 Shuanggang East Main Street, Nanchang 330013, P. R. China.

*Corresponding Authors: E-mail: iamsjliu@njupt.edu.cn, iamqzhao@njupt.edu.cn. 


\section{Table of Contents}

\section{Methods}

\subsection{General experimental information}

\subsection{Sources for different ROS/RNS}

\subsection{Theoretical calculation}

\subsection{Cell culture and MTT assay}

\subsection{Photoluminescence imaging}

\subsection{Histology and H\&E staining}

\section{Synthesis}

Scheme S1. Synthetic procedure of complex Ir1, Ir2, Ir3 and Ir4.

Scheme S2. Synthetic procedure of complex Ir1*, Ir2*, Ir3* and Ir4*.

Scheme S3. Synthetic procedure of complex Ir5 and Ir6.

Scheme S4. Synthetic procedure of P-ONOO.

\section{Supplementary Tables and Figures}

\subsection{Tables}

Table S1. Photophysical data for complexes and polymers.

Table S2. Quantum efficiencies of iridium(III) complex probes in different viscous solution.

Table S3. Calculated energy levels of the lowest triplet state for $\mathrm{N}^{\wedge} \mathrm{N}$ ligands.

Table S4. Calculated important molecular orbits of complexes.

Table S5. Calculated phosphorescent emission of complexes.

\subsection{Figures}

Figure S1. Normalized absorption spectra of phosphorescent probes and model complexes.

Figure S2. Emission spectra of Ir1, Ir2, Ir3 and Ir4 upon addition of $\mathrm{ONOO}^{-}$and corresponding model complexes Ir1*, Ir2*, Ir3* and Ir4*.

Figure S3. The MALDI-TOF-MS spectra of iridium(III) complex probes (Ir1, Ir2 and Ir3) and iridium(III) complex probes incubated with excessive ONOO- 
Figure S4. The MALDI-TOF-MS spectra of iridium(III) complex probe Ir4, probe Ir4 incubated with excessive $\mathrm{ONOO}^{-}$and model iridium(III) complex Ir4*.

Figure S5. The ${ }^{1} \mathrm{H}$ NMR spectra of iridium(III) complex probe Ir4, main product of Ir4 reacting with excessive $\mathrm{ONOO}^{-}$and $\mathbf{I r} \mathbf{4}^{*}$.

Figure S6. Proposed mechanisms of reactions between iridium(III) complex probes and $\mathrm{ONOO}^{-}$.

Figure S7. The MALDI-TOF-MS spectra of Ir5, Ir6 and samples incubated with excessive ONOO-

Figure S8. Time course in the detection of $\mathrm{ONOO}^{-}$with Ir4, Ir5 and Ir6.

Figure S9. Normalized absorption and emission spectra of phosphorescent iridium complex Ir7.

Figure S10. Normalized absorption and emission spectra of P-ONOO.

Figure S11. Effects of concentration, ionic strength, viscosity and freshness on the phosphorescence ratio of P-ONOO.

Figure S12. MTT assay about cytotoxicity of P-ONOO on RAW264.7 cells and HepG2 cells.

Figure S13. The CLSM images, ratiometric photoluminescence images and PLIM images of living RAW264.7 cells labeled with P-ONOO under different exogenous stimulations.

Figure S14. The CLSM images, ratiometric photoluminescence images and PLIM images of living RAW264.7 cells labeled with P-ONOO under different endogenous stimulations.

Figure S15. The CLSM images, ratiometric photoluminescence images and PLIM images of living HepG2 cells labeled with P-ONOO under different endogenous stimulations.

Figure S16. The DCFH-stained experiments of HepG2 cells pretreated with KTZ.

Figure S17. The JC-1 stained experiments of HepG2 cells pretreated with KTZ.

Figure S18. P-ONOO for endogenous $\mathrm{ONOO}^{-}$detection in living HL 7702 cells by ratiometric photoluminescence imaging.

Figure S19. Ex vivo images of major organs after sacrificing mice at different time after intravenously injected with P-ONOO.

Figure S20. Photoluminescence imaging of mice treated with normal saline.

Figure S21. Photoluminescence imaging of mice pretreated with NAC.

Figure S22. Typical H\&E staining images of the liver of mice treated under various conditions.

Figures S23-32 MALDI-TOF mass spectroscopic characterization of related complexes.

Figure S33-75 NMR spectroscopic characterization of related complexes and other chemical intermediates.

\section{References}




\section{Methods}

\subsection{General experimental information}

All reagents and chemicals were procured from commercial sources and used without further purification unless otherwise noted. All solvents were of analytical grade and purified according to standard procedures. The detailed synthesis of complexes and relative ligands can be found in the Scheme S1-4. Cell culture reagents and fetal bovine serum (FBS) were purchased from Gibcco. The ${ }^{1} \mathrm{H}$ and ${ }^{13} \mathrm{C}$ NMR spectra were recorded on a Bruker Ultra Shield Plus $400 \mathrm{MHz}$ NMR instrument at $298 \mathrm{~K}$ using deuterated solvents. Chemical shifts are given in ppm, and are referenced against external $\mathrm{Me}_{4} \mathrm{Si}\left({ }^{1} \mathrm{H},{ }^{13} \mathrm{C}\right)$. Mass spectra were obtained on a Bruker autoflex matrix-assisted laser desorption ionization time-of-flight (MALDI-TOF) mass spectrometer. The UV-visible absorption spectra were obtained with a Shimadzu UV-3600 UV-VIS-NIR spectrophotometer. Photoluminescence spectra were measured on an Edinburgh FL 920 spectrophotometer. Excited-state lifetime studies were performed with an Edinburgh LFS-920 spectrometer with a hydrogen-filled excitation source. The data were analyzed by using a software package provided by Edinburgh Instruments. The absolute quantum yields of the complexes were determined through an absolute method by employing an integrating sphere. The methyl thiazolyltetrazolium assay was performed by a Power Wave XS/XS2 microplate spectrophotometer. The photoluminescence imaging and time-resolved photoluminescence imaging of cells were carried out on an Olympus FV1000 confocal laser scanning microscope. The PLIM setup is integrated with the same Olympus FV1000 confocal laser scanning microscope. The lifetime values were calculated with professional software provided by PicoQuant Company. The photoluminescence imaging of mice was carried out on living animal photoluminescence imaging system (IVIS Lumina K Series III In Vivo Imaging, PerkinElmer). The time-resolved photoluminescence imaging of mice was carried out on the Becker \& Hick1 
GmbH DCS-120 confocal scanning PLIM system and the lifetime data were calculated by related professional software provided by Becker \& Hickl GmbH company.

\subsection{Sources for different ROS/RNS}

For $\mathrm{ONOO}^{-}$, it was obtained by preparing peroxynitrous acid $(\mathrm{ONOOH})$ and quenching ONOOH with alkaline solution. Briefly, hydrochloric acid (0.6 M) was mixed into a solution containing sodium nitrite $(0.6 \mathrm{M})$ and hydrogen peroxide $(0.7 \mathrm{M})$ in an ice bath. Within following 1-2 s, sodium hydroxide (1.5 M) was added into above mixture to make the solution alkaline. Then, the mixture was filtered through a short column of manganese dioxide to remove the excess hydrogen peroxide. The filtrate was divided into small aliquots and stored at $-80{ }^{\circ} \mathrm{C}$. The concentration of peroxynitrite in each aliquot was determined before use by measuring absorbance at $302 \mathrm{~nm}$. The extinction coefficient of peroxynitrite solution in $0.1 \mathrm{M} \mathrm{NaOH}$ is $1,670 \mathrm{M}^{-1} \mathrm{~cm}^{-1}$ at $302 \mathrm{~nm}$. An appropriate amount was utilized to provide $\mathrm{ONOO}^{-}$. For $\mathrm{H}_{2} \mathrm{O}_{2}$ and $\mathrm{NaClO}$, they were available from commercial sources and a freshly stock solution was prepared before use. For superoxide radical anion $\left(\mathrm{O}_{2}{ }^{\circ-}\right)$, an appropriate amount of solid potassium superoxide was suspended in DMSO by sonication and used as $\mathrm{O}_{2}{ }^{\bullet-}$ source. For nitric oxide (NO), it was prepared by adding $\mathrm{H}_{2} \mathrm{SO}_{4}$ solution $(3.6 \mathrm{M})$ into a stirred solution of $\mathrm{NaNO}_{2}$ $(7.3 \mathrm{M})$ dropwise. Adequate amounts of gas produced in the reaction passed through $\mathrm{NaOH}$ solution (2 M) first and then deionized $\mathrm{H}_{2} \mathrm{O}$ to acquire a saturated $\mathrm{NO}$ solution (about $2.0 \mathrm{mM}$ ). For hydroxy radical $(\cdot \mathrm{OH})$, it was generated by Fenton reaction between ferrous chloride and hydrogen peroxide. The concentration of ' $\mathrm{OH}$ was equal to the $\mathrm{Fe}(\mathrm{II})$ concentration. For ROO', an appropriate amount of $\mathrm{tBuOOH}$ was dissolved in deionized $\mathrm{H}_{2} \mathrm{O}$ as freshly solution for use. For ${ }^{1} \mathrm{O}_{2}$, equal aliquots of $\mathrm{NaMoO}_{4}(20 \mathrm{mM})$ and $\mathrm{H}_{2} \mathrm{O}_{2}(20 \mathrm{mM})$ were mixed in PBS to yield ${ }^{1} \mathrm{O}_{2}(10 \mathrm{mM})$. For GSH, Cys, Hcy, NaHS and $\mathrm{Na}_{2} \mathrm{~S}_{2} \mathrm{O}_{3}$, they were procured from commercial sources and added into the solution directly. 


\subsection{Theoretical calculation}

The ground-state and the lowest-lying triplet excited-state geometries were optimized by density functional theory (DFT) with Becke's LYP (B3LYP) exchange-correlation functional and the unrestricted B3LYP (UB3LYP) approach, respectively. On the basis of ground- and excited-state optimization, the time-dependent DFT (TDDFT) approach associated with the polarized continuum model (PCM) in dichloromethane media was carried out to obtain the vertical excitation energies of triplet $(\mathrm{Tn})$ states. The calculation was performed using the Gaussian 09 suite of programs. The LANL2DZ basis set was used to treat the iridium atom, whereas the $6-31 \mathrm{G}^{*}$ basis set was used to treat all other atoms. The contours of important molecular orbitals (HOMOs and LUMOs) were plotted.

\subsection{Cell culture and MTT assay}

The RAW 264.7 and HepG2 cell lines were purchased from the Institute of Biochemistry and Cell Biology, SIBS, CAS (China). RAW 264.7 cells were incubated in Roswell Park Memorial Institute (RPMI 1640) supplemented with $10 \%$ fetal bovine serum (FBS), $100 \mathrm{mg} \mathrm{mL}^{-1}$ streptomycin and $100 \mathrm{U} \mathrm{mL}^{-1}$ penicillin in a humidified incubator at $37^{\circ} \mathrm{C}$ with $5 \% \mathrm{CO}_{2}$. $\mathrm{HepG} 2$ cells were incubated in Dulbecco's modified Eagle's medium (DMEM) supplemented with 10\% fetal bovine serum (FBS), $100 \mathrm{mg} \mathrm{mL}^{-1}$ streptomycin and $100 \mathrm{U} \mathrm{mL}^{-1}$ penicillin in a humidified incubator at $37^{\circ} \mathrm{C}$ with $5 \% \mathrm{CO}_{2}$. The in vitro cytotoxicity toward cells was measured using the methyl thiazolyl tetrazolium assay. Briefly, cells growing in log phase were seeded into 96-well cell culture plate at $1 \times 10^{4} /$ well. P-ONOO was added to the wells of the treatment group at concentrations of 10, 50, 100, 200 and $400 \mu \mathrm{g} \mathrm{mL}-1$. The cells were incubated for $24 \mathrm{~h}$ at $25^{\circ} \mathrm{C}$ under $5 \% \mathrm{CO}_{2}$. The MTT (5 mg mL-1) in PBS solution was added to each well, and incubated for another $4 \mathrm{~h}$. After removal of the culture solution, $200 \mu \mathrm{L}$ DMSO was added to each well and then shaked for 10 min at shaking table. An enzyme-linked immunosorbent assay (ELISA) reader (BioTek Instruments, Rower Wave XS2) was used to measure the OD570 (Absorbance 
value) of each well referenced at $570 \mathrm{~nm}$. The following formula was used to calculate the viability of cell growth:

Viability $(\%)=($ mean of absorbance value of treatment group / mean of absorbance value of control) $\times 100$.

\subsection{Photoluminescence imaging}

RAW 264.7 or HepG2 cells used for imaging were first incubated in culture dishes until their adherence. The cells were washed with PBS three times and then incubated with P-ONOO (100 $\mu \mathrm{g} \mathrm{mL}-1$ ) in serum-free culture medium for 2 hours at $37^{\circ} \mathrm{C}$ and $5 \% \mathrm{CO}_{2}$. Cell imaging experiments were then performed after the cells were washed and covered with $1 \mathrm{~mL}$ PBS in the culture dishes for imaging.

Photoluminescence imaging was performed with an Olympus FV1000 confocal laser scanning microscope, a 40× objective lens for cells. RAW 264.7 or HepG2 cells containing the $\mathrm{P}-\mathrm{ONOO}$ were excited at $515 \mathrm{~nm}$ with a semiconductor laser, and the emission was measured according to the spectral data. The images were accomplished using the software package provided by Olympus instruments.

Photoluminescence lifetime imaging microscopy and time-gated photoluminescence imaging techniques for cells were adopted on the platform afforded by Olympus FV1000 confocal laser scanning microscope and PicoQuant Company. The objective lens was $40 \times$ and the frequency was $0.5 \mathrm{MHz}$. Phosphorescence was excited with $405 \mathrm{~nm}$ light, the emission was collected through bandpass filter $(600 \pm 20 \mathrm{~nm})$ or longpass filter $(\geq 650 \mathrm{~nm})$. The related calculations of the data were carried out with the software provided by PicoQuant.

All the mice experiments were carried out in accordance with the relevant laws and the guidelines of Institutional Animal Care and Use Committee. The female nude mice (SPF, 8-10 weeks) and ICR mice (8-10 weeks) were purchased from Comparative Medicine Centre of Yangzhou University. For inflammation model, saline was injected on left leg of mice and LPS 
$\left(200 \mu \mathrm{L}, 1 \mathrm{mg} \mathrm{mL}^{-1}\right)$ was injected on right leg of mice. After $12 \mathrm{~h}, \mathrm{P}-\mathrm{ONOO}\left(50 \mu \mathrm{L}, 5 \mathrm{mg} \mathrm{mL}^{-1}\right)$ were injected subcutaneously on left and right leg of mice. After $1 \mathrm{~h}$, the mice were anesthetized by isoflurane for imaging. For drug induced liver injury model, ICR mice ( $\sim 30 \mathrm{~g})$ were fasted for $12 \mathrm{~h}$ to avoid the possible fluorescence interference. $300 \mu \mathrm{L}$ saline containing APAP (300 $\left.\mathrm{mg} \mathrm{kg}^{-1}\right)$ was injected intraperitoneally and P-ONOO $\left(5 \mathrm{mg} \mathrm{mL}^{-1}\right)$ in $100 \mu \mathrm{L}$ saline was injected through the tailvein. For the remediation investigation, $300 \mu \mathrm{L}$ saline containing NAC $(300 \mathrm{mg}$ $\mathrm{kg}^{-1}$ ) was injected intraperitoneally $1 \mathrm{~h}$ before drug administration. Then, all the mice were anaesthetized and performed surgical procedure to expose the liver. The liver was imaged every 5 min with excitation at $510 \mathrm{~nm}$ and the emission signals at $610 \pm 20 \mathrm{~nm}$ and $710 \pm 20 \mathrm{~nm}$ were collected, respectively.

Photoluminescence lifetime imaging microscopy and time-gated photoluminescence imaging techniques for mice was adopted on the platform afforded by Becker \& Hickl GmbH DCS-120 confocal scanning PLIM system and phosphorescence was excited with $515 \mathrm{~nm}$ light, the emission was collected through a bandpass filters $(562 \pm 20 \mathrm{~nm}$ or $710 \pm 25 \mathrm{~nm})$. The related calculations of the data were carried out with the software SPC imaging provided by Becker \& Hickl GmbH.

\subsection{Histology and H\&E staining}

ICR mice $(\sim 30 \mathrm{~g})$ were fasted for $12 \mathrm{~h}$ to avoid the possible fluorescence interference. The mice were injected intraperitoneally with $300 \mu \mathrm{L}$ saline containing APAP (300 mg kg-1) or saline. After $4 \mathrm{~h}$, the mice were dissected to isolate the livers, which were then fixed with $4 \%$ paraformaldehyde (PFA) for $12 \mathrm{~h}$. In other drug-treated groups, mice were given intragastric gavage KTZ (160 mg kg-1). After $4 \mathrm{~h}, 12 \mathrm{~h}$ and $24 \mathrm{~h}$, respectively, the mice were dissected to isolate the livers, which were then fixed with 4\% PFA for $12 \mathrm{~h}$. For the remediation investigation, $300 \mu \mathrm{L}$ saline containing NAC (300 mg kg-1) was injected intraperitoneally $1 \mathrm{~h}$ before drug administration. After $36 \mathrm{~h}$, respectively, the mice were dissected to isolate the livers, 
which were then fixed with $4 \%$ PFA for $12 \mathrm{~h}$. H\&E staining samples were prepared from the mice stained by hematoxylin and eosin under standard protocols. The experiments were carried out by Servicebio company. 


\section{Synthesis}

All reagents and chemicals were procured from commercial sources and used without further purification unless otherwise noted. All solvents were of analytical grade and purified according to standard procedures. ${ }^{1}$

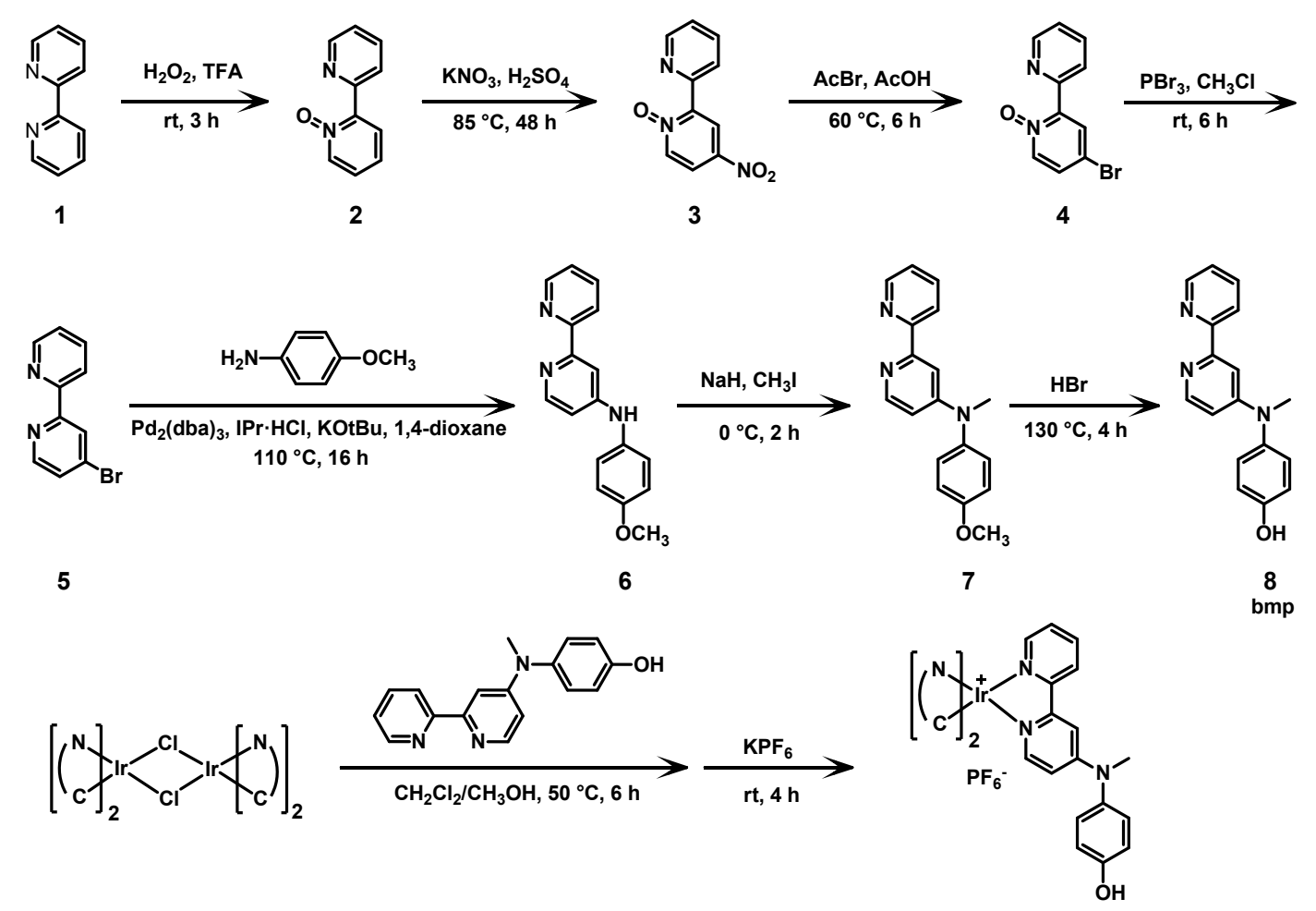

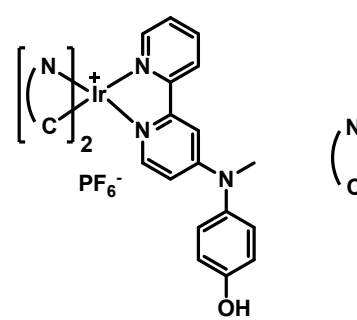<smiles>Fc1ccc(-c2ccc[nH]2)c(F)c1</smiles>

Ir1<smiles>c1c[nH]c(-c2ccc3ccccc3n2)c1</smiles>

Ir2<smiles>C1=Cc2nc3c(nc2=C1)C(c1cccs1)=CC=3</smiles>

Ir3

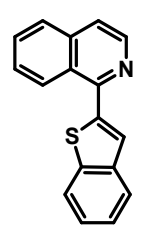

Ir4

$\left[\operatorname{lr}(\mathrm{fpp})_{2}(\mathrm{bmp})\right]^{+} \mathrm{PF}_{6^{-}} \quad\left[\operatorname{lr}(\mathrm{pql})_{2}(\mathrm{bmp})\right]^{+} \mathrm{PF}_{6^{-}} \quad\left[\operatorname{lr}(\mathrm{tpq})_{2}\left(\mathrm{bmp}^{-}\right]^{+} \mathrm{PF}_{6^{-}} \quad\left[\operatorname{lr}(\mathrm{btq})_{2}(\mathrm{bmp})\right]^{+} \mathrm{PF}_{6^{-}}\right.$

Scheme S1 Synthetic procedure of complex Ir1, Ir2, Ir3 and Ir4

4-bromo-2,2'-bipyridine (5). This compound was prepared according to the literature procedure. ${ }^{2}$

$\mathrm{N}$-(4-methoxyphenyl)-[2,2'-bipyridin]-4-amine (6). An oven-dried Schlenk tube was charged with 4-bromo-2,2'-bipyridine (2000 mg, $8.51 \mathrm{mmol})$, 4-methoxyaniline (1260 $\mathrm{mg}, 10.23$ mmol), $\mathrm{Pd}_{2}(\mathrm{dba})_{3}$ (395 $\mathrm{mg}, 0.43 \mathrm{mmol}$ ), 1,3-bis(2,6-diisopropylphenyl)imidazol-2-ylidene (IPrHCl) (370 mg, $0.87 \mathrm{mmol})$ and $\mathrm{Cs}_{2} \mathrm{CO}_{3}(6.91 \mathrm{~g}, 21.28 \mathrm{mmol})$, and flushed with $\mathrm{N}_{2}$ gas for $30 \mathrm{~min}$. Then anhydrous 1,4-dioxane $(20 \mathrm{~mL})$ was introduced. The resulting mixture was first stirred under $\mathrm{N}_{2}$ at room temperature for $30 \mathrm{~min}$ and then at $100{ }^{\circ} \mathrm{C}$ for $20 \mathrm{~h}$. The reaction mixture was allowed to cool to room temperature, diluted with $\mathrm{CH}_{2} \mathrm{Cl}_{2}$ and filtered. The filter cake was washed with $\mathrm{CH}_{2} \mathrm{Cl}_{2}$. The filtrate was then concentrated and the residue was purified by silica gel chromatograph using dichloromethane/methanol $(\mathrm{v} / \mathrm{v}=50 / 1)$ to give milky white solid. Yield: 32\%. ${ }^{1} \mathrm{H}$ NMR (400 MHz, $\left.\mathrm{CDCl}_{3}\right) \delta(\mathrm{ppm}): 8.63$ (ddd, $J=4.8 \mathrm{~Hz}, 1.6 \mathrm{~Hz}, 0.8 \mathrm{~Hz}$, 
$1 \mathrm{H}), 8.34(\mathrm{dt}, J=8.0 \mathrm{~Hz}, 0.8 \mathrm{~Hz}, 1 \mathrm{H}), 8.31(\mathrm{~d}, J=5.6 \mathrm{~Hz}, 1 \mathrm{H}), 7.82-7.77(\mathrm{~m}, 2 \mathrm{H}), 7.29$ (ddd, $J=4.8 \mathrm{~Hz}, 1.6 \mathrm{~Hz}, 0.8 \mathrm{~Hz}, 1 \mathrm{H}), 7.20-7.16(\mathrm{~m}, 2 \mathrm{H}), 6.95-6.90(\mathrm{~m}, 2 \mathrm{H}), 6.70$ (dd, $J=5.6 \mathrm{~Hz}, 2.4$ $\mathrm{Hz}, 1 \mathrm{H}), 6.02(\mathrm{~s}, 1 \mathrm{H}), 3.83(\mathrm{~s}, 3 \mathrm{H}) .{ }^{13} \mathrm{C} \mathrm{NMR}\left(100 \mathrm{MHz}, \mathrm{CDCl}_{3}\right) \delta(\mathrm{ppm}): 157.1,156.8,156.4$, $152.9,149.9,149.0,136.9,132.3,125.2,123.6,121.2,114.8,108.2,106.5,77.2,55.6$.

$\mathrm{N}$-(4-methoxyphenyl)-N-methyl-[2,2'-bipyridin]-4-amine (7). Compound 6 (700 mg, 2.53 $\mathrm{mmol})$ and $\mathrm{NaH}(60 \%$ in oil, $120 \mathrm{mg}, 3.04 \mathrm{mmol})$ was cooled to $0{ }^{\circ} \mathrm{C}$ in an ice bath under $\mathrm{N}_{2}$. Then, anhydrous $N, N$-dimethylformamide $(8 \mathrm{~mL})$ was added into the mixture. After 30 minutes of stir at $0{ }^{\circ} \mathrm{C}, \mathrm{CH}_{3} \mathrm{I}(173 \mu \mathrm{L}, 2.78 \mathrm{mmol})$ was added. Immediately following addition, the mixture was warmed to ambient temperature slowly (by removing the ice bath). The reaction was monitored by TLC analysis till the disappearance of compound 6 (ca. $2 \mathrm{~h}$ ). At that point, $\mathrm{H}_{2} \mathrm{O}(2.0 \mathrm{~mL})$ was added rapidly to quench the reaction and the mixture was diluted with $\mathrm{CH}_{2} \mathrm{Cl}_{2}(20.0 \mathrm{~mL})$. The biphasic mixture was then transferred to a separatory funnel and the organic layer was washed sequentially with $\mathrm{H}_{2} \mathrm{O}$ and brine, dried over anhydrous $\mathrm{Na}_{2} \mathrm{SO}_{4}$, filtered, and concentrated under reduced pressure. The residue was purified by silica gel chromatograph using dichloromethane/methanol $(\mathrm{v} / \mathrm{v}=150 / 1$,$) to give light brown solid. Yield:$ 89\%. ${ }^{1} \mathrm{H}$ NMR $\left(400 \mathrm{MHz}, \mathrm{CDCl}_{3}\right) \delta(\mathrm{ppm}): 8.63(\mathrm{~d}, J=4.8 \mathrm{~Hz}, 1 \mathrm{H}), 8.33(\mathrm{~d}, J=8.0 \mathrm{~Hz}, 1 \mathrm{H})$, $8.23(\mathrm{~d}, J=5.6 \mathrm{~Hz}, 1 \mathrm{H}), 7.77(\mathrm{dt}, J=7.6 \mathrm{~Hz}, 1.6 \mathrm{~Hz}, 1 \mathrm{H}), 7.71(\mathrm{~d}, J=2.4 \mathrm{~Hz}, 1 \mathrm{H}), 7.28-7.24$ $(\mathrm{m}, 1 \mathrm{H}), 7.18-7.14(\mathrm{~m}, 2 \mathrm{H}), 6.98-6.94(\mathrm{~m}, 2 \mathrm{H}), 6.46(\mathrm{dd}, J=5.6 \mathrm{~Hz}, 2.4 \mathrm{~Hz}, 1 \mathrm{H}), 3.84(\mathrm{~s}, 3 \mathrm{H})$, $3.38(\mathrm{~s}, 3 \mathrm{H}) .{ }^{13} \mathrm{C} \mathrm{NMR}\left(100 \mathrm{MHz}, \mathrm{CDCl}_{3}\right) \delta(\mathrm{ppm}): 158.0,156.8,156.4,155.3,149.01,148.96$, $139.2,136.8,128.2,123.4,121.3,115.2,108.4,104.8,55.5,39.9$.

4-([2,2'-bipyridin]-4-yl(methyl)amino)phenol (8). Compound 7 (600 mg, $2.06 \mathrm{mmol})$ was dissolved in hydrobromic acid $(5.0 \mathrm{~mL})$ and the solution was stirred under $\mathrm{N}_{2}$ at $130{ }^{\circ} \mathrm{C}$. The reaction was monitored by TLC analysis till the disappearance of compound 8 (ca. $4 \mathrm{~h}$ ). and allowed to cool to room temperature. Then the mixture was neutralized with saturated sodium carbonate solution and diluted with ethyl acetate. The biphasic mixture was then transferred to a separatory funnel and the aqueous layer was washed sequentially with ethyl acetate. The organic layer was collected and dried over anhydrous $\mathrm{Na}_{2} \mathrm{SO}_{4}$, filtered, and concentrated under reduced pressure. The residue was purified by silica gel chromatograph using dichloromethane/methanol $(\mathrm{v} / \mathrm{v}=50 / 1$,$) to give light brown solid. Yield: 83 \%$. ${ }^{1} \mathrm{H}$ NMR (300 MHz, DMSO-d $\left.{ }_{6}\right) \delta(\mathrm{ppm}): 9.76(\mathrm{~s}, 1 \mathrm{H}), 8.56(\mathrm{~d}, J=4.4 \mathrm{~Hz}, 1 \mathrm{H}), 8.31(\mathrm{~d}, J=8.0 \mathrm{~Hz}, 1 \mathrm{H}), 8.21$ $(\mathrm{d}, J=6.0 \mathrm{~Hz}, 1 \mathrm{H}), 7.85(\mathrm{dt}, J=7.6 \mathrm{~Hz}, 1.6 \mathrm{~Hz}, 1 \mathrm{H}), 7.55(\mathrm{~d}, J=2.4 \mathrm{~Hz}, 1 \mathrm{H}), 7.35$ (ddd, $J=$ 7.2 Hz, 4.8 Hz, 0.8 Hz, 1H), 7.10-7.06 (m, 2H), 6.87-6.83 (m, 2H), 6.57 (dd, $J=6.0 \mathrm{~Hz}, 2.8$ $\mathrm{Hz}, 1 \mathrm{H}), 3.26(\mathrm{~s}, 3 \mathrm{H}) .{ }^{13} \mathrm{C}$ NMR $\left(100 \mathrm{MHz}, \mathrm{DMSO}-\mathrm{d}_{6}\right) \delta(\mathrm{ppm}): 156.5,156.3,155.5,155.3$, $149.8,149.4,137.6,137.4,128.6,124.2,121.0,117.1,108.2,104.6,40.2$.

$\left(C^{\wedge} N\right)_{2} \operatorname{Ir}(\mu-C l)_{2} \operatorname{Ir}\left(C^{\wedge} N\right)_{2}$ The cyclometalated iridium(III) chloro-bridged dimers were synthesized according to the Nonoyama route by refluxing $\mathrm{IrCl}_{3} \cdot 3 \mathrm{H}_{2} \mathrm{O}$ with $2.0-2.5$ equiv. of cyclometalating ligand $\left(\mathrm{HC}^{\wedge} \mathrm{N}\right)$ in a $3: 1(\mathrm{~V}: \mathrm{V})$ mixture of 2-ethoxyethanol and water. ${ }^{3}$ Here, the $\left[\operatorname{Ir}(\mathrm{fpp})_{2} \mathrm{Cl}\right]_{2}$ was taken as an example. A mixture of 2-ethoxyethanol and water $(\mathrm{v} / \mathrm{v}=3 / 1)$ was added to a flask containing $\mathrm{IrCl}_{3} \cdot 3 \mathrm{H}_{2} \mathrm{O}(420 \mathrm{mg}, 1.19 \mathrm{mmol})$ and 2-(2,4difluorophenyl)pyridine $(510 \mathrm{mg}, 2.67 \mathrm{mmol})$. The mixture was refluxed under nitrogen at 110 ${ }^{\circ} \mathrm{C}$ for $24 \mathrm{~h}$. After cooling, the yellow-green solid precipitate was filtered to give crude cyclometalated $\operatorname{Ir}(\mathrm{III})$ chloro-bridged dimer.

$\left[\mathbf{I r}(\boldsymbol{f p p})_{2}(\boldsymbol{b m p})\right]^{+} \boldsymbol{P F}_{6}{ }^{-}$(Ir1). A mixture of cyclometalated iridium(III) chloro-bridged dimer $\left[\operatorname{Ir}(\mathrm{fpp})_{2} \mathrm{Cl}\right]_{2}(200 \mathrm{mg}, 0.16 \mathrm{mmol})$ and 4-([2,2'-bipyridin]-4-yl(methyl)amino)phenol (100 mg, $0.36 \mathrm{mmol})$ in solution of dichloromethane/methanol $\left(\mathrm{v} / \mathrm{v}=2 / 1\right.$, ) was heated at $50{ }^{\circ} \mathrm{C}$ under nitrogen for $6 \mathrm{~h}$. The mixture was then cooled to room temperature, and stirred for another $4 \mathrm{~h}$ after $\mathrm{KPF}_{6}(600 \mathrm{mg}, 3.26 \mathrm{mmol})$ was added to the solution. Then, the mixture was filtered and evaporated under reduced pressure. The residue was purified by silica gel chromatograph using 
dichloromethane/methanol $(\mathrm{v} / \mathrm{v}=100 / 1)$ to give the yellow-green solid, complex Ir1. Yield: 53\%. ${ }^{1} \mathrm{H}$ NMR (400 MHz, $\left.\mathrm{CDCl}_{3}\right) \delta(\mathrm{ppm}): 8.25(\mathrm{~d}, J=8.8 \mathrm{~Hz}, 2 \mathrm{H}), 8.05$ (dd, $J=7.6 \mathrm{~Hz}, 7.6$ $\mathrm{Hz}, 1 \mathrm{H}), 7.88(\mathrm{~d}, J=5.2 \mathrm{~Hz}, 1 \mathrm{H}), 7.77(\mathrm{dd}, J=7.6 \mathrm{~Hz}, 7.6 \mathrm{~Hz}, 2 \mathrm{H}), 7.70(\mathrm{~d}, J=5.6 \mathrm{~Hz}, 1 \mathrm{H})$, $7.48(\mathrm{~d}, J=5.6 \mathrm{~Hz}, 2 \mathrm{H}), 7.39(\mathrm{dd}, J=6.4 \mathrm{~Hz}, 6.4 \mathrm{~Hz}, 1 \mathrm{H}), 7.28(\mathrm{~s}, 1 \mathrm{H}), 7.13(\mathrm{dd}, J=6.4 \mathrm{~Hz}$, $6.4 \mathrm{~Hz}, 1 \mathrm{H}), 7.08-6.89(\mathrm{~m}, 6 \mathrm{H}), 6.52-6.42(\mathrm{~m}, 3 \mathrm{H}), 5.70(\mathrm{dd}, J=8.4 \mathrm{~Hz}, 2.0 \mathrm{~Hz}, 1 \mathrm{H}), 5.63(\mathrm{dd}$, $J=8.0 \mathrm{~Hz}, 2.0 \mathrm{~Hz}, 1 \mathrm{H}), 3.39$ (s, 3H). ${ }^{13} \mathrm{C} \mathrm{NMR}\left(100 \mathrm{MHz}, \mathrm{CDCl}_{3}\right) \delta(\mathrm{ppm}): 164.9,164.4$, $164.3,164.2$, 162.7 $162.6,162.5,162.4,162.3,160.2$, 160.1 160.0, 156.5, 156.0, 155.6, 154.6, $149.9,148.5,148.5,139.9,138.9,138.8,136.4,128.0,127.9,127.8,127.5,124.7,123.7,123.6$, $123.5,123.4,117.4,114.2,114.0,113.9,113.7,107.9,99.1,99.0,98.9,98.7,98.6,98.4,40.2$. MALDI-TOF-MS m/z: 849.87 [M-PF$\left._{6}^{-}\right]^{+}$.

$\left[\boldsymbol{I r}(\boldsymbol{p q l})_{2}(\boldsymbol{b m p})\right]^{+} \boldsymbol{P F}_{\mathbf{6}}(\mathbf{I r} 2)$. The mixture of cyclometalated iridium(III) chloro-bridged dimer $\left[\operatorname{Ir}(\mathrm{pql})_{2} \mathrm{Cl}\right]_{2}(200 \mathrm{mg}, 0.16 \mathrm{mmol})$ and 4-([2,2'-bipyridin]-4-yl(methyl)amino)phenol (100 mg, $0.36 \mathrm{mmol})$ was dissolved in the solution of dichloromethane/methanol $(\mathrm{v} / \mathrm{v}=2 / 1)$, being heated at $50{ }^{\circ} \mathrm{C}$ under nitrogen for $6 \mathrm{~h}$. The mixture was then cooled to room temperature and stirred for another $4 \mathrm{~h}$ with $\mathrm{KPF}_{6}(600 \mathrm{mg}, 3.26 \mathrm{mmol})$ added into the solution. Lastly, the mixture was evaporated under reduced pressure and purified by silica gel chromatograph using dichloromethane/methanol $(\mathrm{v} / \mathrm{v}=90 / 1)$ as eluent to obtain orange-red solid, complex Ir2. Yield: 55\%. ${ }^{1} \mathrm{H}$ NMR $\left(400 \mathrm{MHz}, \mathrm{CDCl}_{3}\right) \delta(\mathrm{ppm}): 8.22-8.12(\mathrm{~m}, 4 \mathrm{H}), 8.08(\mathrm{~d}, J=5.6 \mathrm{~Hz}, 1 \mathrm{H})$, $7.98(\mathrm{~d}, J=8.0 \mathrm{~Hz}, 1 \mathrm{H}), 7.93(\mathrm{~d}, J=8.0 \mathrm{~Hz}, 1 \mathrm{H}), 7.88-7.82(\mathrm{~m}, 2 \mathrm{H}), 7.69(\mathrm{dd}, J=8.0 \mathrm{~Hz}, 1.2$ $\mathrm{Hz}, 2 \mathrm{H}), 7.52-7.47$ (m, 2H), 7.40-7.25 (m, 4H), 7.15-7.03 (m, 4H), 6.95 (ddd, $J=8.8 \mathrm{~Hz}, 7.6$ $\mathrm{Hz}, 1.2 \mathrm{~Hz}, 1 \mathrm{H}), 6.89(\mathrm{~d}, J=8.4 \mathrm{~Hz}, 2 \mathrm{H}), 6.83(\mathrm{~d}, J=8.4 \mathrm{~Hz}, 2 \mathrm{H}), 6.76(\mathrm{ddd}, J=7.2 \mathrm{~Hz}, 7.2$ $\mathrm{Hz}, 0.8 \mathrm{~Hz}, 1 \mathrm{H}), 6.69$ (ddd, $J=7.2 \mathrm{~Hz}, 7.2 \mathrm{~Hz}, 0.8 \mathrm{~Hz}, 1 \mathrm{H}), 6.53$ (d, J=7.2 Hz, 1H), 6.48 (d, $J=7.6 \mathrm{~Hz}, 1 \mathrm{H}), 6.39(\mathrm{~d}, J=4.8 \mathrm{~Hz}, 1 \mathrm{H}), 3,23(\mathrm{~s}, 3 \mathrm{H}) .{ }^{13} \mathrm{C}$ NMR $\left(100 \mathrm{MHz}, \mathrm{DMSO}_{-} \mathrm{d}_{6}\right) \delta$ (ppm): 170.4, 170.3 157.0, 156.3, 155.2, 153.1, 152.0, 151.2, 149.9, 147.6, 147.3, 146.6, 146.2, $146.1,140.6,139.8,138.1,136.0,134.3,134.1,133.8,129.9,129.7,128.2,128.1,128.0,127.9$, 127.1, 125.4, 124.9, 124.8, 124.1, 123.8, 122.8, 122.7, 121.3, 118.6, 118.5, 117.1, 111.6, 106.9,

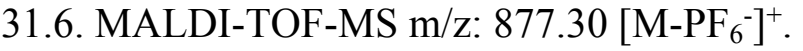

$\left[\operatorname{Ir}(\boldsymbol{t p q})_{2}(\boldsymbol{b m p})\right]^{+} \boldsymbol{P F}_{\mathbf{6}}$ (Ir3). A mixture of cyclometalated iridium(III) chloro-bridged dimer $\left[\operatorname{Ir}(\mathrm{tpq})_{2} \mathrm{Cl}\right]_{2}(200 \mathrm{mg}, 0.15 \mathrm{mmol})$ and 4-([2,2'-bipyridin]-4-yl(methyl)amino)phenol (85 mg, $0.31 \mathrm{mmol})$ in solution of dichloromethane/methanol $(\mathrm{v} / \mathrm{v}=2 / 1)$ was heated at $50{ }^{\circ} \mathrm{C}$ under nitrogen for $6 \mathrm{~h}$. The mixture was then cooled to room temperature and stirred for another $4 \mathrm{~h}$ after the addition of $\mathrm{KPF}_{6}(570 \mathrm{mg}, 3.10 \mathrm{mmol})$. Lastly, the reaction was filtered and the residue was evaporated under reduced pressure and purified by silica gel chromatograph using dichloromethane/methanol ( $\mathrm{v} / \mathrm{v}=80 / 1)$ as eluent to get the maroon solid product, complex Ir3. Yield: 47\%. ${ }^{1} \mathrm{H}$ NMR (400 MHz, DMSO-d 6 ) $\delta$ (ppm): 9.71 (s, 1H), 8.46 (s, 1H), 8.39 (dd, $J=$ $12.4 \mathrm{~Hz}, 8.8 \mathrm{~Hz}, 2 \mathrm{H}), 8.06$ (dd, $J=8.0 \mathrm{~Hz}, 8.0 \mathrm{~Hz}, 1.2 \mathrm{~Hz}, 1 \mathrm{H}), 7.99-7.87$ (m, 5H), 7.66 (ddd, $J=6.4 \mathrm{~Hz}, 6.4 \mathrm{~Hz}, 0.8 \mathrm{~Hz}, 1 \mathrm{H}), 7.62(\mathrm{~d}, J=4.4 \mathrm{~Hz}, 1 \mathrm{H}), 7.57(\mathrm{~d}, J=4.8 \mathrm{~Hz}, 1 \mathrm{H}), 7.50(\mathrm{~s}, 1 \mathrm{H})$, 7.42-7.36 (m, 2H), $7.30(\mathrm{~d}, J=6.8 \mathrm{~Hz}, 1 \mathrm{H}), 7.24(\mathrm{ddd}, J=6.8 \mathrm{~Hz}, 6.8 \mathrm{~Hz}, 1.2 \mathrm{~Hz}, 1 \mathrm{H}), 7.18(\mathrm{~d}$, $J=8.8 \mathrm{~Hz}, 1 \mathrm{H}), 7.12-7.03(\mathrm{~m}, 4 \mathrm{H}), 6.82(\mathrm{~d}, J=8.8 \mathrm{~Hz}, 2 \mathrm{H}), 6.59$ (d, $J=4.4 \mathrm{~Hz}, 1 \mathrm{H}), 6.10$ (dd, $J=12.0 \mathrm{~Hz}, 4.8 \mathrm{~Hz}, 2 \mathrm{H}), 3.32(\mathrm{~s}, 3 \mathrm{H}) .{ }^{13} \mathrm{C}$ NMR $\left(100 \mathrm{MHz}, \mathrm{DMSO}-\mathrm{d}_{6}\right) \delta(\mathrm{ppm}): 166.3,166.2$, 157.3, 157.1, 156.7, 156.1 , 155.5, 155.4, 148.4, 148.3, 148.2, 146.8, 135.9, 133.5, 133.3, 133.2, 133.0, 131.9, 131.7, 130.0, 129.9, 128.6, 128.2, 126.5, 126.4, 126.1, 124.4, 123.2, 123.1, 118.1, 118.0, 117.2, 111.8, 107.1, 40.7. MALDI-TOF-MS m/z: $889.36\left[\mathrm{M}-\mathrm{PF}_{6}^{-}\right]^{+}$.

[Ir(btq) $\left.{ }_{2}(\boldsymbol{b m p})\right]^{+} \mathrm{PF}_{6}-(\mathbf{I r} 4)$. A mixture containing cyclometalated iridium(III) chloro-bridged dimer $\left[\operatorname{Ir}(\mathrm{btq})_{2} \mathrm{Cl}\right]_{2}(200 \mathrm{mg}, 0.13 \mathrm{mmol})$ and 4-([2,2'-bipyridin]-4-yl(methyl)amino)phenol (75 $\mathrm{mg}, 0.27 \mathrm{mmol})$ in solution of dichloromethane/methanol $(\mathrm{v} / \mathrm{v}=2 / 1)$ was heated at $50{ }^{\circ} \mathrm{C}$ under nitrogen for $6 \mathrm{~h}$. The mixture was then cooled down to room temperature and stirred for another $4 \mathrm{~h}$ after adding $\mathrm{KPF}_{6}(500 \mathrm{mg}, 2.71 \mathrm{mmol})$. Lastly, the reaction was filtered, evaporated under reduced pressure and purified by silica gel chromatograph using dichloromethane/methanol 
$(\mathrm{v} / \mathrm{v}=90 / 1)$ as eluent to get reddish brown solid, complex Ir4. Yield: 45\%. ${ }^{1} \mathrm{H}$ NMR $(400 \mathrm{MHz}$, $\left.\mathrm{CDCl}_{3}\right) \delta(\mathrm{ppm}): 9.04(\mathrm{dd}, J=7.6 \mathrm{~Hz}, 7.6 \mathrm{~Hz}, 1 \mathrm{H}), 8.03(\mathrm{dd}, J=8.0 \mathrm{~Hz}, 8.0 \mathrm{~Hz}, 1 \mathrm{H}), 7.90(\mathrm{~d}$, $J=7.6 \mathrm{~Hz}, 2 \mathrm{H}), 7.83-7.74(\mathrm{~m}, 7 \mathrm{H}), 7.59(\mathrm{~d}, J=6.4 \mathrm{~Hz}, 1 \mathrm{H}), 7.38(\mathrm{~d}, J=6.8 \mathrm{~Hz}, 1 \mathrm{H}), 7.33(\mathrm{~d}$, $J=6.8 \mathrm{~Hz}, 1 \mathrm{H}), 7.27-7.22(\mathrm{~m}, 2 \mathrm{H}), 7.17-7.09(\mathrm{~m}, 3 \mathrm{H}), 6.93(\mathrm{~d}, J=8.0 \mathrm{~Hz}, 2 \mathrm{H}), 6.87(\mathrm{~d}, J=$ $8.0 \mathrm{~Hz}, 2 \mathrm{H}), 6.72-6.65(\mathrm{~m}, 2 \mathrm{H}), 6.28(\mathrm{~s}, 1 \mathrm{H}), 6.16(\mathrm{~d}, J=8.0 \mathrm{~Hz}, 1 \mathrm{H}), 6.13(\mathrm{~d}, J=8.0 \mathrm{~Hz}, 1 \mathrm{H})$, $3.36(\mathrm{~s}, 3 \mathrm{H}) .{ }^{13} \mathrm{C}$ NMR $\left(100 \mathrm{MHz}, \mathrm{CDCl}_{3}\right) \delta(\mathrm{ppm}): 165.7,165.6,157.7,156.8,156.5,155.9$, 155.5, 154.7, 150.2, 148.8, 144.7, 144.4, 143.9, 143.7, 141.6, 141.4, 140.0, 139.3, 137.1, 137.0, 136.3, 135.9, 135.3, 132.3, 132.2, 128.8, 128.0, 127.9, 127.7, 127.6, 127.1, 127.0, 126.5, 126.2, $126.1,125.1,125.0,124.8,124.5,124.4,122.3,122.2,119.7,119.6,117.4,114.1,40.3$. MALDI-TOF-MS m/z: 989.43 [M-PF6 $\left.{ }^{-}\right]^{+}$.
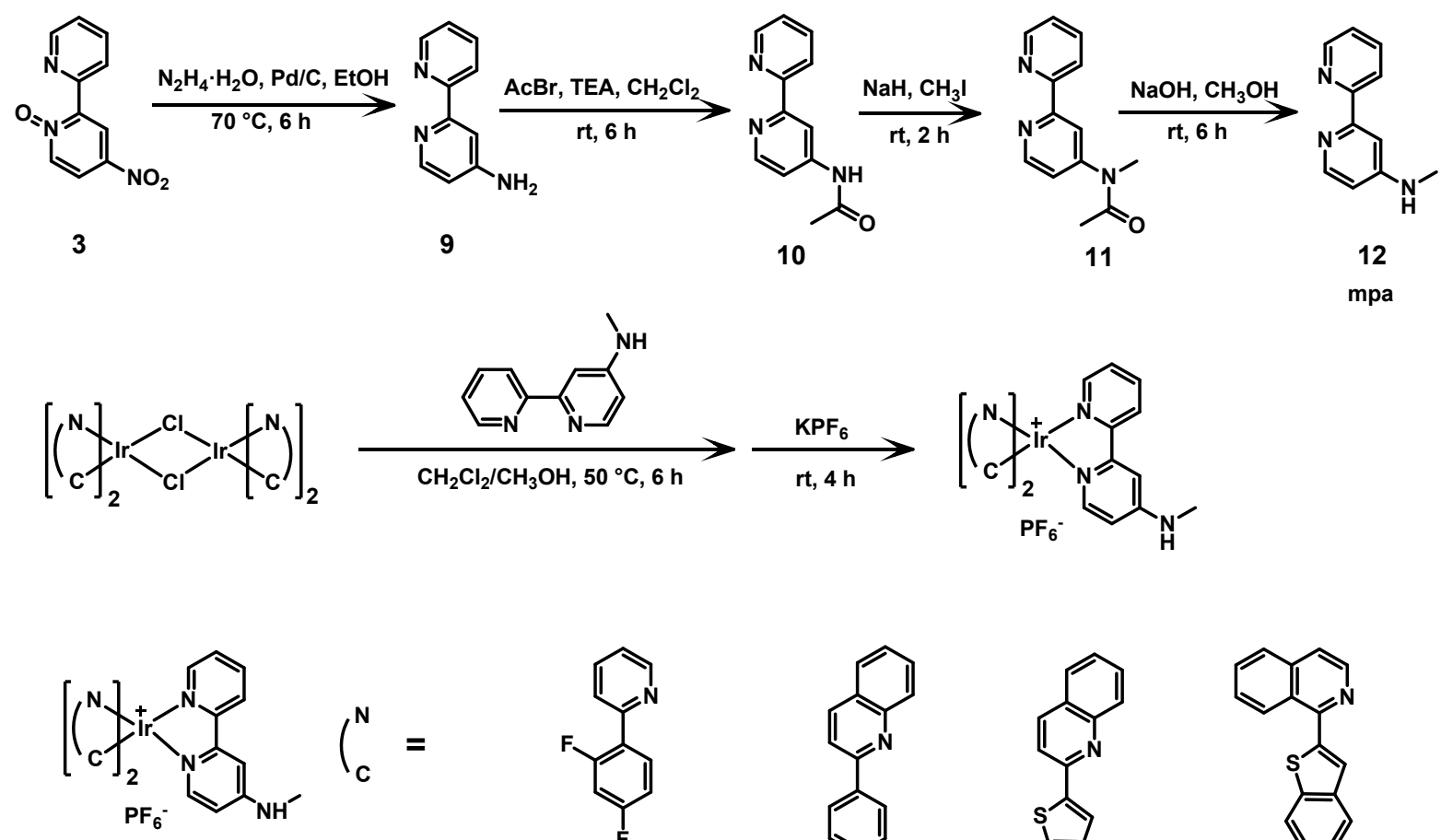

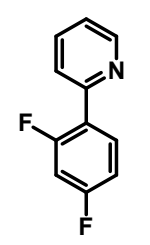

Ir1*

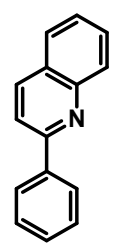

Ir2*

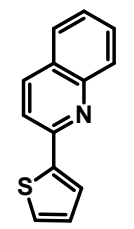

Ir3*

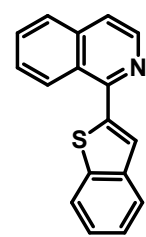

Ir4*

$\left[\operatorname{lr}(\mathrm{fpp})_{2}(\mathrm{mpa})\right]^{+} \mathrm{PF}_{6}^{-} \quad\left[\operatorname{lr}(\mathrm{pql})_{2}(\mathrm{mpa})\right]^{+} \mathrm{PF}_{6}^{-} \quad\left[\operatorname{lr}(\mathrm{tpq})_{2}(\mathrm{mpa})\right]^{+} \mathrm{PF}_{6}^{-} \quad\left[\operatorname{lr}(\mathrm{btq})_{2}(\mathrm{mpa})\right]^{+} \mathrm{PF}_{6}^{-}$

Scheme S2 Synthetic procedure of complex Ir1*, Ir2*, Ir3* and Ir4*

[2,2'-bipyridin]-4-amine (9). An oven-dried Schlenk tube was prepared for the mixture of compound $3(1.0 \mathrm{~g}, 4.6 \mathrm{mmol})$, hydrazine hydrate $(1.1 \mathrm{~mL}, 10.1 \mathrm{mmol})$ and $\mathrm{Pd} / \mathrm{C}(300.0 \mathrm{mg})$ in solution of ethanol $(50 \mathrm{~mL})$. And then it was heated in the atmosphere of the $\mathrm{N}_{2}$ at the $70{ }^{\circ} \mathrm{C}$ for $6 \mathrm{~h}$. The mixture was then cooled down to room temperature and filtered. Lastly, the residue was evaporated under reduced pressure and recrystallized from dichloromethane to give the white, needled solid. Yield: $85 \% .{ }^{1} \mathrm{H}$ NMR $\left(400 \mathrm{MHz}, \mathrm{CDCl}_{3}\right) \delta(\mathrm{ppm}): 8.63(\mathrm{~d}, J=4.4 \mathrm{~Hz}$, $1 \mathrm{H}), 8.33(\mathrm{~d}, J=8.0 \mathrm{~Hz}, 1 \mathrm{H}), 8.29(\mathrm{~d}, J=5.6 \mathrm{~Hz}, 1 \mathrm{H}), 7.80-7.75(\mathrm{~m}, 1 \mathrm{H}), 7.65(\mathrm{~d}, J=2.4 \mathrm{~Hz}$, 1H) 7.28-7.25 (m, 1H), 6.54-6.52 (m, 1H), $4.33(\mathrm{~s}, 2 \mathrm{H}) .{ }^{13} \mathrm{C} \mathrm{NMR}\left(100 \mathrm{MHz}, \mathrm{CDCl}_{3}\right) \delta(\mathrm{ppm})$ : $156.6,156.3,153.9,149.8,148.9,136.9,123.6,121.2,109.7,106.9$.

$N$-([2,2'-bipyridin]-4-yl)acetamide (10). An oven-dried Schlenk tube was prepared for the mixture of compound $9(500 \mathrm{mg}, 2.82 \mathrm{mmol})$ and triethylamine $(0.59 \mathrm{~mL}, 4.23 \mathrm{mmol}) \mathrm{a}$ in 
solution of anhydrous dichloromethane $(20 \mathrm{~mL})$. The mixture was cooled to $0{ }^{\circ} \mathrm{C}$ in an ice bath under $\mathrm{N}_{2}$. Then, acetyl bromide $(0.25 \mathrm{~mL}, 3.39 \mathrm{mmol})$ was added into the mixture slowly and the mixture was warmed to ambient temperature (by removing the ice bath) and stirred for $6 \mathrm{~h}$. $\mathrm{H}_{2} \mathrm{O}(2.0 \mathrm{~mL})$ was added rapidly to quench the reaction and the mixture was diluted with $\mathrm{CH}_{2} \mathrm{Cl}_{2}$. The biphasic mixture was then transferred to a separatory funnel and the organic layer was washed sequentially with sodium bicarbonate solution, dried over anhydrous $\mathrm{Na}_{2} \mathrm{SO}_{4}$, filtered, and concentrated under reduced pressure. The residue was purified by silica gel chromatograph using dichloromethane/methanol $(\mathrm{v} / \mathrm{v}=150 / 1)$ to give white solid. Yield: $87 \%$. ${ }^{1} \mathrm{H}$ NMR (400 MHz, DMSO-d $\left.{ }_{6}\right) \delta(\mathrm{ppm}): 10.45(\mathrm{~s}, 1 \mathrm{H}), 8.67-8.65(\mathrm{~m}, 1 \mathrm{H}), 8.58(\mathrm{~d}, J=2.4 \mathrm{~Hz}$, $1 \mathrm{H}), 8.50(\mathrm{~d}, J=5.6 \mathrm{~Hz}, 1 \mathrm{H}), 8.35(\mathrm{~d}, J=8.0 \mathrm{~Hz}, 1 \mathrm{H}), 7.91(\mathrm{dt}, J=7.6 \mathrm{~Hz}, 1.6 \mathrm{~Hz}, 1 \mathrm{H}), 7.66$ $(\mathrm{dd}, J=5.6 \mathrm{~Hz}, 2.4 \mathrm{~Hz}, 1 \mathrm{H}), 7.43(\mathrm{ddd}, J=7.6 \mathrm{~Hz}, 4.8 \mathrm{~Hz}, 1.2 \mathrm{~Hz}, 1 \mathrm{H}), 2.10(\mathrm{~s}, 3 \mathrm{H}) .{ }^{13} \mathrm{C} \mathrm{NMR}$ $\left(100 \mathrm{MHz}, \mathrm{DMSO}_{6}\right) \delta$ (ppm): 170.1, 156.6, 155.7, 150.6, 149.7, 147.3, 137.7, 124.7, 120.9, $113.7,110.2,24.7$.

$N$-([2,2'-bipyridin]-4-yl)-N-methylacetamide (11). An oven-dried Schlenk tube was prepared for the mixture of compound $10(500.0 \mathrm{mg}, 2.3 \mathrm{mmol})$, sodium hydride $(60 \%$ in oil, $110.4 \mathrm{mg}$, $3.4 \mathrm{mmol})$ and $\mathrm{N}, \mathrm{N}$-dimethylformamide $(20 \mathrm{~mL})$. After 30 minutes of stir at $0{ }^{\circ} \mathrm{C}, \mathrm{CH}_{3} \mathrm{I}(0.29$ $\mathrm{mL}, 3.45 \mathrm{mmol}$ ) was added and the mixture was stirred for $2 \mathrm{~h}$. Then, $\mathrm{H}_{2} \mathrm{O}(2.0 \mathrm{~mL})$ was added rapidly to quench the reaction and the mixture was diluted with $\mathrm{CH}_{2} \mathrm{Cl}_{2}(30 \mathrm{~mL})$. The biphasic mixture was transferred to a separatory funnel and the organic layer which was collected and dried over anhydrous $\mathrm{Na}_{2} \mathrm{SO}_{4}$, filtered, and concentrated under reduced pressure. The residue was purified by silica gel chromatograph using dichloromethane/methanol $(\mathrm{v} / \mathrm{v}=200 / 1)$ to get the product. Yield: $78 \% .{ }^{1} \mathrm{H}$ NMR $\left(400 \mathrm{MHz}, \mathrm{CDCl}_{3}\right) \delta(\mathrm{ppm}): 8.66-8.63(\mathrm{~m}, 2 \mathrm{H}), 8.38(\mathrm{~d}, J=$ $7.6 \mathrm{~Hz}, 1 \mathrm{H}), 8.26(\mathrm{~d}, J=2.0 \mathrm{~Hz}, 1 \mathrm{H}), 7.80(\mathrm{dt}, 7.6 \mathrm{~Hz}, 1.2 \mathrm{~Hz}, 1 \mathrm{H}), 7.30$ (dd, $J=7.6 \mathrm{~Hz}, 5.2$ $\mathrm{Hz}, 1 \mathrm{H}), 7.15$ (d, $J=3.6 \mathrm{~Hz}, 1 \mathrm{H}), 3.33(\mathrm{~s}, 3 \mathrm{H}), 2.04$ (s, 3H). ${ }^{13} \mathrm{C}$ NMR $\left(100 \mathrm{MHz}, \mathrm{CDCl}_{3}\right) \delta$ (ppm): 169.9, 158.0, 155.1, 152.6, 150.5, 149.2, 137.1, 124.2, 121.2, 120.9, 118.0.

N-methyl-[2,2'-bipyridin]-4-amine (12). A mixture of compound 11 (400 mg, $1.76 \mathrm{mmol}$ ) and sodium hydroxide $(700 \mathrm{mg}, 17.5 \mathrm{mmol})$ in methanol $(20 \mathrm{~mL})$ was stirred for $6 \mathrm{~h}$ at ambient temperature. Then, Saturated ammonium chloride was added to quench the reaction and the mixture was diluted with $\mathrm{CH}_{2} \mathrm{Cl}_{2}(30 \mathrm{~mL})$. The biphasic mixture was transferred to a separatory funnel and the organic layer which was collected and dried over anhydrous $\mathrm{Na}_{2} \mathrm{SO}_{4}$, filtered, and concentrated under reduced pressure. The residue was purified by silica gel chromatograph using dichloromethane/methanol $(\mathrm{v} / \mathrm{v}=130 / 1)$ to get the product. Yield: $72 \%$. ${ }^{1} \mathrm{H}$ NMR (400 $\left.\mathrm{MHz}, \mathrm{CDCl}_{3}\right) \delta(\mathrm{ppm}): 8.60(\mathrm{~d}, J=2.8 \mathrm{~Hz}, 1 \mathrm{H}), 8.31(\mathrm{~d}, J=8.0 \mathrm{~Hz}, 1 \mathrm{H}), 8.23(\mathrm{~d}, J=5.2 \mathrm{~Hz}$, $1 \mathrm{H}), 7.74(\mathrm{t}, J=7.2 \mathrm{~Hz}, 1 \mathrm{H}), 7.55(\mathrm{~s}, 1 \mathrm{H}), 7.23(\mathrm{~m}, 1 \mathrm{H}), 6.42(\mathrm{~d}, 2.8 \mathrm{~Hz}, 1 \mathrm{H}), 4.72(\mathrm{~s}, 1 \mathrm{H}), 2.85$ (s, 3H). ${ }^{13} \mathrm{C} \mathrm{NMR}\left(100 \mathrm{MHz}, \mathrm{CDCl}_{3}\right) \delta(\mathrm{ppm}): 156.5,156.1,155.4,149.3,148.9,136.9,123.5$, $121.2,107.3,104.5,29.5$.

$\left[\operatorname{Ir}(f p p)_{2}(\boldsymbol{m p a})\right]^{+} \boldsymbol{P F}_{6}-(\operatorname{Ir} 1 *)$. A mixture of cyclometalated iridium(III) chloro-bridged dimer $\left[\operatorname{Ir}(\mathrm{fpp})_{2} \mathrm{Cl}\right]_{2}(100 \mathrm{mg}, 0.08 \mathrm{mmol})$ and $\mathrm{N}$-methyl-[2,2'-bipyridin]-4-amine $(30 \mathrm{mg}, 0.16 \mathrm{mmol})$ in solution of dichloromethane/methanol $(\mathrm{v} / \mathrm{v}=2 / 1)$ was heated at $50{ }^{\circ} \mathrm{C}$ under nitrogen for 6 $\mathrm{h}$. The mixture was then cooled to room temperature and stirred for another $4 \mathrm{~h}$ after the addition of $\mathrm{KPF}_{6}$ (300 mg, $\left.1.63 \mathrm{mmol}\right)$. Being filtered and evaporated under reduced pressure. the residue was purified by silica gel chromatograph using dichloromethane/methanol $(\mathrm{v} / \mathrm{v}=80 / 1)$ to give the yellow-green solid, complex Ir1 ${ }^{*}$ Yield: $47 \% .{ }^{1} \mathrm{H}$ NMR $(400 \mathrm{MHz}$, DMSO-d 6 ) $\delta$ (ppm): 8.28-8.21 (m, 3H), 8.03-7.99 (m, 2H), $7.86(\mathrm{~d}, J=4.8 \mathrm{~Hz}, 1 \mathrm{H}), 7.77(\mathrm{~d}, J=5.6 \mathrm{~Hz}, 1 \mathrm{H})$, 7.70-7.61 (m, 3H), 6.95-6.88 (m, 2H), 6.69 (dd, $J=6.8 \mathrm{~Hz}, 2.4 \mathrm{~Hz}, 1 \mathrm{H}), 6.46(\mathrm{dd}, J=8.4 \mathrm{~Hz}$, $2.4 \mathrm{~Hz}, 1 \mathrm{H}), 5.56$ (dd, $J=8.4 \mathrm{~Hz}, 2.4 \mathrm{~Hz}, 1 \mathrm{H}), 2.90$ (s, 3H). ${ }^{13} \mathrm{C}$ NMR $\left(100 \mathrm{MHz}, \mathrm{DMSO}-\mathrm{d}_{6}\right)$ $\delta(\mathrm{ppm}): 164.7,164.6,164.5,164.4,163.7,163.6,163.4,163.3,162.6,162.5,162.4,162.2$, $162.1,162.0,161.9,160.0,159.9,159.8,156.8,156.7,156.6,156.5,156.4,156.0,155.9,140.3$, 
$140.2,129.0,128.3,128.0,124.9,123.9,123.8,123.7,123.6,113.9,113.8,113.6,113.4,99.4$, 99.3, 99.2, 99.1, 98.9, 98.8, 29.4. MALDI-TOF-MS m/z: $757.23\left[\mathrm{M}-\mathrm{PF}_{6}{ }^{-}\right]^{+}$.

$\left[\operatorname{Ir}(\mathbf{p q l})_{2}(\mathbf{m p a})\right]^{+} \mathbf{P F}_{\mathbf{6}}-(\boldsymbol{I r} 2 *)$. The mixture of cyclometalated iridium(III) chloro-bridged dimer $\left[\operatorname{Ir}(\mathrm{pql})_{2} \mathrm{Cl}\right]_{2}(100 \mathrm{mg}, 0.08 \mathrm{mmol})$ and $\mathrm{N}$-methyl-[2,2'-bipyridin]-4-amine $(30 \mathrm{mg}, 0.16 \mathrm{mmol})$ was dissolved in the solution of dichloromethane/methanol $(\mathrm{v} / \mathrm{v}=2 / 1)$, being heated at $50{ }^{\circ} \mathrm{C}$ under nitrogen for $6 \mathrm{~h}$. The mixture was then cooled to room temperature and stirred for another $4 \mathrm{~h}$ after the addition of $\mathrm{KPF}_{6}(300 \mathrm{mg}, 1.63 \mathrm{mmol})$. Ater being filtered and evaporated under reduced pressure, the residue was purified by silica gel chromatograph using dichloromethane/methanol $(\mathrm{v} / \mathrm{v}=90 / 1)$ as eluent to obtain orange-red solid, complex Ir2*. Yield: 50\%. ${ }^{1} \mathrm{H}$ NMR (400 MHz, DMSO-d $\left.{ }_{6}\right) \delta(\mathrm{ppm}): 8.53-8.48(\mathrm{~m}, 4 \mathrm{H}), 8.23(\mathrm{dd}, J=8.8 \mathrm{~Hz}$, $8.8 \mathrm{~Hz}, 2 \mathrm{H}), 8.03-7.98(\mathrm{~m}, 2 \mathrm{H}), 7.94(\mathrm{dd}, J=8.0 \mathrm{~Hz}, 0.8 \mathrm{~Hz}, 1 \mathrm{H}), 7.91(\mathrm{~d}, J=8.0 \mathrm{~Hz}, 1 \mathrm{H})$, $7.56(\mathrm{dd}, J=7.6 \mathrm{~Hz}, 7.6 \mathrm{~Hz}, 1 \mathrm{H}), 7.49-7.35(\mathrm{~m}, 5 \mathrm{H}), 6.78(\mathrm{~d}, J=7.6 \mathrm{~Hz}, 1 \mathrm{H}), 6.73(\mathrm{~d}, J=7.6$ $\mathrm{Hz}, 1 \mathrm{H}), 6.64(\mathrm{dd}, J=6.4 \mathrm{~Hz}, 2.0 \mathrm{~Hz}, 1 \mathrm{H}), 6.40(\mathrm{~d}, J=7.6 \mathrm{~Hz}, 1 \mathrm{H}), 6.34(\mathrm{~d}, J=7.6 \mathrm{~Hz}, 1 \mathrm{H})$, $2.73(\mathrm{~d}, J=4.4 \mathrm{~Hz}, 3 \mathrm{H}) .{ }^{13} \mathrm{C}$ NMR $\left(100 \mathrm{MHz}, \mathrm{DMSO}_{-} \mathrm{d}_{6}\right) \delta(\mathrm{ppm}): 170.5,170.2,156.7,156.6$, 156.0, 153.2, 152.2, 147.5, 147.4, 146.6, 146.3, 140.6, 140.5, 139.8, 134.4, 134.1, 131.3, 131.2, $130.9,130.8,129.8,129.7,128.1,127.9,127.8,127.1,127.0,125.1,124.8,122.8,122.7,118.5$,

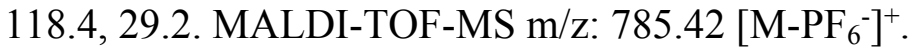

$\left[\operatorname{Ir}(\boldsymbol{t p q})_{2}(\boldsymbol{m p a})\right]^{+} \boldsymbol{P F}_{6}-(\operatorname{Ir} 3 *)$. A mixture of cyclometalated iridium(III) chloro-bridged dimer $\left[\operatorname{Ir}(\mathrm{tpq})_{2} \mathrm{Cl}\right]_{2}(100 \mathrm{mg}, 0.08 \mathrm{mmol})$ and $\mathrm{N}$-methyl-[2,2'-bipyridin]-4-amine $(30 \mathrm{mg}, 0.16 \mathrm{mmol})$ in solution of dichloromethane/methanol $(\mathrm{v} / \mathrm{v}=2 / 1)$ was heated at $50{ }^{\circ} \mathrm{C}$ under nitrogen for 6 h. After the addition of $\mathrm{KPF}_{6}(300 \mathrm{mg}, 1.63 \mathrm{mmol})$ for another $4 \mathrm{~h}$, the mixture was filtered and evaporated under reduced pressure. Lastly, the reaction was purified by silica gel chromatograph using dichloromethane/methanol $(\mathrm{v} / \mathrm{v}=80 / 1)$ as eluent to get the maroon solid product, complex Ir3*. Yield: 45\%. ${ }^{1} \mathrm{H}$ NMR (400 MHz, DMSO-d $\left.{ }_{6}\right) \delta(\mathrm{ppm}): 8.38(\mathrm{dd}, J=8.4$ $\mathrm{Hz}, 8.4 \mathrm{~Hz}, 2 \mathrm{H}), 8.06$ (ddd, $J=8.0 \mathrm{~Hz}, 8.0 \mathrm{~Hz}, 1.2 \mathrm{~Hz}, 1 \mathrm{H}), 7.97$ (d, $J=8.8 \mathrm{~Hz}, 1.2 \mathrm{~Hz}, 2 \mathrm{H})$, $7.93(\mathrm{~d}, J=5.6 \mathrm{~Hz}, 1 \mathrm{H}), 7.88(\mathrm{dd}, J=8.4 \mathrm{~Hz}, 8.4 \mathrm{~Hz}, 2 \mathrm{H}), 7.65-7.63(\mathrm{~m}, 1 \mathrm{H}), 7.62(\mathrm{~d}, J=4.8$ $\mathrm{Hz}, 1 \mathrm{H}), 7.59$ (d, $J=4.8 \mathrm{~Hz}, 1 \mathrm{H}), 7.50(\mathrm{~s}, 1 \mathrm{H}), 7.17$ (ddd, $J=8.8 \mathrm{~Hz}, 6.8 \mathrm{~Hz}, 1.6 \mathrm{~Hz}, 1 \mathrm{H}), 7.10-$ $7.03(\mathrm{~m}, 2 \mathrm{H}), 6.70(\mathrm{dd}, J=6.4 \mathrm{~Hz}, 2.4 \mathrm{~Hz}, 1 \mathrm{H}), 6.14(\mathrm{~d}, J=4.8 \mathrm{~Hz}, 1 \mathrm{H}), 6.08(\mathrm{dd}, J=4.8 \mathrm{~Hz}$, $1 \mathrm{H}), 2.78(\mathrm{~d}, J=4.4 \mathrm{~Hz}, 1 \mathrm{H}) .{ }^{13} \mathrm{C}$ NMR $\left(100 \mathrm{MHz}, \mathrm{DMSO}-\mathrm{d}_{6}\right) \delta(\mathrm{ppm}): 166.3,166.1,157.0$, $156.3,156.1,148.3,148.2$, 148.1 141.1, 141.0, 140.9, 140.3, 140.1, 133.5, 133.4, 133.3, 133.2, 133.1, 133.0, 131.7, 131.6, 130.1, 130.0, 129.9, 128.5, 126.4, 126.3, 126.1, 126.0, 123.3, 123.2, 123.1, 118.1, 118.0, 29.2. MALDI-TOF-MS m/z: 797.44 [M-PF $\left.{ }_{6}^{-}\right]^{+}$.

$\left[\boldsymbol{I r}(\boldsymbol{b t q})_{2}(\boldsymbol{m p a})\right]^{+} \boldsymbol{P F}_{\mathbf{6}}-\left(\boldsymbol{I r} 4^{*}\right)$. A mixture of cyclometalated iridium(III) chloro-bridged dimer $\left[\operatorname{Ir}(\mathrm{btq})_{2} \mathrm{Cl}\right]_{2}(100 \mathrm{mg}, 0.07 \mathrm{mmol})$ and $\mathrm{N}$-methyl-[2,2'-bipyridin]-4-amine $(25 \mathrm{mg}, 0.14 \mathrm{mmol})$ was added into the solution of dichloromethane/methanol $(\mathrm{v} / \mathrm{v}=2 / 1)$, being heated under nitrogen at $50{ }^{\circ} \mathrm{C}$ for $6 \mathrm{~h}$. The mixture was then cooled down to room temperature and stirred for $4 \mathrm{~h}$ after adding $\mathrm{KPF}_{6}(250 \mathrm{mg}, 1.36 \mathrm{mmol})$. After being filtered and evaporated under reduced pressure, the residue was purified by silica gel chromatograph using dichloromethane/methanol (v/v $=70 / 1)$ to get reddish brown solid, complex Ir4*. Yield: 47\%. ${ }^{1} \mathrm{H} \mathrm{NMR}\left(400 \mathrm{MHz}, \mathrm{CDCl}_{3}\right) \delta(\mathrm{ppm}): 9.07(\mathrm{~d}, J=8.0 \mathrm{~Hz}, 2 \mathrm{H}), 8.49(\mathrm{~d}, J=7.6 \mathrm{~Hz}, 1 \mathrm{H}), 7.99$ $(\mathrm{d}, J=7.6 \mathrm{~Hz}, 1 \mathrm{H}), 7.37-7.78(\mathrm{~m}, 10 \mathrm{H}), 7.58(\mathrm{~d}, J=6.0 \mathrm{~Hz}, 1 \mathrm{H}), 7.36(\mathrm{~d}, J=6.4 \mathrm{~Hz}, 1 \mathrm{H}), 7.28-$ $7.22(\mathrm{~m}, 5 \mathrm{H}), 7.20-7.15(\mathrm{~m}, 2 \mathrm{H}), 6.74(\mathrm{~d}, J=7.2 \mathrm{~Hz}, 1 \mathrm{H}), 6.70(\mathrm{~d}, J=7.8 \mathrm{~Hz}, 1 \mathrm{H}), 6.29(\mathrm{~d}, J$ $=5.6 \mathrm{~Hz}, 1 \mathrm{H}), 6.20(\mathrm{~d}, J=8.0 \mathrm{~Hz}, 1 \mathrm{H}), 6.14(\mathrm{~d}, J=8.0 \mathrm{~Hz}, 1 \mathrm{H}), 2.84(\mathrm{~s}, 3 \mathrm{H}) .{ }^{13} \mathrm{C}$ NMR $(100$ $\left.\mathrm{MHz}, \mathrm{CDCl}_{3}\right) \delta(\mathrm{ppm}): 165.9,165.7,158.2,157.0,156.9,156.2,150.1,144.7,144.5,144.0$, $143.8,141.5,141.2,139.7,137.0,136.9,135.9,135.4,132.3,132.3,128.8,127.6,127.5,127.1$, $126.6,126.5,126.3,125.1,125.0,124.5,124.4,122.3,122.2$, 119.5, 119.4, 77.2, 29.7. MALDI-

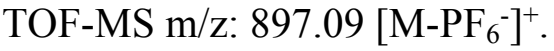




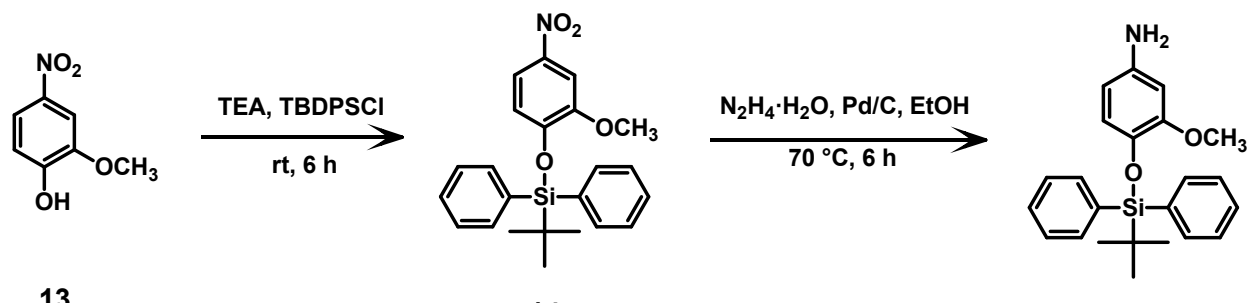

13

14

15
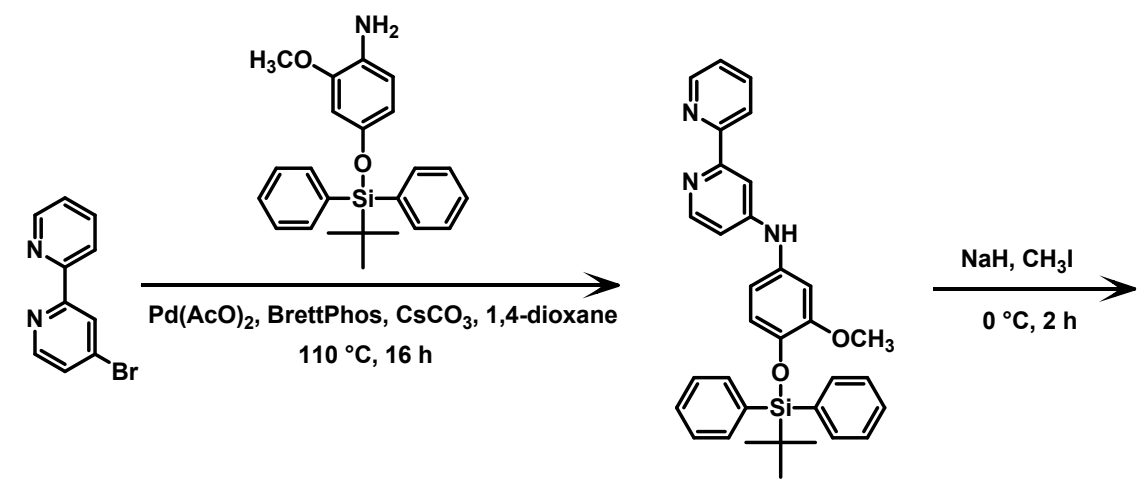

16

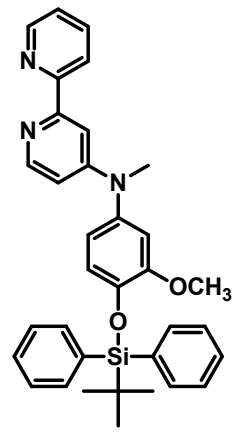

17
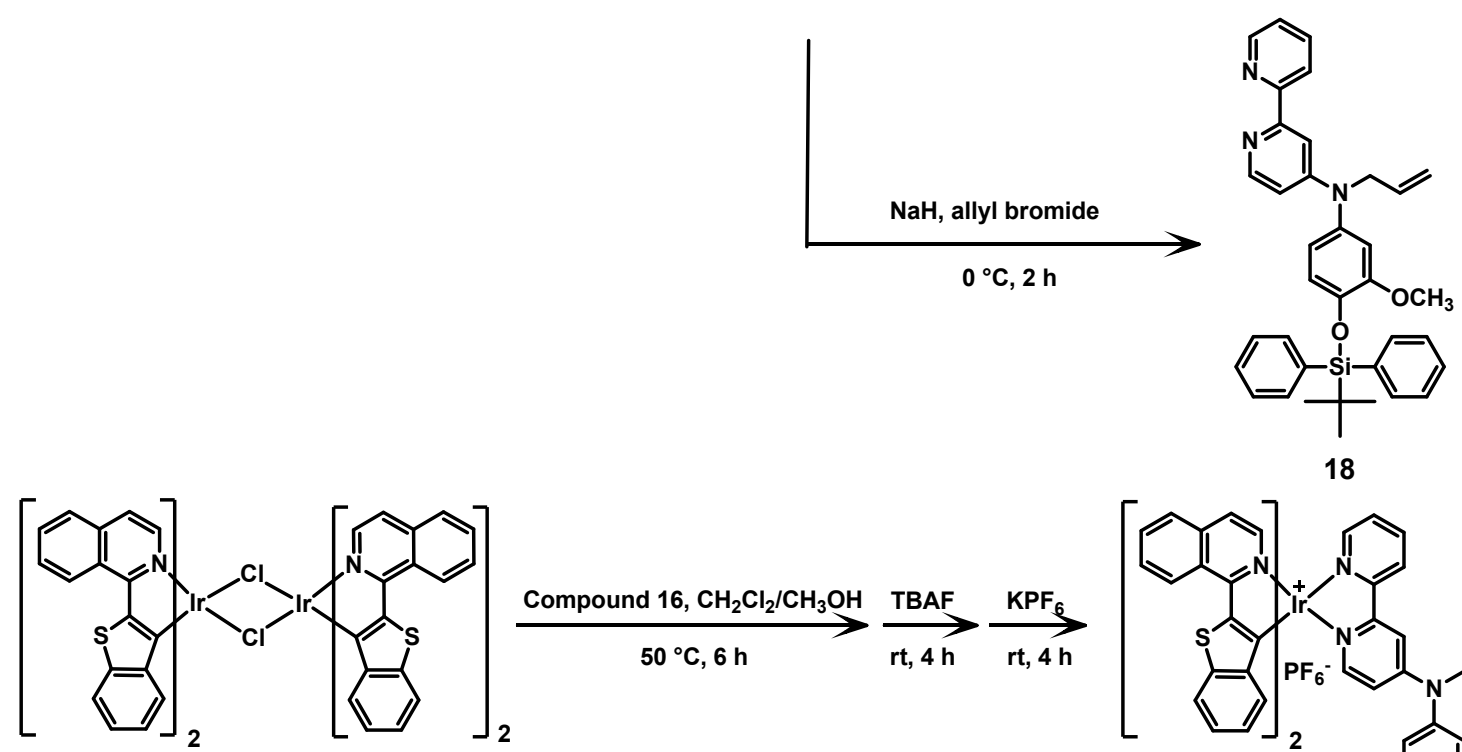

$\underset{50^{\circ} \mathrm{C}, 6 \mathrm{~h}}{\stackrel{\text { Compound } 16, \mathrm{CH}_{2} \mathrm{Cl}_{2} / \mathrm{CH}_{3} \mathrm{OH}}{\longrightarrow}} \underset{\mathrm{rt}, 4 \mathrm{~h}}{\stackrel{\mathrm{TBAF}}{\longrightarrow}} \underset{\mathrm{rt}, 4 \mathrm{~h}}{\stackrel{\mathrm{KPF}_{6}}{\longrightarrow}}$

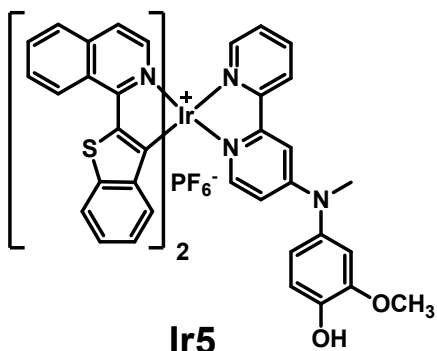

$\left[\operatorname{lr}(\mathrm{btq})_{2}(\mathrm{pmp})\right]^{+} \mathrm{PF}_{6}^{-}$
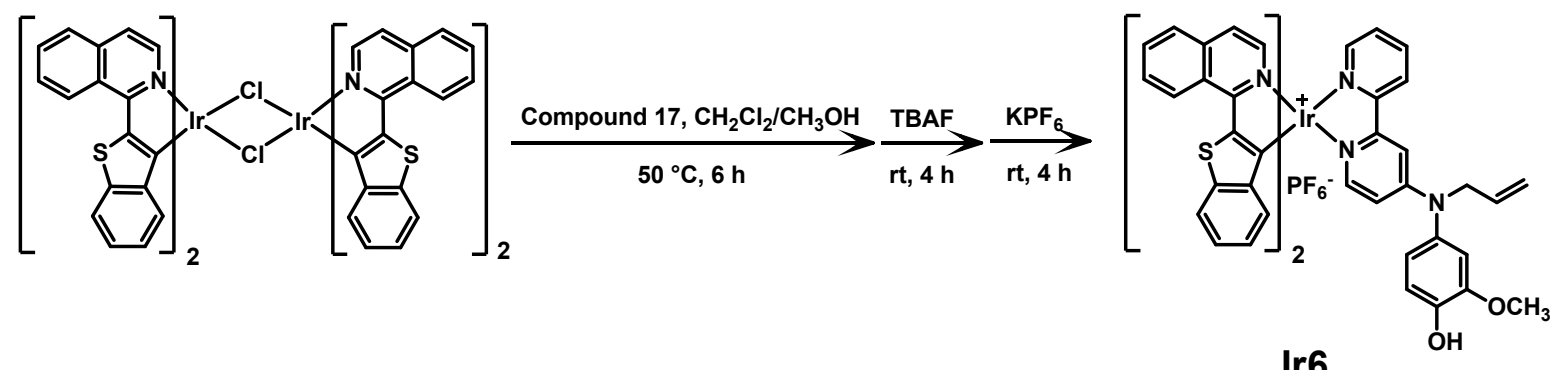

Ir6

$\left[\operatorname{lr}(\mathbf{b t q})_{2}(\mathrm{pap})\right]^{+} \mathrm{PF}_{6}^{-}$

Scheme S3 Synthetic procedure of complex Ir5, Ir6

Tert-butyl(2-methoxy-4-nitrophenoxy)diphenylsilane (14). A mixture of 2-methoxy-4nitrophenol (1.0 g, $5.9 \mathrm{mmol})$, TBDPSCl $(2.3 \mathrm{~mL}, 8.8 \mathrm{mmol})$ and TEA $(0.98 \mathrm{~mL}, 7.1 \mathrm{mmol})$ in 
solution of $\mathrm{CH}_{3} \mathrm{CN}$ (30 mL) was added to the oven-dried Schlenk tube. And then, the mixture was stirred at the room temperature for $6 \mathrm{~h}$. After that, it was evaporated under reduced pressure and purified by silica gel chromatograph using dichloromethane/petroleum ether $(\mathrm{v} / \mathrm{v}=1 / 30)$ to give the canary yellow solid. Yield: $85 \% .{ }^{1} \mathrm{H}$ NMR $\left(400 \mathrm{MHz}, \mathrm{CDCl}_{3}\right): 7.78(\mathrm{dd}, J=8.0 \mathrm{~Hz}$, $2.4 \mathrm{~Hz}, 4 \mathrm{H}), 7.72(\mathrm{~d}, J=2.8 \mathrm{~Hz}, 1 \mathrm{H}), 7.66(\mathrm{dd}, J=8.8 \mathrm{~Hz}, 2.8 \mathrm{~Hz}, 1 \mathrm{H}), 7.47-7.40(\mathrm{~m}, 6 \mathrm{H})$, $6.80(\mathrm{~d}, J=8.8 \mathrm{~Hz}, 1 \mathrm{H}), 3.68(\mathrm{~s}, 3 \mathrm{H}), 1.22(\mathrm{~s}, 9 \mathrm{H}) . \delta(\mathrm{ppm}): .{ }^{13} \mathrm{C} \mathrm{NMR}\left(100 \mathrm{MHz}, \mathrm{CDCl}_{3}\right) \delta$ (ppm): 151.3, 150.6, 142.1, 135.3, 132.4, 130.2, 127.9, 119.5, 117.3, 107.3, 55.6, 26.6, 19.9.

4-((tert-butyldiphenylsilyl)oxy)-3-methoxyaniline (15). An oven-dried Schlenk tube was prepared for the mixture of compound $14(800 \mathrm{mg}, 1.6 \mathrm{mmol})$, hydrazine hydrate $(230 \mu \mathrm{L}, 4.8$ $\mathrm{mmol})$ and $\mathrm{Pd} / \mathrm{C}(300.0 \mathrm{mg})$ in solution of ethanol $(30 \mathrm{~mL})$. And then it was heated in the atmosphere of the $\mathrm{N}_{2}$ at the $70{ }^{\circ} \mathrm{C}$ for $6 \mathrm{~h}$. The mixture was then cooled down to room temperature and filtered. The residue was evaporated under reduced pressure and purified by silica gel chromatograph using ethyl acetate/petroleum ether $(\mathrm{v} / \mathrm{v}=1 / 10)$ to give the brown product. Yield: $80 \% .{ }^{1} \mathrm{H}$ NMR $\left(400 \mathrm{MHz}, \mathrm{CDCl}_{3}\right) \delta(\mathrm{ppm}):$ 7.75-7.72 (m, 4H), 7.42-7.33 (m, $6 \mathrm{H}), 6.54(\mathrm{~d}, J=8.8 \mathrm{~Hz}, 1 \mathrm{H}), 6.18(\mathrm{~d}, J=2.8 \mathrm{~Hz}, 1 \mathrm{H}), 6.00(\mathrm{dd}, J=8.0 \mathrm{~Hz}, 2.8 \mathrm{~Hz}, 1 \mathrm{H}), 3.54$ $(\mathrm{s}, 3 \mathrm{H}), 1.12(\mathrm{~s}, 9 \mathrm{H})$.

N-(4-((tert-butyldiphenylsilyl)oxy)-3-methoxyphenyl)-[2,2'-bipyridin]-4-amine (16). An oven-dried Schlenk tube was charged with 4-bromo-2,2'-bipyridine (500 mg, $2.13 \mathrm{mmol}$ ), 4methoxyaniline (980 mg, $2.56 \mathrm{mmol}), \mathrm{Pd}(\mathrm{AcO})_{2}(47 \mathrm{mg}, 0.21 \mathrm{mmol})$, dicyclohexyl[3,6dimethoxy-2',4',6'-tris(1-methylethyl)[1,1'-biphenyl]-2-yl]phosphine (BrettPhos) (118 mg, $0.22 \mathrm{mmol})$ and $\mathrm{Cs}_{2} \mathrm{CO}_{3}(1.72 \mathrm{~g}, 5.32 \mathrm{mmol})$, and flushed with $\mathrm{N}_{2}$ gas for $30 \mathrm{~min}$. Then anhydrous 1,4-dioxane $(60 \mathrm{~mL})$ was introduced. The resulting mixture was first stirred under $\mathrm{N}_{2}$ at room temperature for $30 \mathrm{~min}$ and then at $100{ }^{\circ} \mathrm{C}$ for $20 \mathrm{~h}$. The reaction mixture was allowed to cool to room temperature, diluted with $\mathrm{CH}_{2} \mathrm{Cl}_{2}$ and filtered. The filter cake was washed with $\mathrm{CH}_{2} \mathrm{Cl}_{2}$. The filtrate was then concentrated and the residue was purified by silica gel chromatograph using dichloromethane/methanol $(\mathrm{v} / \mathrm{v}=75 / 1)$ to give dark brown solid. Yield: $26 \%$. ${ }^{1} \mathrm{H}$ NMR $\left(400 \mathrm{MHz}, \mathrm{CDCl}_{3}\right) \delta(\mathrm{ppm}): 8.61(\mathrm{~d}, J=4.0 \mathrm{~Hz}, 1 \mathrm{H}), 8.32(\mathrm{~d}, J=8.0 \mathrm{~Hz}$, $1 \mathrm{H}), 8.28(\mathrm{~d}, J=6.0 \mathrm{~Hz}, 1 \mathrm{H}), 7.80(\mathrm{~d}, J=2.4 \mathrm{~Hz}, 1 \mathrm{H}), 7.77-7.72(\mathrm{~m}, 5 \mathrm{H}), 7.43-7.34(\mathrm{~m}, 6 \mathrm{H})$, 7.24 (ddd, $J=7.6 \mathrm{~Hz}, 4.8 \mathrm{~Hz}, 1.2 \mathrm{~Hz}, 1 \mathrm{H}), 6.71(\mathrm{~d}, J=8.4 \mathrm{~Hz}, 1 \mathrm{H}), 6.65(\mathrm{dd}, J=5.6 \mathrm{~Hz}, 2.4$ $\mathrm{Hz}, 1 \mathrm{H}), 6.61(\mathrm{~d}, J=2.4 \mathrm{~Hz}, 1 \mathrm{H}), 6.51(\mathrm{dd}, J=2.8 \mathrm{~Hz}, 1 \mathrm{H}), 6.33(\mathrm{~s}, 1 \mathrm{H}), 3.51(\mathrm{~s} .3 \mathrm{H}), 1.15$ (s, 9H) $.{ }^{13} \mathrm{C} \mathrm{NMR}\left(100 \mathrm{MHz}, \mathrm{CDCl}_{3}\right) \delta(\mathrm{ppm}): 156.8,156.4,152.7,151.1,149.9,149.0,142.3$, $136.9,135.5,133.5,133.3,129.7,127.6,123.6,121.2$, 120.6, 115.4, 108.4, 108.3, 106.8, 55.4, $26.7,19.8$.

\section{N-(4-((tert-butyldiphenylsilyl)oxy)-3-methoxyphenyl)-N-methyl-[2,2'-bipyridin]-4-amine}

(17). Compound 16 (300 mg, $0.56 \mathrm{mmol})$ was dissolved in dry DMF (15.0 mL). The solution was cooled to $0{ }^{\circ} \mathrm{C}$ in an ice bath and $\mathrm{NaH}(60 \%$ in oil, $67.2 \mathrm{mg}, 1.68 \mathrm{mmol})$ was added in one portion. After 30 minutes of stir at $0{ }^{\circ} \mathrm{C}, \mathrm{CH}_{3} \mathrm{I}(104 \mu \mathrm{L}, 1.68 \mathrm{mmol})$ was added. Immediately following addition, the mixture was warmed to ambient temperature slowly and stirred for 1.5 h. Then, $\mathrm{H}_{2} \mathrm{O}(2.0 \mathrm{~mL})$ was added rapidly to quench the reaction and the mixture was diluted with $\mathrm{CH}_{2} \mathrm{Cl}_{2}(20 \mathrm{~mL})$. The biphasic mixture was then transferred to a separatory funnel and the organic layer was washed sequentially with $\mathrm{H}_{2} \mathrm{O}$ and brine, dried over anhydrous $\mathrm{Na}_{2} \mathrm{SO}_{4}$, filtered, and concentrated under reduced pressure. The residue was purified by silica gel chromatograph using dichloromethane/methanol $(\mathrm{v} / \mathrm{v}=100 / 1)$ to give the product. Yield: $70 \%$. ${ }^{1} \mathrm{H}$ NMR $\left(400 \mathrm{MHz}, \mathrm{CDCl}_{3}\right) \delta(\mathrm{ppm}): 8.64(\mathrm{~d}, J=4.0 \mathrm{~Hz}, 1 \mathrm{H}), 8.33(\mathrm{~d}, J=8.0 \mathrm{~Hz}, 1 \mathrm{H}), 8.21$ $(\mathrm{d}, J=6.0 \mathrm{~Hz}, 1 \mathrm{H}), 7.79-7.69(\mathrm{~m}, 6 \mathrm{H}), 7.44-7.35(\mathrm{~m}, 6 \mathrm{H}), 7.26$ (ddd, $J=7.6 \mathrm{~Hz}, 4.8 \mathrm{~Hz}, 0.8$ $\mathrm{Hz}, 1 \mathrm{H}), 6.78(\mathrm{~d}, J=8.4 \mathrm{~Hz}, 1 \mathrm{H}), 6.60(\mathrm{~d}, J=2.4 \mathrm{~Hz}, 1 \mathrm{H}), 6.53(\mathrm{dd}, J=8.4 \mathrm{~Hz}, 2.8 \mathrm{~Hz}, 1 \mathrm{H})$, 6.37 (dd, $J=5.6 \mathrm{~Hz}, 2.4 \mathrm{~Hz}, 1 \mathrm{H}), 3.51$ (s, 3H), 3.33 (s, 3H), $1.16(\mathrm{~s}, 9 \mathrm{H}) .{ }^{13} \mathrm{C}$ NMR $(100 \mathrm{MHz}$, $\left.\mathrm{CDCl}_{3}\right) \delta(\mathrm{ppm}): 156.9,{ }^{\prime} 156.4,155.2,151.5,149.0,148.9,143.8,140.0,136.7,135.5,133.4$, 129.8, 127.6, 123.4, 121.3, 121.1, 119.01, 111.14, 108.5, 104.8, 55.4, 29.80, 26.8, 19.8 . 
N-allyl-N-(4-((tert-butyldiphenylsilyl)oxy)-3-methoxyphenyl)-[2,2'-bipyridin]-4-amine (18). Compound 16 (300 mg, $0.56 \mathrm{mmol})$ was dissolved in dry DMF $(15.0 \mathrm{~mL})$. The solution was cooled to $0{ }^{\circ} \mathrm{C}$ in an ice bath and $\mathrm{NaH}(60 \%$ in oil, $67.2 \mathrm{mg}, 1.68 \mathrm{mmol})$ was added in one portion. After 30 minutes of stir at $0{ }^{\circ} \mathrm{C}$, allyl bromide $(124 \mu \mathrm{L}, 1.44 \mathrm{mmol})$ was added. And then, the mixture was warmed to ambient temperature slowly and stirred for $1.5 \mathrm{~h} . \mathrm{H}_{2} \mathrm{O}(2.0$ $\mathrm{mL})$ was added rapidly to quench the reaction and the mixture was diluted with $\mathrm{CH}_{2} \mathrm{Cl}_{2}(20$ $\mathrm{mL}$ ). The biphasic mixture was then transferred to a separatory funnel and the organic layer was washed sequentially with $\mathrm{H}_{2} \mathrm{O}$ and brine, dried over anhydrous $\mathrm{Na}_{2} \mathrm{SO}_{4}$, filtered, and concentrated under reduced pressure. The residue was purified by silica gel chromatograph using dichloromethane/methanol $(\mathrm{v} / \mathrm{v}=90 / 1)$ to give the product. Yield: $60 \%$. ${ }^{1} \mathrm{H}$ NMR (400 $\left.\mathrm{MHz}, \mathrm{CDCl}_{3}\right) \delta(\mathrm{ppm}): 8.63(\mathrm{ddd}, J=4.8 \mathrm{~Hz}, 1.6 \mathrm{~Hz}, 0.8 \mathrm{~Hz}, 1 \mathrm{H}), 8.32(\mathrm{~d}, J=8.0 \mathrm{~Hz}, 1 \mathrm{H})$, $8.24(\mathrm{~d}, J=6.0 \mathrm{~Hz}, 1 \mathrm{H}), 7.77-7.72(\mathrm{~m}, 5 \mathrm{H}), 7.70(\mathrm{~d}, J=2.8 \mathrm{~Hz}, 1 \mathrm{H}), 7.44-7.35(\mathrm{~m}, 6 \mathrm{H}), 7.21$ (ddd, $J=7.6 \mathrm{~Hz}, 4.8 \mathrm{~Hz}, 1.2 \mathrm{~Hz}, 1 \mathrm{H}), 6.81(\mathrm{~d}, J=8.4 \mathrm{~Hz}, 1 \mathrm{H}), 6.63(\mathrm{~d}, J=2.4 \mathrm{~Hz}, 1 \mathrm{H}), 6.08$ $(\mathrm{dd}, J=8.4 \mathrm{~Hz}, 2.4 \mathrm{~Hz}, 1 \mathrm{H}), 6.39$ (dd, $J=6.0 \mathrm{~Hz}, 2.8 \mathrm{~Hz}, 1 \mathrm{H}), 5.97-5.87$ (m, 1H), $5.24(\mathrm{dd}, J$ $=17.2 \mathrm{~Hz}, 1.2 \mathrm{~Hz}, 1 \mathrm{H}), 5.19(\mathrm{dd}, J=10.4 \mathrm{~Hz}, 1.2 \mathrm{~Hz}, 1 \mathrm{H}), 4.31(\mathrm{~d}, J=4.8 \mathrm{~Hz}, 2 \mathrm{H}), 3.50(\mathrm{~s}$, $3 \mathrm{H}), 1.18(\mathrm{~s}, 9 \mathrm{H}) .{ }^{13} \mathrm{C} \mathrm{NMR}\left(100 \mathrm{MHz}, \mathrm{CDCl}_{3}\right) \delta(\mathrm{ppm}): 156.9,156.5,154.5,151.5,149.2$, 149.0, 143.9, 138.9, 136.7, 135.5, 133.4, 132.8, 129.8, 127.6, 123.3, 121.2, 121.1, 119.6, 117.2, $111.7,108.7,105.3,55.4,54.6,26.7,19.8$.

$\left[\boldsymbol{I r}(\boldsymbol{b t q})_{2}(\mathbf{p m p})\right]^{+} \boldsymbol{P F}_{\mathbf{6}}$ (Ir5). A mixture of cyclometalated iridium(III) chloro-bridged dimer $\left[\operatorname{Ir}(\mathrm{btq})_{2} \mathrm{Cl}\right]_{2}(200 \mathrm{mg}, 0.13 \mathrm{mmol})$ and compound $17(150 \mathrm{mg}, 0.28 \mathrm{mmol})$ in solution of dichloromethane/methanol $(\mathrm{v} / \mathrm{v}=2 / 1)$ was heated under nitrogen at $50{ }^{\circ} \mathrm{C}$ for $6 \mathrm{~h}$. The mixture was then cooled down to room temperature and stirred for $4 \mathrm{~h}$ after adding TBAF ( $450 \mathrm{mg}, 1.43$ mmol). After that, $\mathrm{KPF}_{6}(2600 \mathrm{mg}, 14.13 \mathrm{mmol})$ was added into the mixture, which was stirred for another $4 \mathrm{~h}$. Lastly, the reaction was filterd, evaporated under reduced pressure and purified by silica gel chromatograph using dichloromethane/methanol $(\mathrm{v} / \mathrm{v}=80 / 1)$ to get reddish brown solid, Yield: 50\%. ${ }^{1} \mathrm{H}$ NMR (400 MHz, $\left.\mathrm{CDCl}_{3}\right) \delta(\mathrm{ppm}): 9.05(\mathrm{dd}, \mathrm{J}=8.4 \mathrm{~Hz}, 4.4 \mathrm{~Hz}, 2 \mathrm{H}), 8.06$ $(\mathrm{dd}, J=7.6 \mathrm{~Hz}, 7.6 \mathrm{~Hz}, 1 \mathrm{H}), 7.92(\mathrm{~d}, J=7.6 \mathrm{~Hz}, 2 \mathrm{H}), 7.83-7.76(\mathrm{~m}, 7 \mathrm{H}), 7.72(\mathrm{~d}, J=6.8 \mathrm{~Hz}$, $1 \mathrm{H}), 7.63(\mathrm{~d}, J=6.8 \mathrm{~Hz}, 1 \mathrm{H}), 7.42-7.37(\mathrm{~m}, 3 \mathrm{H}), 7.18-7.11(\mathrm{~m}, 4 \mathrm{H}), 6.88(\mathrm{~d}, J=8.4 \mathrm{~Hz}, 1 \mathrm{H})$, $6.81(\mathrm{~d}, J=8.4 \mathrm{~Hz}, 1 \mathrm{H}), 6.77(\mathrm{~s}, 1 \mathrm{H}), 6.71(\mathrm{~d}, J=8.4 \mathrm{~Hz}, 1 \mathrm{H}), 6.67(\mathrm{~d}, J=8.4 \mathrm{~Hz}, 1 \mathrm{H}), 6.62$ $(\mathrm{dd}, J=7.6 \mathrm{~Hz}, 1.2 \mathrm{~Hz}, 1 \mathrm{H}), 6.28(\mathrm{~s}, 1 \mathrm{H}), 6.16(\mathrm{~d}, J=8.4 \mathrm{~Hz}, 1 \mathrm{H}), 6.13(\mathrm{~d}, J=8.4 \mathrm{~Hz}, 1 \mathrm{H})$, $3.85(\mathrm{~s}, 3 \mathrm{H}), 3.47(\mathrm{~s}, 3 \mathrm{H}) .{ }^{13} \mathrm{C} \mathrm{NMR}\left(100 \mathrm{MHz}, \mathrm{CDCl}_{3}\right) \delta(\mathrm{ppm}): 167.7,165.7,165.6,157.4$, 157.0, 156.7, 155.7, 150.2, 148.6, 147.9, 145.4, 144.7, 144.4, 143.9, 143.7, 141.8, 141.5, 139.9, $137.1,137.0,136.6,136.0,135.2,132.4,132.2,130.9,129.2,128.9,128.7,127.9,127.7,127.6$, $127.1,126.9,126.5,126.4,126.2,125.1,125.0,124.9,124.4,122.3,122.2,119.9,119.5,119.4$,

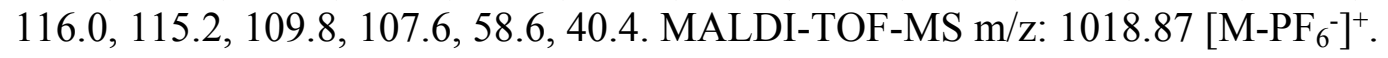

$\left[\boldsymbol{I r}(\boldsymbol{b t q})_{2}(\mathbf{p a p})\right]^{+} \boldsymbol{P F}_{\mathbf{6}}$ (Ir6). A mixture of cyclometalated iridium(III) chloro-bridged dimer $\left[\operatorname{Ir}(\mathrm{btq})_{2} \mathrm{Cl}\right]_{2}(200 \mathrm{mg}, 0.13 \mathrm{mmol})$, compound $18(150 \mathrm{mg}, 0.26 \mathrm{mmol})$ in solution of dichloromethane/methanol $(\mathrm{v} / \mathrm{v}=2 / 1)$ was heated under nitrogen at reflux for $6 \mathrm{~h}$. The mixture was then cooled down to room temperature and stirred for $4 \mathrm{~h}$ after adding TBAF (420 mg, 1.33 $\mathrm{mmol})$, which is followed by the append of $\mathrm{KPF}_{6}(2500 \mathrm{mg}, 13.5 \mathrm{mmol})$ for another $4 \mathrm{~h}$. The mixture was then cooled down to room temperature and evaporated under reduced pressure. Lastly, the reaction was purified by silica gel chromatograph using dichloromethane/methanol $(\mathrm{v} / \mathrm{v}=80 / 1)$ to get orange-red solid. Yield: $55 \% .{ }^{1} \mathrm{H}$ NMR $\left(400 \mathrm{MHz}, \mathrm{CDCl}_{3}\right) \delta(\mathrm{ppm}): 9.05(\mathrm{~d}$, $J=7.6 \mathrm{~Hz}, 2 \mathrm{H}), 8.03(\mathrm{dd}, J=7.2 \mathrm{~Hz}, 7.2 \mathrm{~Hz}, 1 \mathrm{H}), 7.94-7.91(\mathrm{~m}, 2 \mathrm{H}), 7.85-7.75(\mathrm{~m}, 8 \mathrm{H}), 7.64-$ $7.62(\mathrm{~m}, 1 \mathrm{H}), 7.42(\mathrm{~d}, J=6.4 \mathrm{~Hz}, 1 \mathrm{H}), 7.28-7.24(\mathrm{~m}, 4 \mathrm{H}), 7.17(\mathrm{~d}, J=8.0 \mathrm{~Hz}, 1 \mathrm{H}), 7.12(\mathrm{~d}, J$ $=8.0 \mathrm{~Hz}, 1 \mathrm{H}), 6.89(\mathrm{~d}, J=4.8 \mathrm{~Hz}, 1 \mathrm{H}), 6.82(\mathrm{~s}, 1 \mathrm{H}), 6.72-6.64(\mathrm{~m}, 3 \mathrm{H}), 6.32(\mathrm{~s}, 1 \mathrm{H}), 6.13(\mathrm{dd}$, $J=3.2 \mathrm{~Hz}, 8.0 \mathrm{~Hz}, 2 \mathrm{H}), 5.96-5.87(\mathrm{~m}, 1 \mathrm{H}), 5.30(\mathrm{~d}, J=4.0 \mathrm{~Hz}, 1 \mathrm{H}), 5.24(\mathrm{~d}, J=10.8 \mathrm{~Hz}, 1 \mathrm{H})$, $4.41(\mathrm{t}, J=15.6 \mathrm{~Hz}, 2 \mathrm{H}), 3.86(\mathrm{~s}, 3 \mathrm{H}) .{ }^{13} \mathrm{C} \mathrm{NMR}\left(100 \mathrm{MHz}, \mathrm{CDCl}_{3}\right) \delta(\mathrm{ppm}): 165.7,165.5$, 157.3, 156.9, 156.6, 155.4, 154.7, 150.3, 149.0, 148.0, 145.6, 144.7, 144.4, 143.9, 143.7, 141.8, 
141.6, 139.9, 137.2, 137.0, 136.0, 135.5, 135.3, 132.2, 131.5, 128.7, 128.6, 128.0, 127.8, 127.6, $127.1,127.0,126.9,126.5,126.4,126.2,126.1,125.1,125.0,124.6,124.5,124.4,122.3,122.2$, $120.2,120.1,120.0,119.6,118.0,115.2,110.3,108.0,56.4,55.5$. MALDI-TOF-MS m/z: $1045.16\left[\mathrm{M}-\mathrm{PF}_{6}^{-}\right]^{+}$.

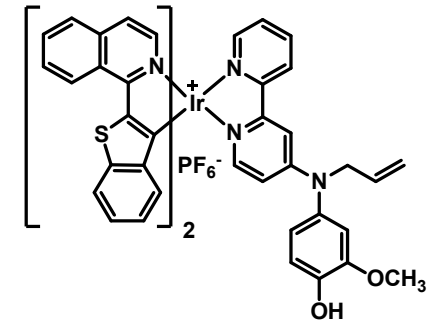

Ir 6

$\left[\operatorname{lr}(\mathrm{btq})_{2}(\mathrm{pap})\right]^{+} \mathrm{PF}_{6}{ }^{-}$

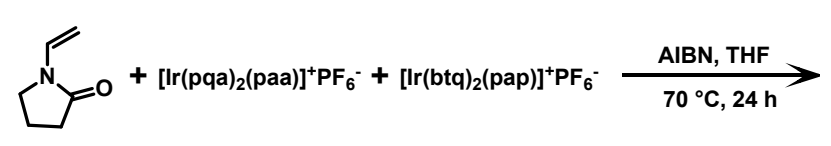

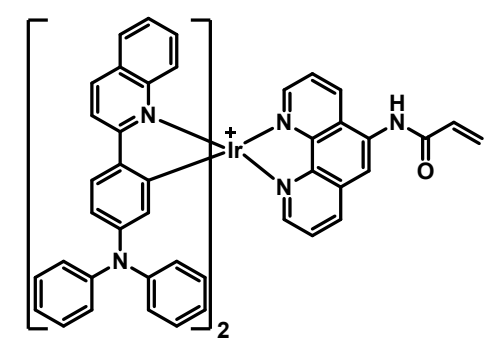

Ir7

$\left[\operatorname{Ir}(\text { pqa })_{2}(\text { paa) }]^{+} \mathrm{PF}_{6}{ }^{-}\right.$

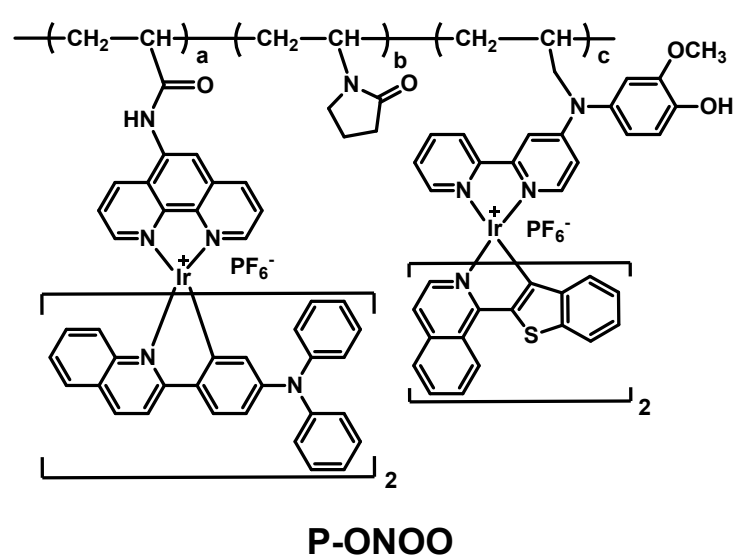

Scheme S4 Synthetic procedure of P-ONOO

$\left[\operatorname{Ir}(\boldsymbol{p q a})_{2}(\boldsymbol{p a a})\right]^{+} \boldsymbol{P F}_{\mathbf{6}}(\operatorname{Ir} 7)$. Complex $\operatorname{Ir} 7$ was synthesized and purified according to our previous report. ${ }^{4}$

Preparation of P-ONOO. A mixture of $N$-vinypyrrolidone (1000 mg, $9.01 \mathrm{mmol}$ ), 2,2azobisisobutyronitrile (4 mg, $24 \mu \mathrm{mol})$, complex Ir6 (40 mg, $33.6 \mu \mathrm{mol})$ and complex Ir7 (60 $\mathrm{mg}, 45.1 \mu \mathrm{mol})$ was dissolved in anhydrous tetrahydrofuran $(3 \mathrm{~mL})$. The solution was bubbled with dry nitrogen for 2 hours to remove dissolved oxygen and then stirred under nitrogen at $70^{\circ} \mathrm{C}$ for $24 \mathrm{~h}$. After cooling, the reaction mixture was poured into diethyl ether $(250 \mathrm{ml})$, and the obtained P-ONOO was purified by reprecipitation using dichloromethane /diethyl ether (5 $\mathrm{ml} / 250 \mathrm{ml}$ ) and dialysis. Yield: $35.3 \%$. GPC (THF, polystyrene standard): $\mathrm{M}_{\mathrm{n}}=10500, \mathrm{PDI}$ $=1.87$.

The proportions of the iridium(III) complexes units in the copolymers were determined from their absorbance in dichloromethane with the model iridium(III) complexes. The contents of the $N$-vinypyrrolidone units in the copolymers were determined from related calculations. 


\section{Supplementary Tables and Figures}

Table S1. Photophysical data for complexes and polymer at $298 \mathrm{~K}$.

\begin{tabular}{|c|c|c|c|c|}
\hline & Medium & $\lambda_{\mathrm{em}}(\mathrm{nm})$ & $\Phi_{\mathrm{PL}}$ & $\tau(\mu \mathrm{s})$ \\
\hline \multirow[t]{2}{*}{ Ir1 } & $\mathrm{CH}_{2} \mathrm{Cl}_{2}$ & - & - & - \\
\hline & $\mathrm{PBS}^{[\mathrm{a}]}$ & - & - & - \\
\hline \multirow[t]{2}{*}{ Ir2 } & $\mathrm{CH}_{2} \mathrm{Cl}_{2}$ & 559 & 0.35 & 0.66 \\
\hline & PBS[a] & 558 & 0.19 & 0.52 \\
\hline \multirow[t]{2}{*}{ Ir3 } & $\mathrm{CH}_{2} \mathrm{Cl}_{2}$ & 597 & 0.17 & 0.72 \\
\hline & $\mathrm{PBS}^{[\mathrm{a}]}$ & 597 & 0.13 & 0.43 \\
\hline \multirow[t]{2}{*}{ Ir 4} & $\mathrm{CH}_{2} \mathrm{Cl}_{2}$ & 680 & 0.04 & 0.87 \\
\hline & $\mathrm{PBS}^{[\mathrm{a}]}$ & 679 & 0.03 & 0.64 \\
\hline \multirow[t]{2}{*}{$\operatorname{Ir} 1 *$} & $\mathrm{CH}_{2} \mathrm{Cl}_{2}$ & 516 & 0.48 & 0.60 \\
\hline & $\mathrm{PBS}^{[\mathrm{a}]}$ & 517 & 0.39 & 0.21 \\
\hline \multirow[t]{2}{*}{ Ir2* } & $\mathrm{CH}_{2} \mathrm{Cl}_{2}$ & 560 & 0.51 & 0.96 \\
\hline & $\mathrm{PBS}^{[\mathrm{a}]}$ & 561 & 0.42 & 0.73 \\
\hline \multirow[t]{2}{*}{ Ir3* } & $\mathrm{CH}_{2} \mathrm{Cl}_{2}$ & 596 & 0.33 & 0.97 \\
\hline & $\mathrm{PBS}^{[\mathrm{a}]}$ & 595 & 0.27 & 0.88 \\
\hline \multirow[t]{2}{*}{ Ir 4* } & $\mathrm{CH}_{2} \mathrm{Cl}_{2}$ & 680 & 0.13 & 1.03 \\
\hline & $\mathrm{PBS}^{[\mathrm{a}]}$ & 680,743 & 0.10 & 0.98 \\
\hline \multirow[t]{2}{*}{ Ir5 } & $\mathrm{CH}_{2} \mathrm{Cl}_{2}$ & 680 & 0.03 & 0.95 \\
\hline & $\mathrm{PBS}^{[\mathrm{a}]}$ & 679,743 & 0.02 & 0.60 \\
\hline \multirow[t]{2}{*}{ Ir6 } & $\mathrm{CH}_{2} \mathrm{Cl}_{2}$ & 680 & 0.03 & 0.89 \\
\hline & $\mathrm{PBS}^{[\mathrm{a}]}$ & 680,743 & 0.02 & 0.66 \\
\hline \multirow[t]{2}{*}{ Ir7 } & $\mathrm{CH}_{2} \mathrm{Cl}_{2}$ & 602 & 0.15 & 2.18 \\
\hline & $\mathrm{PBS}^{[\mathrm{a}]}$ & 604 & 0.10 & 0.64 \\
\hline P-ONOO & $\mathrm{H}_{2} \mathrm{O}$ & $605,678 / 743$ & 0.04 & $0.60 / 0.68$ \\
\hline
\end{tabular}

[a] Containing $10 \% \mathrm{CH}_{3} \mathrm{CN}$. 
Table S2. Quantum efficiencies of iridium(III) complex probes in different viscous solution at $298 \mathrm{~K}$.

\begin{tabular}{ccccc}
\hline Complexes & Methanol & $\begin{array}{c}\text { Methanol/Glycerol } \\
(\mathrm{v} / \mathrm{v}=19 / 1)\end{array}$ & $\begin{array}{c}\text { Methanol/Glycerol } \\
(\mathrm{v} / \mathrm{v}=9 / 1)\end{array}$ & Solid \\
$\mathbf{I r 1}$ & - & - & - & 0.01 \\
$\mathbf{I r} 2$ & 0.21 & 0.22 & 0.23 & 0.04 \\
$\mathbf{I r 3}$ & 0.15 & 0.16 & 0.16 & 0.04 \\
\hline
\end{tabular}


Table S3. Calculated energy levels of the lowest triplet state for $\mathrm{N}^{\wedge} \mathrm{N}$ ligands with the TDDFT method.

Triplet-state Energy $\left(\mathrm{T}_{1}\right)^{\mathrm{a}}$


Table S4. Calculated important molecular orbits of complexes Ir1, Ir1*, Ir2, Ir2*, Ir3, Ir3*, Ir4, Ir4*, Ir5 and Ir6 with the TDDFT method.

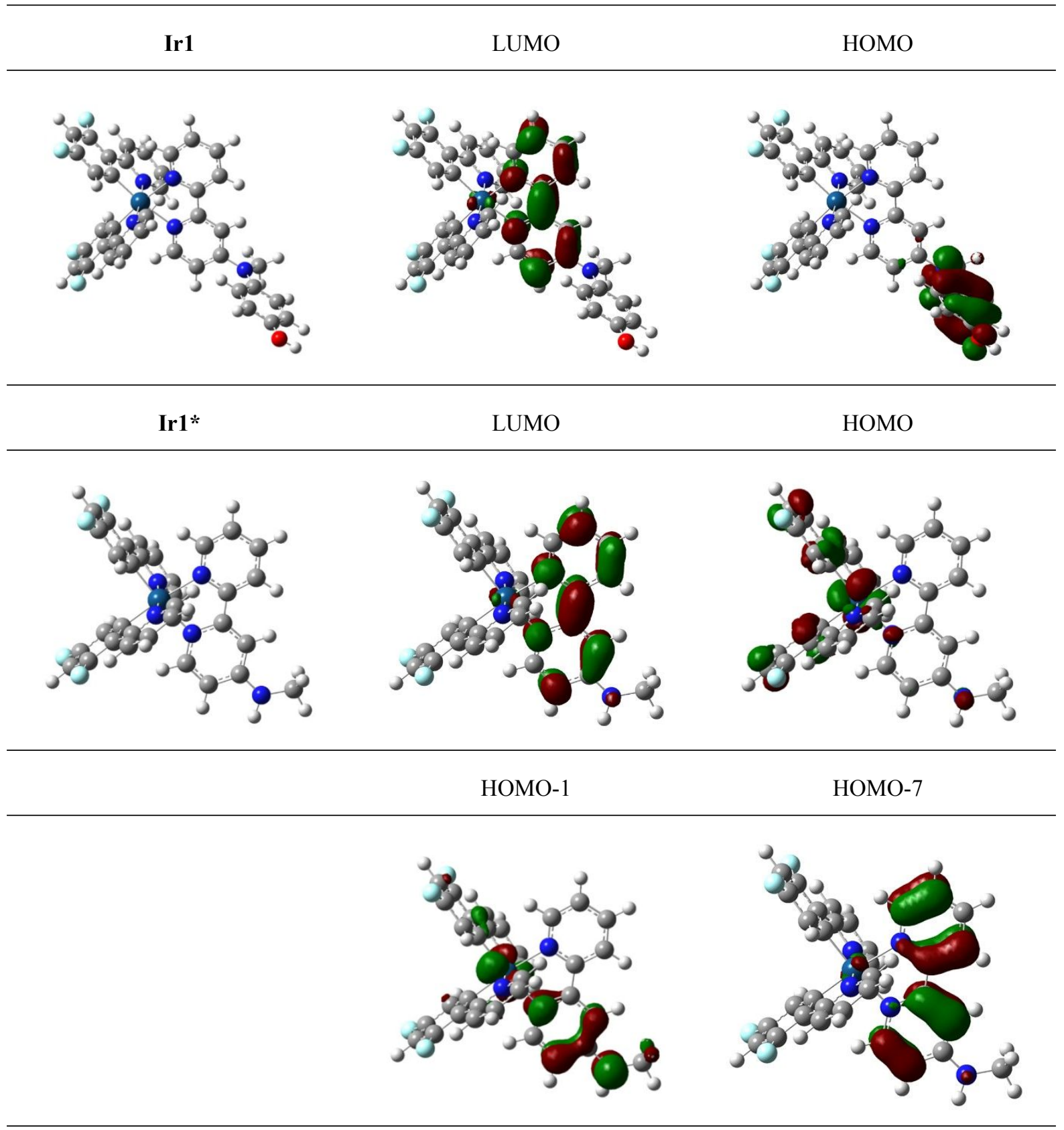




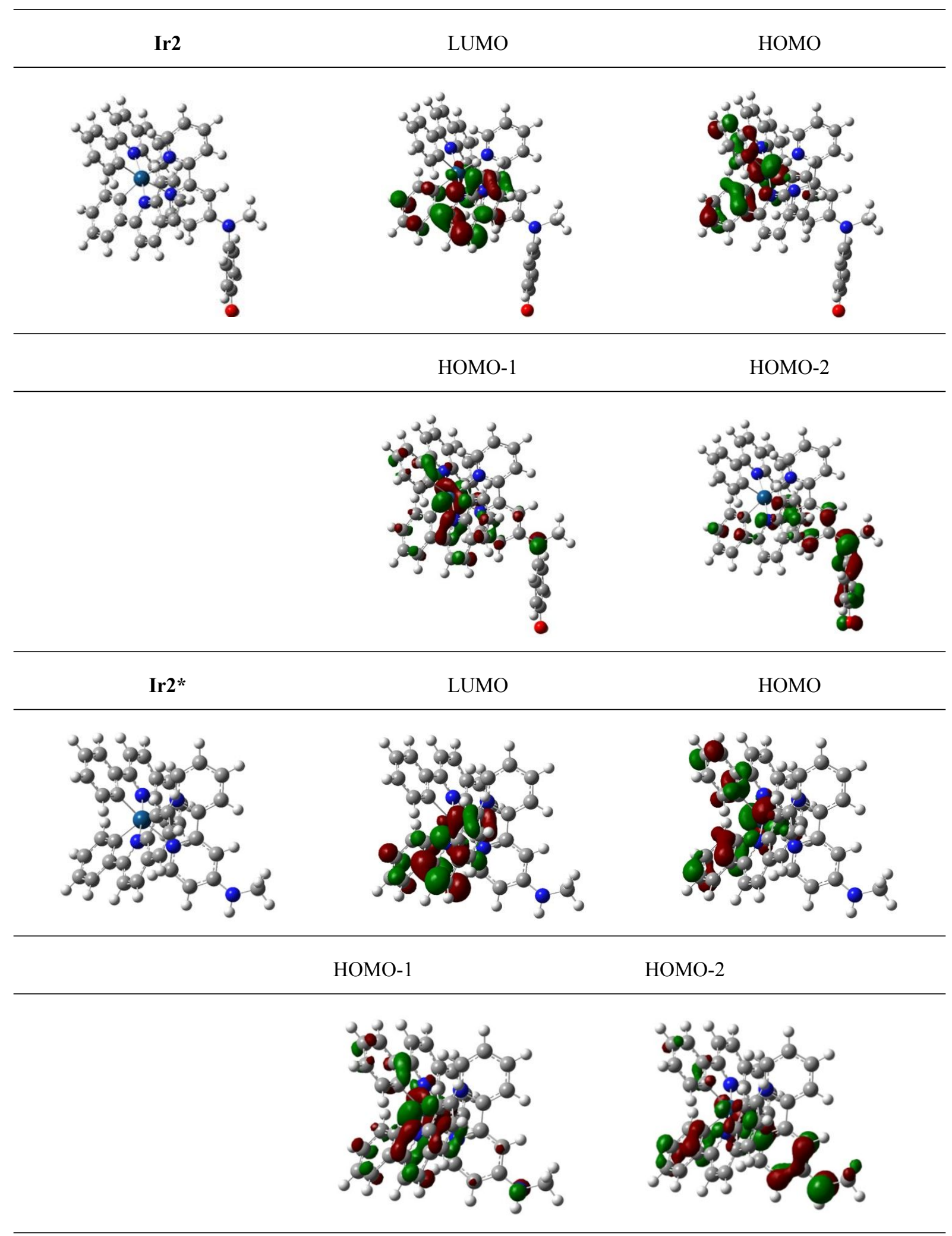




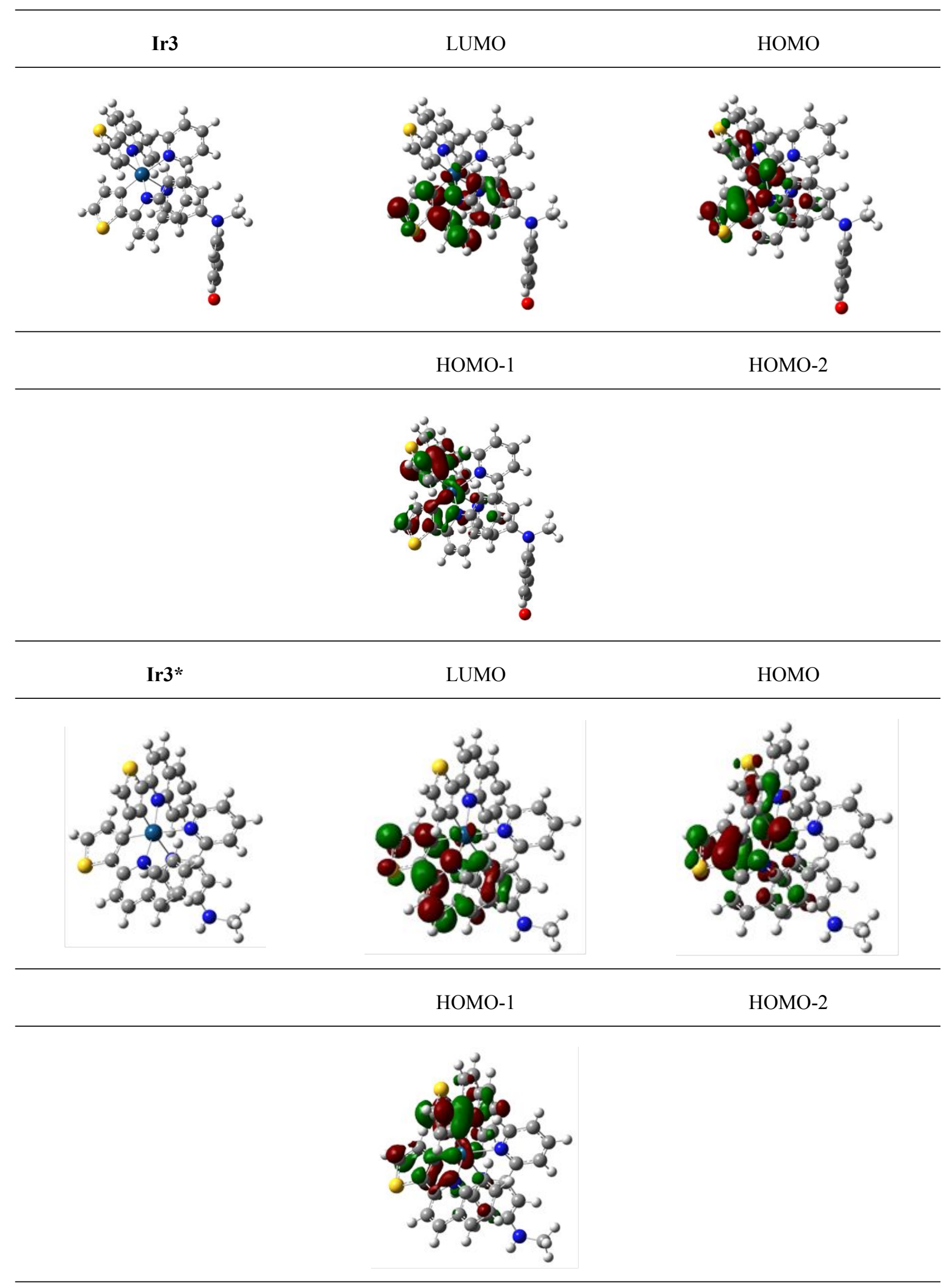




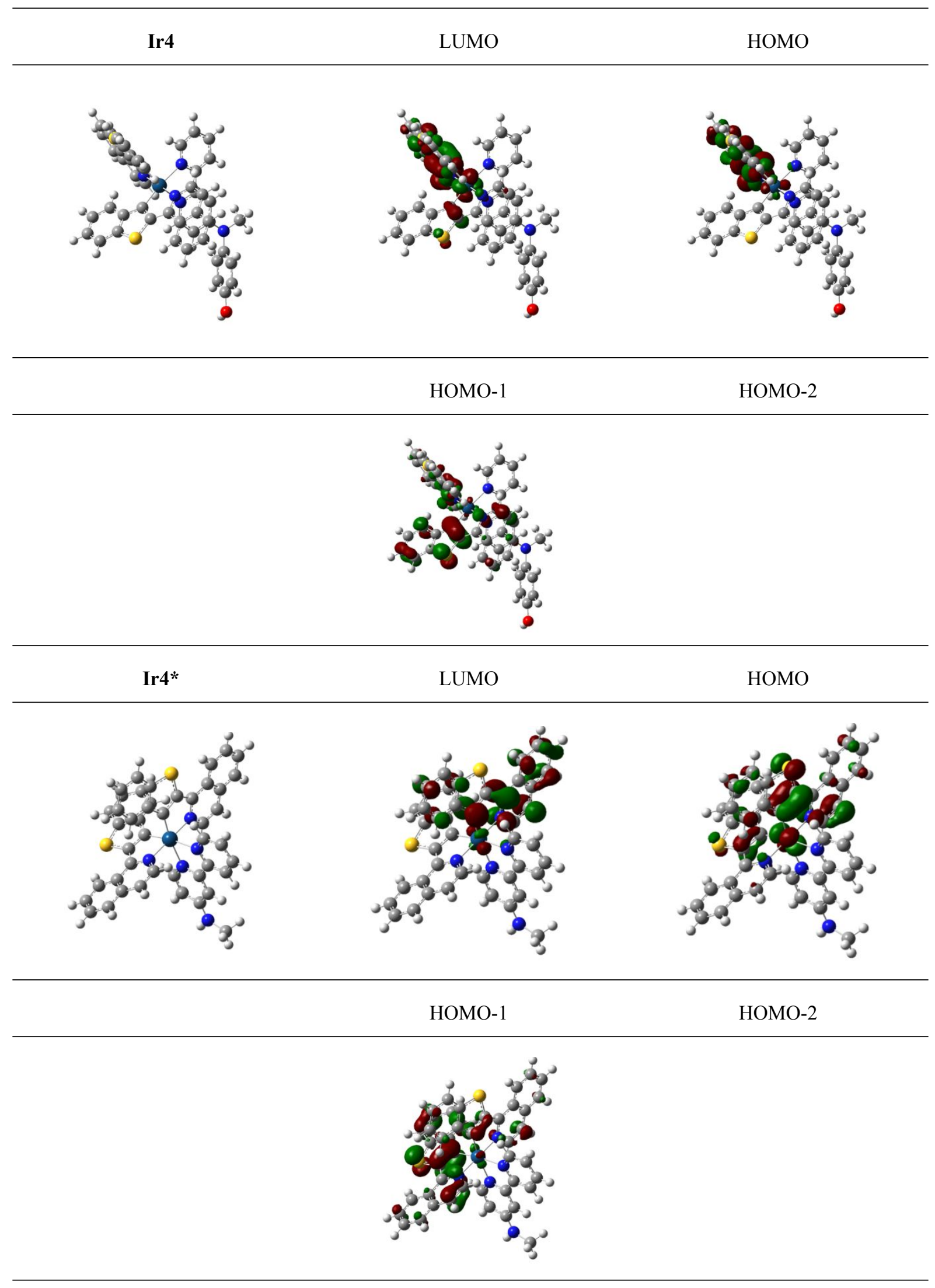




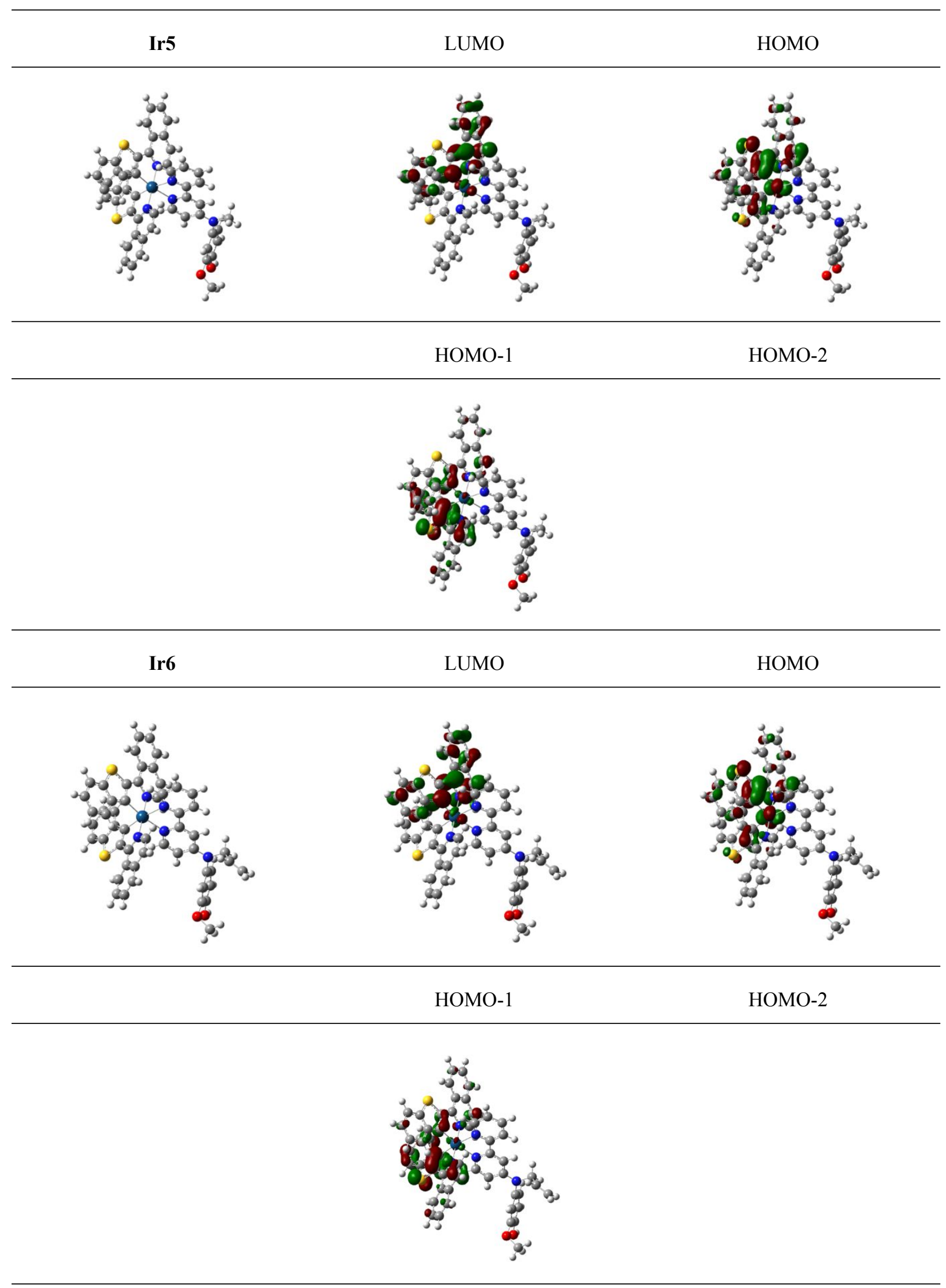


Table S5. Calculated phosphorescent emission of complexes with the TDDFT method.

\begin{tabular}{|c|c|c|c|c|}
\hline Complexes & State & $\lambda / \mathrm{nm}$ & Configuration & Character \\
\hline Ir1 & $\mathrm{T}_{1}$ & 1163 & $\mathrm{HOMO} \rightarrow \mathrm{LUMO}(99 \%)$ & ILCT \\
\hline \multirow[t]{3}{*}{$\operatorname{Ir} 1^{*}$} & $\mathrm{~T}_{1}$ & 578 & $\mathrm{HOMO} \rightarrow \mathrm{LUMO}(34 \%)$ & MLCT / LLCT \\
\hline & & & HOMO- $1 \rightarrow$ LUMO (57\%) & MLCT / LLCT \\
\hline & & & HOMO-7 $\rightarrow$ LUMO $(2 \%)$ & MLCT / ILCT \\
\hline \multirow[t]{3}{*}{ Ir2 } & $\mathrm{T}_{1}$ & 670 & $\mathrm{HOMO} \rightarrow \mathrm{LUMO}(49 \%)$ & MLCT / LLCT \\
\hline & & & HOMO-1 $\rightarrow$ LUMO (30\%) & MLCT / LLCT \\
\hline & & & HOMO-2 $\rightarrow$ LUMO (12\%) & LLCT \\
\hline \multirow[t]{3}{*}{ Ir 2* } & $\mathrm{T}_{1}$ & 681 & $\mathrm{HOMO} \rightarrow \mathrm{LUMO}(49 \%)$ & MLCT / LLCT \\
\hline & & & HOMO-1 $\rightarrow$ LUMO (35\%) & MLCT / LLCT \\
\hline & & & HOMO-2 $\rightarrow$ LUMO (6\%) & LLCT \\
\hline \multirow[t]{2}{*}{ Ir3 } & $\mathrm{T}_{1}$ & 727 & $\mathrm{HOMO} \rightarrow \mathrm{LUMO}(80 \%)$ & MLCT / LLCT / ILCT \\
\hline & & & HOMO-1 $\rightarrow$ LUMO (14\%) & MLCT / LLCT \\
\hline \multirow[t]{2}{*}{ Ir3* } & $\mathrm{T}_{1}$ & 728 & $\mathrm{HOMO} \rightarrow \mathrm{LUMO}(78 \%)$ & MLCT / LLCT / ILCT \\
\hline & & & HOMO-1 $\rightarrow$ LUMO (15\%) & MLCT / LLCT \\
\hline \multirow[t]{2}{*}{$\operatorname{Ir} 4$} & $\mathrm{~T}_{1}$ & 916 & $\mathrm{HOMO} \rightarrow \mathrm{LUMO}(84 \%)$ & MLCT / LLCT / ILCT \\
\hline & & & HOMO- $\rightarrow$ LUMO (12\%) & LLCT / ILCT \\
\hline \multirow[t]{2}{*}{ Ir4* } & $\mathrm{T}_{1}$ & 917 & $\mathrm{HOMO} \rightarrow \mathrm{LUMO}(87 \%)$ & MLCT / LLCT / ILCT \\
\hline & & & HOMO-1 $\rightarrow$ LUMO (12\%) & LLCT / ILCT \\
\hline \multirow[t]{2}{*}{ Ir5 } & $\mathrm{T}_{1}$ & 917 & $\mathrm{HOMO} \rightarrow \mathrm{LUMO}(84 \%)$ & MLCT / LLCT / ILCT \\
\hline & & & HOMO- $\rightarrow$ LUMO (12\%) & LLCT / ILCT \\
\hline \multirow[t]{2}{*}{ Ir6 } & $\mathrm{T}_{1}$ & 917 & $\mathrm{HOMO} \rightarrow \mathrm{LUMO}(83 \%)$ & MLCT / LLCT / ILCT \\
\hline & & & HOMO- $1 \rightarrow$ LUMO $(12 \%)$ & LLCT / ILCT \\
\hline
\end{tabular}



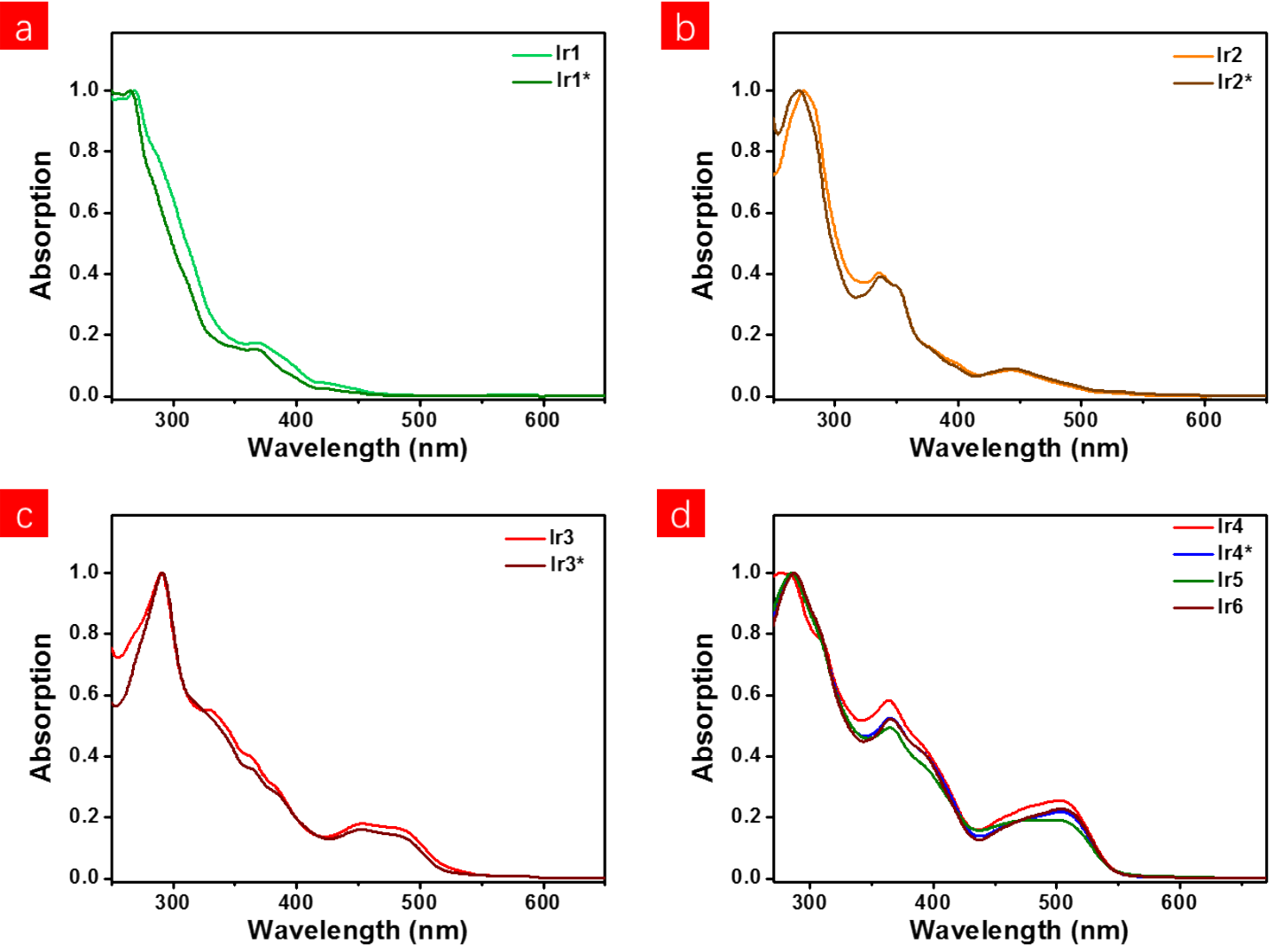

Figure S1. Normalized absorption spectra of phosphorescent iridium(III) complex probes and corresponding model complexes in PBS (pH 7.4, 10\% $\mathrm{CH}_{3} \mathrm{CN}$ ). (a) Ir1 and Ir1*, (b) Ir2 and Ir2*, (c) Ir3 and Ir 3*, (d) Ir4, Ir4*, Ir5 and Ir6. 

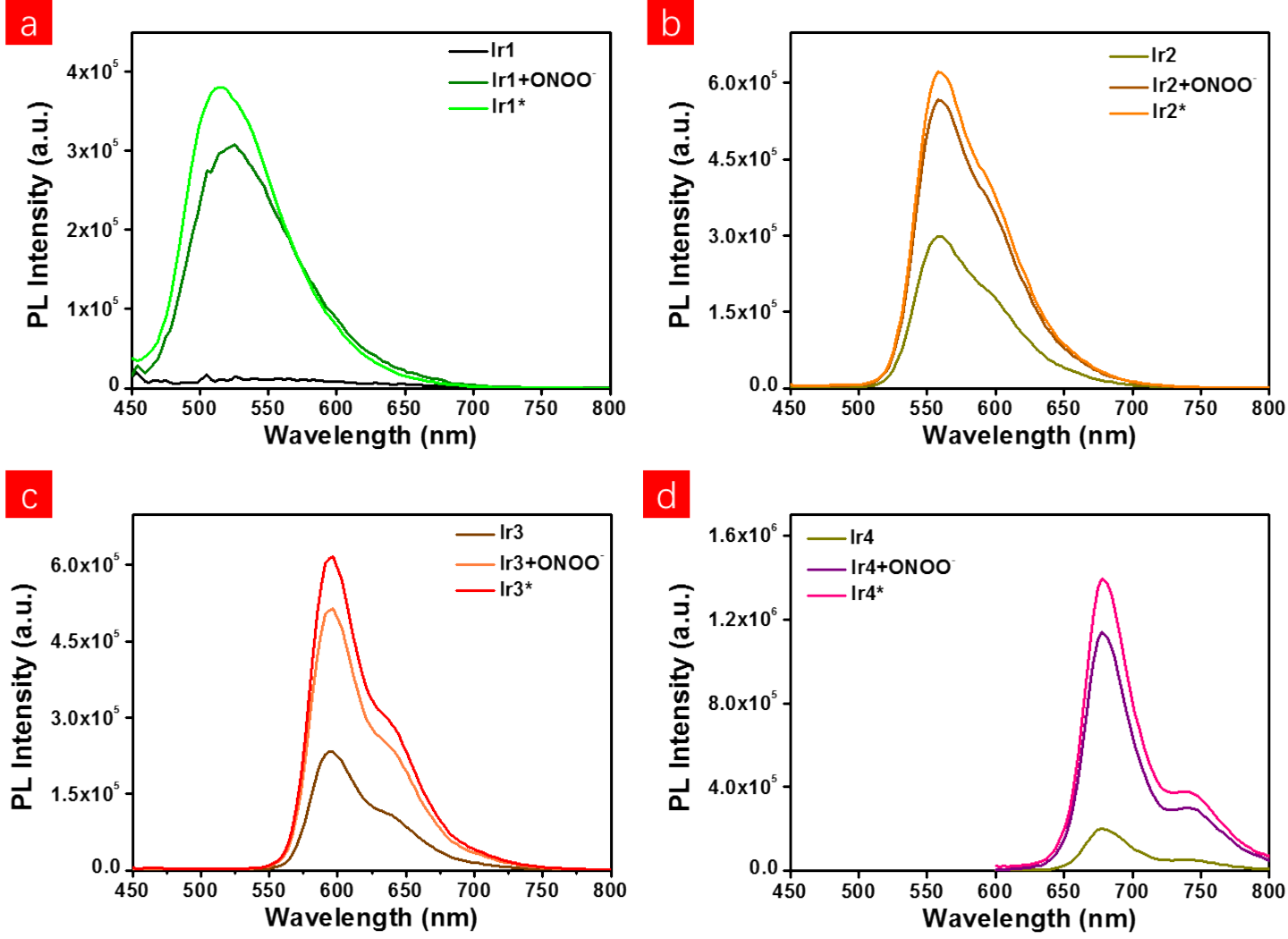

Figure S2. Emission spectra of iridium(III) complex probes upon addition of $\mathrm{ONOO}^{-}(20 \mu \mathrm{M})$ and corresponding model complexes $(5 \mu \mathrm{M})$ in PBS ( $\left.\mathrm{pH} 7.4,10 \% \mathrm{CH}_{3} \mathrm{CN}\right)$. (a) Ir1, Ir1 + $\mathrm{ONOO}^{-}$and Ir1*, (b) Ir2, Ir2 + $\mathrm{ONOO}^{-}$and Ir2*, (c) Ir3, Ir3 $+\mathrm{ONOO}^{-}$and Ir ${ }^{*}$, (d) Ir4, Ir4 $+\mathrm{ONOO}^{-}$and $\mathbf{I r} 4^{*}$. 

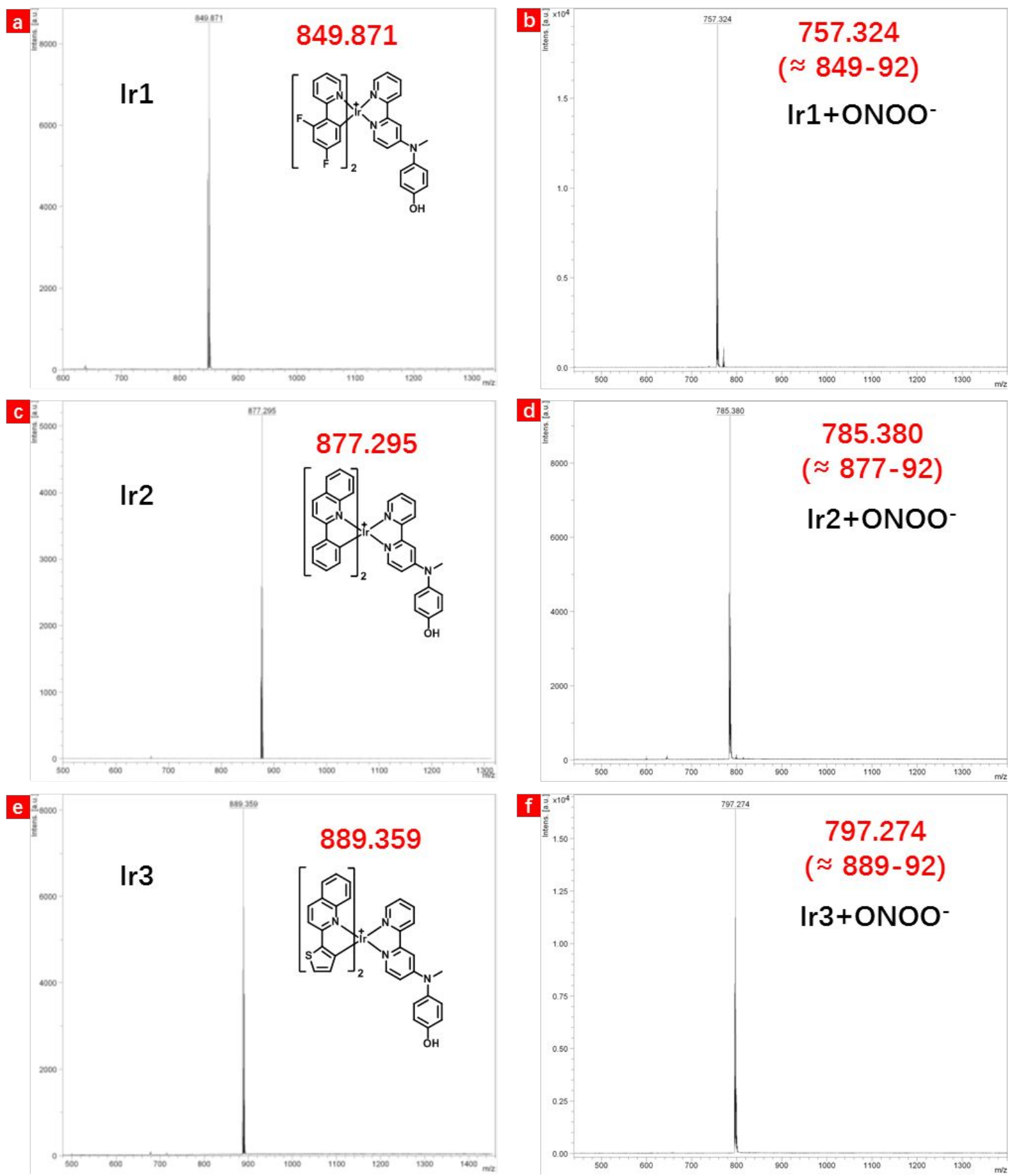

Figure S3. The MALDI-TOF-MS spectra of iridium(III) complex probes Ir1 (a), Ir2 (c) and Ir3 (e); The MALDI-TOF-MS spectra of iridium(III) complex probes incubated with excessive $\mathrm{ONOO}^{-}$for 5 min: Ir1 + $\mathrm{ONOO}^{-}$(b), Ir2 + $\mathrm{ONOO}^{-}$(d) and Ir3 $+\mathrm{ONOO}^{-}$(f). 


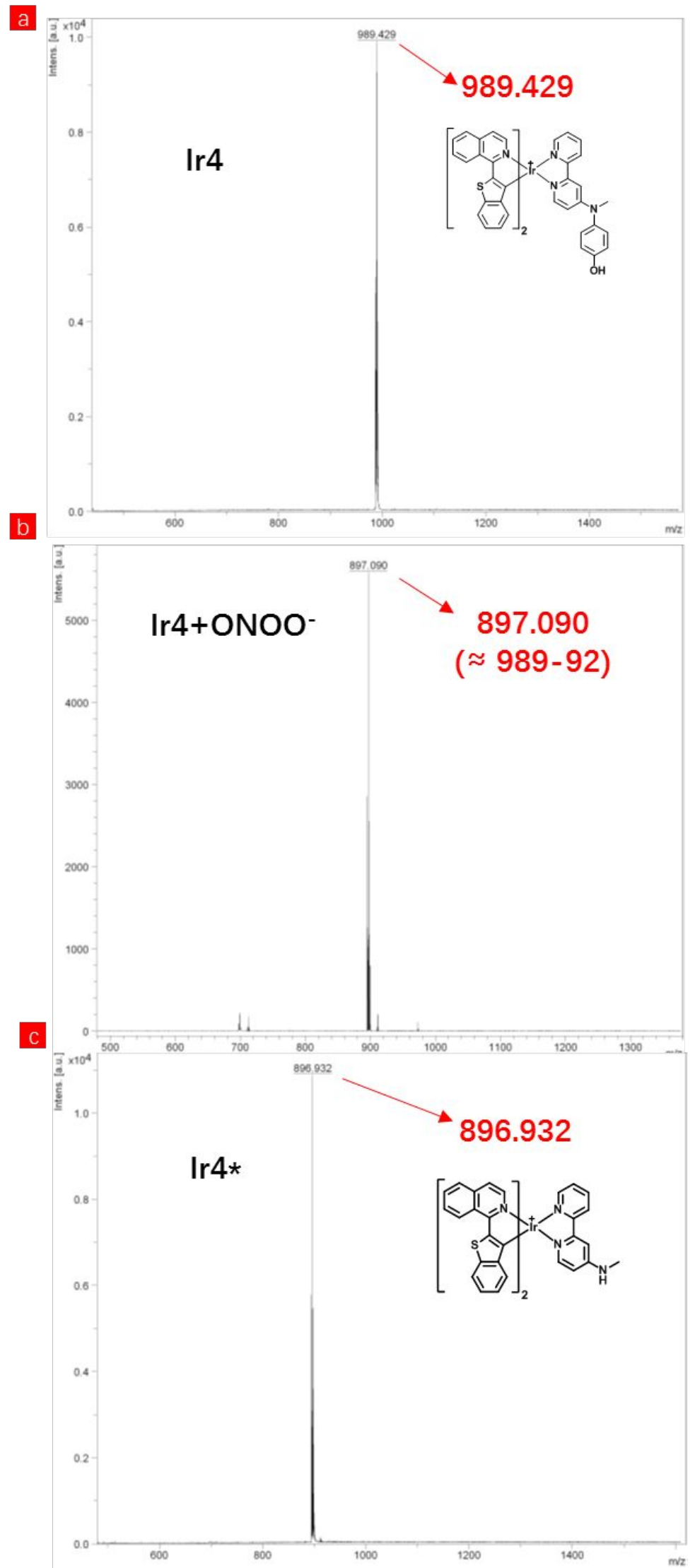

Figure S4. (a) The MALDI-TOF-MS spectra of iridium(III) complex probe Ir4; (b) The MALDI-TOF-MS spectra of probe Ir4 incubated with excessive $\mathrm{ONOO}^{-}$for $5 \mathrm{~min}$; (c) The MALDI-TOF-MS spectra of model iridium(III) complex Ir4*. 


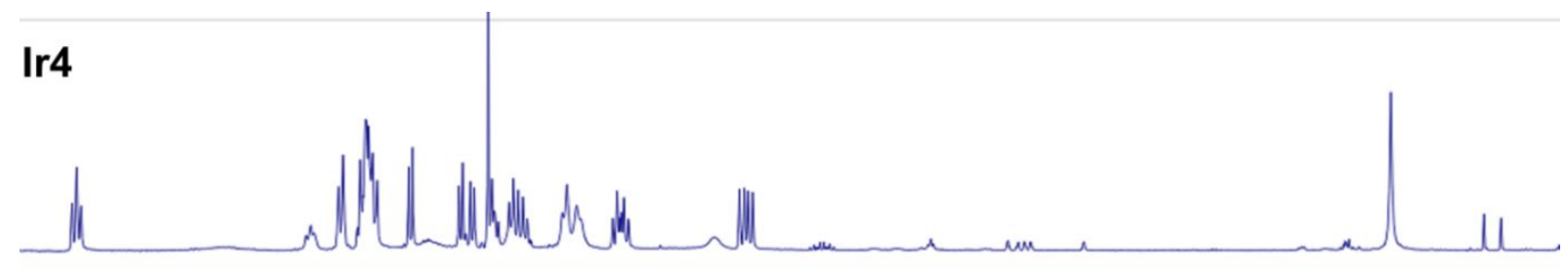

Ir4+ONOO-

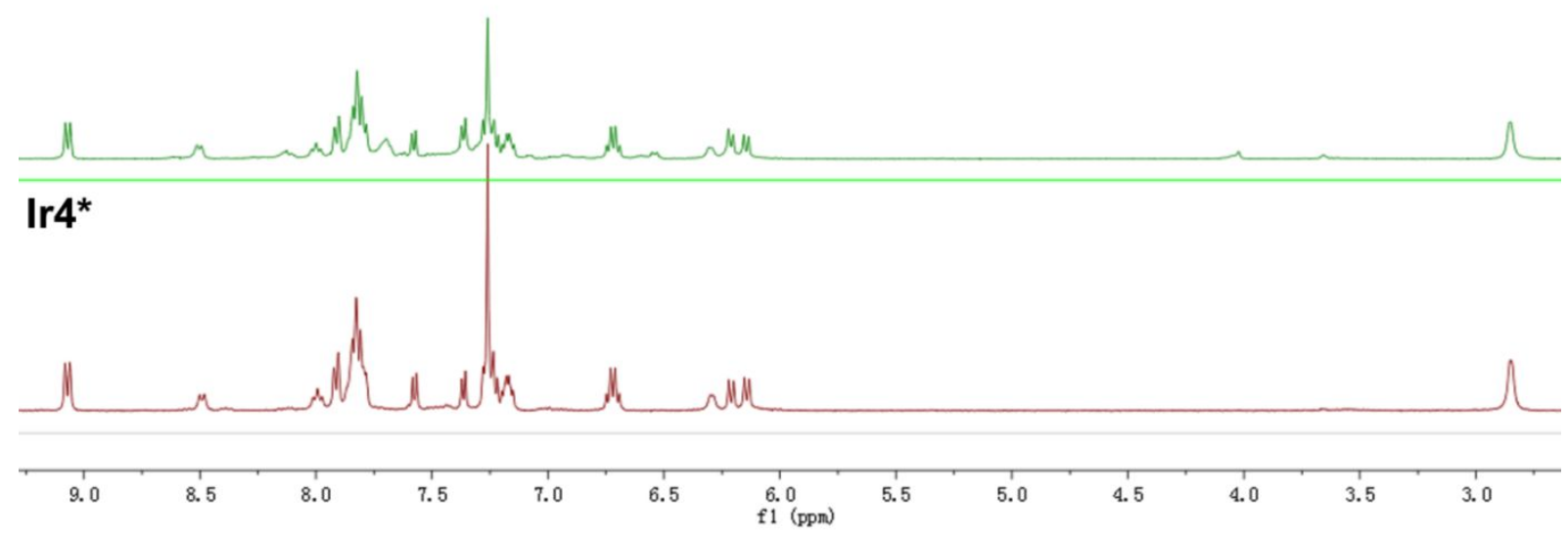

Figure S5. The ${ }^{1} \mathrm{H}$ NMR spectra of iridium(III) complex probe Ir4, main product of probe Ir4 reacting with excessive ONOO- and model iridium(III) complex Ir4*. 

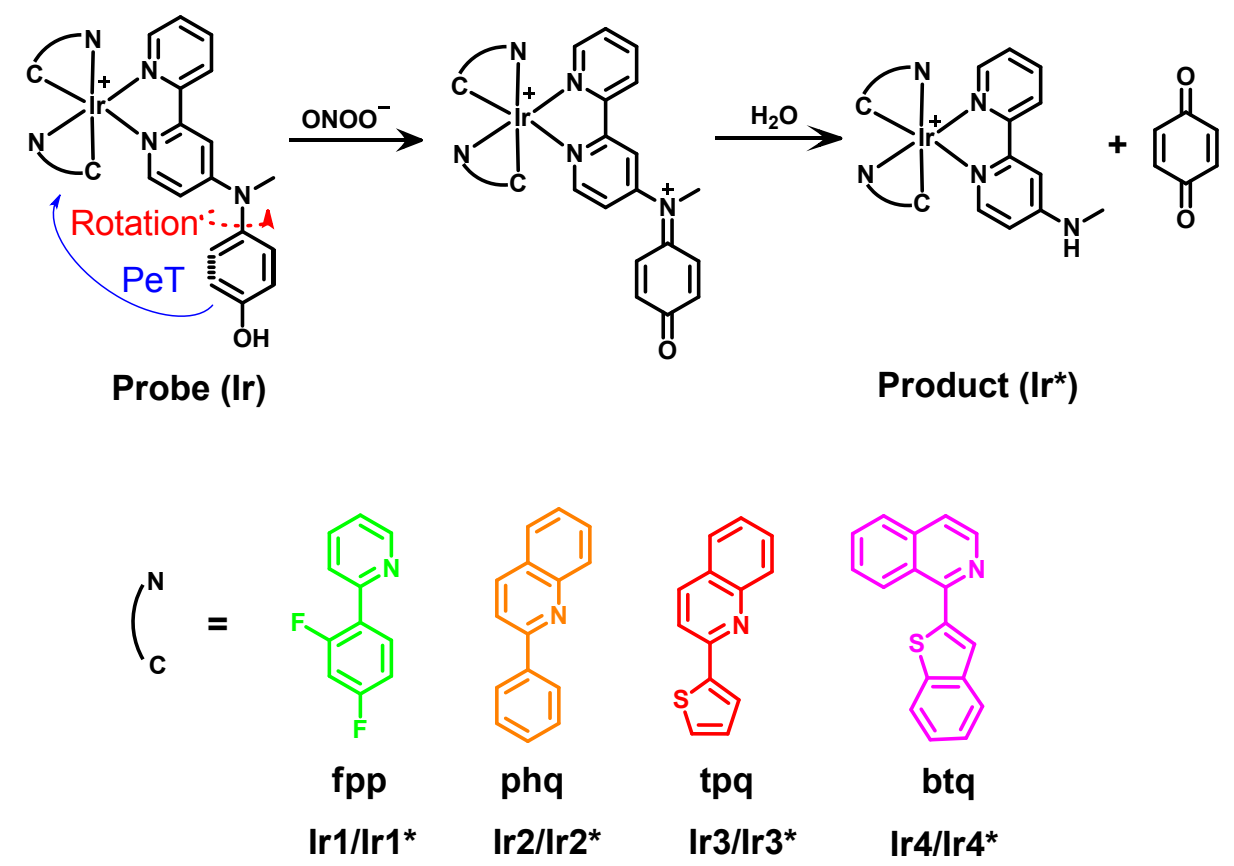

Figure S6. Proposed mechanisms of reactions between iridium(III) complex probes and ONOO- 

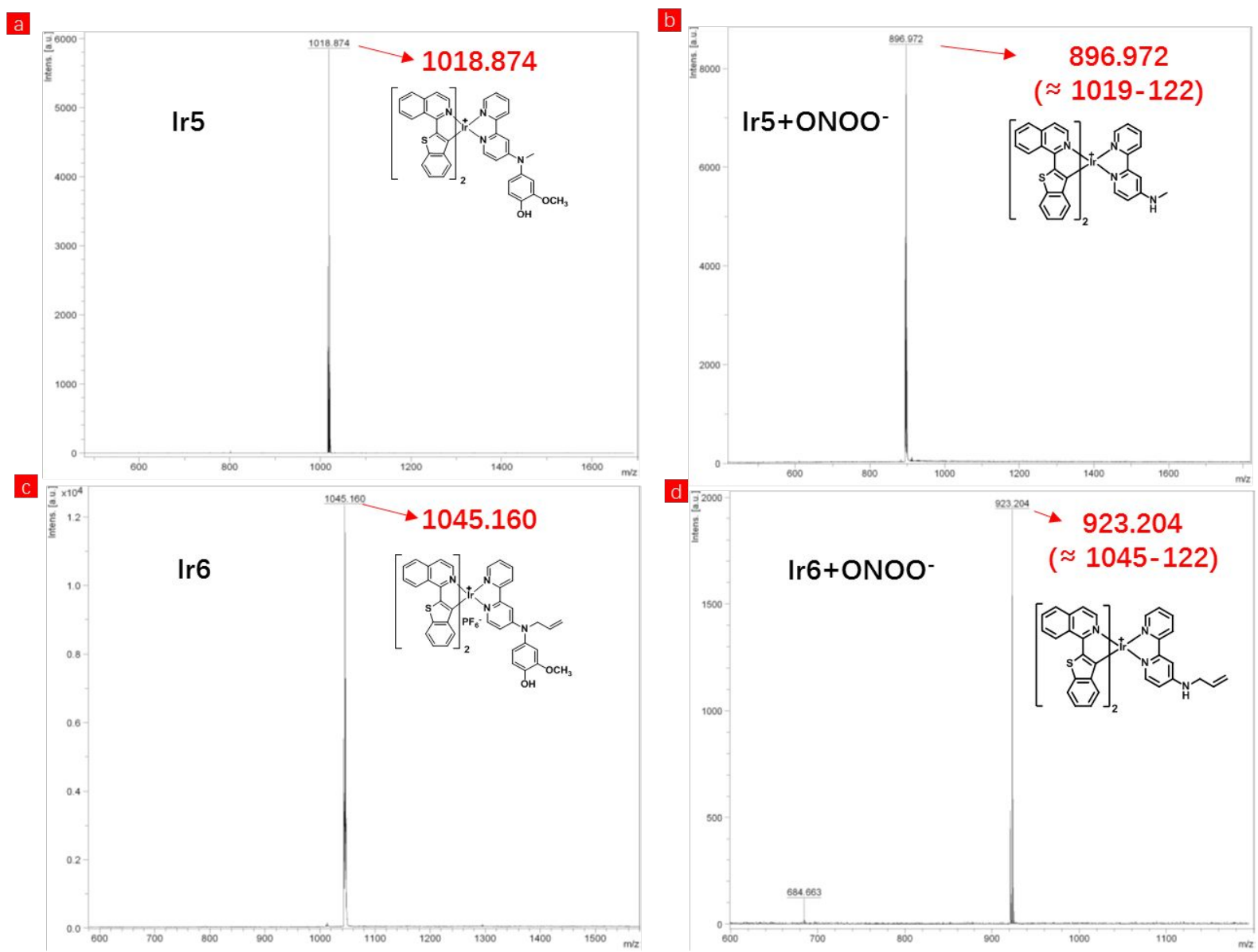

Figure S7. The MALDI-TOF-MS spectra of iridium(III) complex probe Ir5 (a) and probe Ir5 incubated with excessive $\mathrm{ONOO}^{-}(\mathrm{b})$; The MALDI-TOF-MS spectra of iridium(III) complex probe Ir6 (c) and probe Ir6 incubated with excessive $\mathrm{ONOO}^{-}(\mathrm{d})$. 


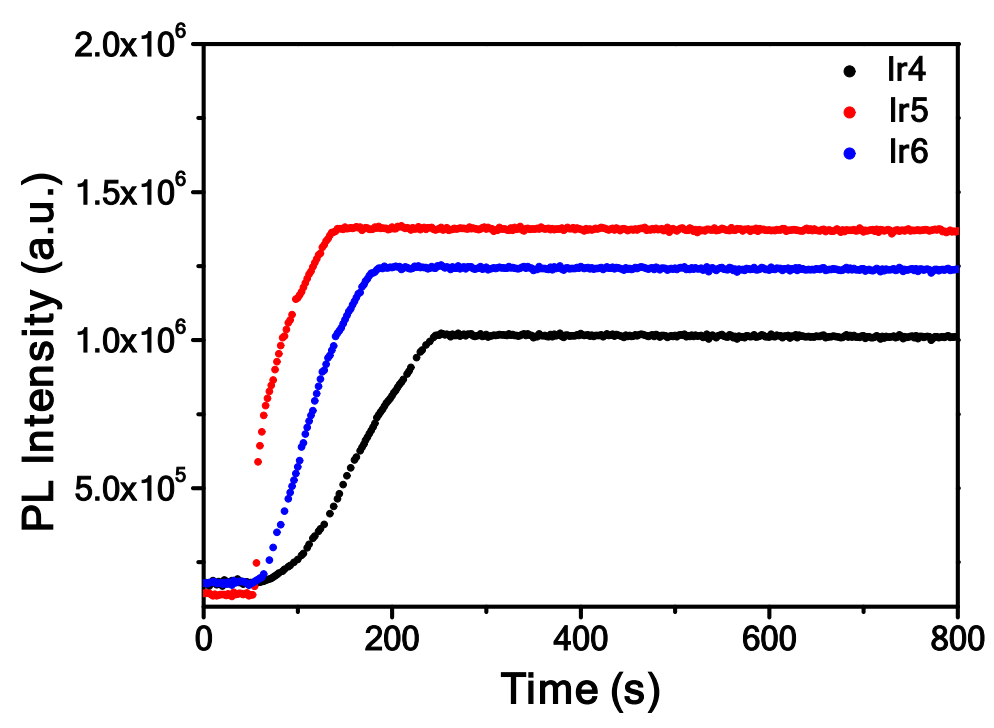

Figure S8. Time course in the detection of $\mathrm{ONOO}^{-}(20 \mu \mathrm{M})$ with $\operatorname{Ir4}$, Ir5 and $\operatorname{Ir6}(5 \mu \mathrm{M})$ in PBS $\left(10 \% \mathrm{CH}_{3} \mathrm{CN}\right)$ monitored by photoluminescence, respectively. The photoluminescence intensity at $680 \mathrm{~nm}$ was recorded. 


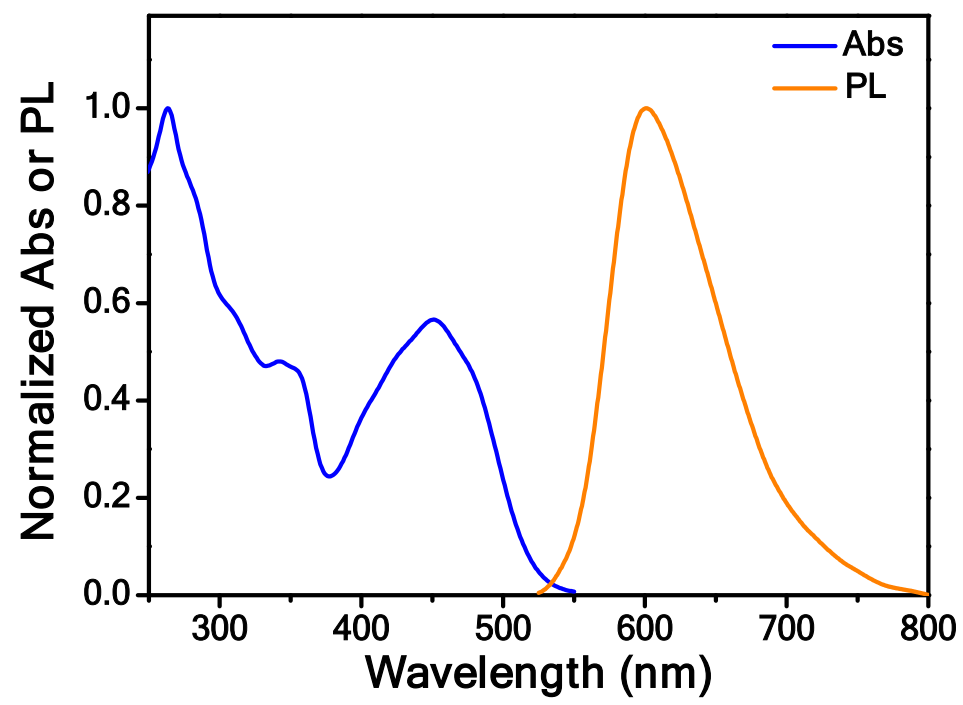

Figure S9. Normalized absorption and emission spectra of phosphorescent iridium complex Ir7 in PBS $\left(10 \% \mathrm{CH}_{3} \mathrm{CN}\right)$. 


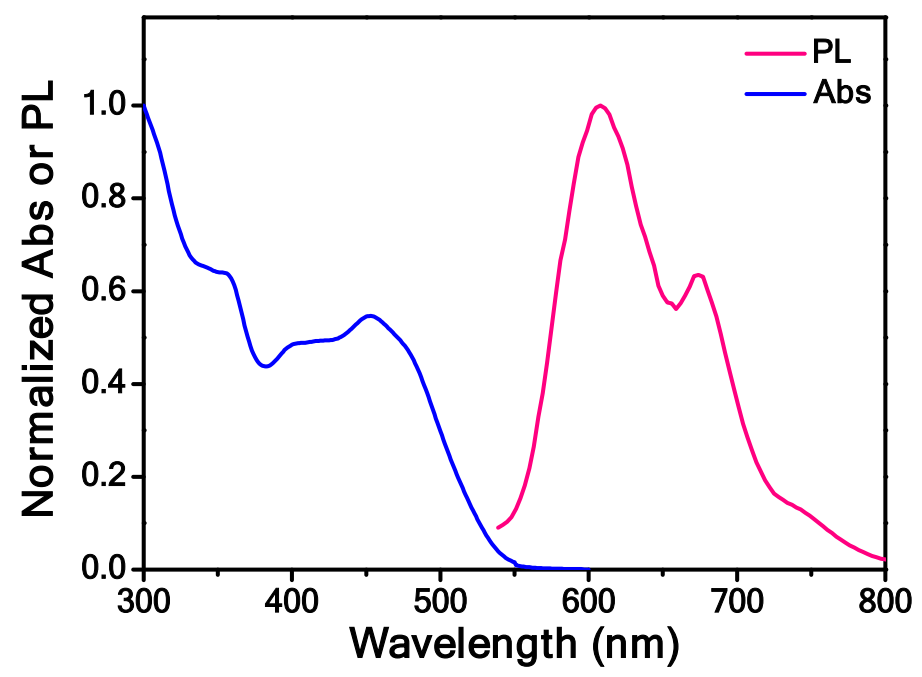

Figure S10. Normalized absorption and emission spectra of P-ONOO $\left(0.1 \mathrm{mg} \mathrm{mL}^{-1}\right)$ in PBS. 
a
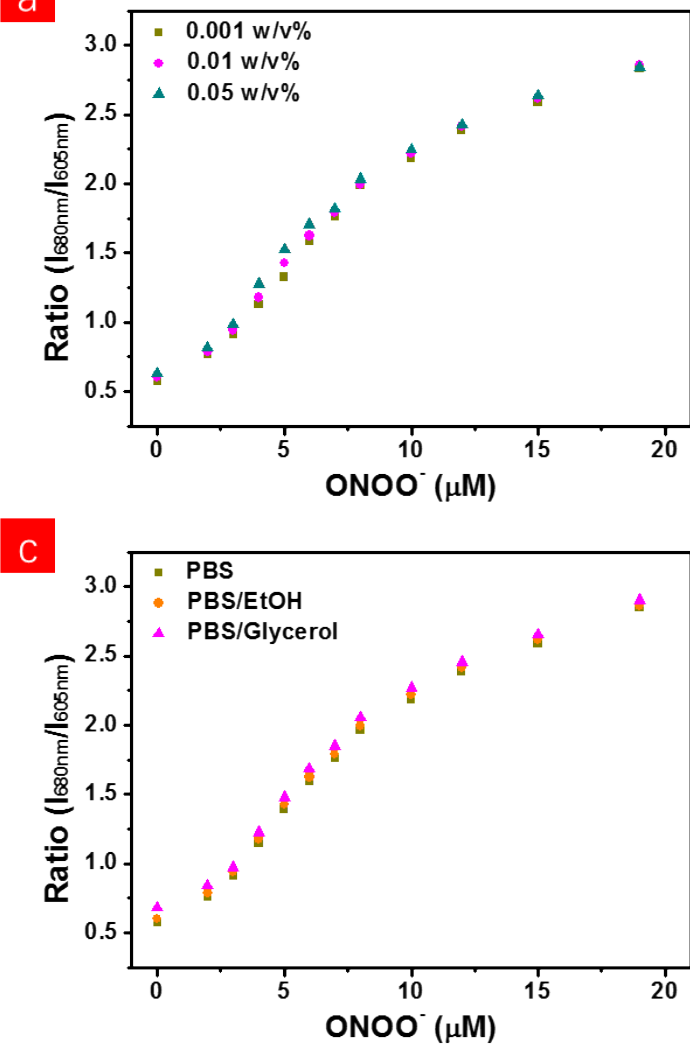

b
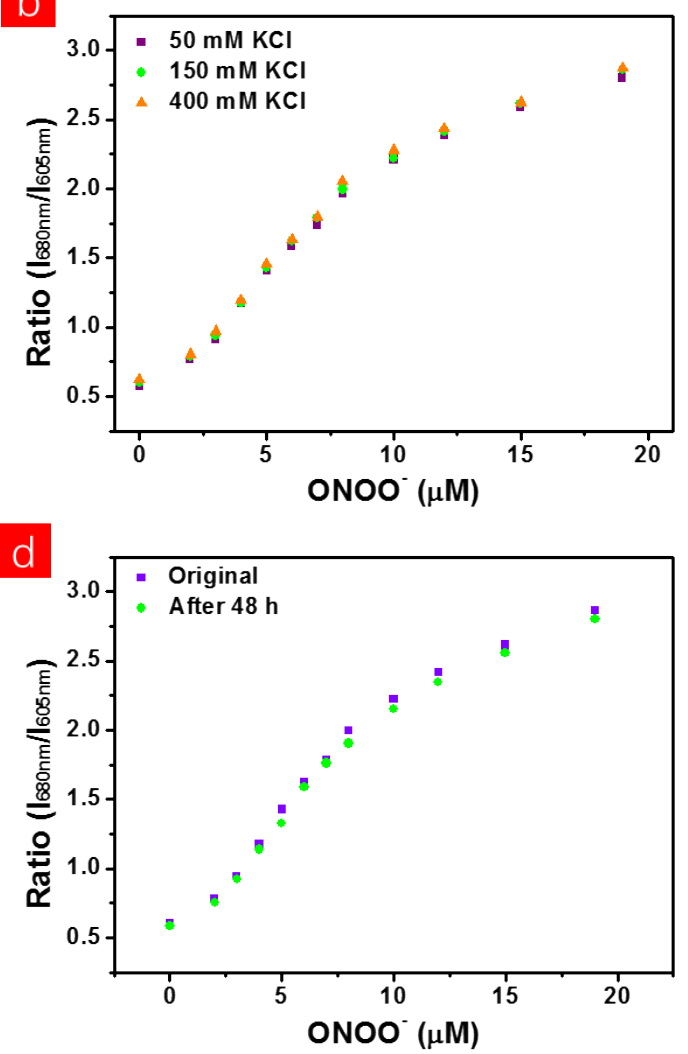

Figure S11. Effects of concentration (a), ionic strength (b), viscosity (c) and freshness (d) on the phosphorescence ratio of P-ONOO. Excitation wavelength was $515 \mathrm{~nm}$. 

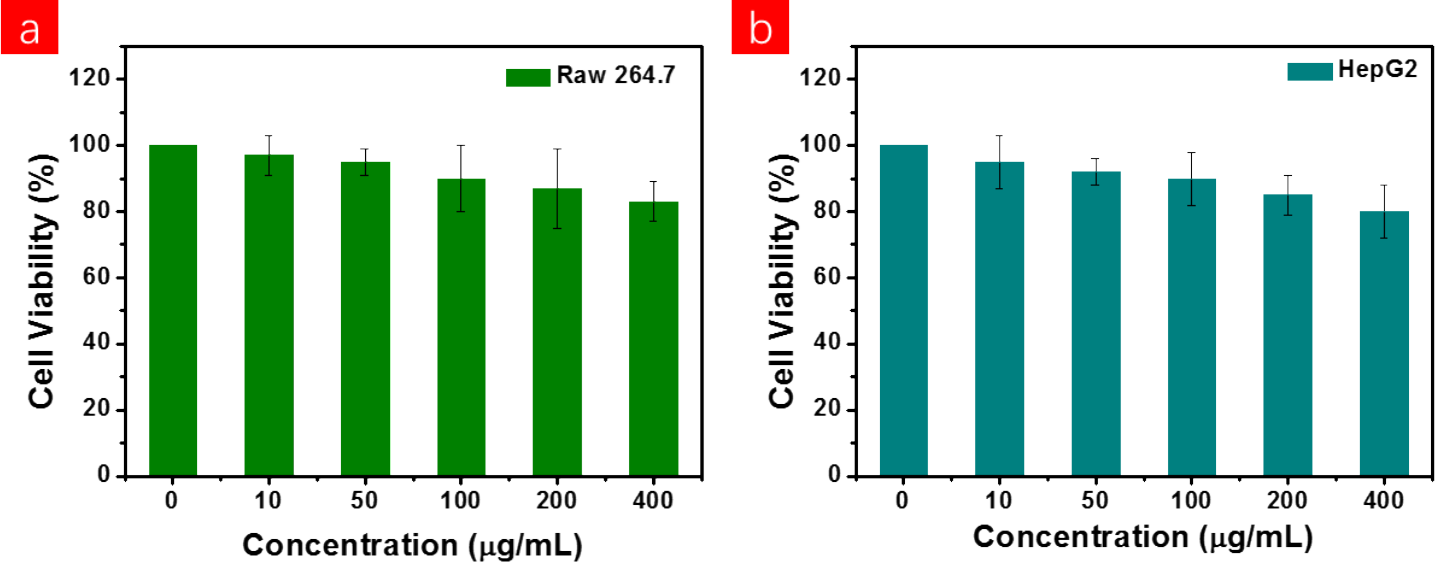

Figure S12. Cytotoxicity of P-ONOO on RAW264.7 cells (a) and HepG2 cells (b) determined by MTT assay. 

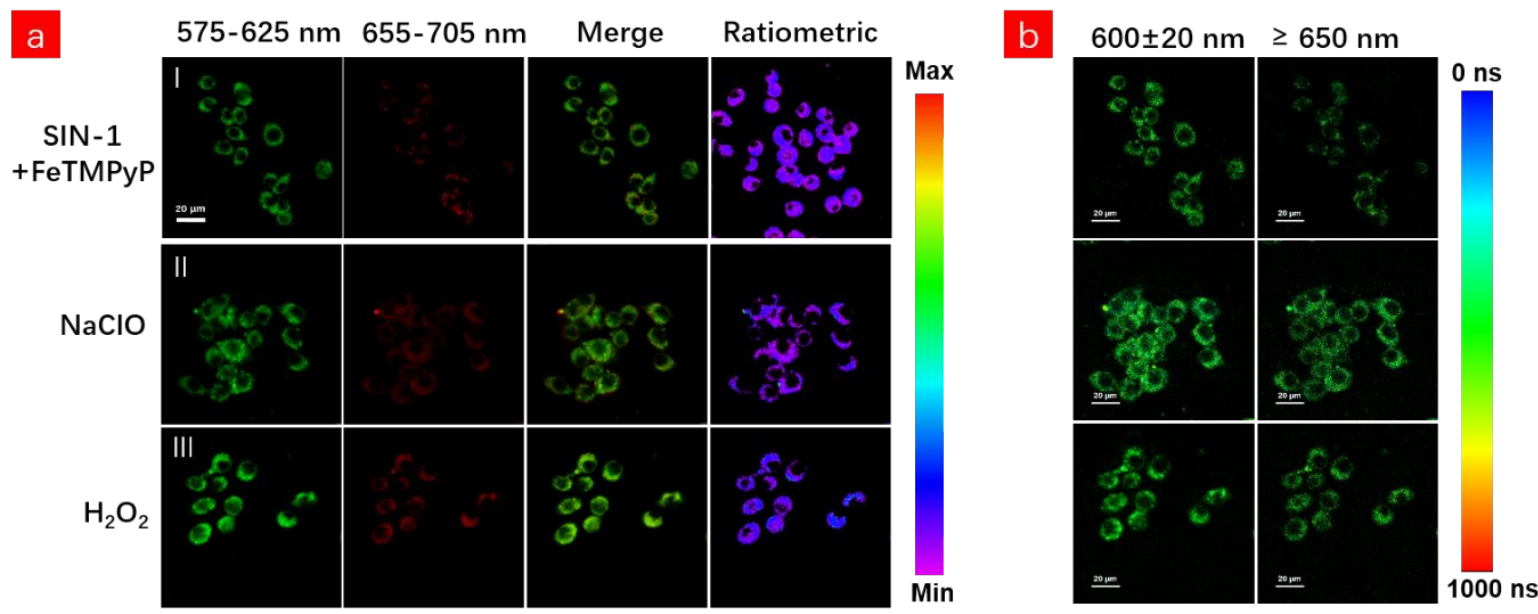

Figure S13. (a) The CLSM images and ratiometric photoluminescence images of living RAW264.7 cells labeled with P-ONOO for $2 \mathrm{~h}$ under different conditions: (I) cells pretreated with peroxynitrite scavenger FeTMPyP $(50 \mu \mathrm{M})$ and subsequently incubated with SIN-1 (100 $\mu \mathrm{M})$; (II) cells subsequently incubated with $\mathrm{ClO}^{-}(100 \mu \mathrm{M})$; (III) cells subsequently incubated with $\mathrm{H}_{2} \mathrm{O}_{2}(100 \mu \mathrm{M})$. The green channels were acquired by collecting the photoluminescence from 575 to $625 \mathrm{~nm}$, while the red channels were from 655 to $705 \mathrm{~nm}$. The ratiometric photoluminescence images were the red channel to green channel and excitation wavelength was $515 \mathrm{~nm}$. (b) The related PLIM images of living P-ONOO-stained RAW 264.7 cells depicted in (a). Photoluminescence was collected through bandpass filter $(600 \pm 20 \mathrm{~nm})$ or longpass filter ( $\geq 650 \mathrm{~nm}$ ) and excitation wavelength was $405 \mathrm{~nm}$. The scale bar is $20 \mu \mathrm{m}$. 

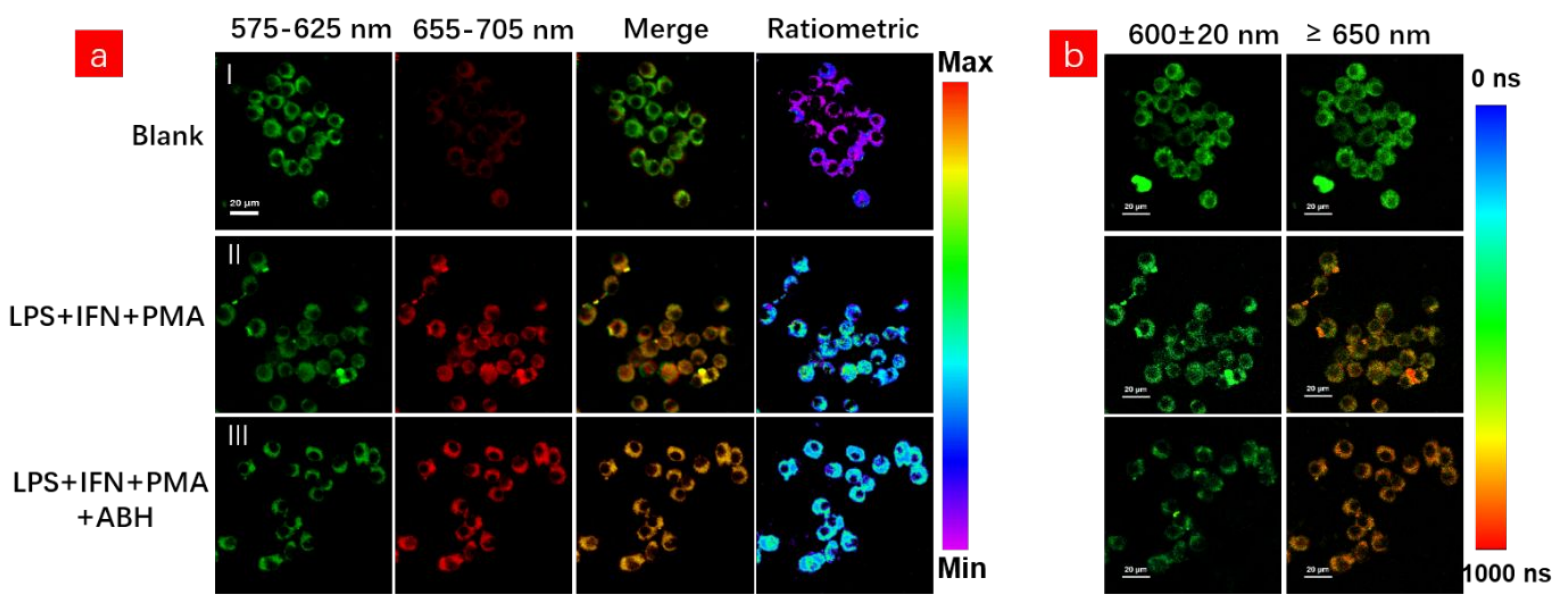

Figure S14. (a) The CLSM images and ratiometric photoluminescence images of living RAW264.7 cells labeled with P-ONOO for $2 \mathrm{~h}$ under different conditions: (I) Cells without prestimulation; (II) Cells were prestimulated with LPS $\left(1 \mu \mathrm{g} \mathrm{mL}^{-1}\right)$, IFN- $\Upsilon\left(50 \mathrm{ng} \mathrm{mL}^{-1}\right)$ for 12 $\mathrm{h}$ and PMA (20 nM) for $0.5 \mathrm{~h}$; (III) Cells pretreated with LPS/IFN- $\Upsilon$ /PMA and hypochlorite scavenger $\mathrm{ABH}(100 \mu \mathrm{M})$ for $12 \mathrm{~h}$. The green channels were acquired by collecting the photoluminescence from 575 to $625 \mathrm{~nm}$, while the red channels were from 655 to $705 \mathrm{~nm}$. The ratiometric photoluminescence images were the red channel to green channel and excitation wavelength was $515 \mathrm{~nm}$. (b) The related PLIM images of living P-ONOO-stained RAW 264.7 cells depicted in (a). Photoluminescence was collected through bandpass filter (600 $\pm 20 \mathrm{~nm})$ or longpass filter ( $\geq 650 \mathrm{~nm}$ ) and excitation wavelength was $405 \mathrm{~nm}$. The scale bar is $20 \mu \mathrm{m}$. 

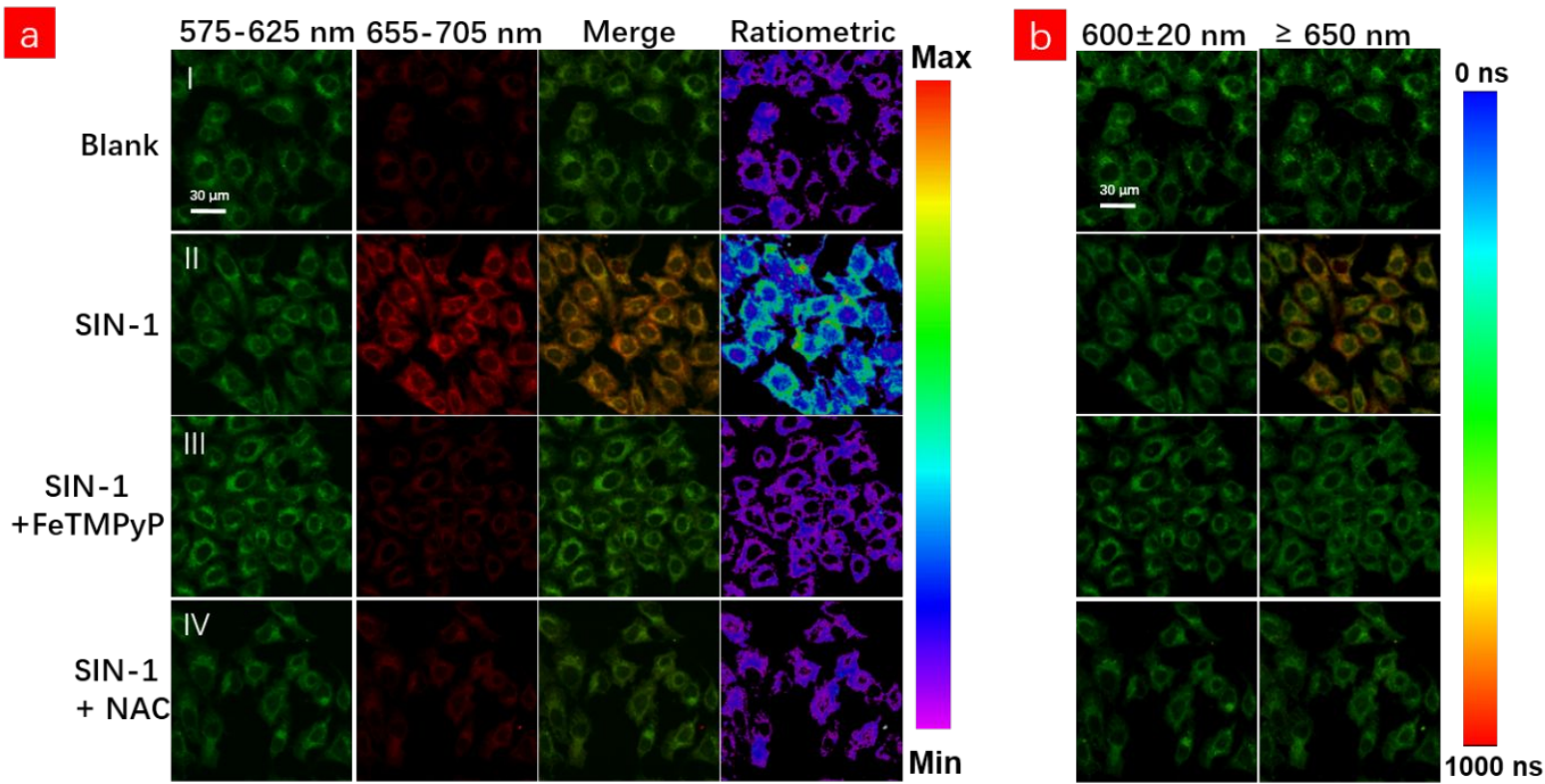

Figure S15. The CLSM images and ratiometric photoluminescence images of living HepG2 cells under different conditions: (I) HepG2 cells labeled with P-ONOO only; (II) P-ONOOstained cells were subsequently incubated with SIN-1 (100 $\mu \mathrm{M})$; (III) P-ONOO-stained cells pretreated with peroxynitrite scavenger FeTMPyP $(50 \mu \mathrm{M})$ and subsequently incubated with SIN-1 $(100 \mu \mathrm{M})$; (IV) P-ONOO-stained cells pretreated with NAC (5 mM) for $1 \mathrm{~h}$ and subsequently incubated with SIN-1 $(100 \mu \mathrm{M})$. The green channels were acquired by collecting the photoluminescence from 575 to $625 \mathrm{~nm}$, while the red channels were from 655 to $705 \mathrm{~nm}$ and excitation wavelength was $515 \mathrm{~nm}$. (b) The related PLIM images of living P-ONOOstained HepG2 cells depicted in (a). Photoluminescence was collected through bandpass filter $(600 \pm 20 \mathrm{~nm})$ or longpass filter $(\geq 650 \mathrm{~nm})$ and excitation wavelength was $405 \mathrm{~nm}$. The scale bar is $30 \mu \mathrm{m}$. 


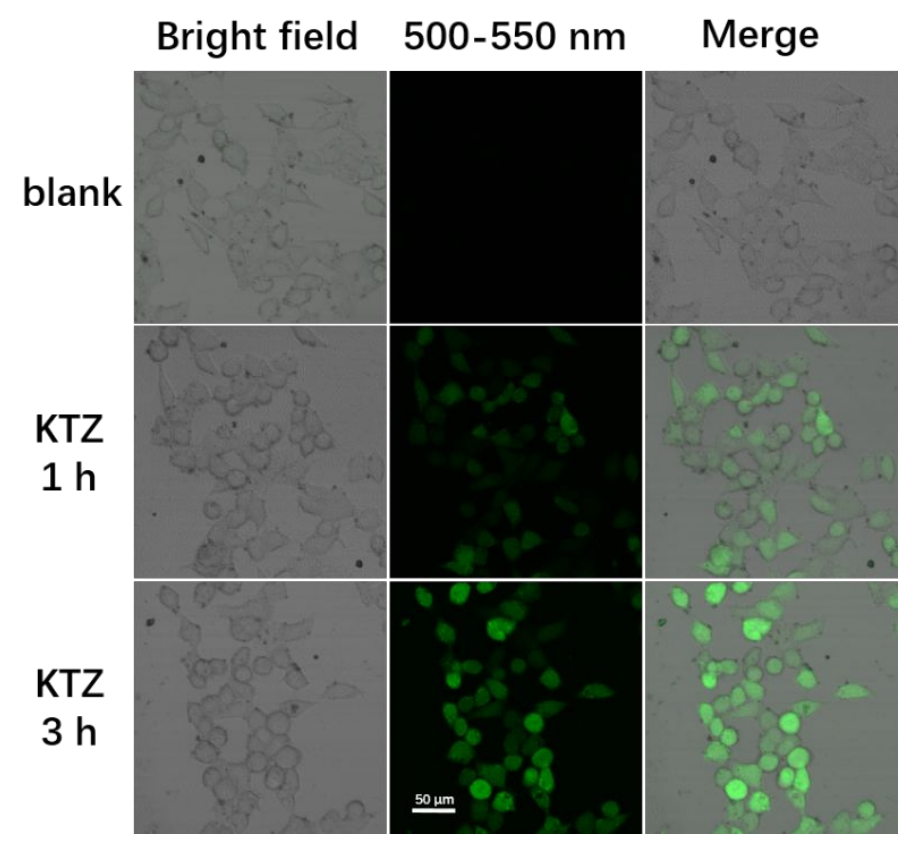

Figure S16. The CLSM images of HepG2 cells pretreated with KTZ $(25 \mu \mathrm{M})$ for $1,3 \mathrm{~h}$ or not. The green channels were acquired by collecting the photoluminescence from 500 to $550 \mathrm{~nm}$ and excitation wavelength was $488 \mathrm{~nm}$. The production of intracellular ROS was determined by measuring the conversion of the non-fluorescent probe 2',7'-dichlorodihydrofluorescein diacetate $(\mathrm{DCFH})$ to the fluorescent metabolite 2',7'-dichlorofluorescein. The scale bar is 50 $\mu \mathrm{m}$. 


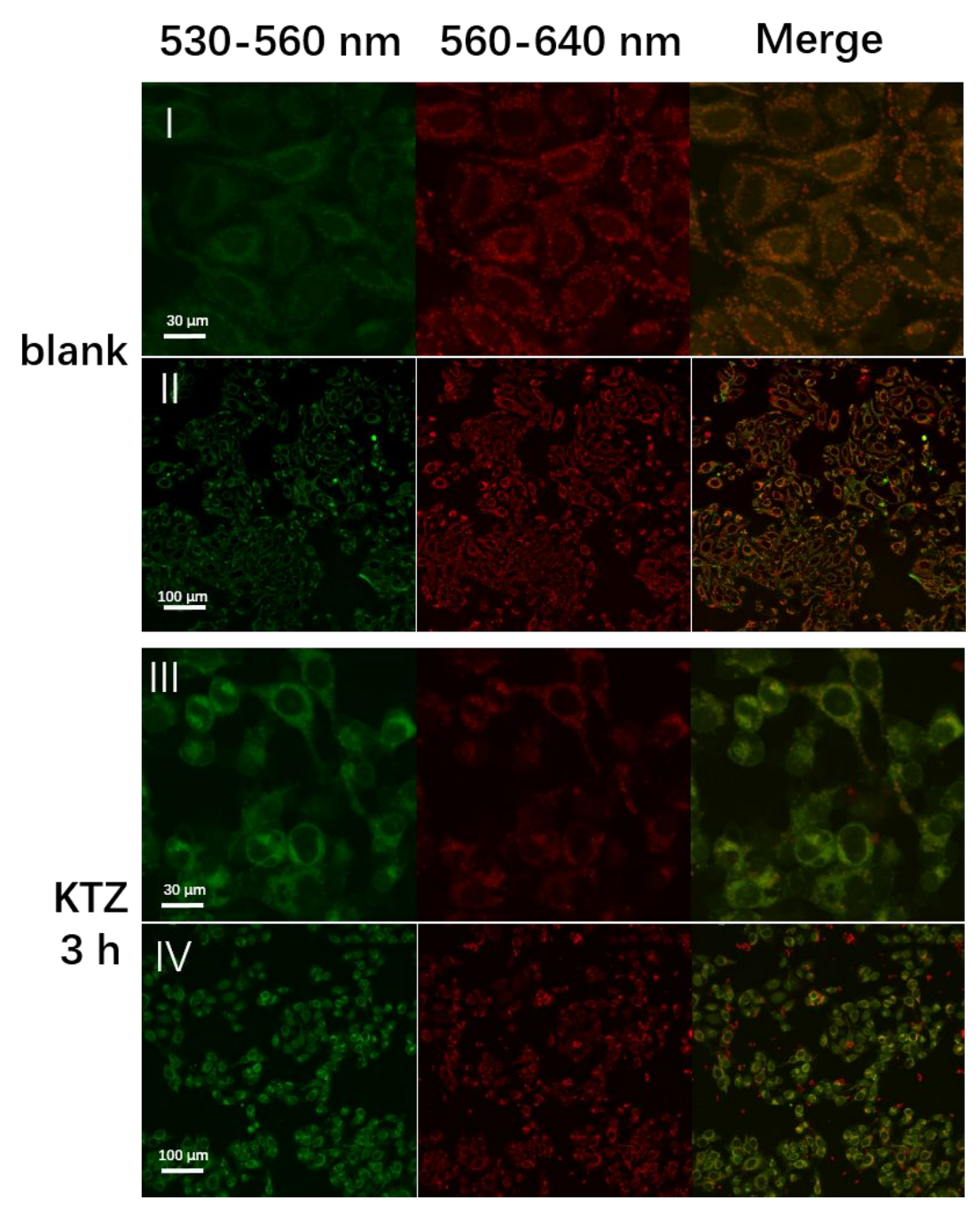

Figure S17. Effects of ketoconazole (KTZ) on mitochondria of HepG2 cells. The CLSM images of JC-1 stained HepG2 cells pretreated with KTZ $(10 \mu \mathrm{M})$ for $3 \mathrm{~h}$ (III, IV) or not (I, II). The green channels were acquired by collecting the photoluminescence from 530 to $560 \mathrm{~nm}$, while the red channels were from 560 to $640 \mathrm{~nm}$ and excitation wavelength was $488 \mathrm{~nm}$. Since early apoptotic cells undergo characteristic depolarization of the mitochondria, the mitochondrial membrane potentials (MMP) were measured using JC-1 as an indicator, which was converted from aggregation state to monomer upon decreasing MMP accompanied with a fluorescence color change from red to green. The scale bar are $30 \mu \mathrm{m}$ and $100 \mu \mathrm{m}$, respectively. 


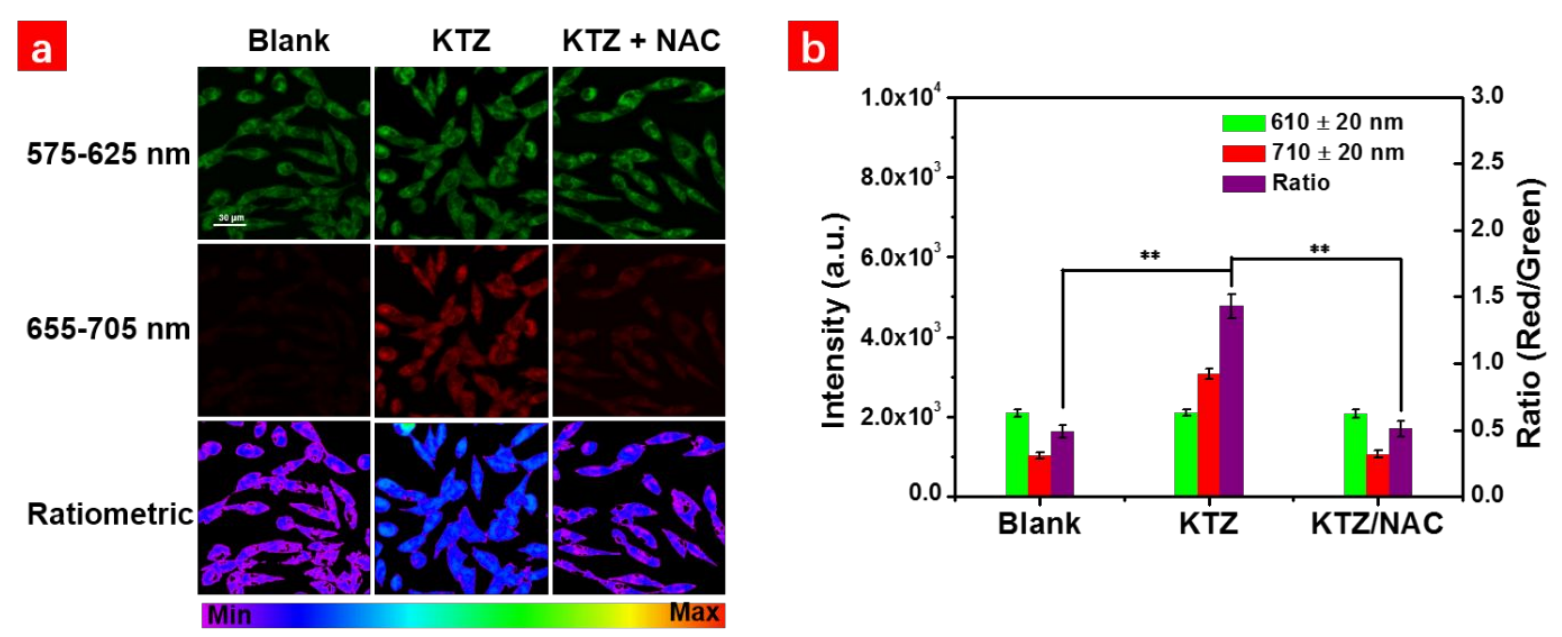

Figure S18. P-ONOO for endogenous $\mathrm{ONOO}^{-}$detection in living HL7702 cells by ratiometric photoluminescence imaging. (a) CLSM images and ratiometric photoluminescence images of living HL7702 cells labeled with P-ONOO for $2 \mathrm{~h}$ under different conditions: (I) Cells without prestimulation; (II) Cells pretreated with KTZ $(10 \mu \mathrm{M})$ for $3 \mathrm{~h}$; (III) Cells pretreated with NAC $(5 \mathrm{mM})$ for $1 \mathrm{~h}$ and co-incubated with $\mathrm{KTZ}(10 \mu \mathrm{M})$ for another $3 \mathrm{~h}$. The green channels were acquired by collecting the photoluminescence from 575 to $625 \mathrm{~nm}$, while the red channels were from 655 to $705 \mathrm{~nm}$. The scale bar is $30 \mu \mathrm{m}$. (b) Photoluminescence intensity of RAW 264.7 cells recorded from the green channel (green) and the red channel (red) and the intensity ratio (purple). ${ }^{* *}: \mathrm{p}<0.01(\mathrm{n}=3)$. 

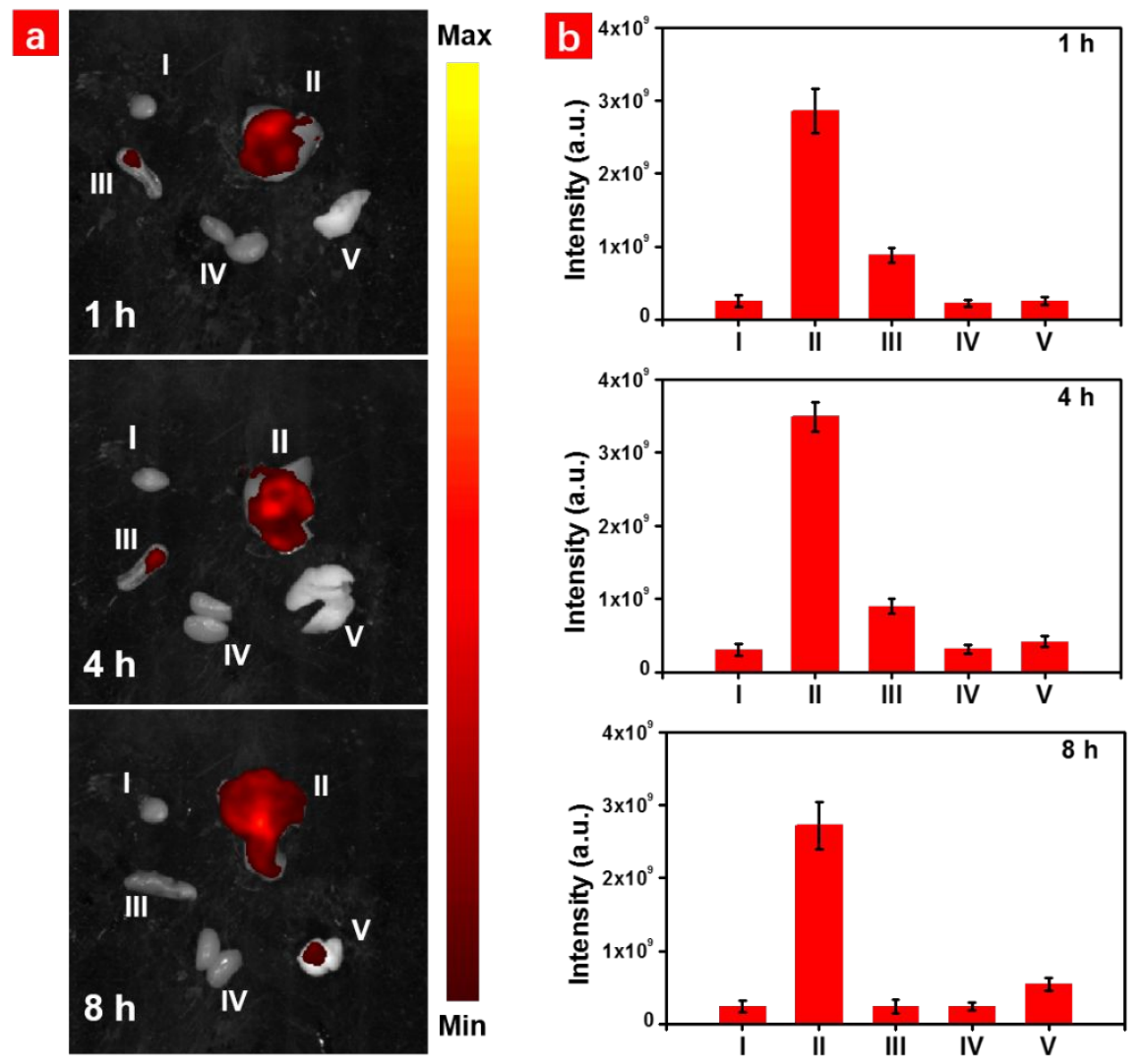

Figure S19. (a) Ex vivo images of major organs dissected after sacrificing mice at $1 \mathrm{~h}, 4 \mathrm{~h}$, and $8 \mathrm{~h}$ after intravenously injected with P-ONOO $\left(50 \mu \mathrm{L}, 5 \mathrm{mg} \mathrm{kg}^{-1}\right)$. I, Heart; II, Liver; III, Spleen; IV, Lung; V, Kidney. (b) The photoluminescence intensity of each organ in (a). Excitation wavelength was $520 \mathrm{~nm}$ and the emission band was at 600-700 nm. 

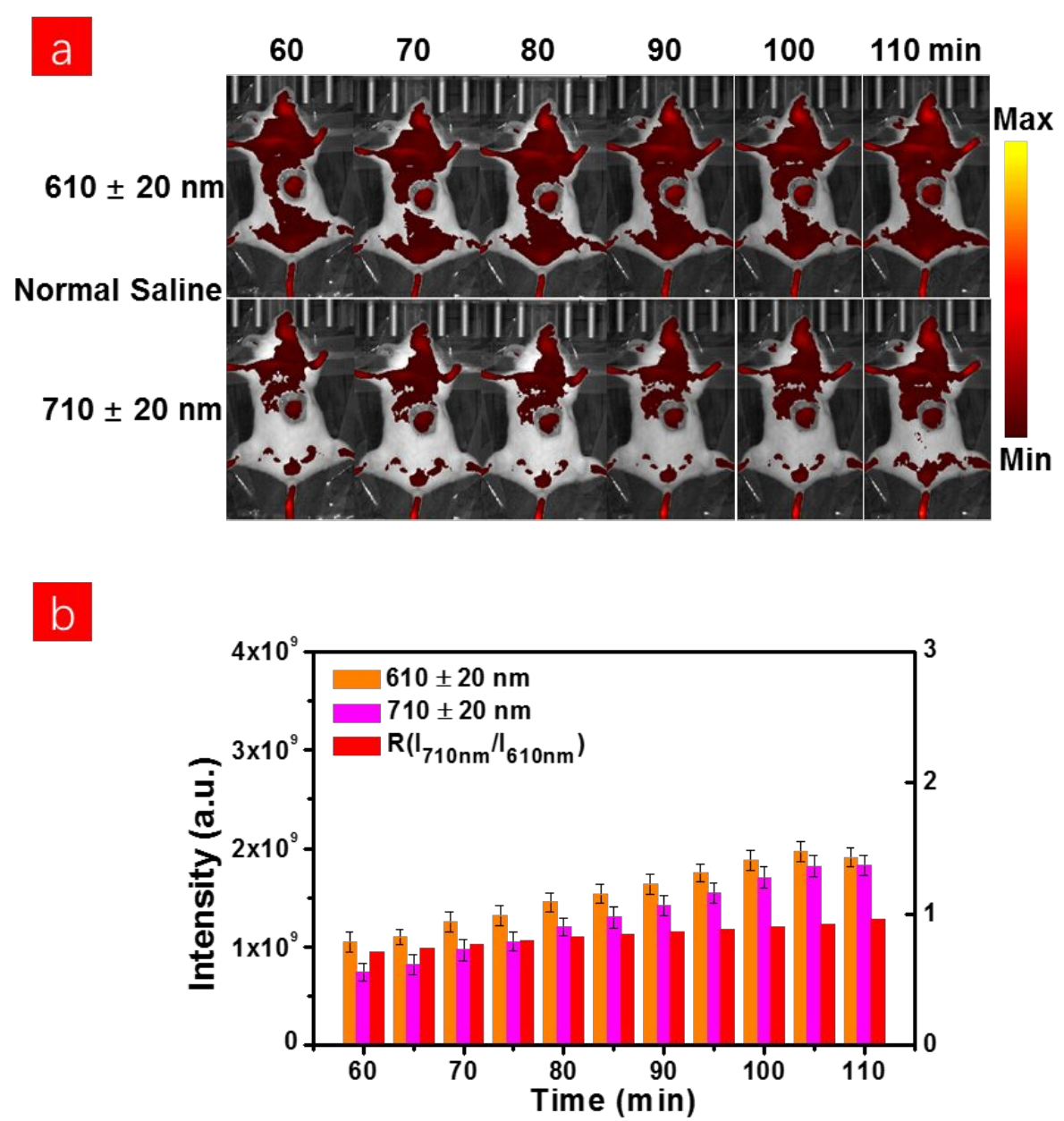

Figure S20. (a) Photoluminescence imaging of liver-exposed mice. The mice injected with normal saline intraperitoneally and subsequently injected with P-ONOO intravenously. The images were captured every $5 \mathrm{~min}$ from the short-wavelength channel of $610 \pm 20 \mathrm{~nm}$ and longwavelength channel of $710 \pm 20 \mathrm{~nm}$ after administration of saline solutions for $1 \mathrm{~h}$. Excitation wavelength was $510 \mathrm{~nm}$. (b) Time-dependent mean photouminescence intensity of exposed livers recorded from the short-wavelength channel (orange), the long-wavelength channel (pink) and the intensity ratio (red). 

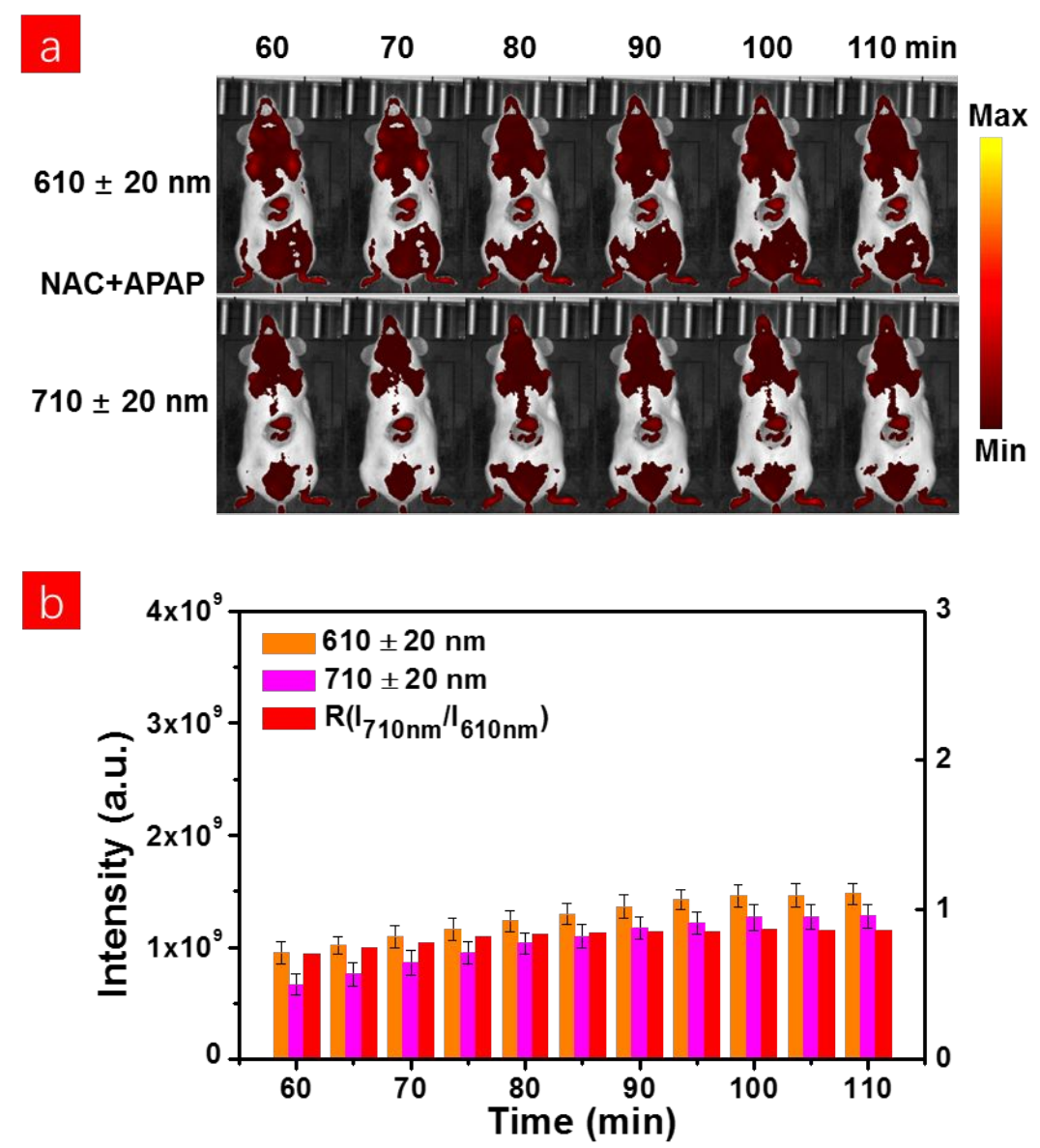

Figure S21. (a) Photoluminescence imaging of liver-exposed mice. The mice were injected with NAC (300 mg kg-1) intraperitoneally $1 \mathrm{~h}$ before injected with APAP (300 mg kg-1) intraperitoneally and P-ONOO intravenously. The images were captured every $5 \mathrm{~min}$ from the short-wavelength channel of $610 \pm 20 \mathrm{~nm}$ and long-wavelength channel of $710 \pm 20 \mathrm{~nm}$ after administration of APAP for $1 \mathrm{~h}$. Excitation wavelength was $510 \mathrm{~nm}$. (b) Time-dependent mean photouminescence intensity of exposed livers recorded from the short-wavelength channel (orange), the long-wavelength channel (pink) and the intensity ratio (red). 


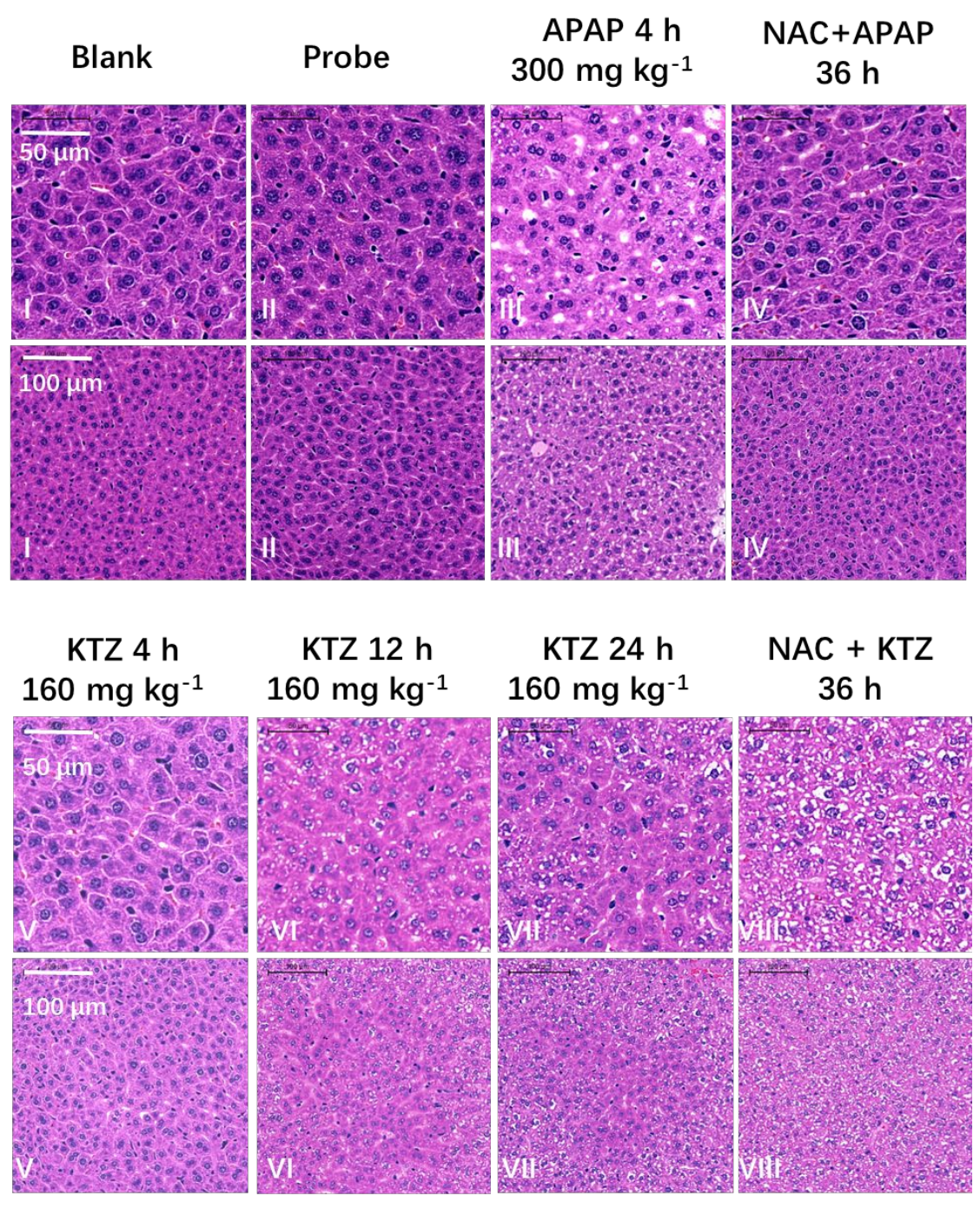

Figure S22. Typical H\&E staining images of the liver of mice treated under various conditions. (I) blank (the control group); (II) P-ONOO (4 h); (III) APAP (300 mg kg-1, $4 \mathrm{~h}$ ); (IV) NAC (300 mg kg-1) and APAP (300 mg kg-1, $36 \mathrm{~h})$; (V) KTZ (160 mg kg-1, $4 \mathrm{~h}$ ); (VI) KTZ (160 mg $\mathrm{kg}^{-1}, 12 \mathrm{~h}$ ); (VII) KTZ (160 mg kg-1, $\left.24 \mathrm{~h}\right)$; (VIII) NAC (300 mg kg-1) and KTZ (160 mg kg-1, $36 \mathrm{~h})$. 
MALDI-TOF mass spectroscopic characterization of related complexes

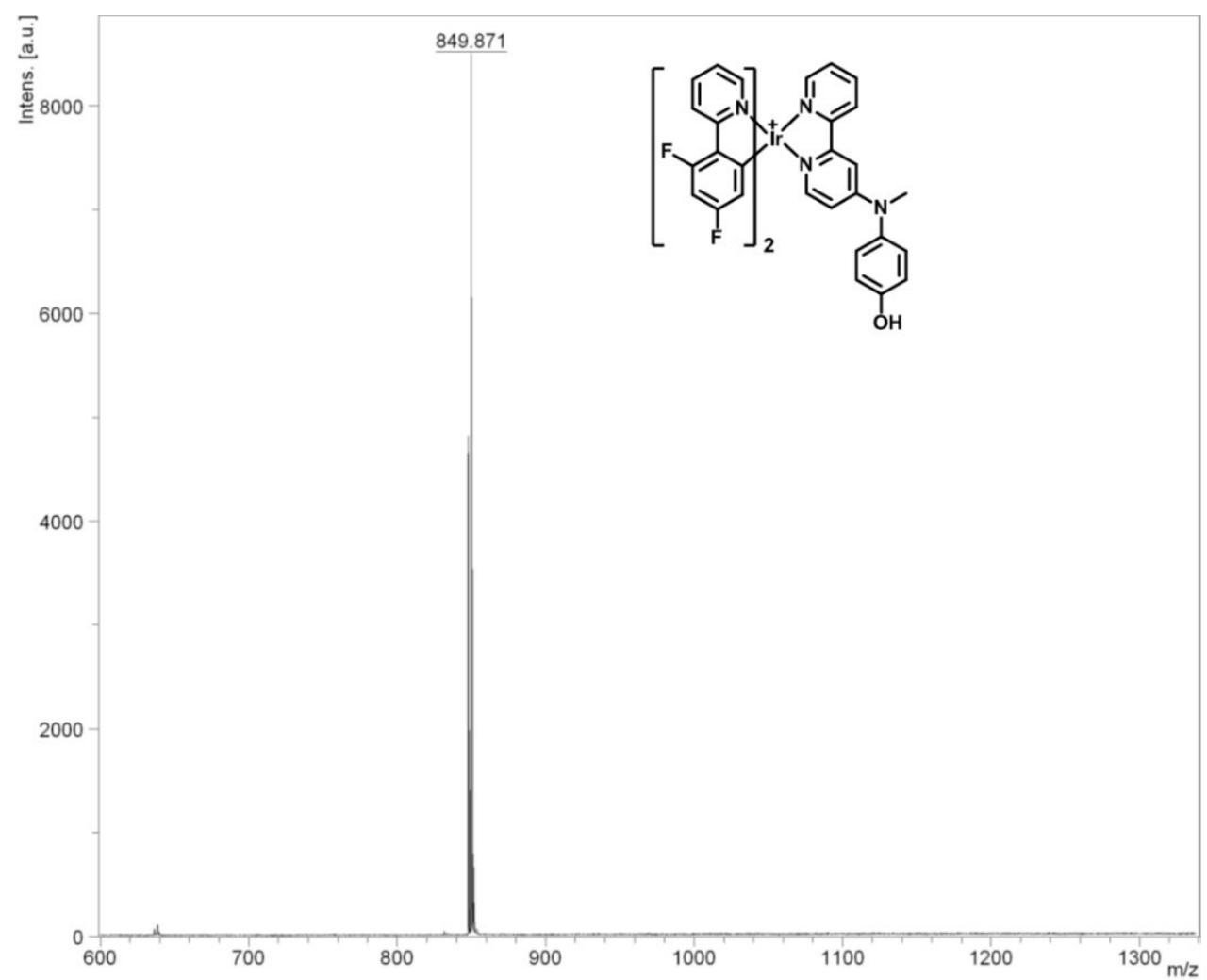

Figure S23. MALDI-TOF mass spectroscopic characterization of Ir1.

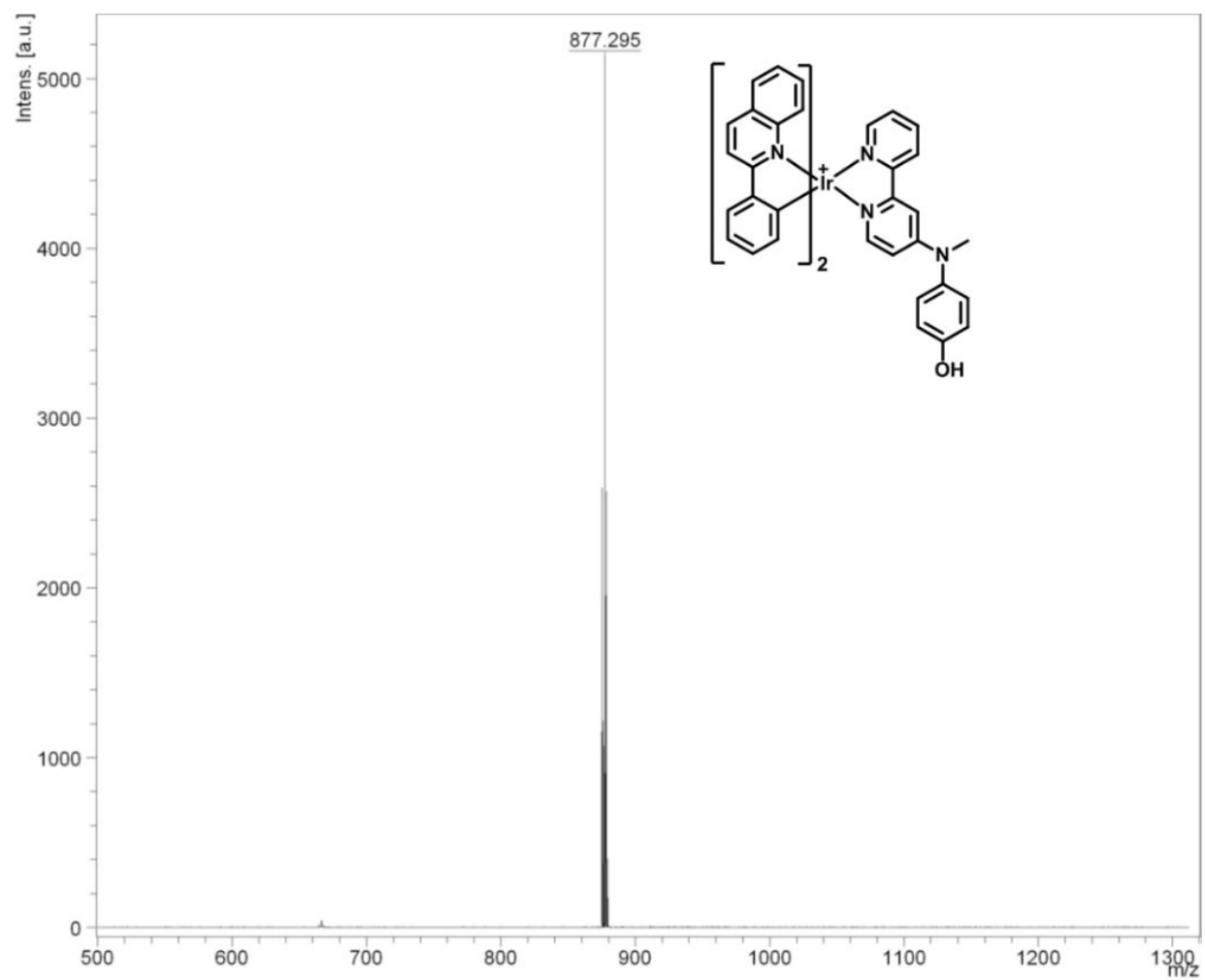

Figure S24. MALDI-TOF mass spectroscopic characterization of Ir2. 


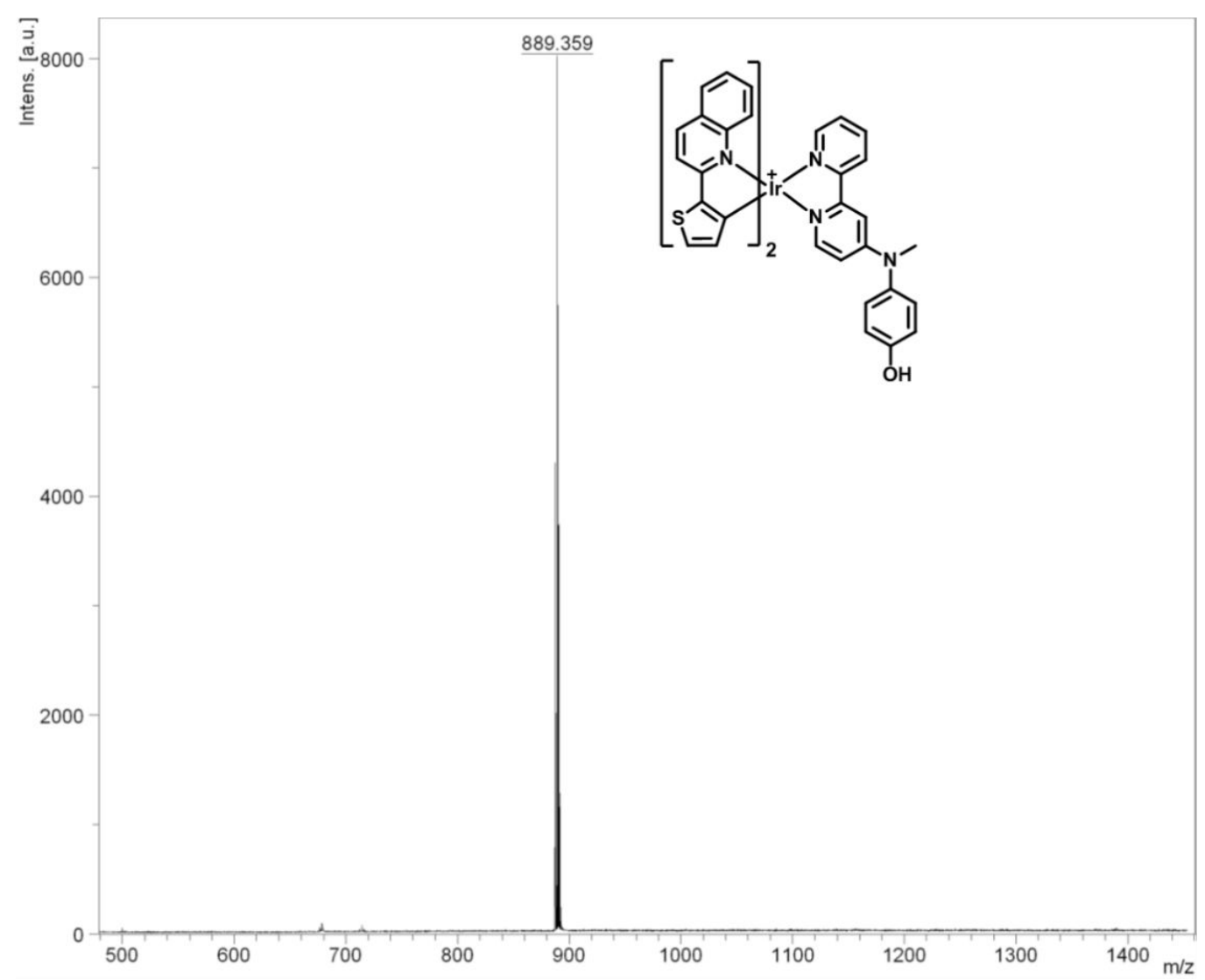

Figure S25. MALDI-TOF mass spectroscopic characterization of Ir3.

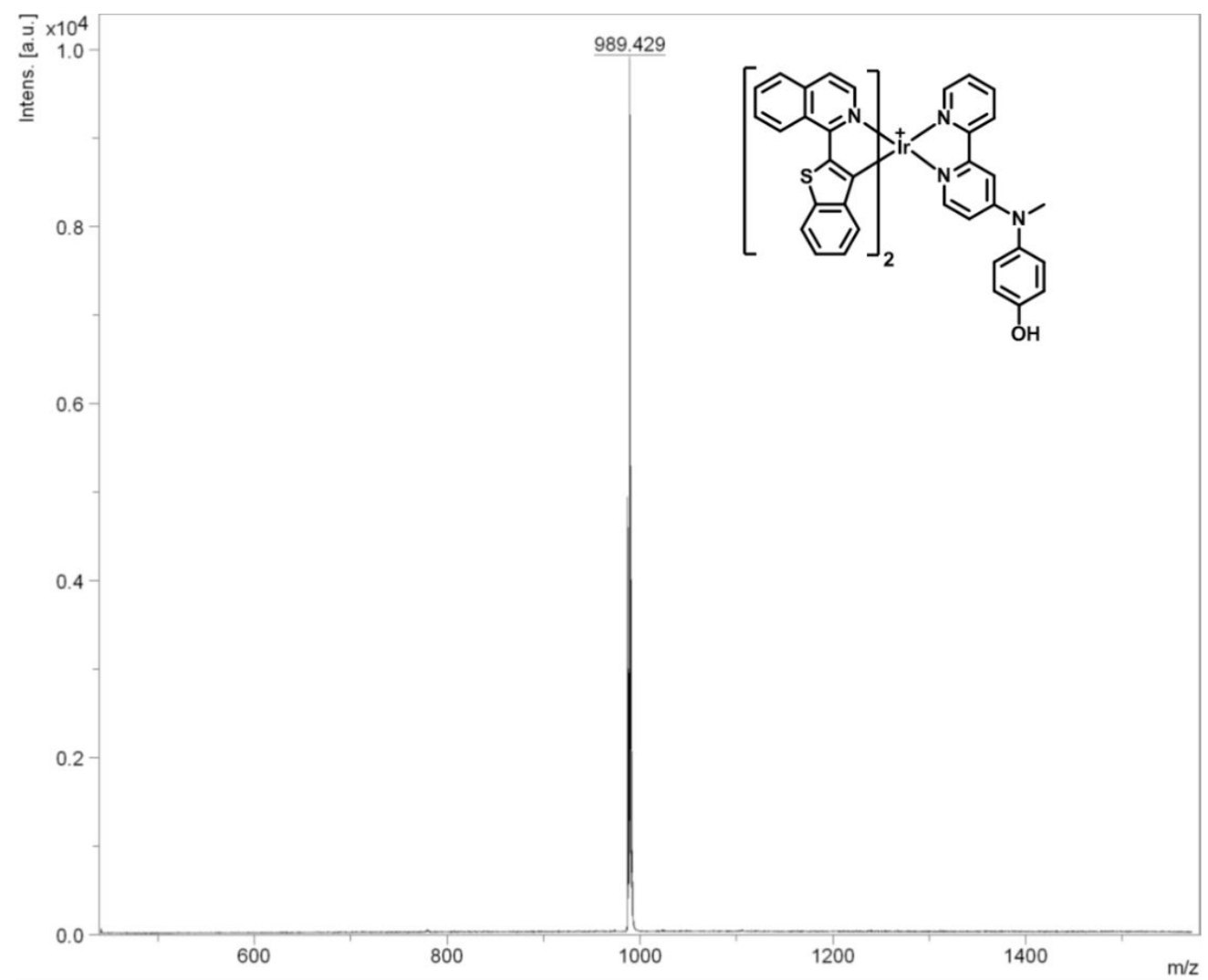

Figure S26. MALDI-TOF mass spectroscopic characterization of Ir4. 


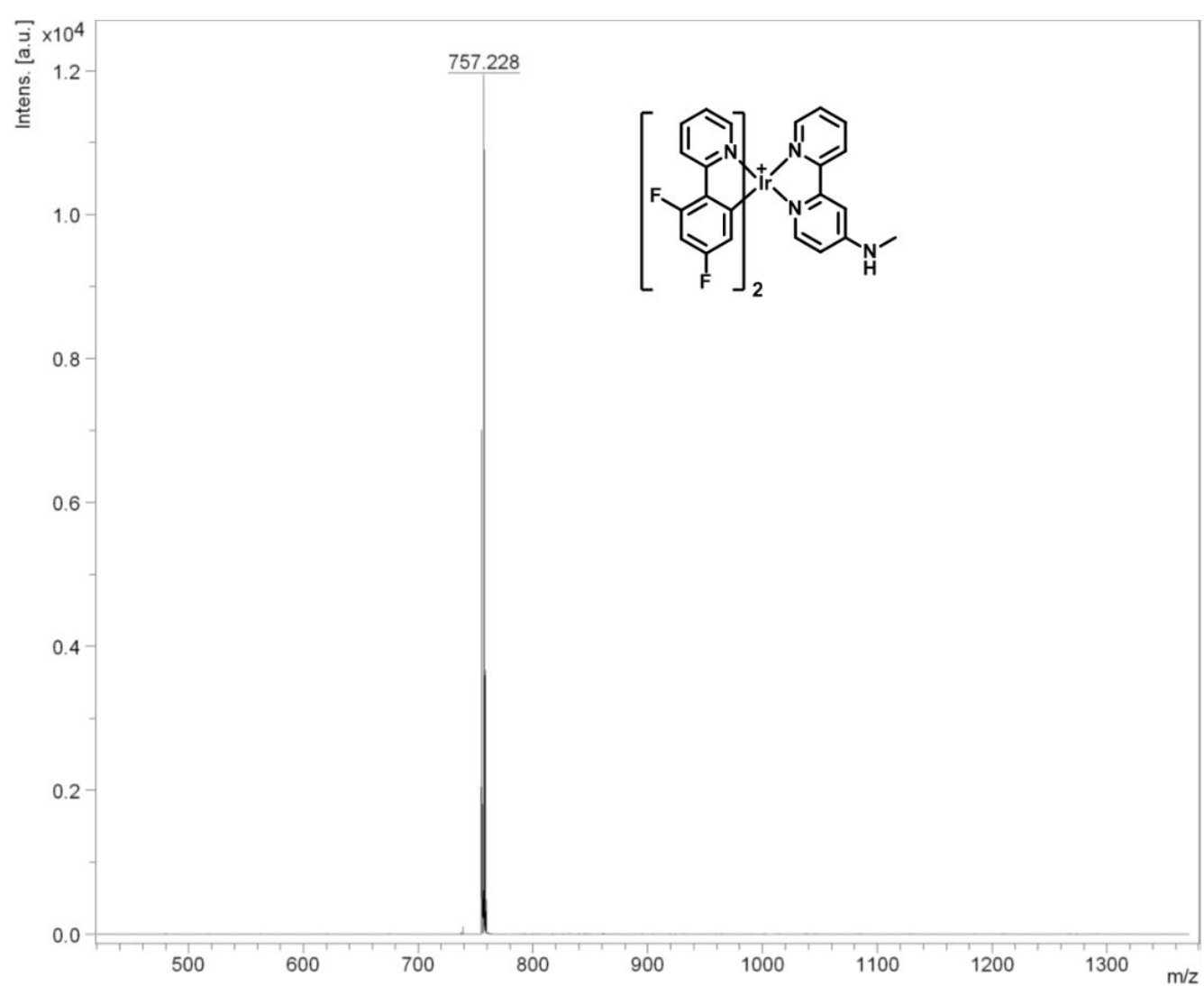

Figure S27. MALDI-TOF mass spectroscopic characterization of Ir1*.

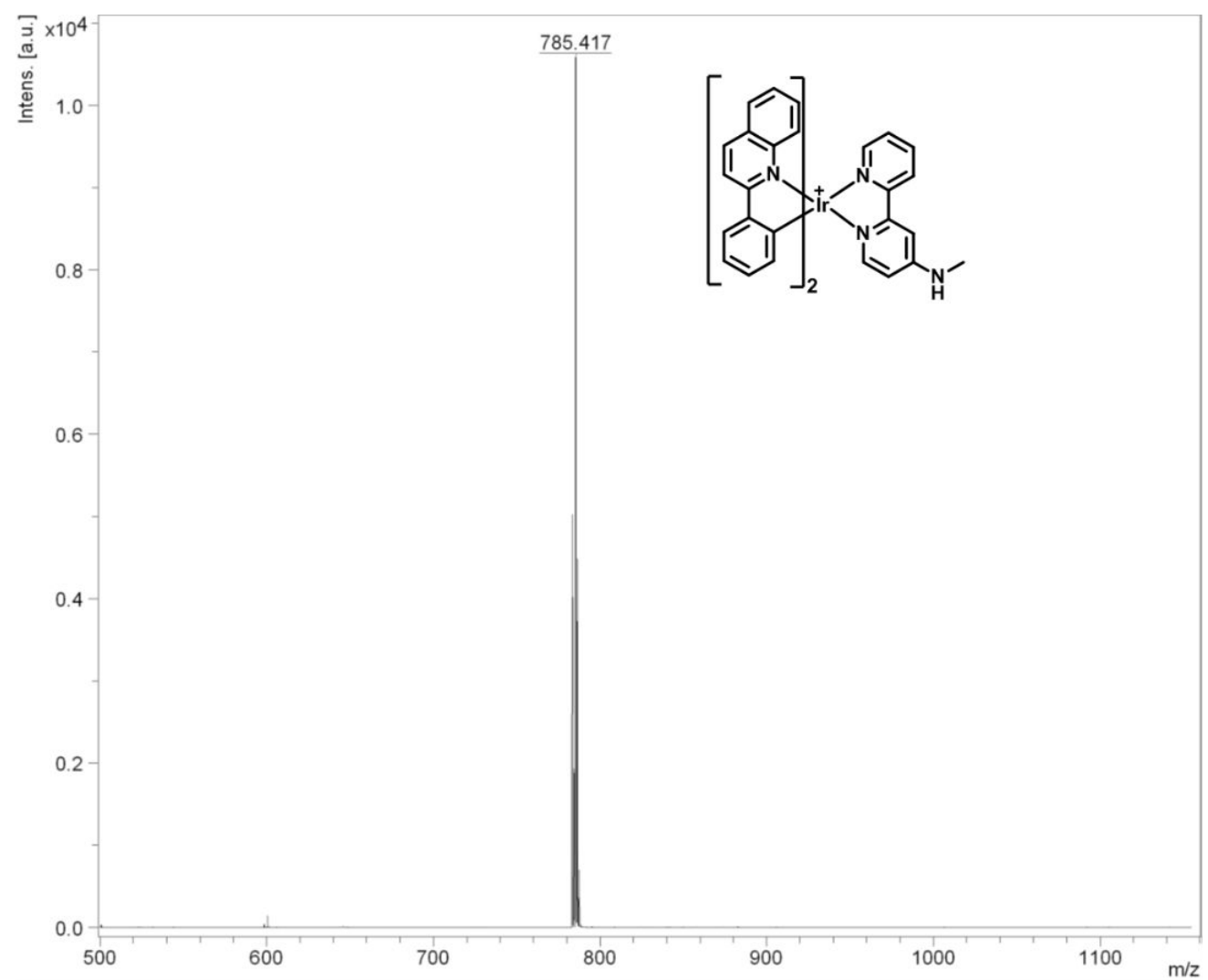

Figure S28. MALDI-TOF mass spectroscopic characterization of Ir2*. 


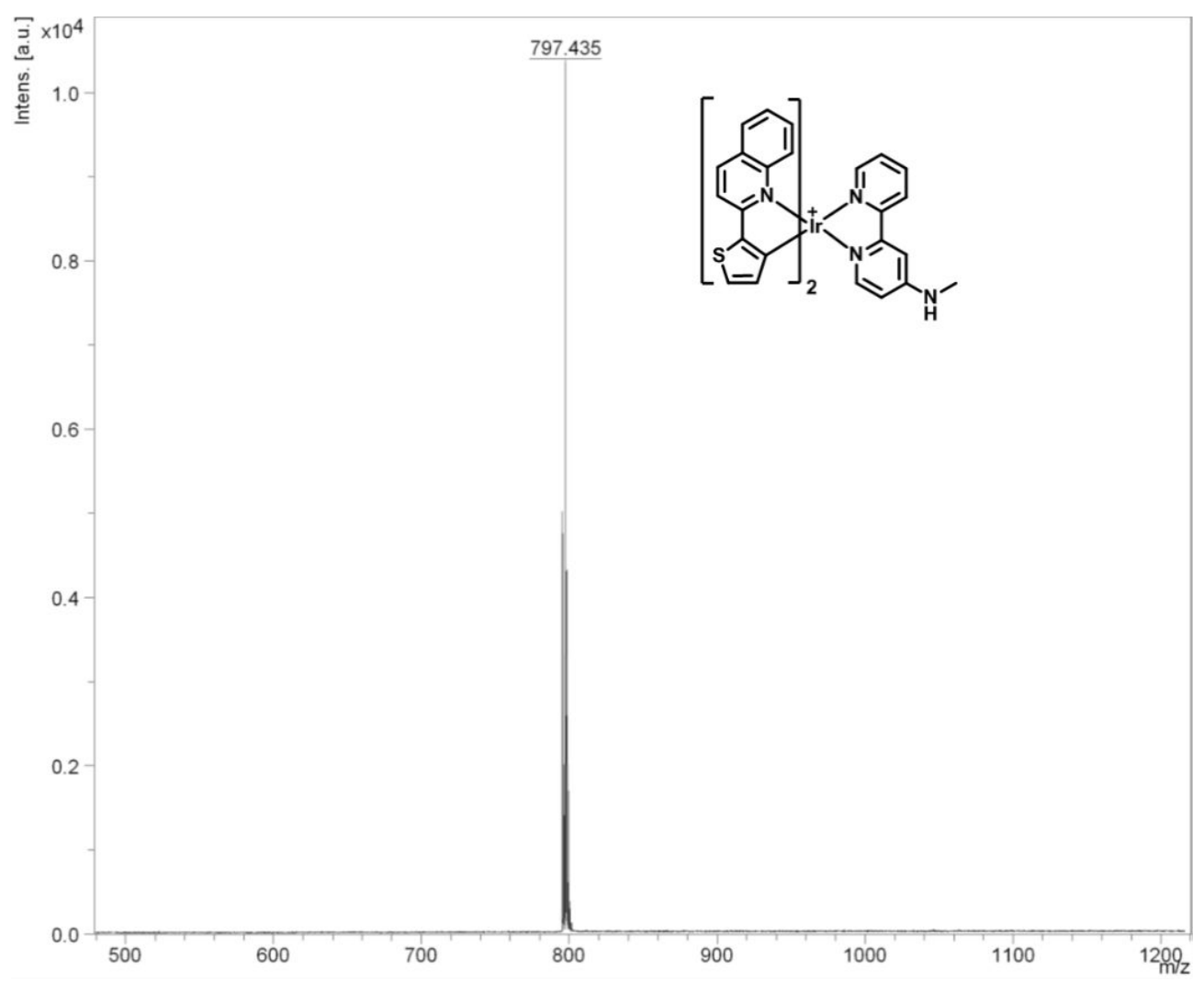

Figure S29. MALDI-TOF mass spectroscopic characterization of Ir3*.

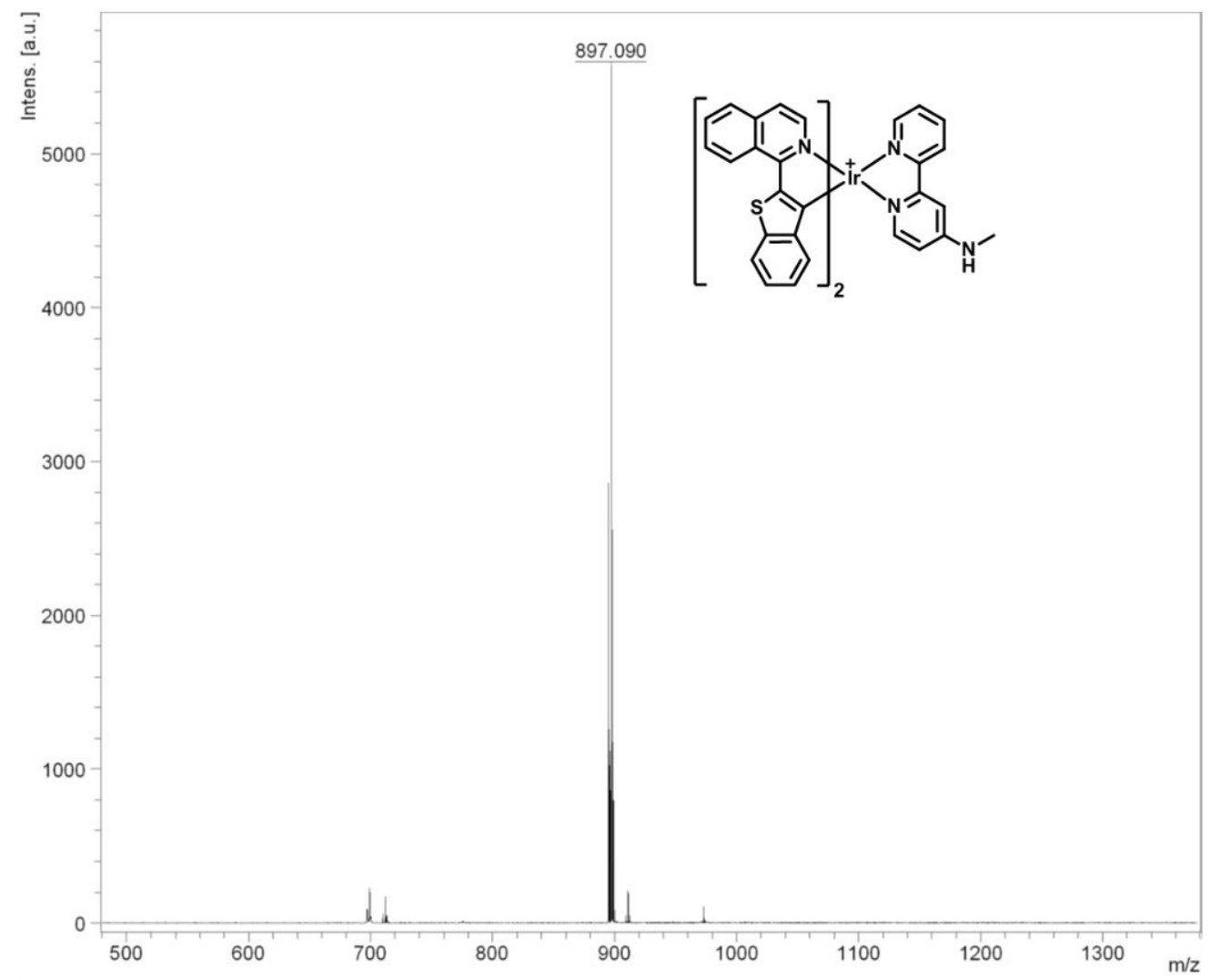

Figure S30. MALDI-TOF mass spectroscopic characterization of Ir4*. 


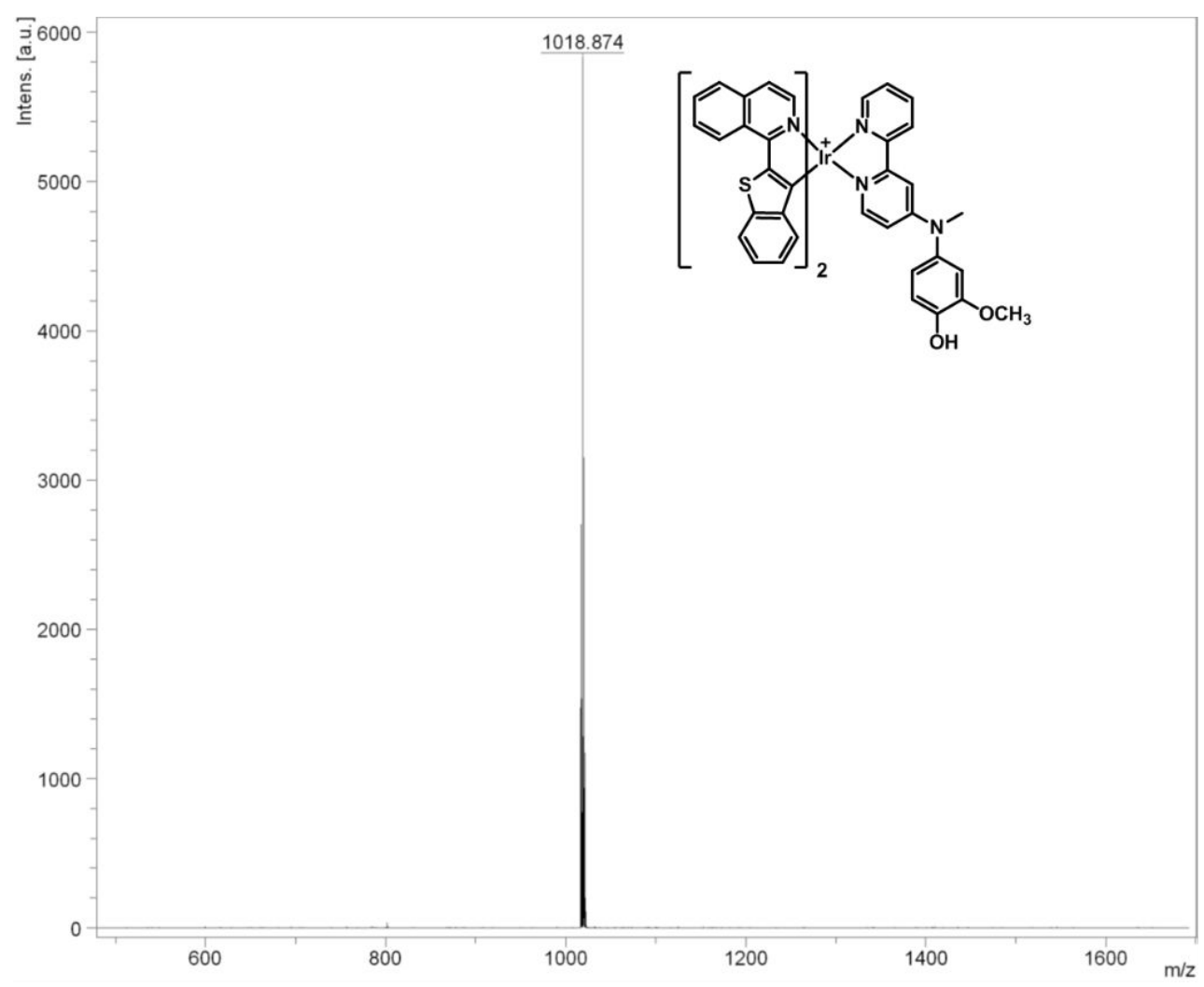

Figure S31. MALDI-TOF mass spectroscopic characterization of Ir5.

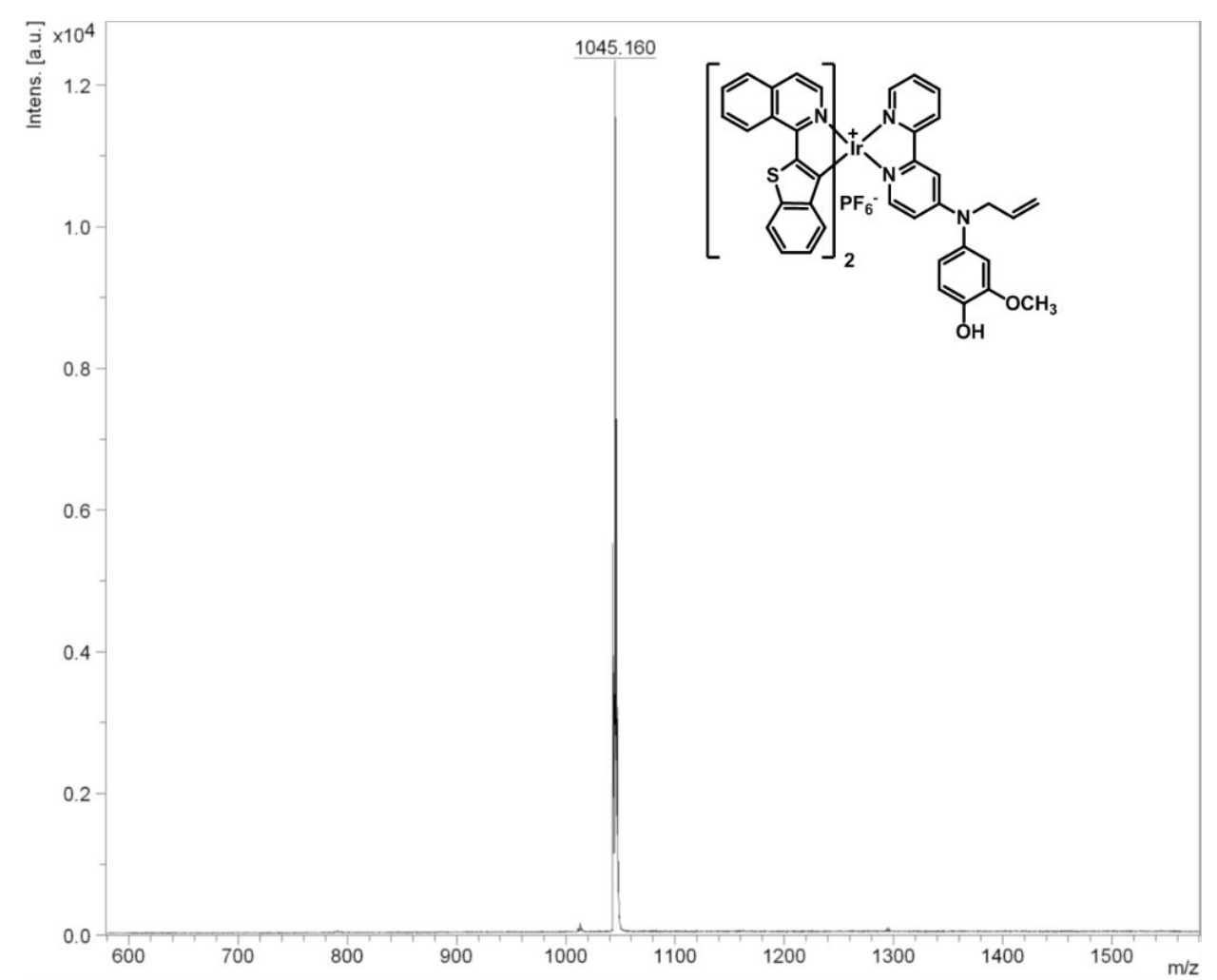

Figure S32. MALDI-TOF mass spectroscopic characterization of Ir6. 
NMR spectroscopic characterization of related complexes

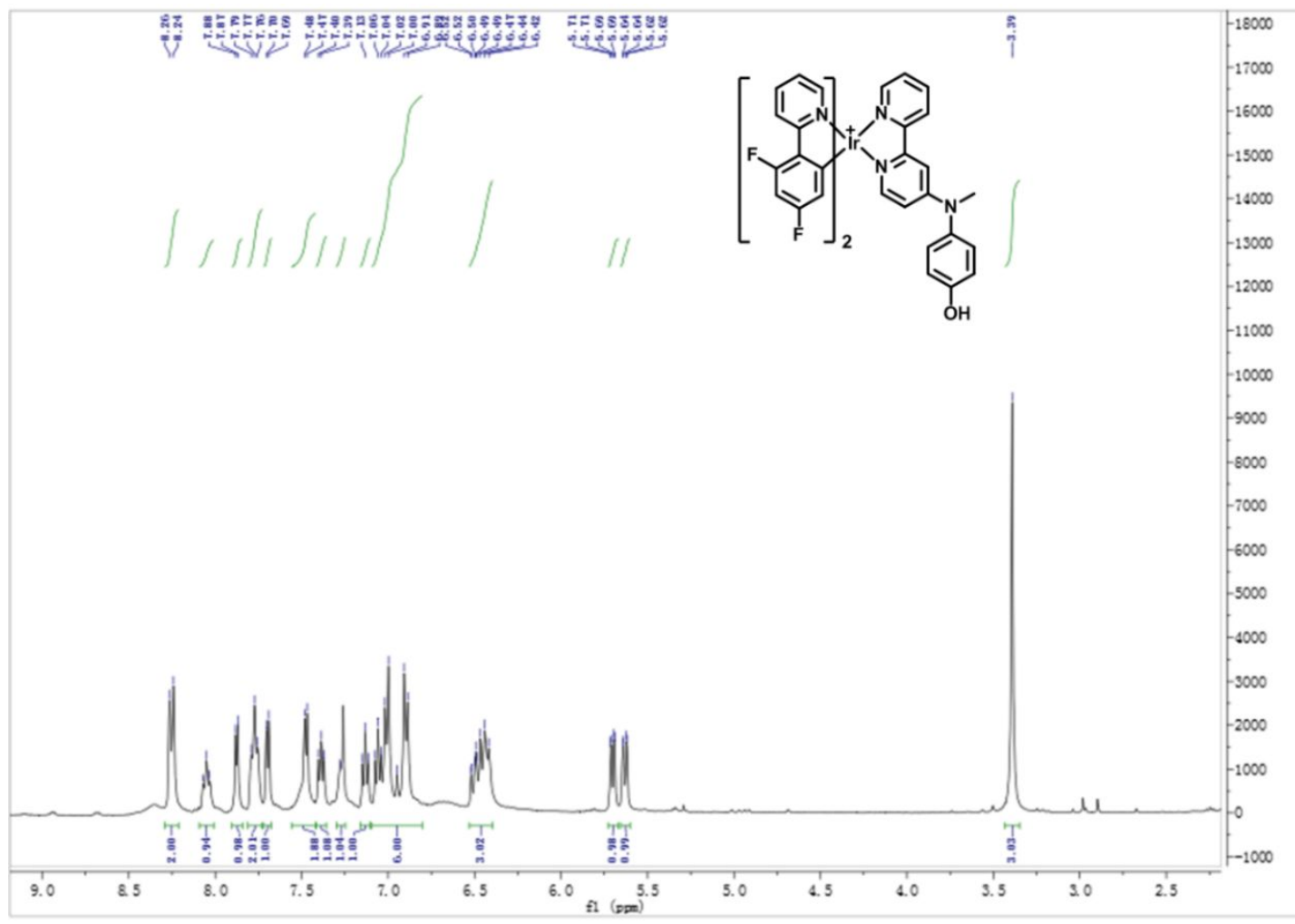

Figure S33. ${ }^{1} \mathrm{H}$ NMR spectroscopic characterization of Ir1.

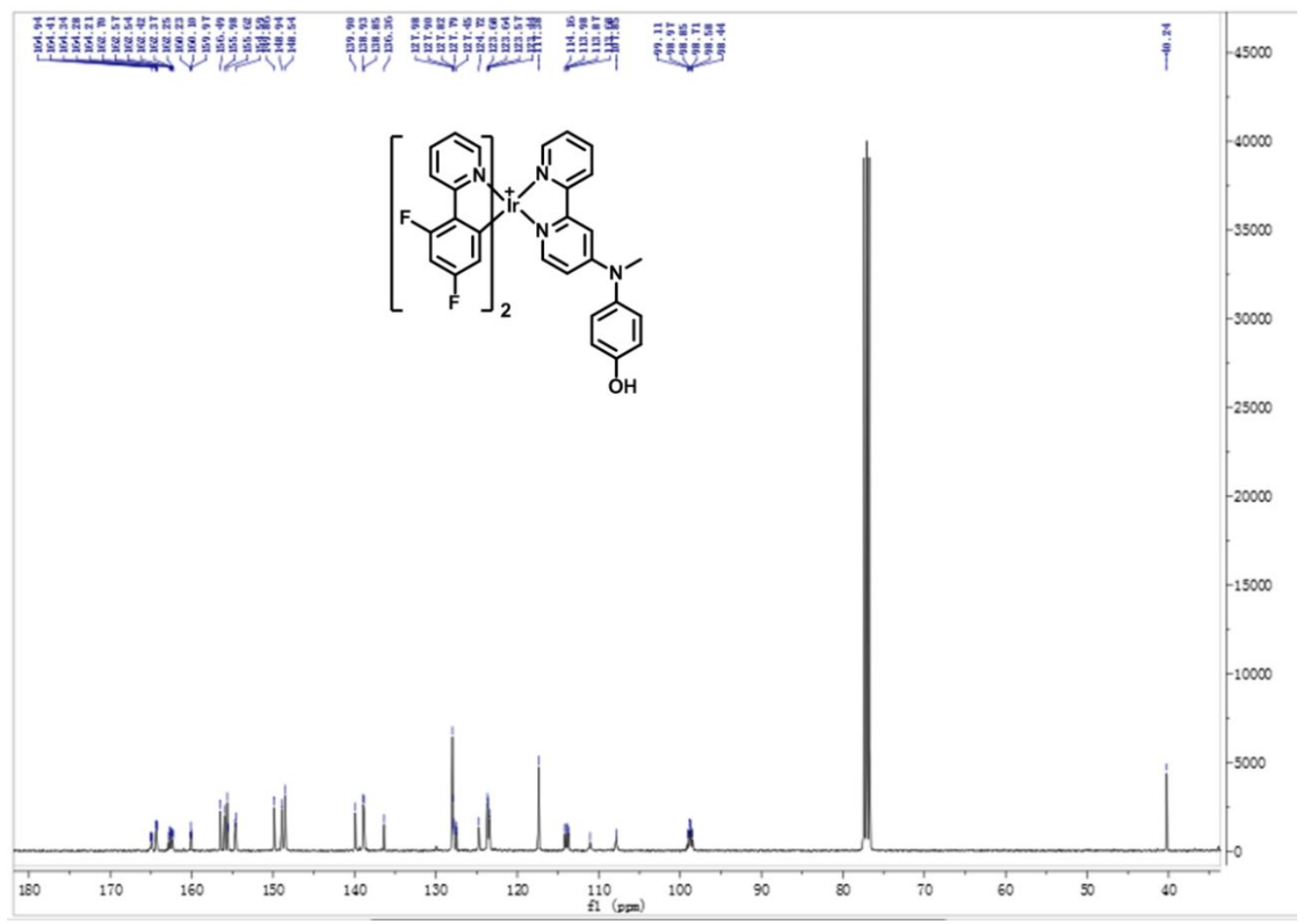

Figure S34. ${ }^{13} \mathrm{C}$ NMR spectroscopic characterization of Ir1. 


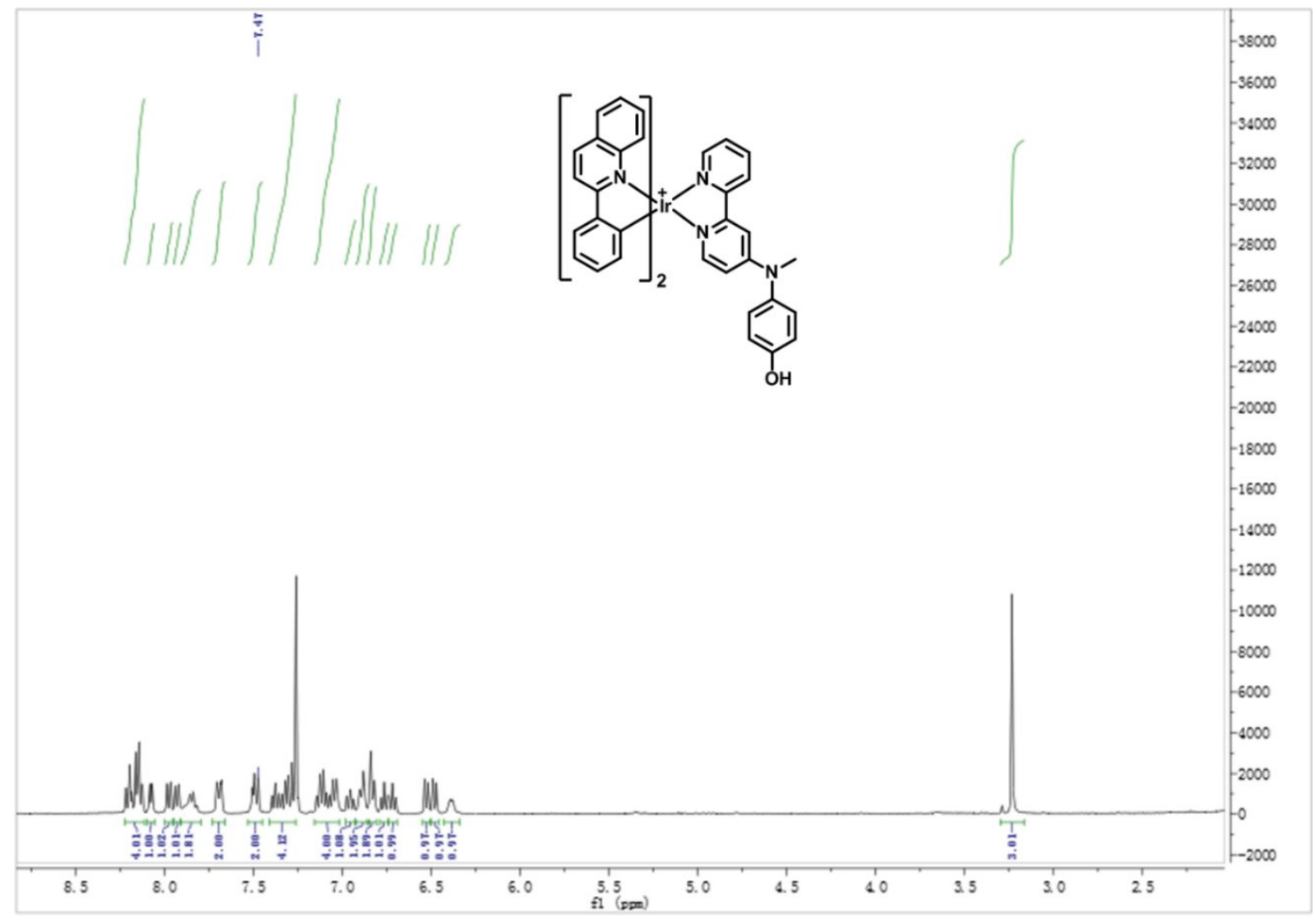

Figure S35. ${ }^{1} \mathrm{H}$ NMR spectroscopic characterization of Ir2.

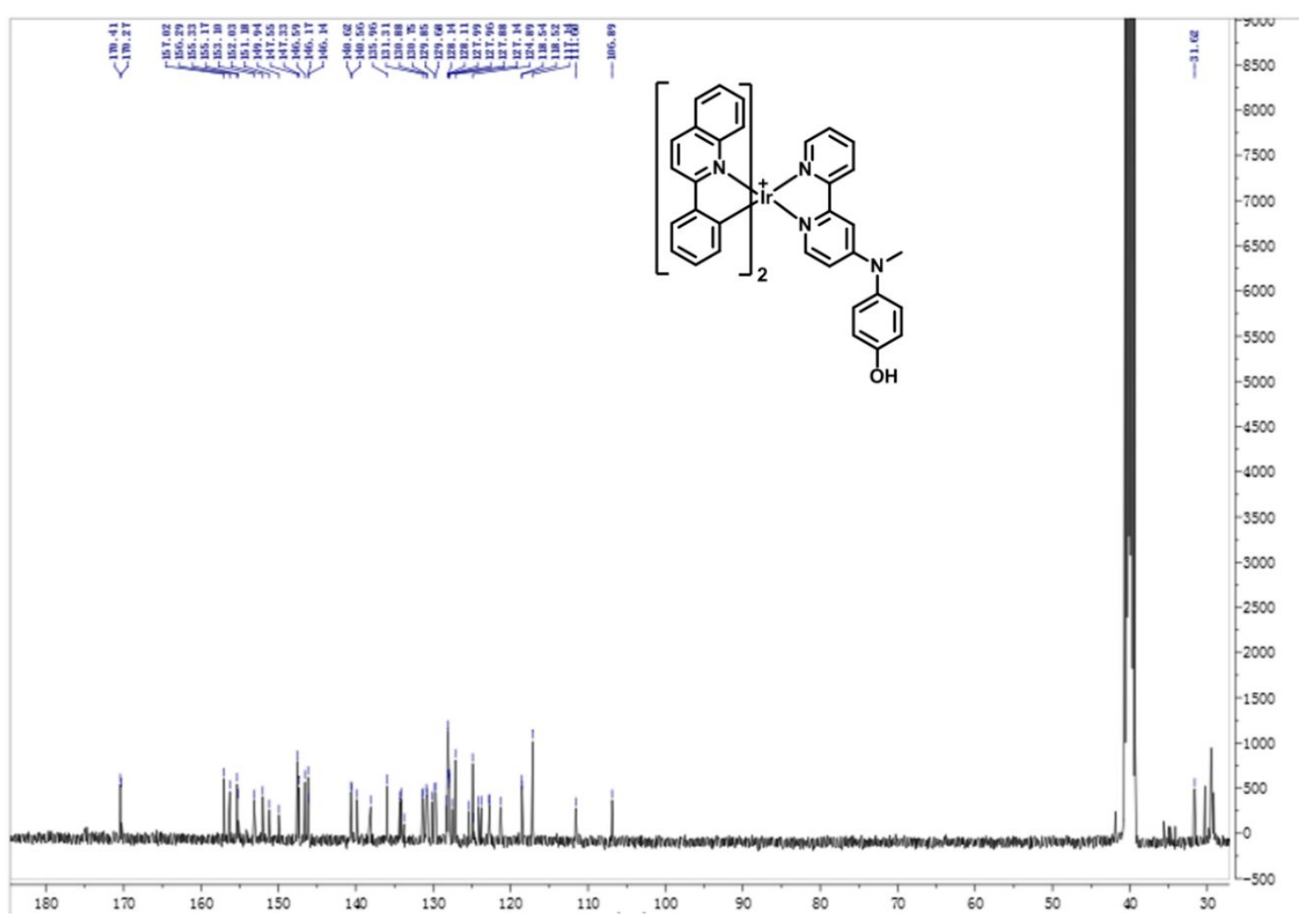

Figure S36. ${ }^{13} \mathrm{C}$ NMR spectroscopic characterization of Ir2. 


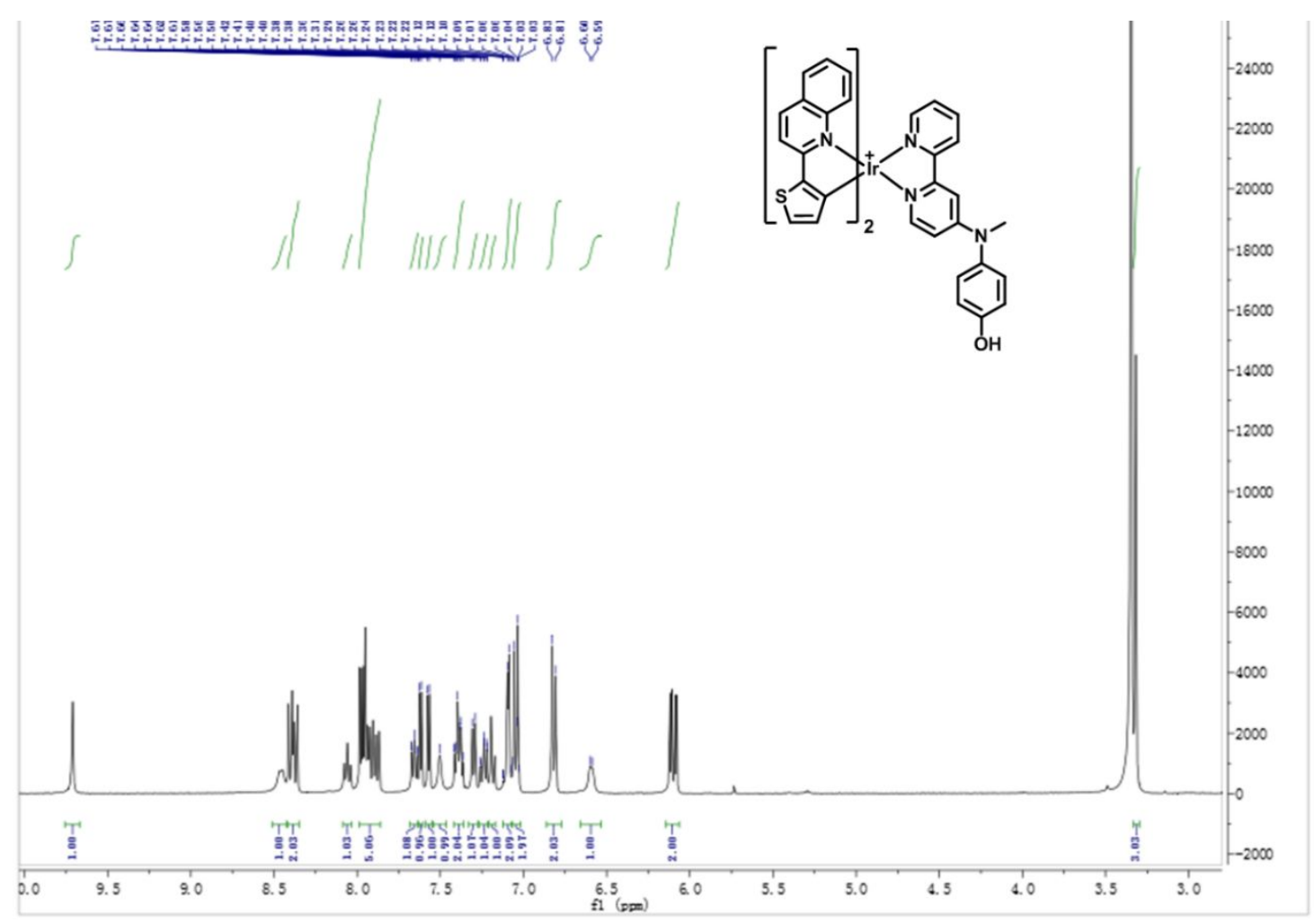

Figure S37. ${ }^{1} \mathrm{H}$ NMR spectroscopic characterization of Ir3.

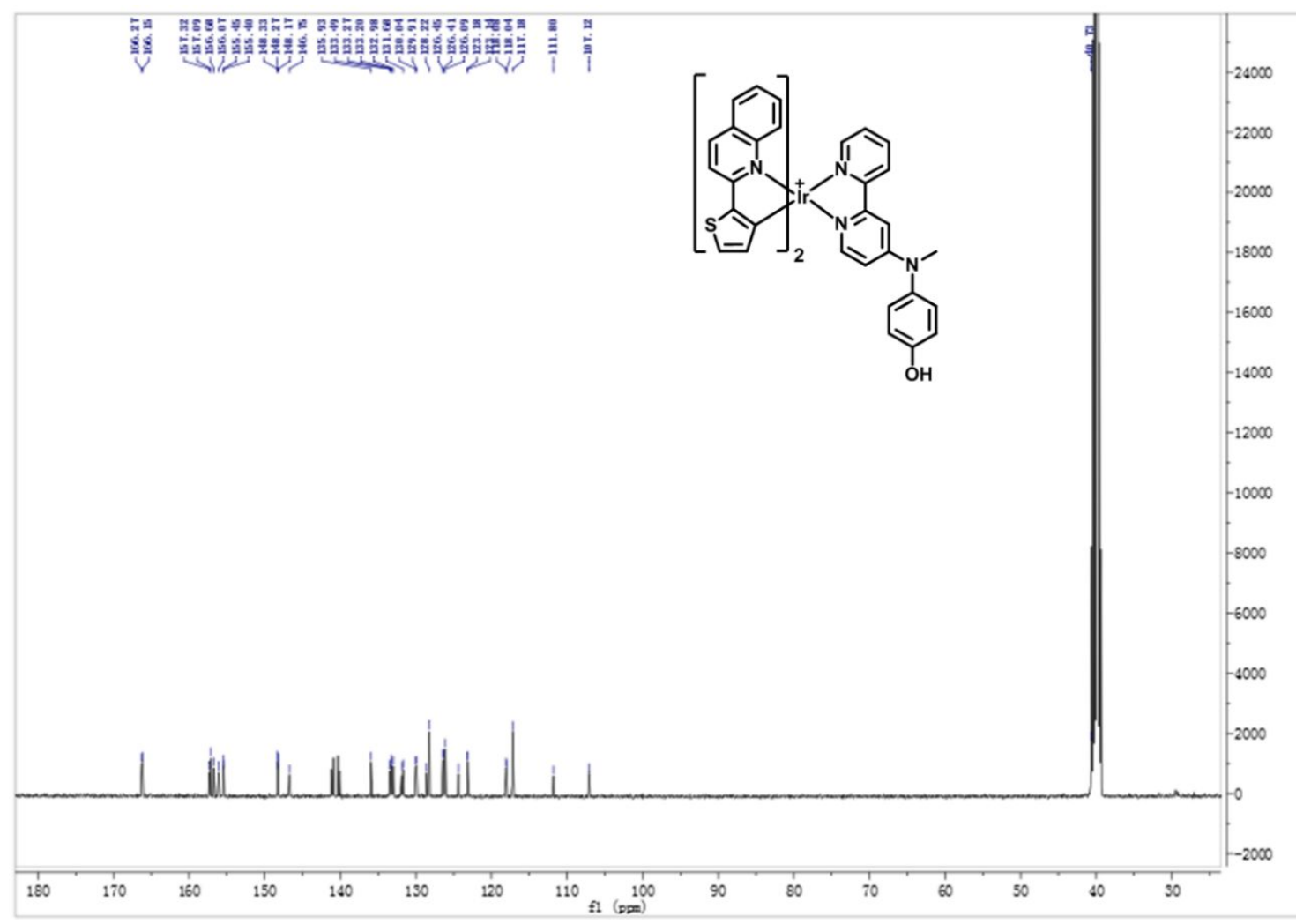

Figure S38. ${ }^{13} \mathrm{C}$ NMR spectroscopic characterization of Ir3. 


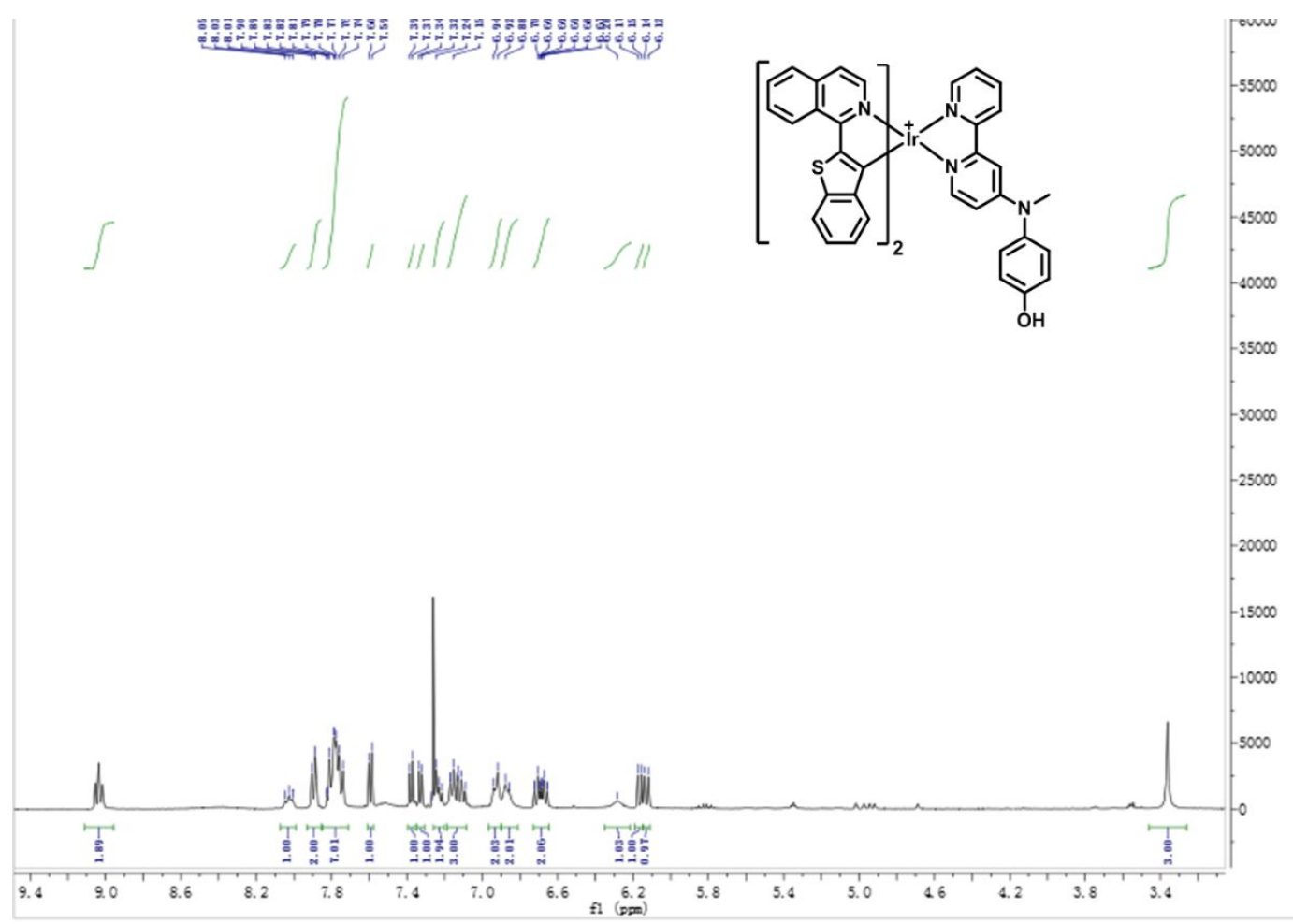

Figure S39. ${ }^{1} \mathrm{H}$ NMR spectroscopic characterization of Ir4.

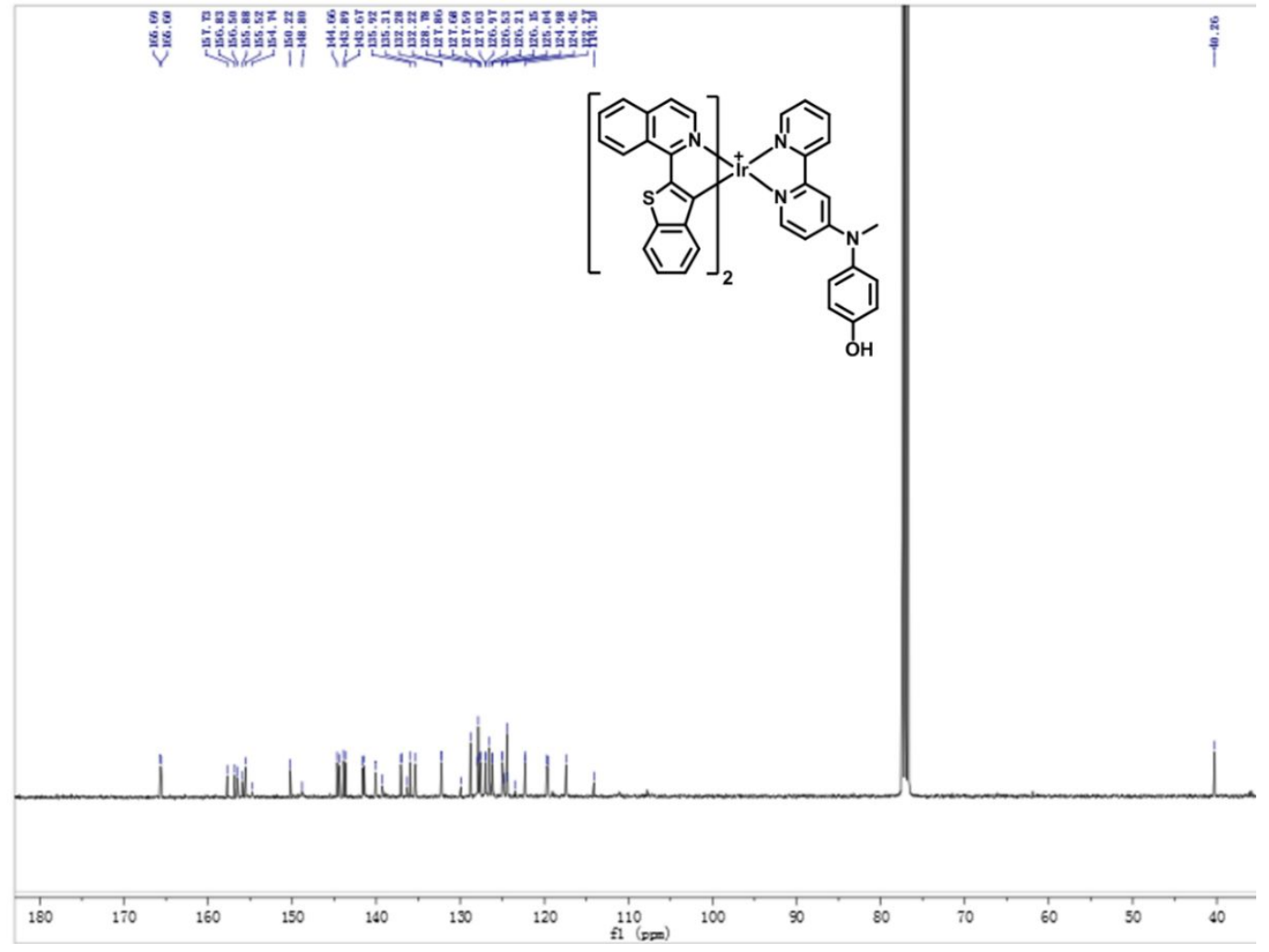

Figure S40. ${ }^{13} \mathrm{C}$ NMR spectroscopic characterization of Ir4. 


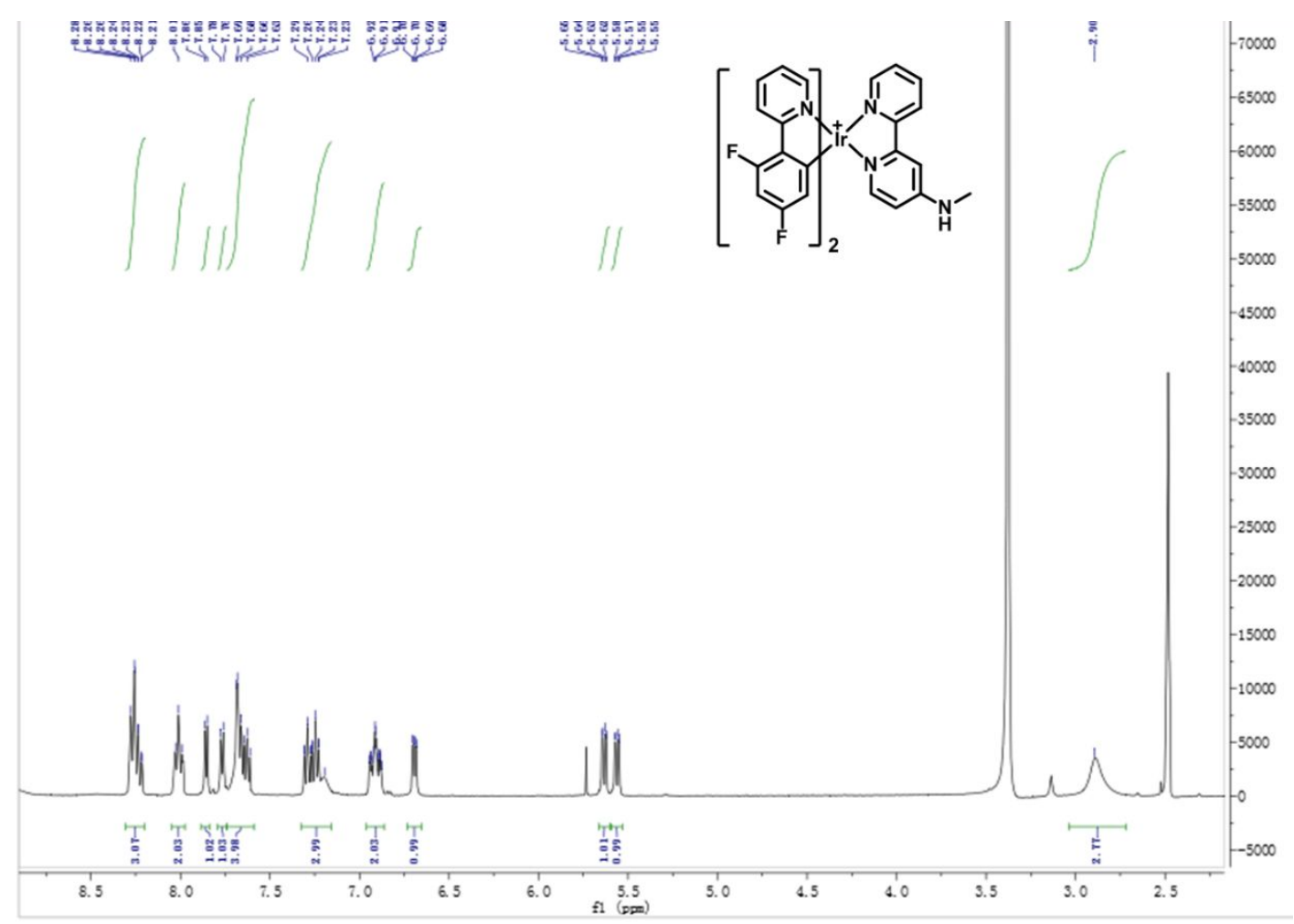

Figure S41. ${ }^{1} \mathrm{H}$ NMR spectroscopic characterization of Ir1*.

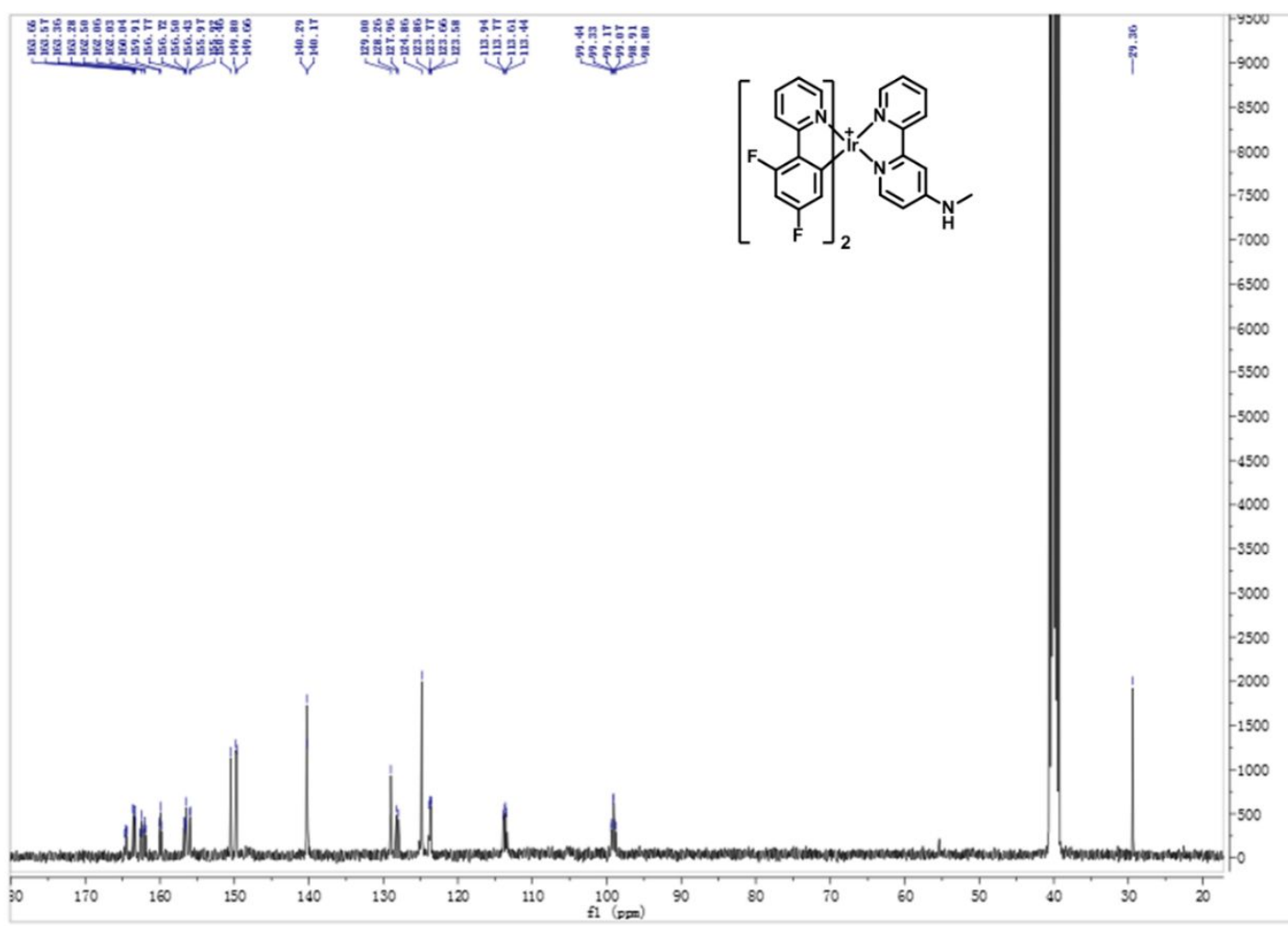

Figure S42. ${ }^{13} \mathrm{C}$ NMR spectroscopic characterization of Ir1*. 


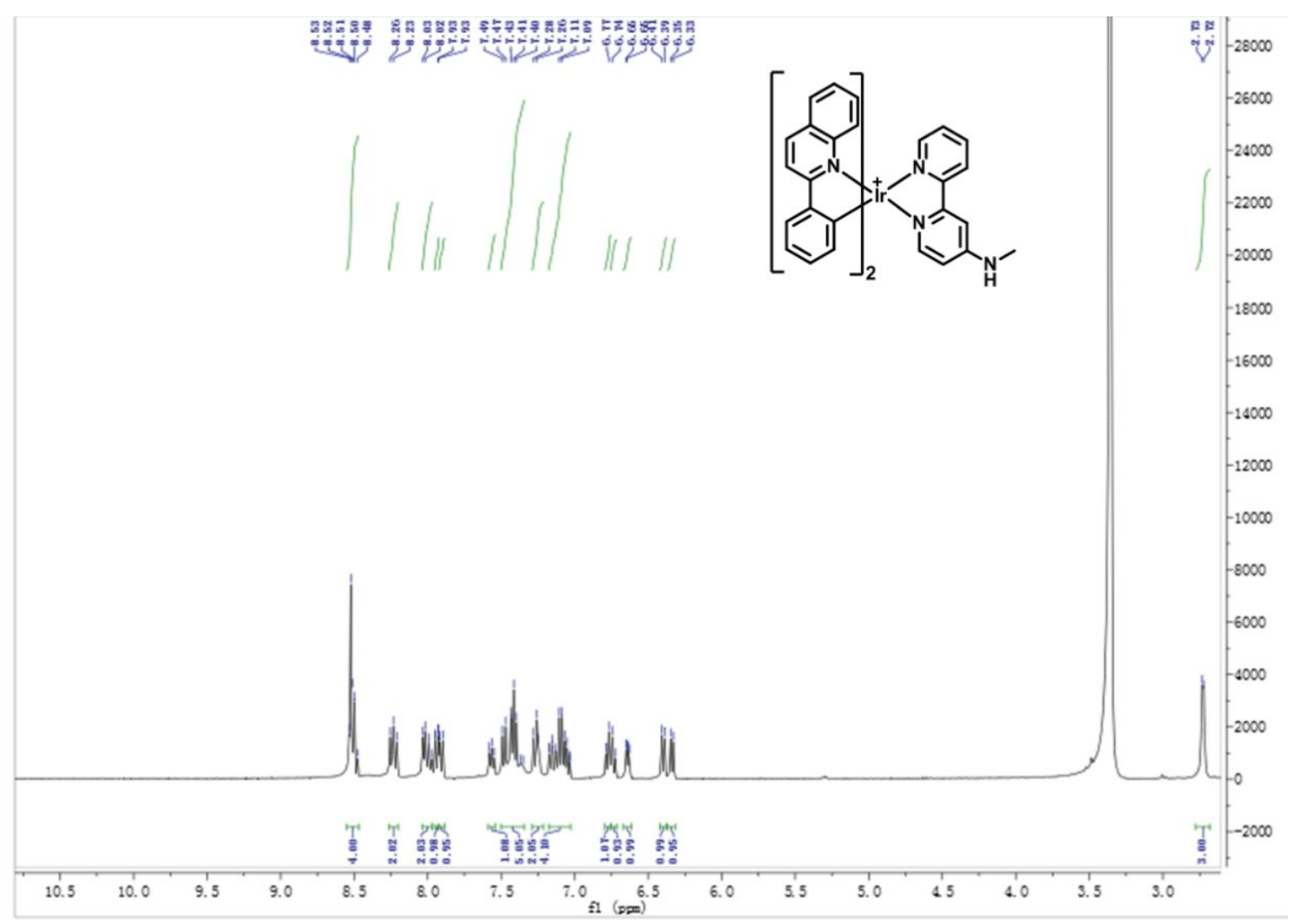

Figure S43. ${ }^{1} \mathrm{H}$ NMR spectroscopic characterization of Ir 2*.

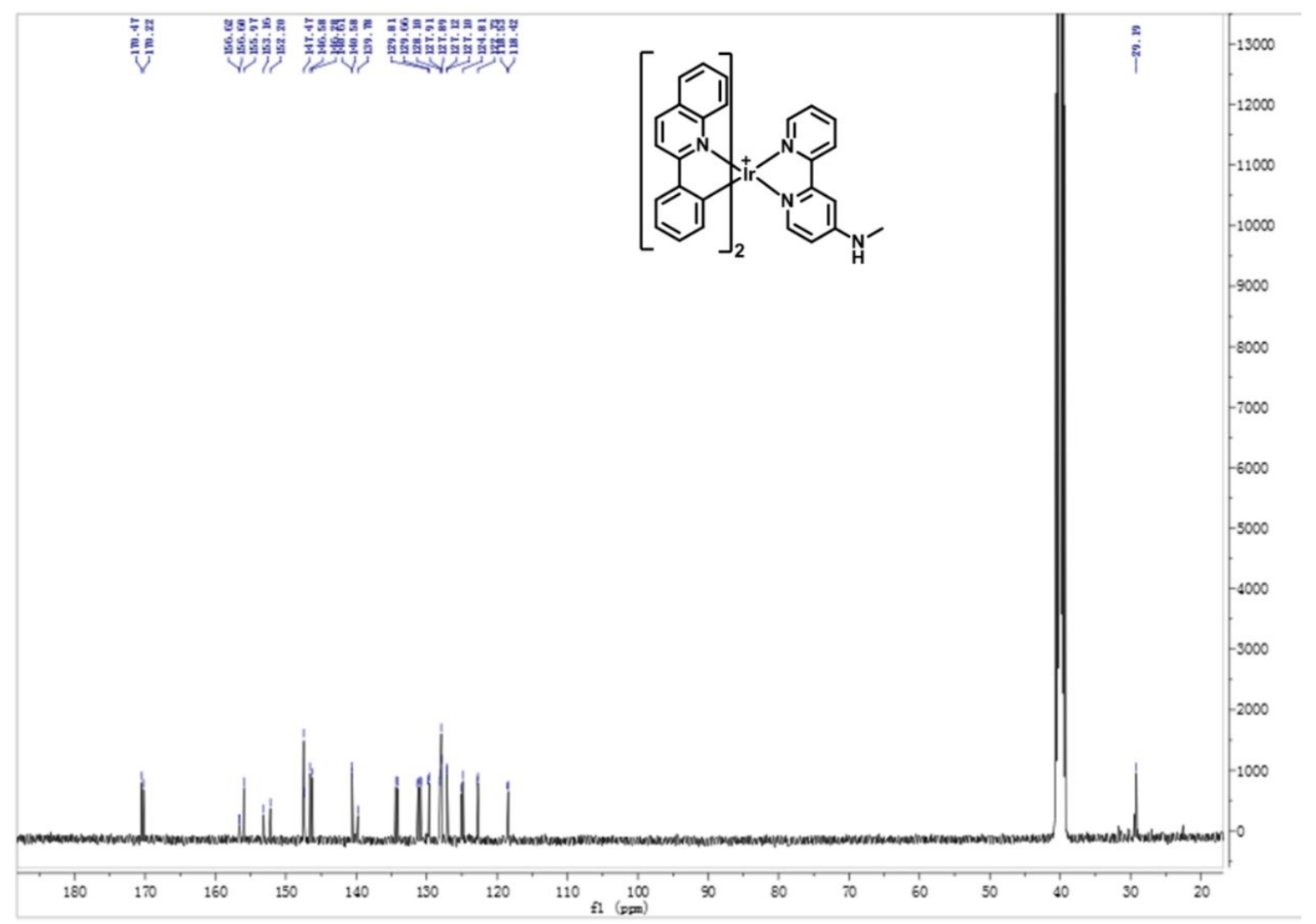

Figure S44. ${ }^{13} \mathrm{C}$ NMR spectroscopic characterization of Ir2*. 


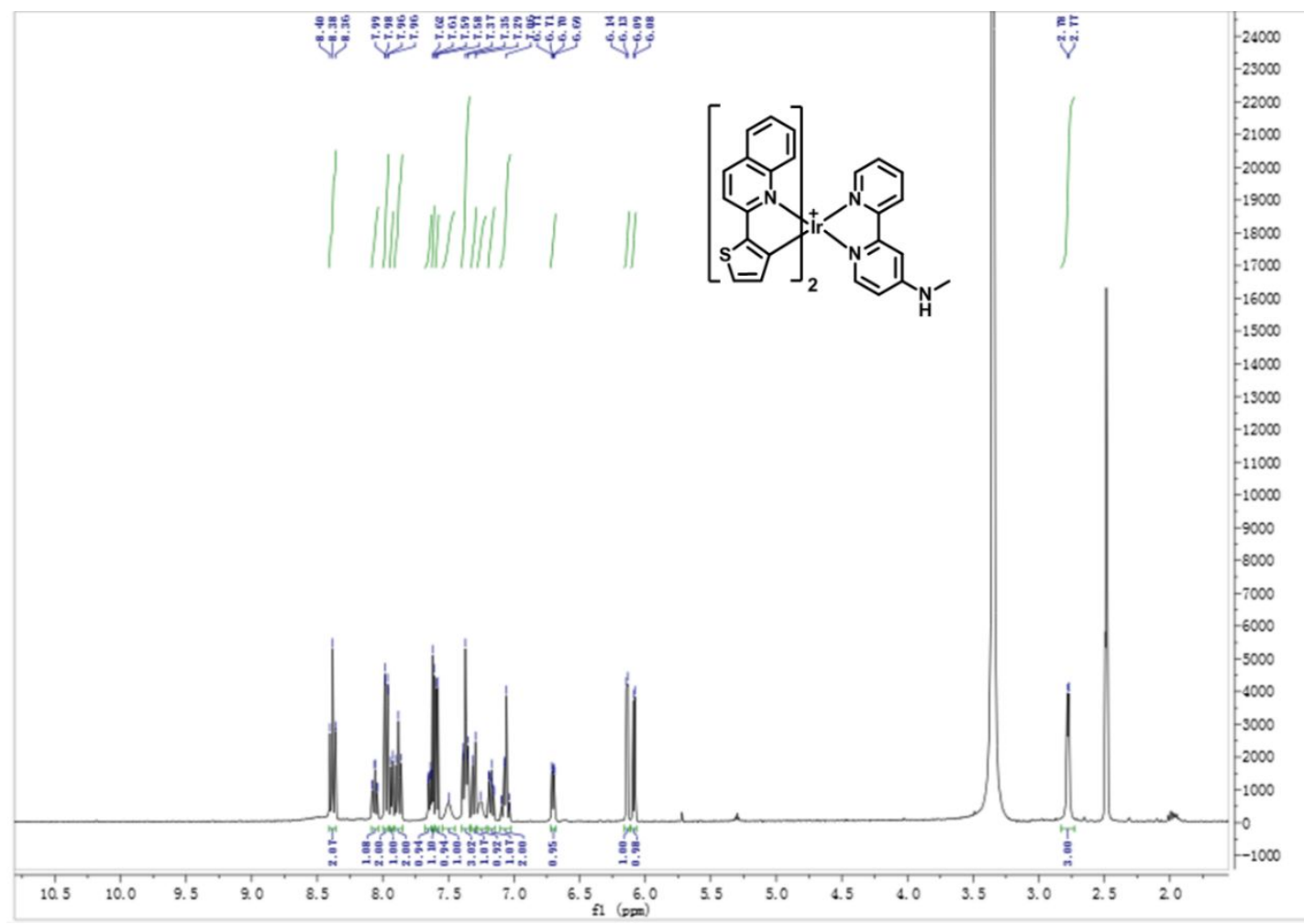

Figure S45. ${ }^{1} \mathrm{H}$ NMR spectroscopic characterization of Ir3*.

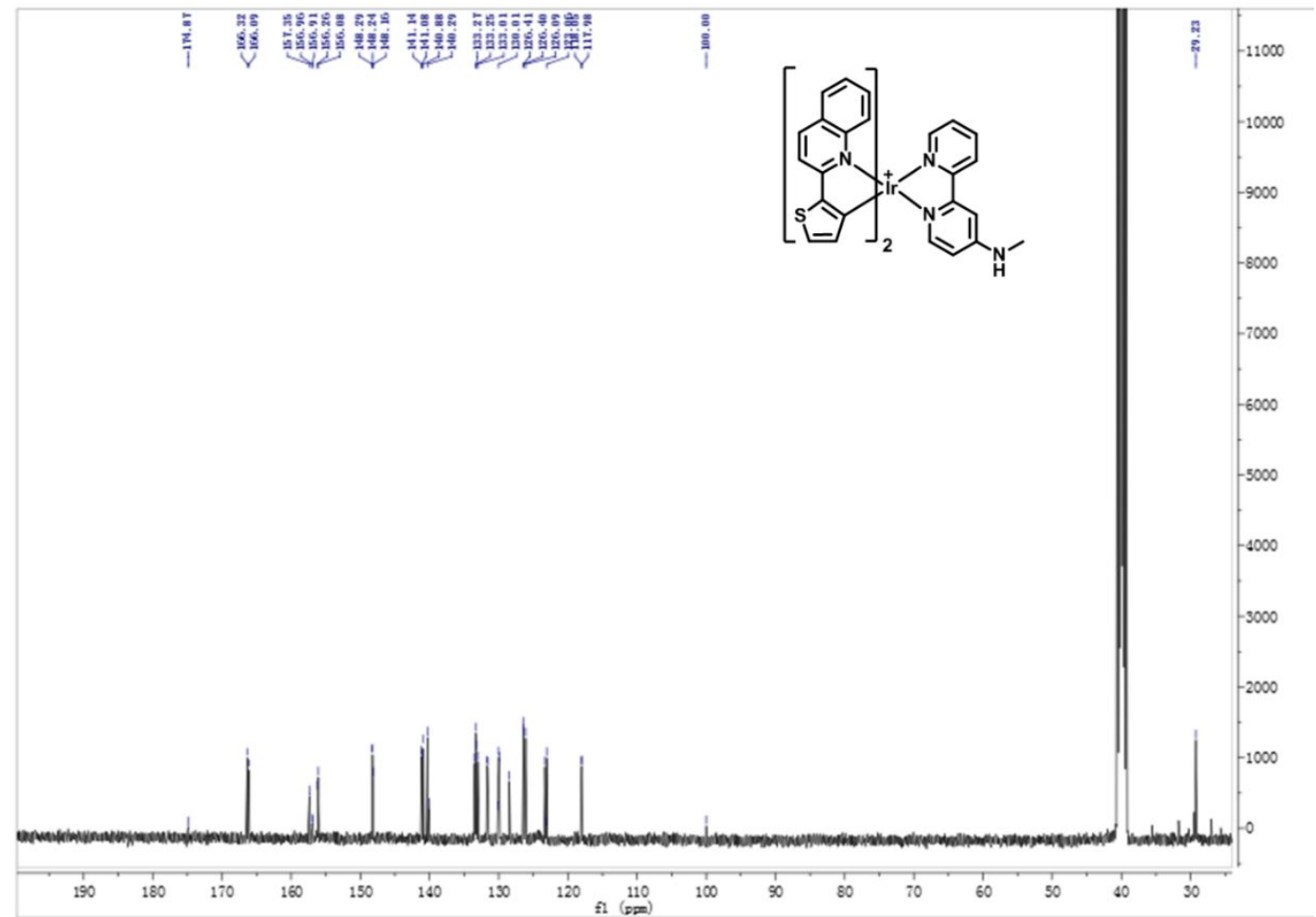

Figure S46. ${ }^{13} \mathrm{C}$ NMR spectroscopic characterization of Ir3*. 


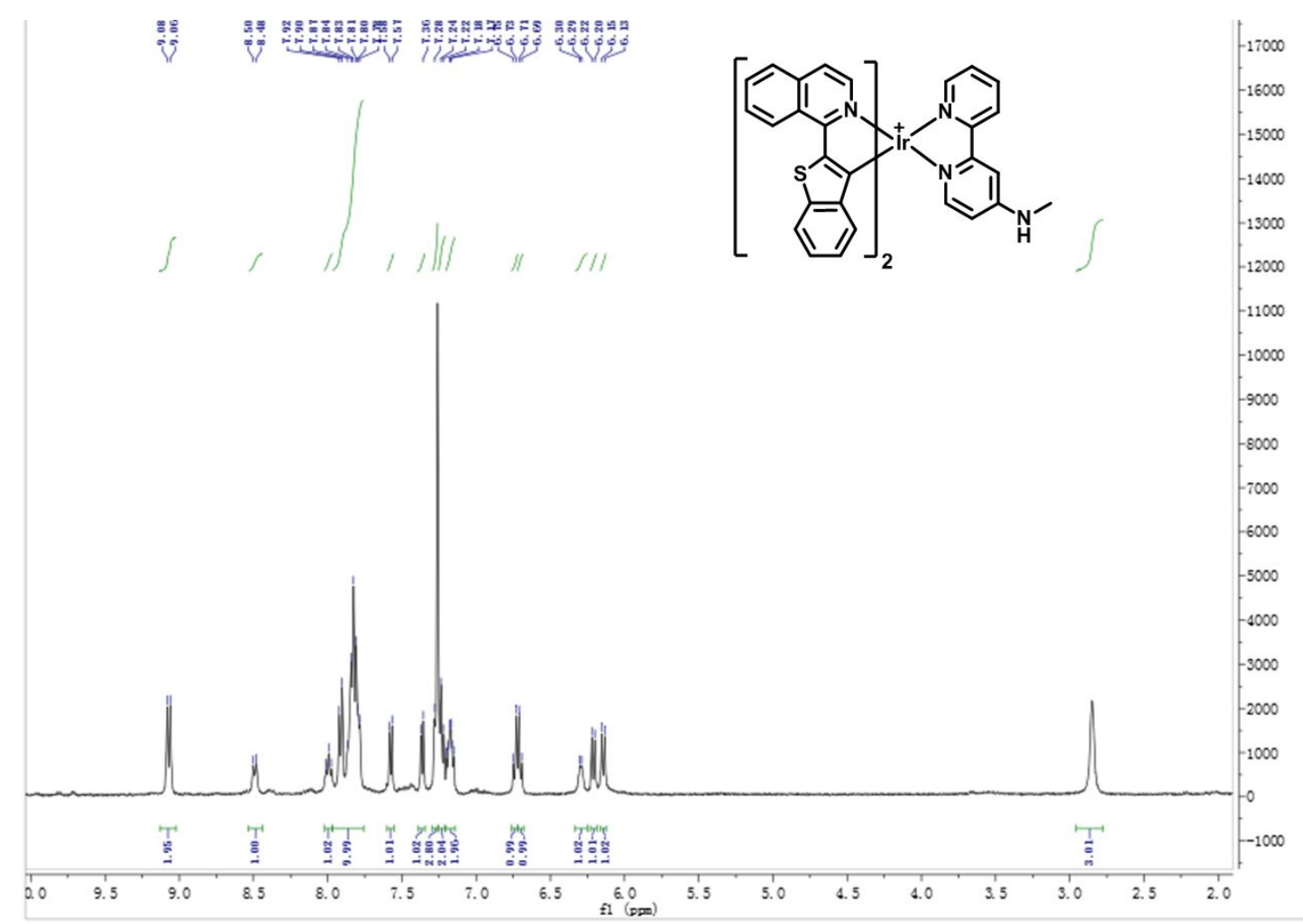

Figure S47. ${ }^{1} \mathrm{H}$ NMR spectroscopic characterization of Ir4*.

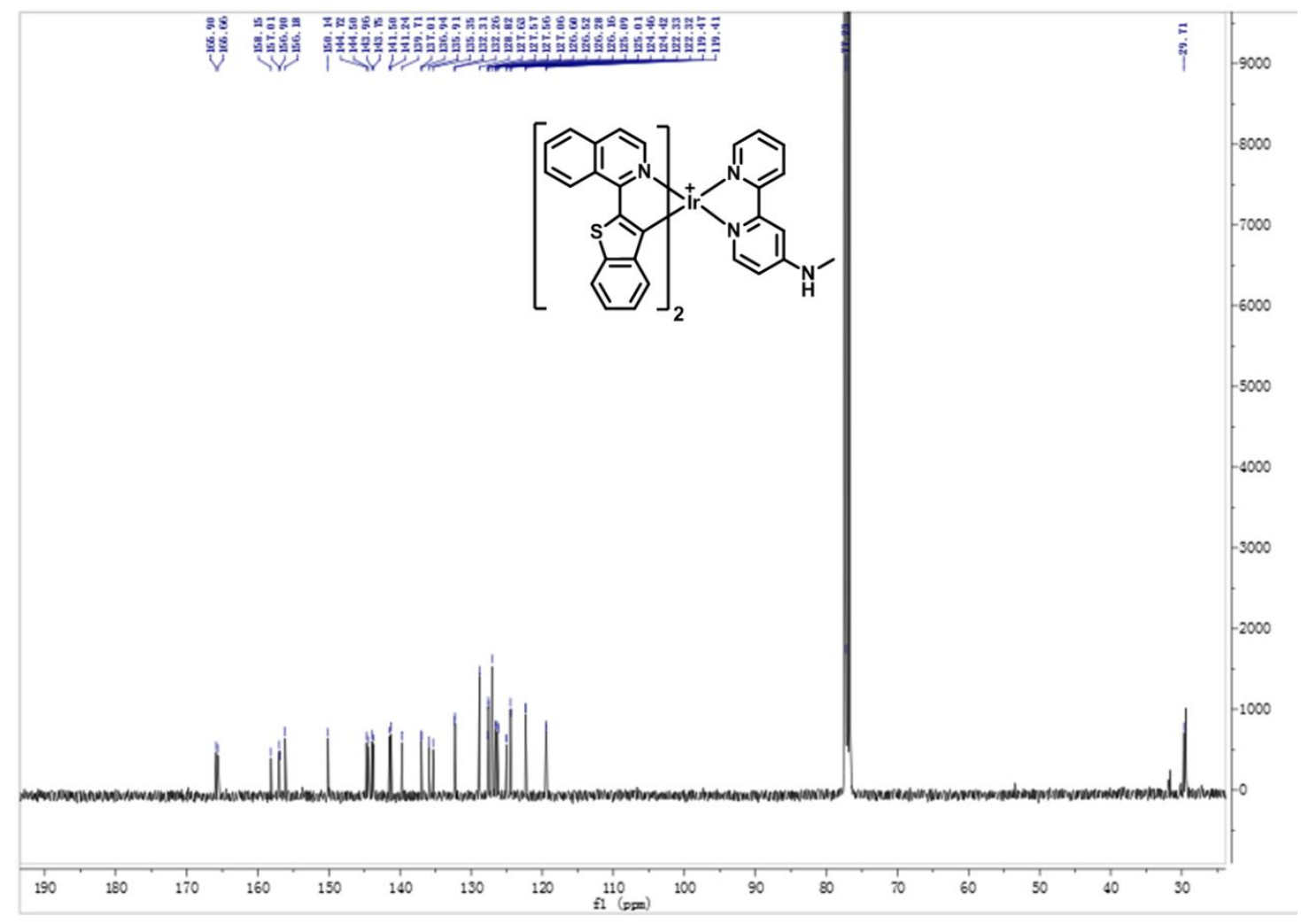

Figure S48. ${ }^{13} \mathrm{C}$ NMR spectroscopic characterization of $\mathbf{I r 4}$ * 


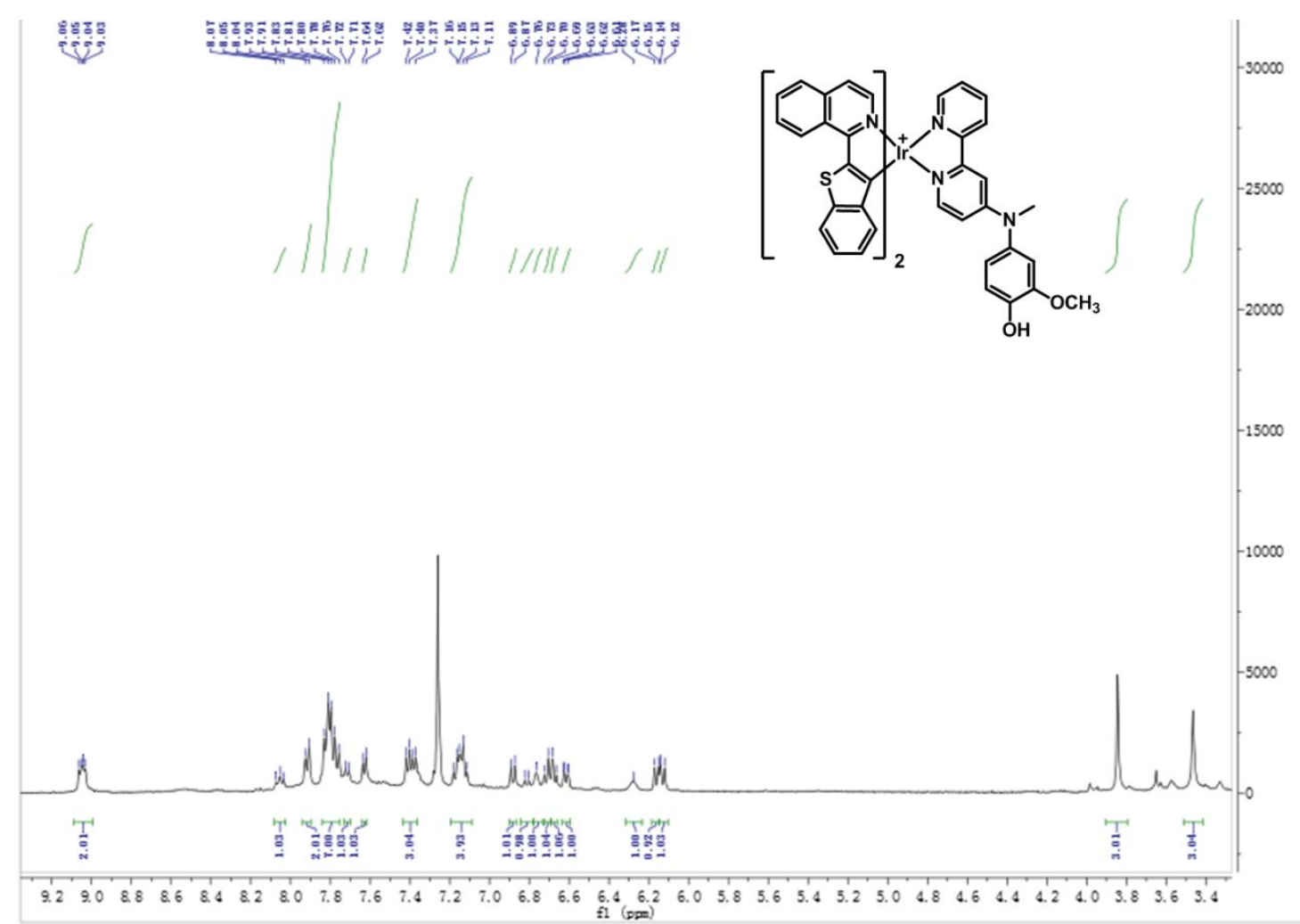

Figure S49. ${ }^{1} \mathrm{H}$ NMR spectroscopic characterization of Ir5.

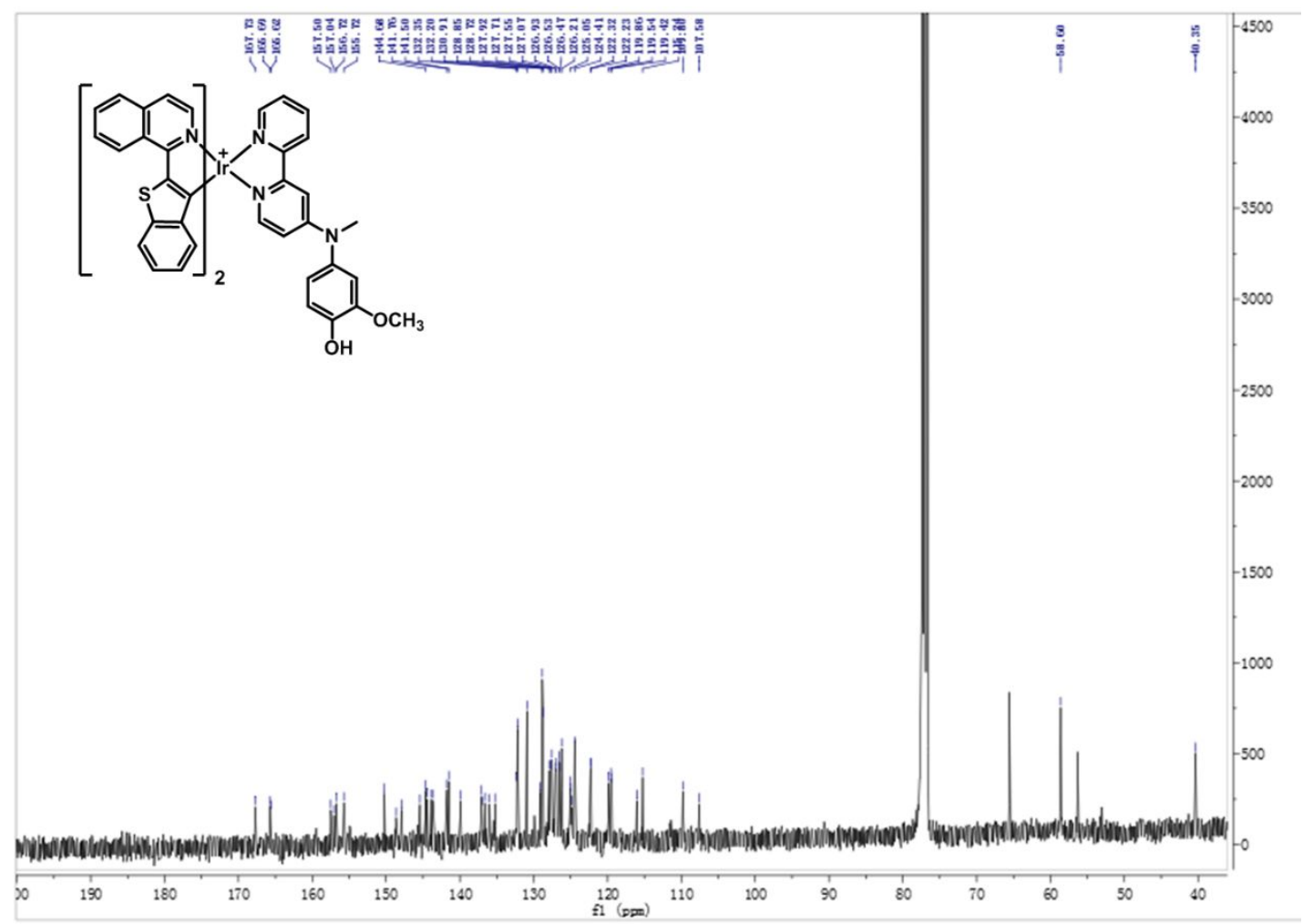

Figure S50. ${ }^{13} \mathrm{C}$ NMR spectroscopic characterization of Ir5. 


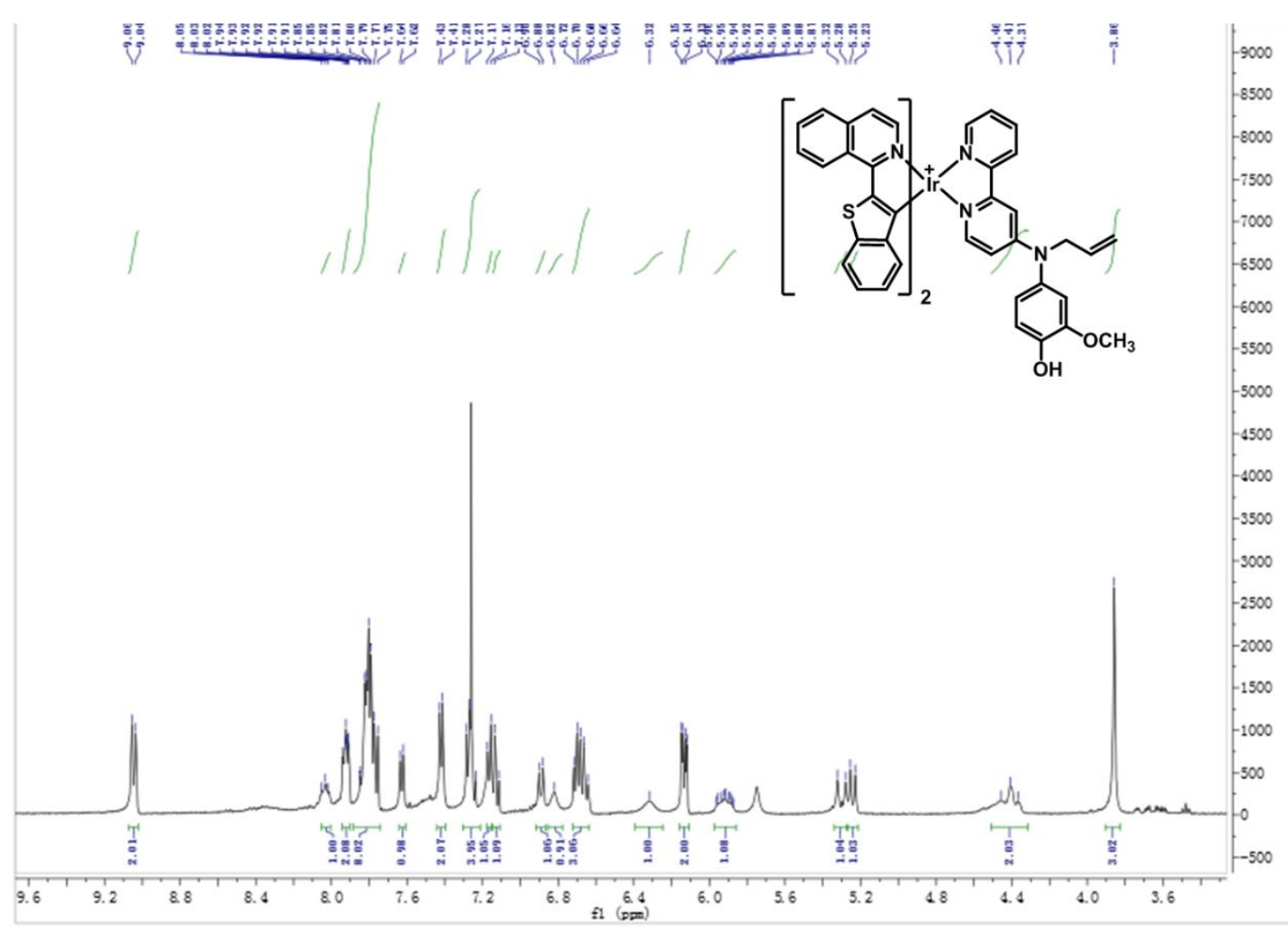

Figure S51. ${ }^{1} \mathrm{H}$ NMR spectroscopic characterization of Ir6.

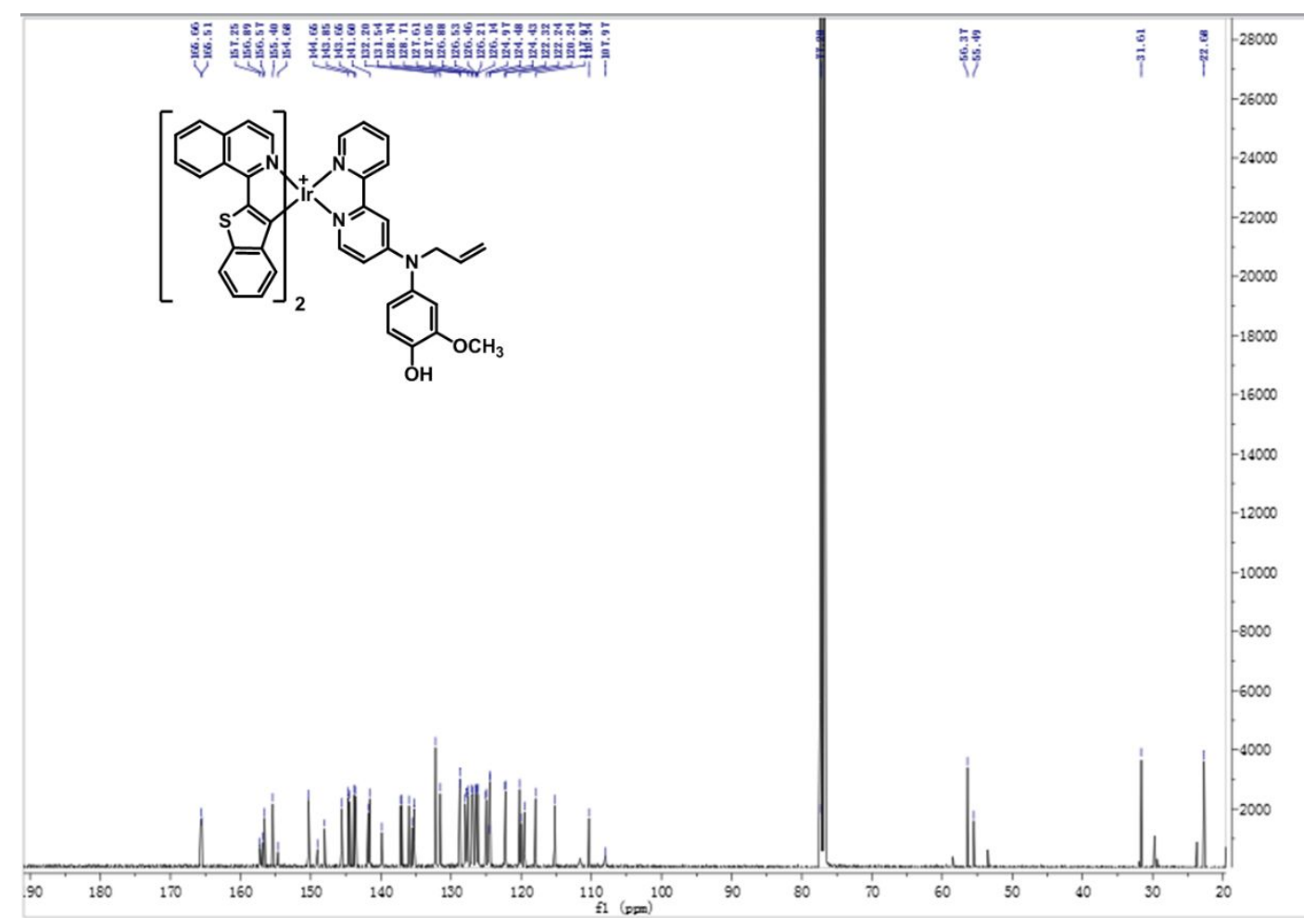

Figure S52. ${ }^{13} \mathrm{C}$ NMR spectroscopic characterization of Ir6. 
NMR spectroscopic characterization of other chemical intermediates

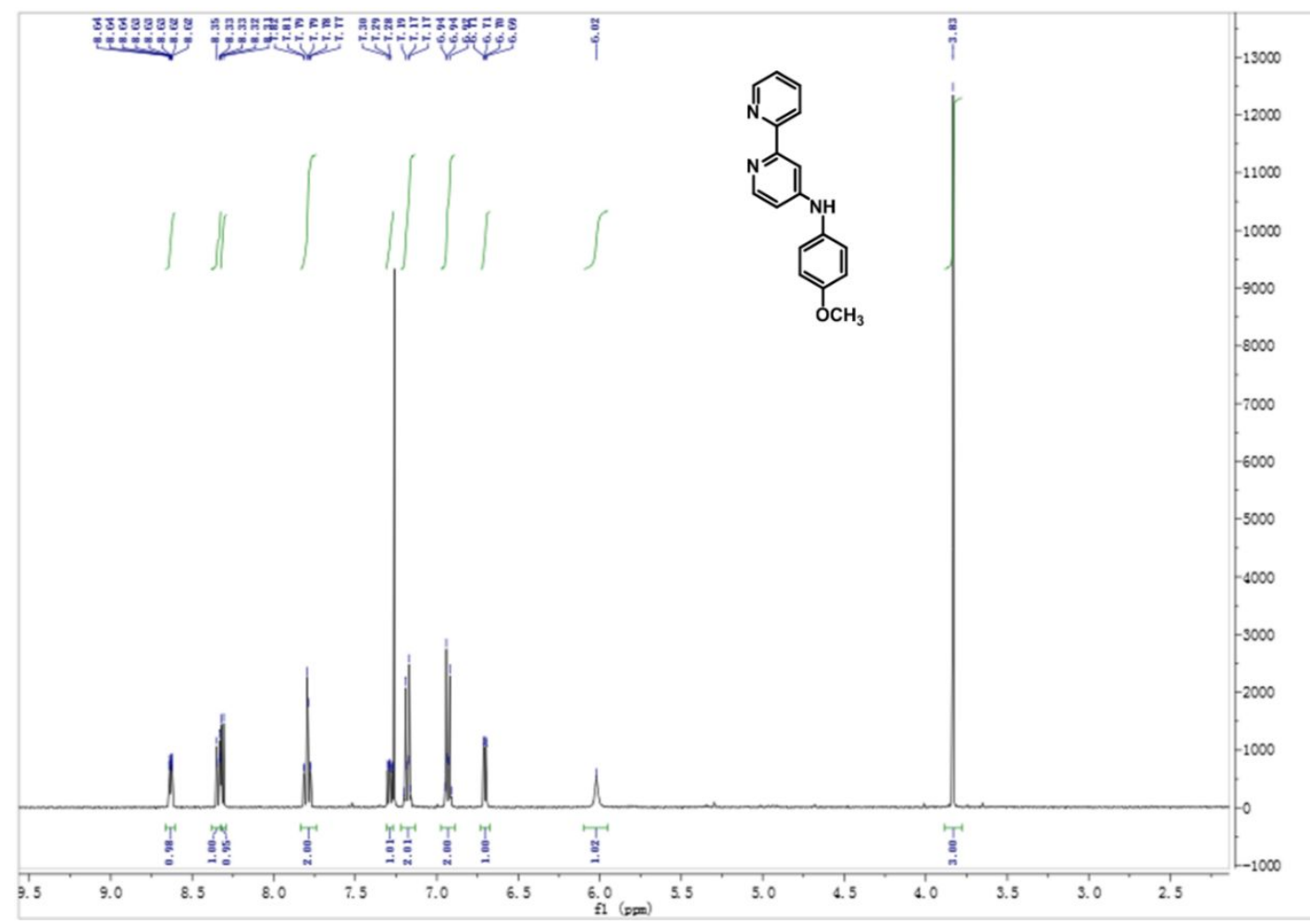

Figure S53. ${ }^{1} \mathrm{H}$ NMR spectroscopic characterization of N-(4-methoxyphenyl)-[2,2'-bipyridin]4-amine.

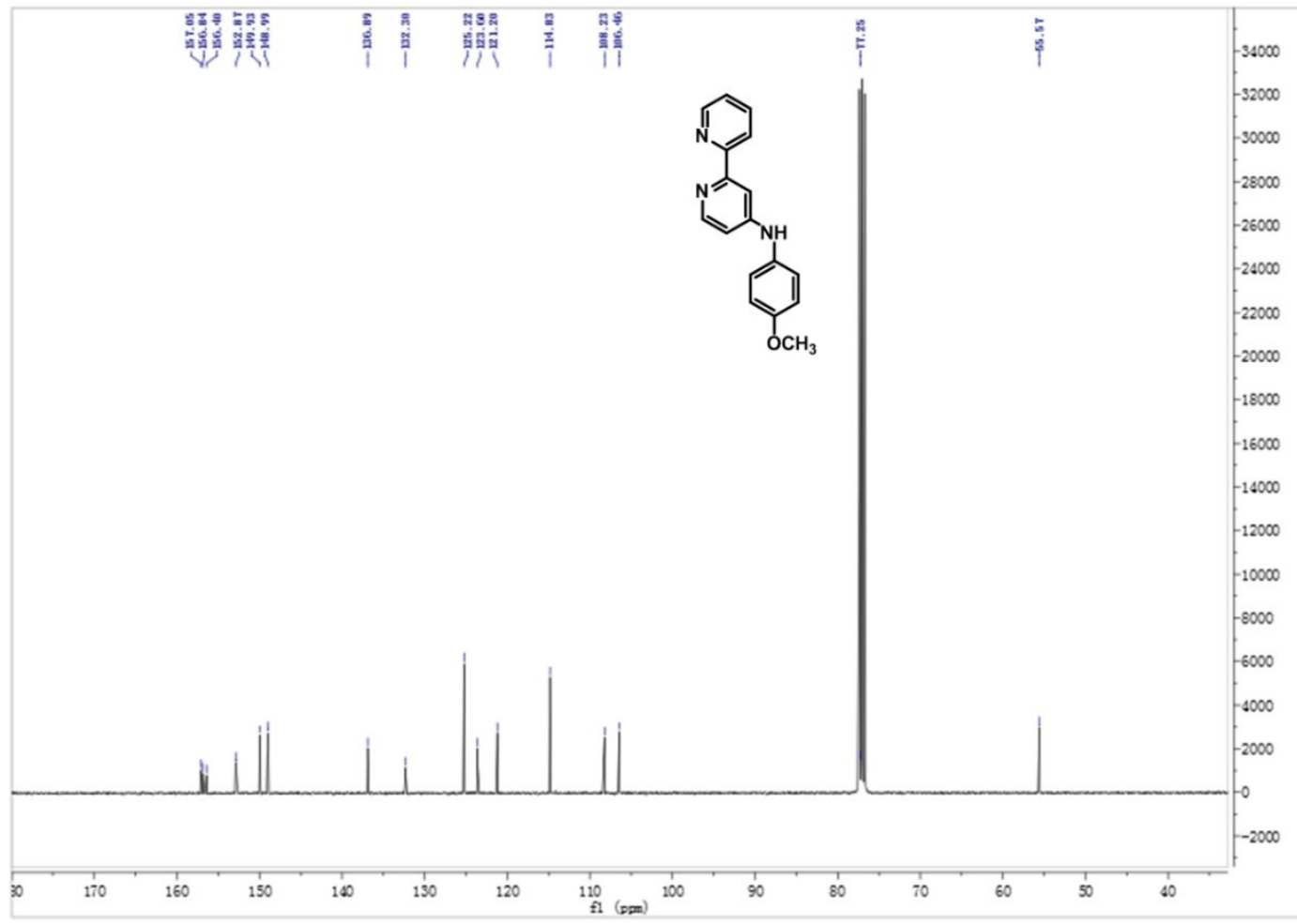

Figure S54. ${ }^{13} \mathrm{C}$ NMR spectroscopic characterization of N-(4-methoxyphenyl)-[2,2'-bipyridin]4-amine. 


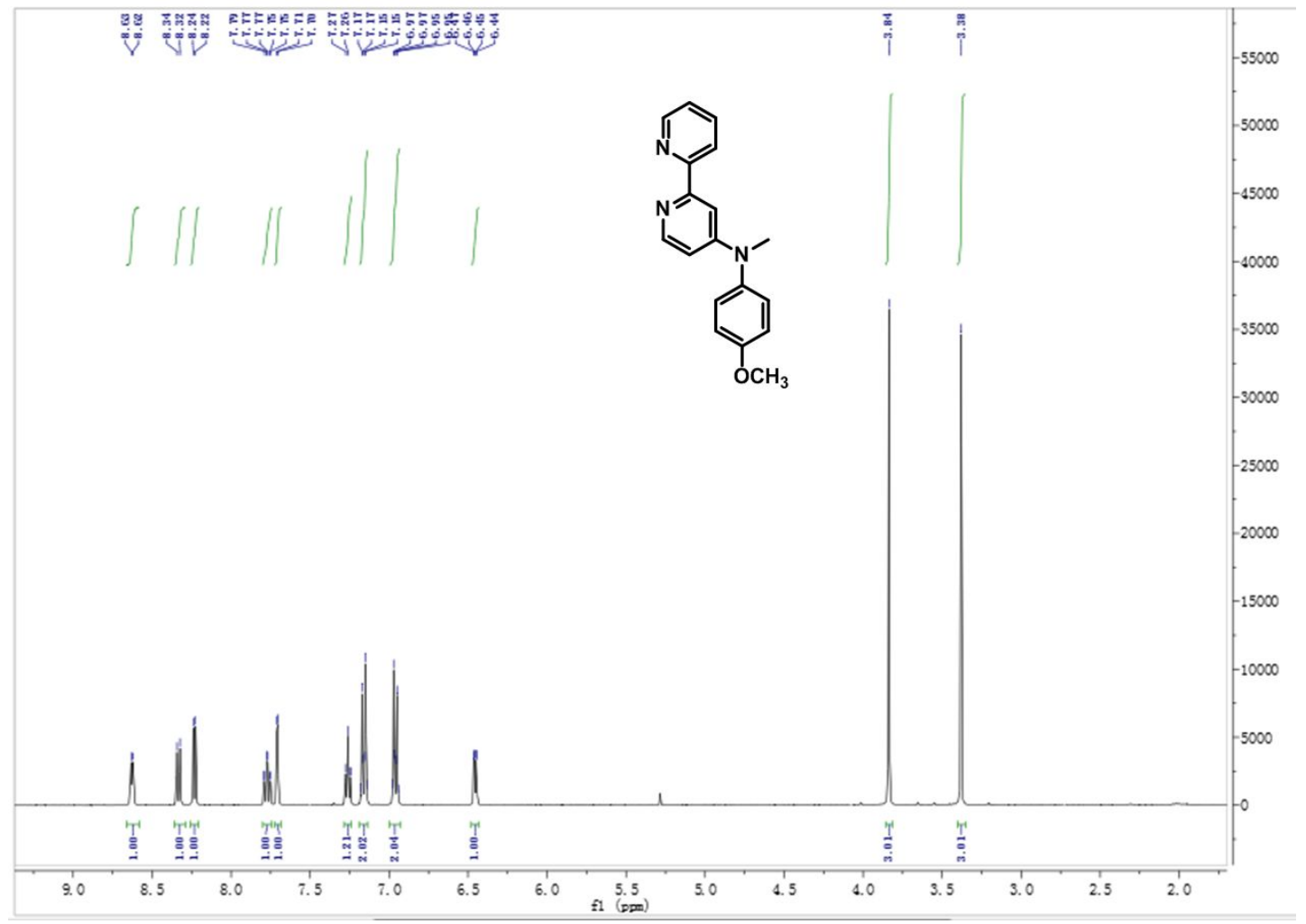

Figure S55. ${ }^{1} \mathrm{H}$ NMR spectroscopic characterization of N-(4-methoxyphenyl)-N-methyl-[2,2'bipyridin]-4-amine.

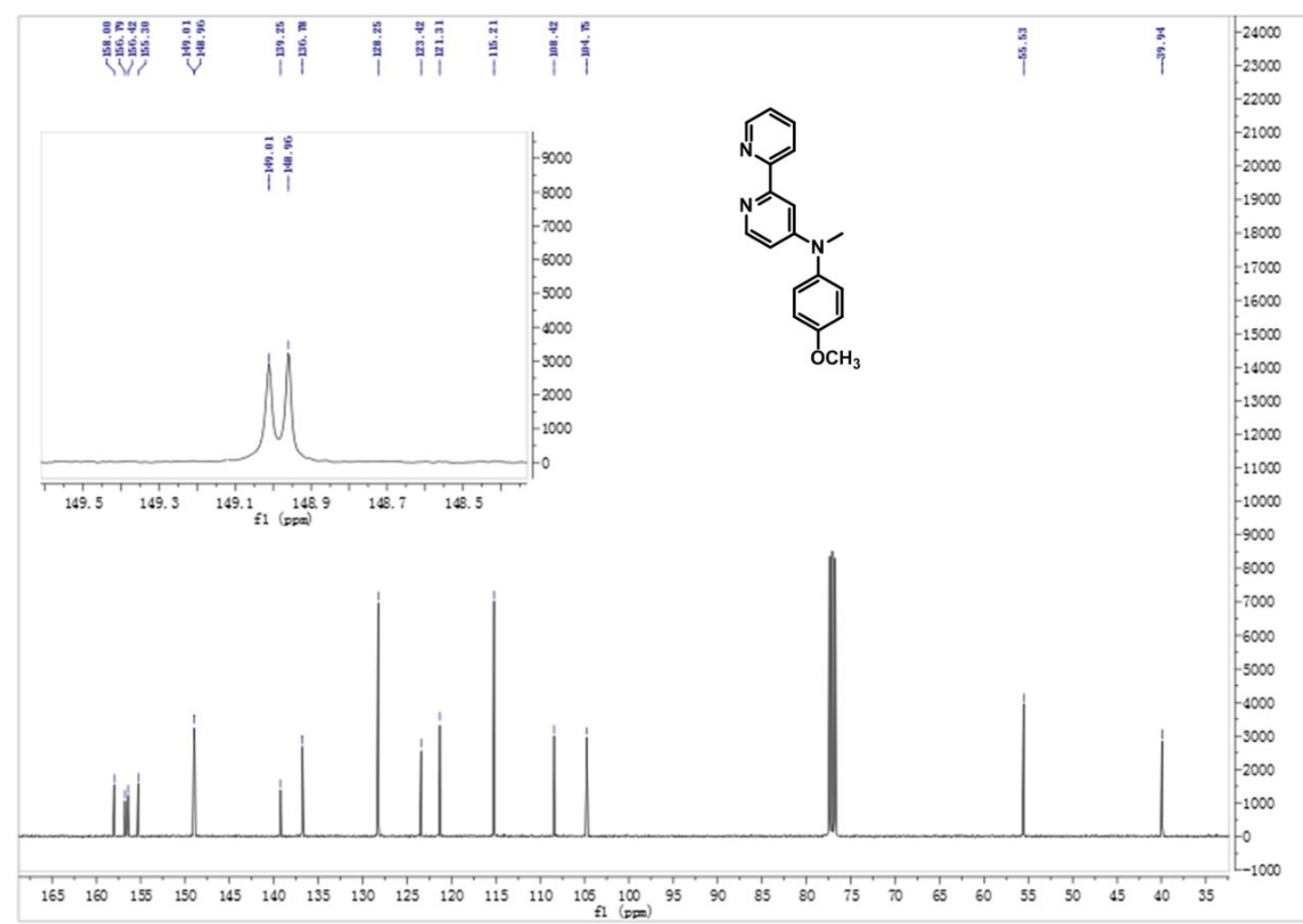

Figure S56. ${ }^{13} \mathrm{C}$ NMR spectroscopic characterization of N-(4-methoxyphenyl)-N-methyl-[2,2'bipyridin]-4-amine. 


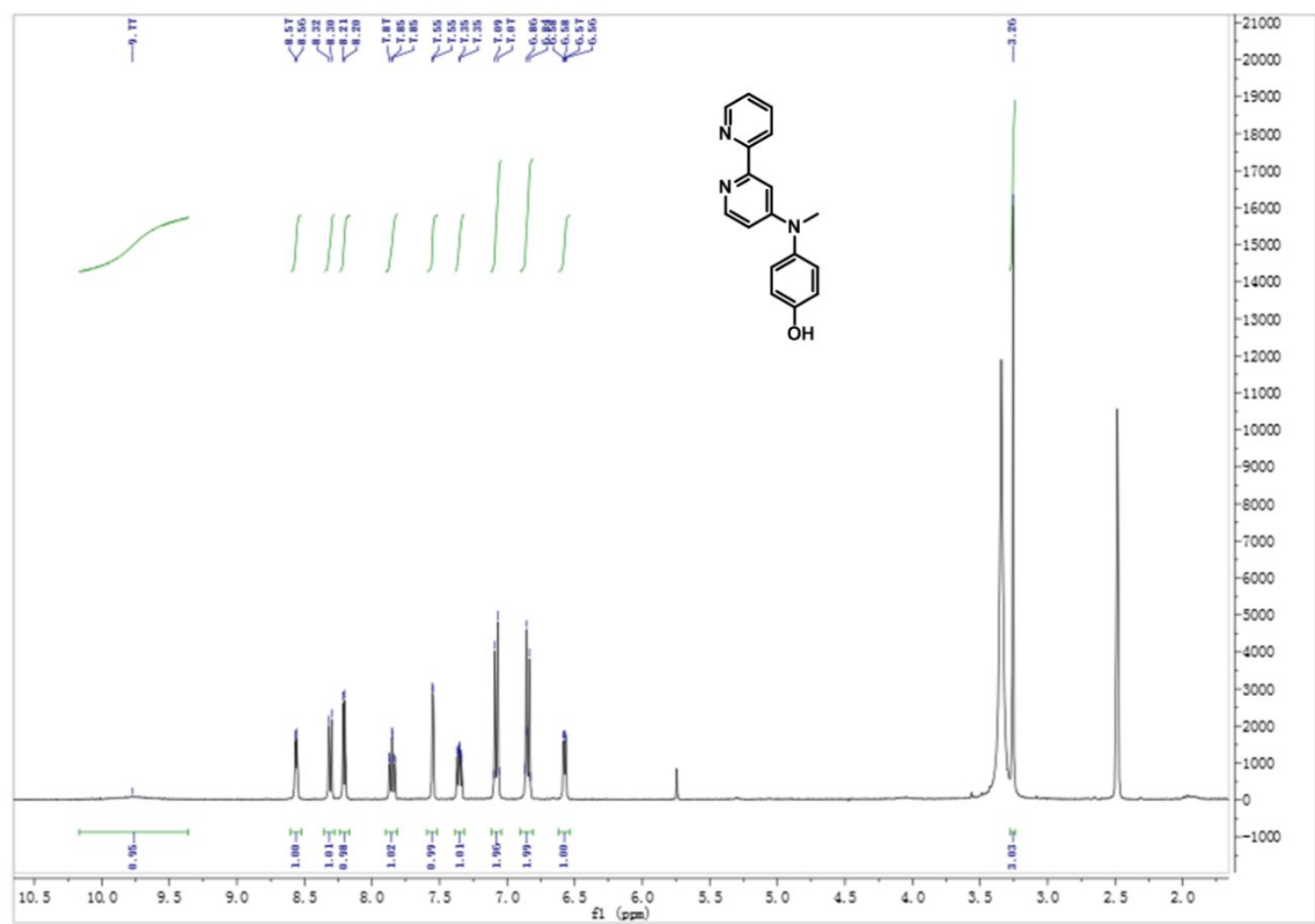

Figure S57. ${ }^{1} \mathrm{H} \quad$ NMR spectroscopic characterization of 4-([2,2'-bipyridin $]-4-$ yl(methyl)amino)phenol.

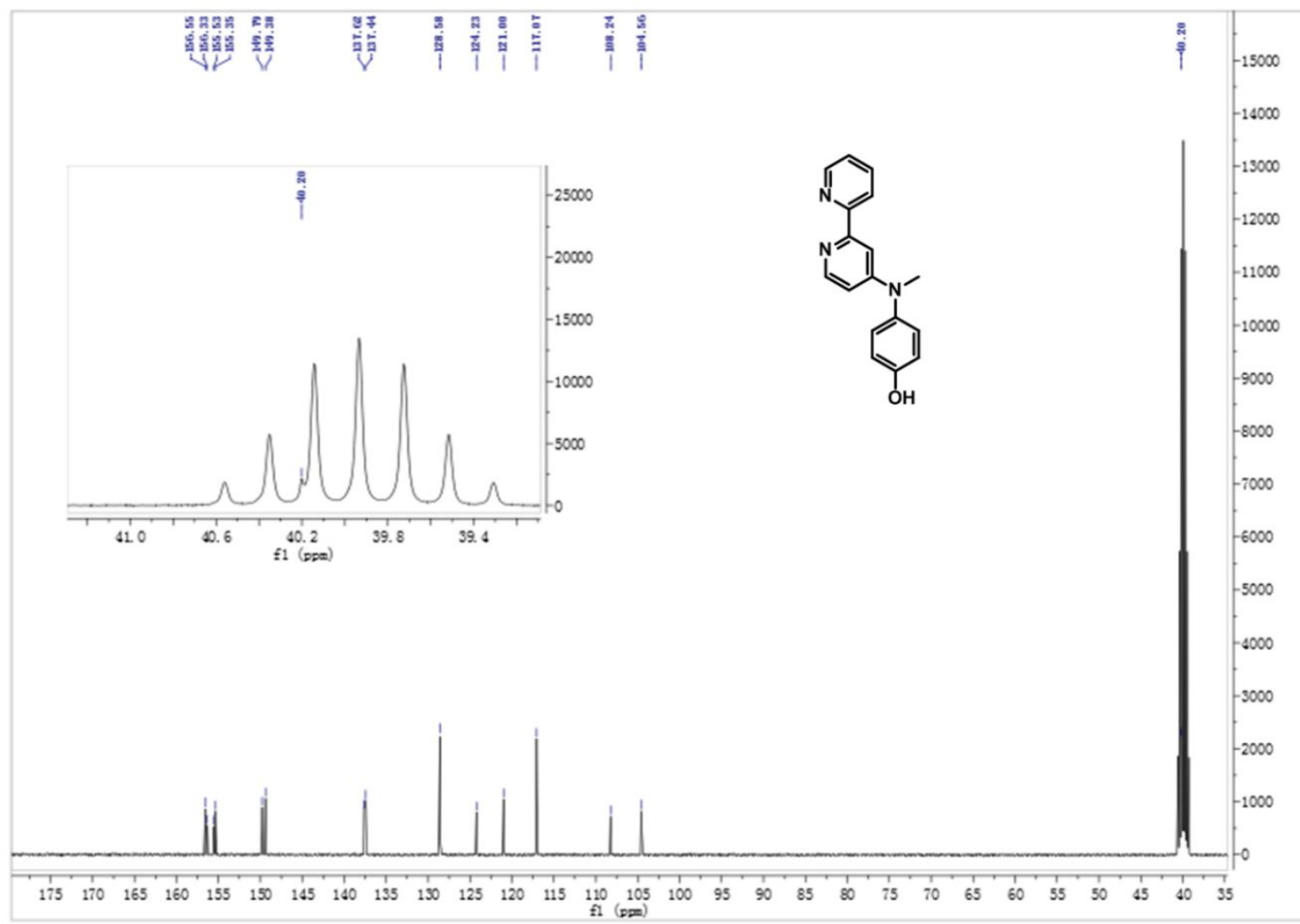

Figure S58. ${ }^{13} \mathrm{C}$ NMR spectroscopic characterization of 4-([2,2'-bipyridin]-4yl(methyl)amino)phenol. 


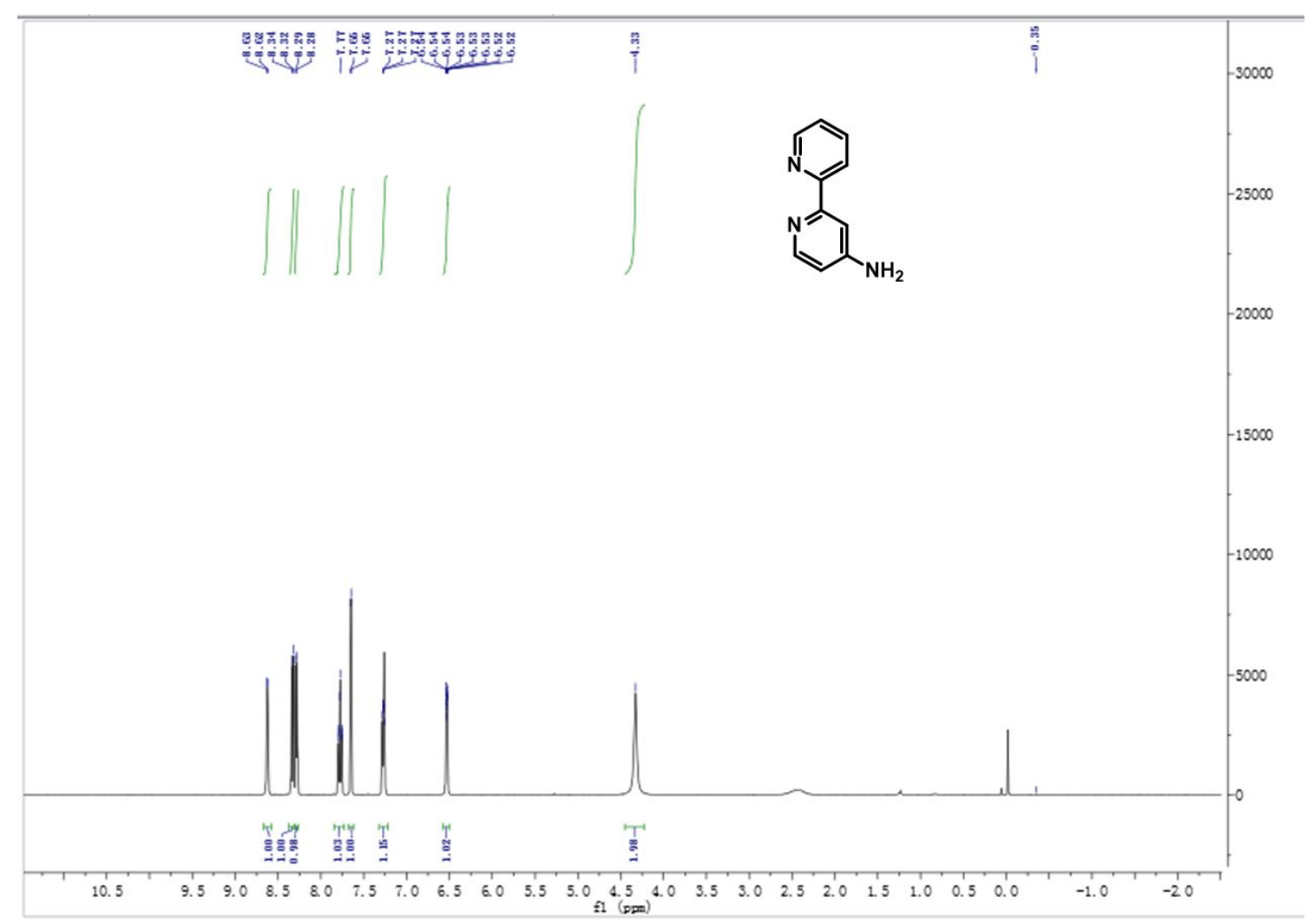

Figure S59. ${ }^{1} \mathrm{H}$ NMR spectroscopic characterization of [2,2'-bipyridin]-4-amine.

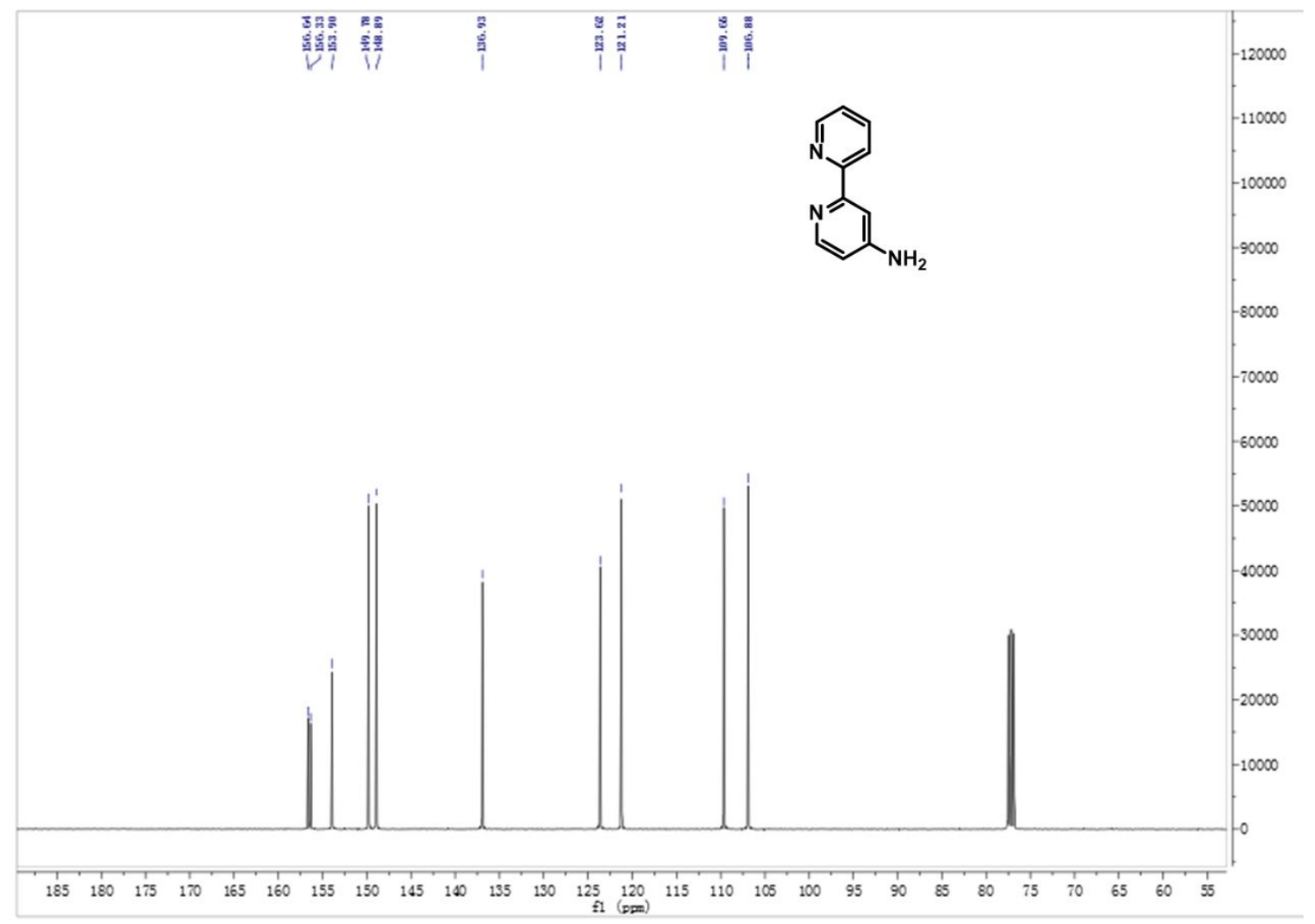

Figure S60. ${ }^{13} \mathrm{C}$ NMR spectroscopic characterization of [2,2'-bipyridin]-4-amine. 


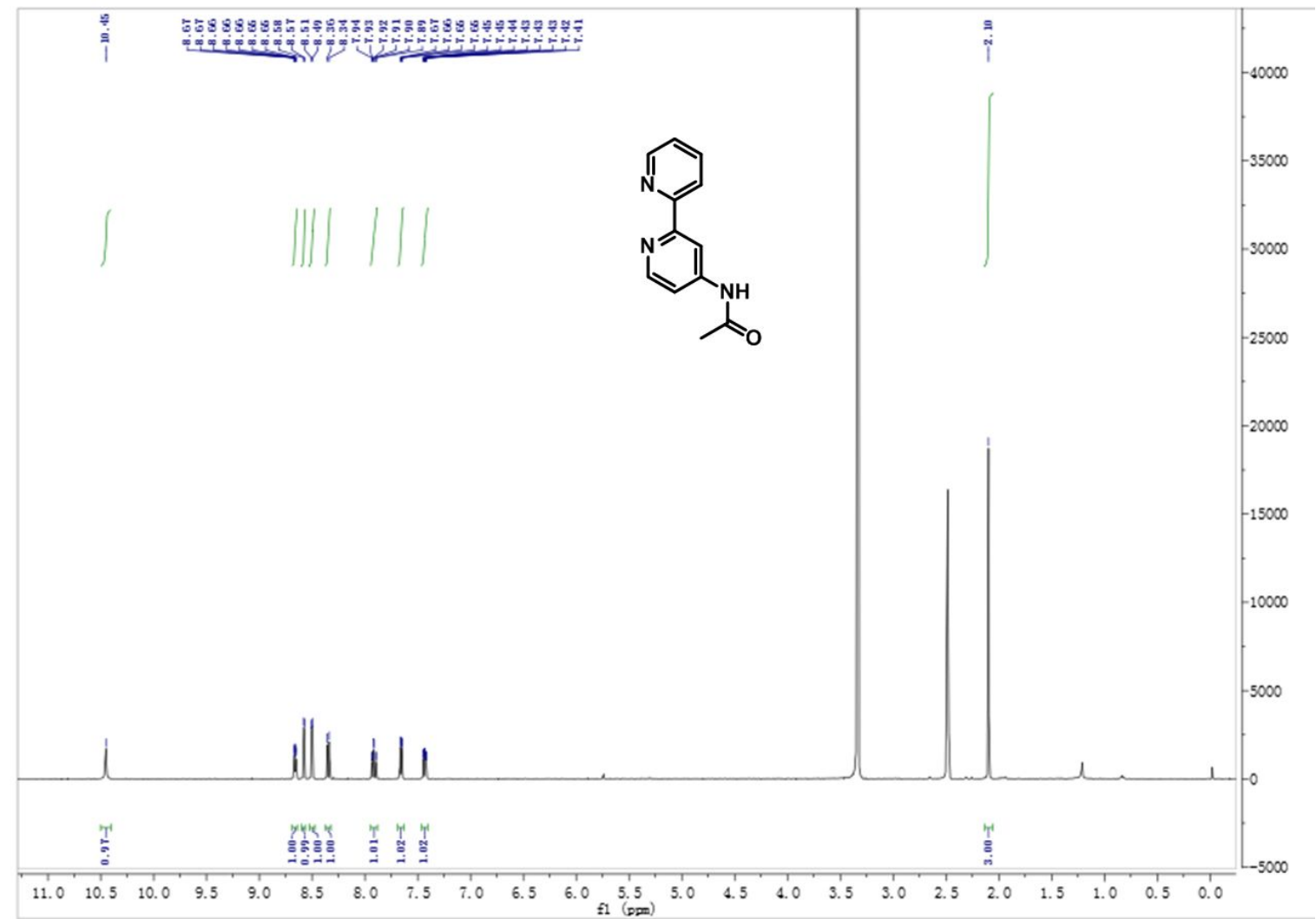

Figure S61. ${ }^{1} \mathrm{H}$ NMR spectroscopic characterization of N-([2,2'-bipyridin]-4-yl)acetamide.

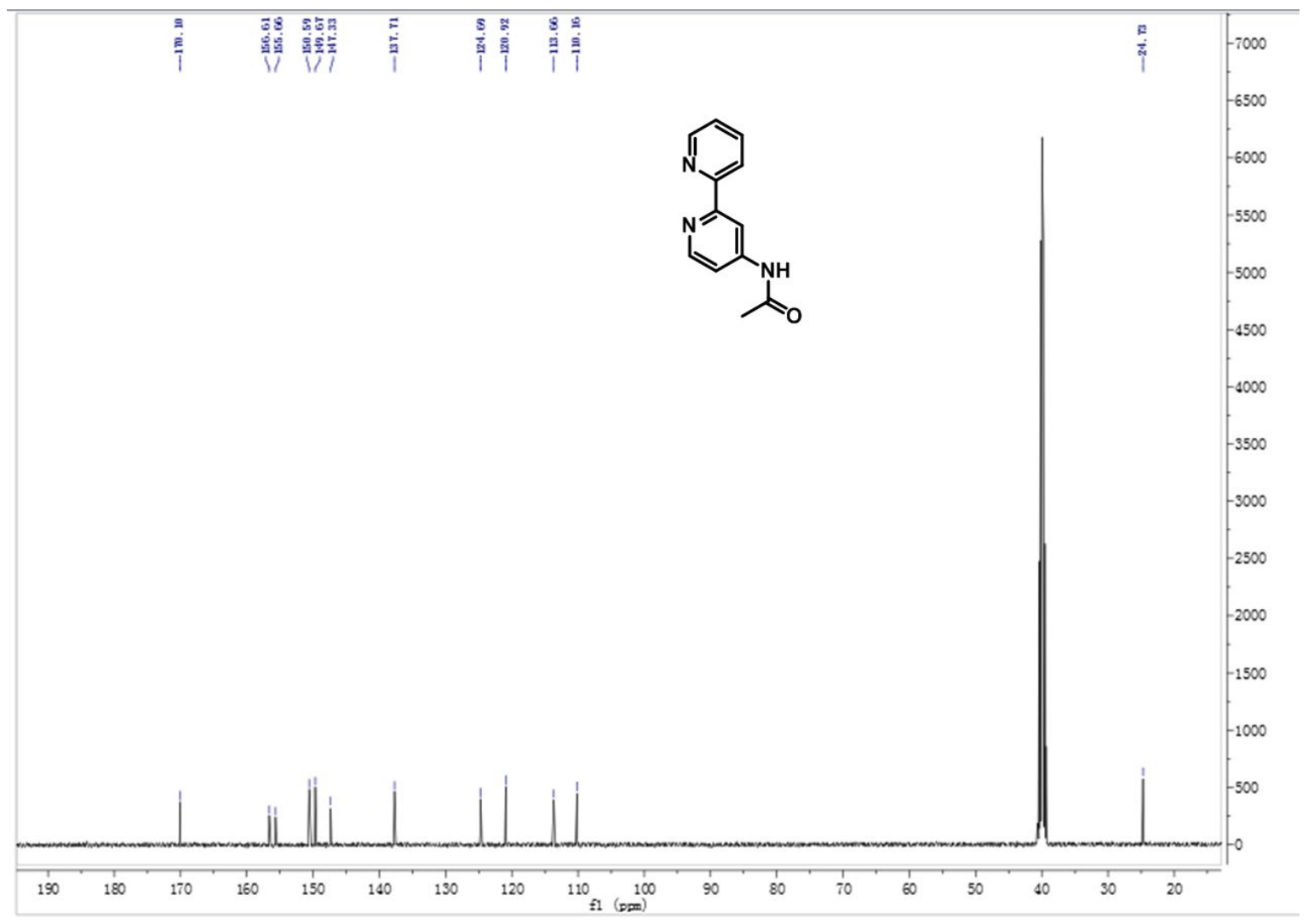

Figure S62. ${ }^{13} \mathrm{C}$ NMR spectroscopic characterization of N-([2,2'-bipyridin]-4-yl)acetamide. 


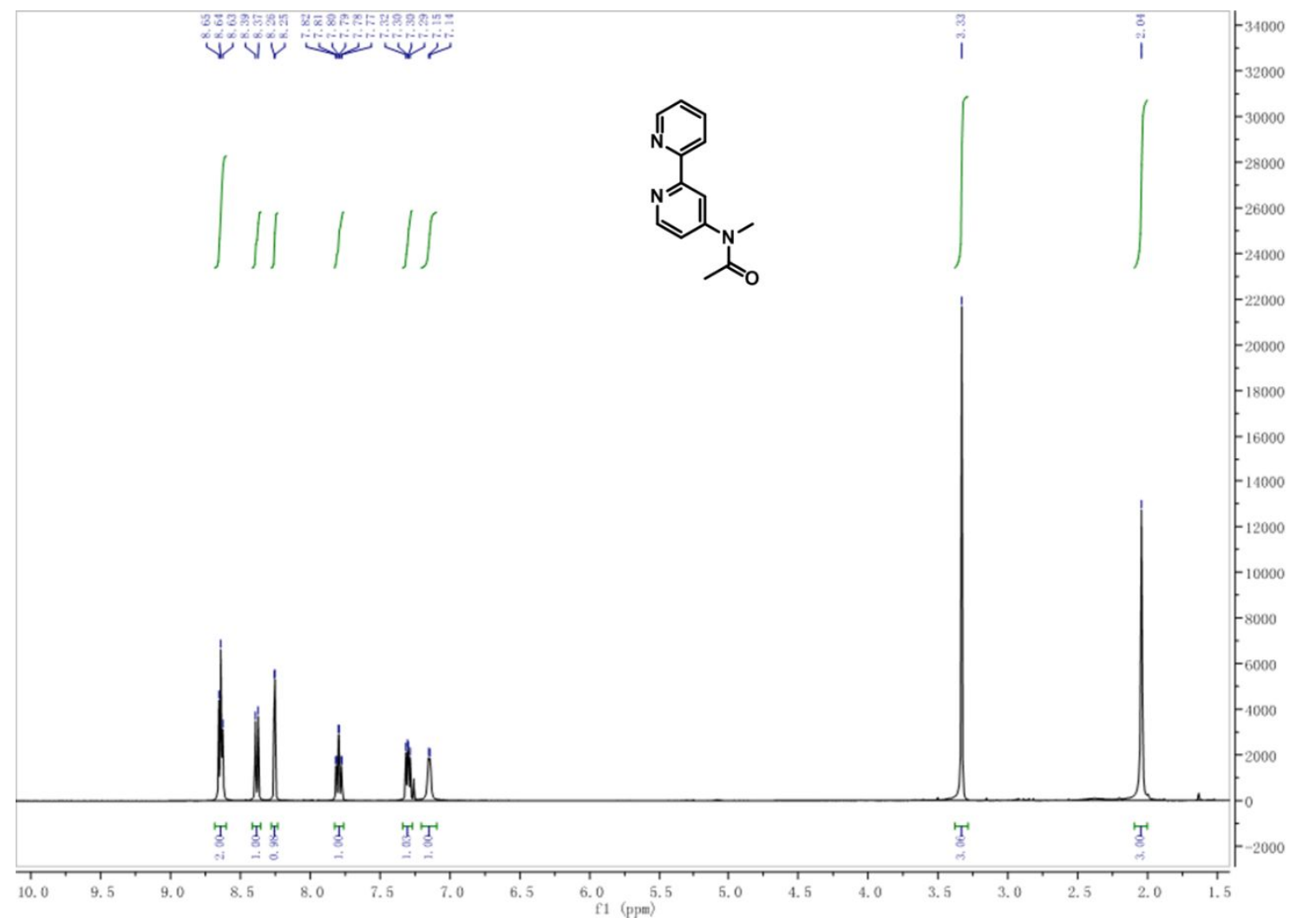

Figure S63. ${ }^{1} \mathrm{H}$ NMR spectroscopic characterization of $\mathrm{N}-\left(\left[2,2^{\prime}\right.\right.$-bipyridin $\left.]-4-\mathrm{yl}\right)-\mathrm{N}-$ methylacetamide.

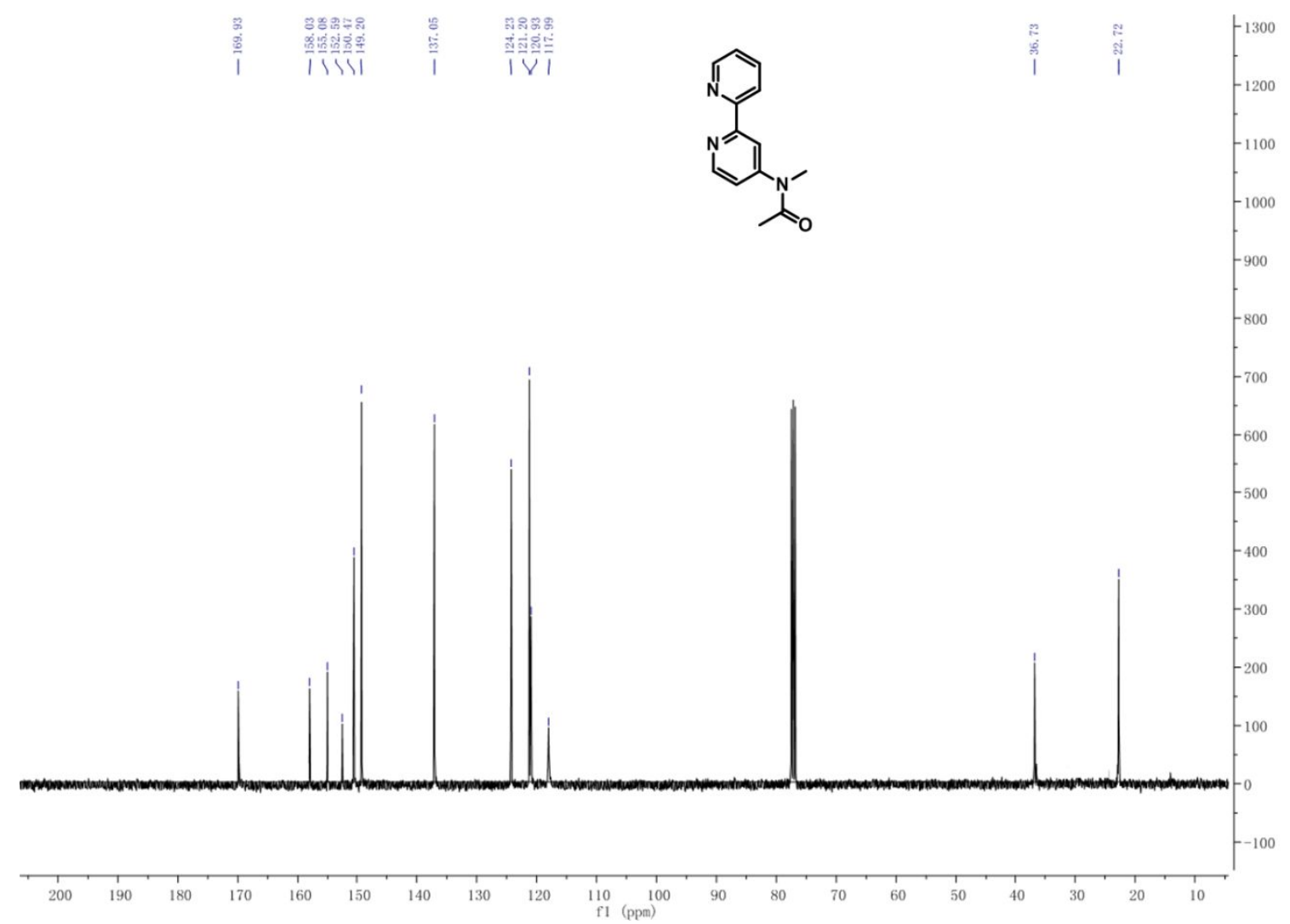

Figure S64. ${ }^{13} \mathrm{C}$ NMR spectroscopic characterization of $\mathrm{N}-\left(\left[2,2^{\prime}\right.\right.$-bipyridin]-4-yl)-Nmethylacetamide. 


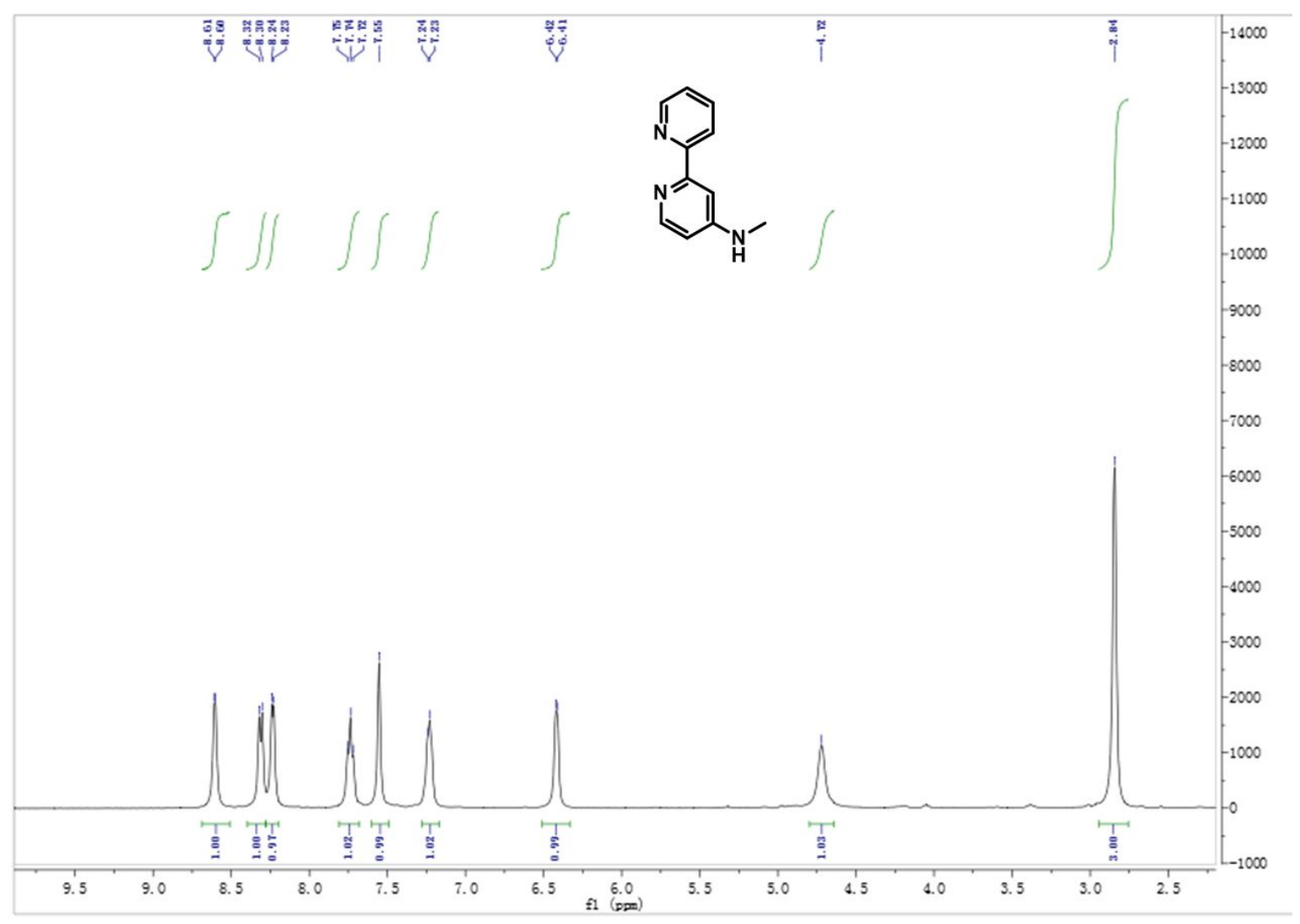

Figure S65. ${ }^{1} \mathrm{H}$ NMR spectroscopic characterization of N-methyl-[2,2'-bipyridin]-4-amine.

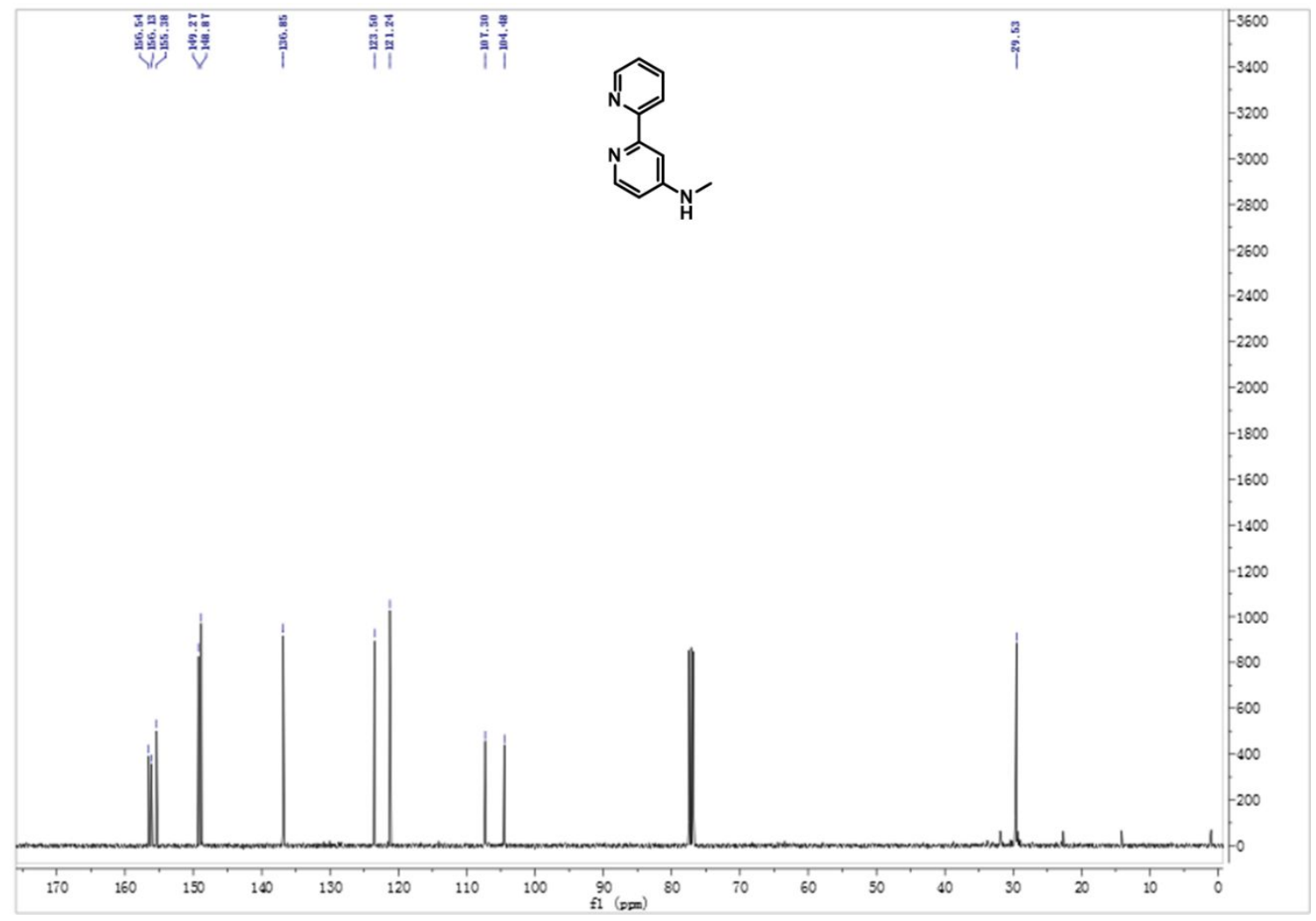

Figure S66. ${ }^{13} \mathrm{C}$ NMR spectroscopic characterization of N-methyl-[2,2'-bipyridin]-4-amine.

S-72 


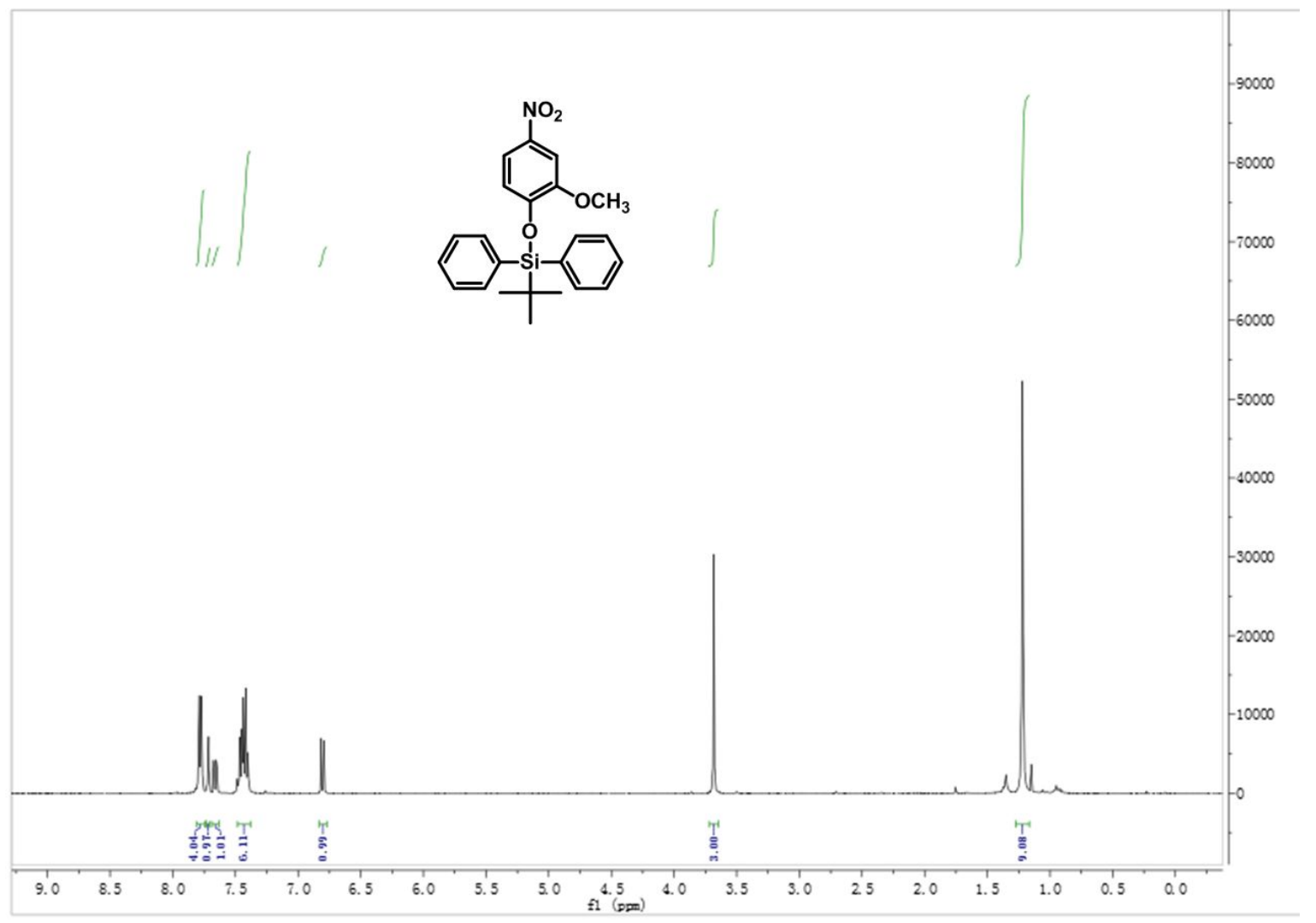

Figure S67. ${ }^{1} \mathrm{H}$ NMR spectroscopic characterization of Tert-butyl(2-methoxy-4nitrophenoxy)diphenylsilane.

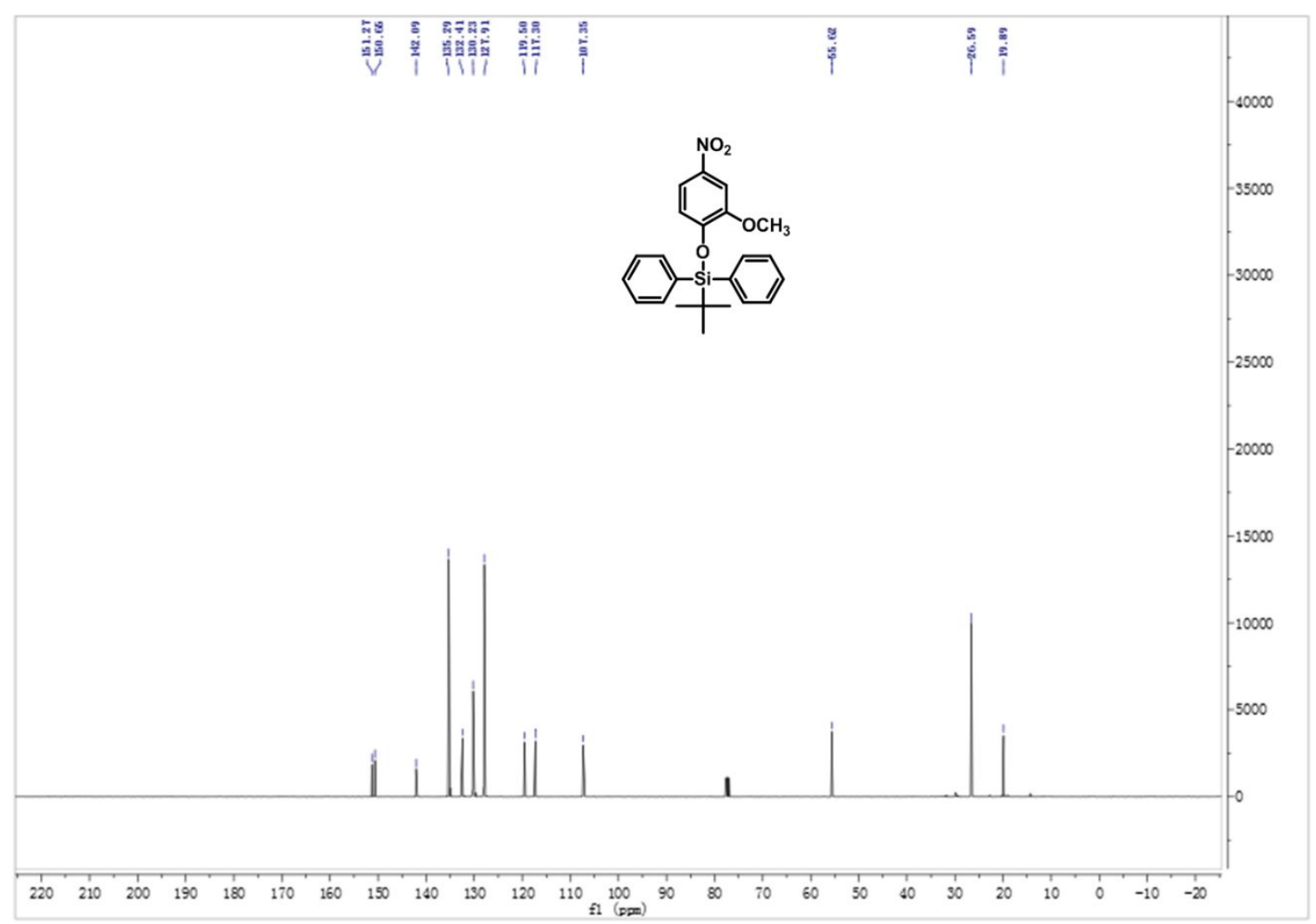

Figure S68. ${ }^{13} \mathrm{C}$ NMR spectroscopic characterization of Tert-butyl(2-methoxy-4nitrophenoxy)diphenylsilane. 


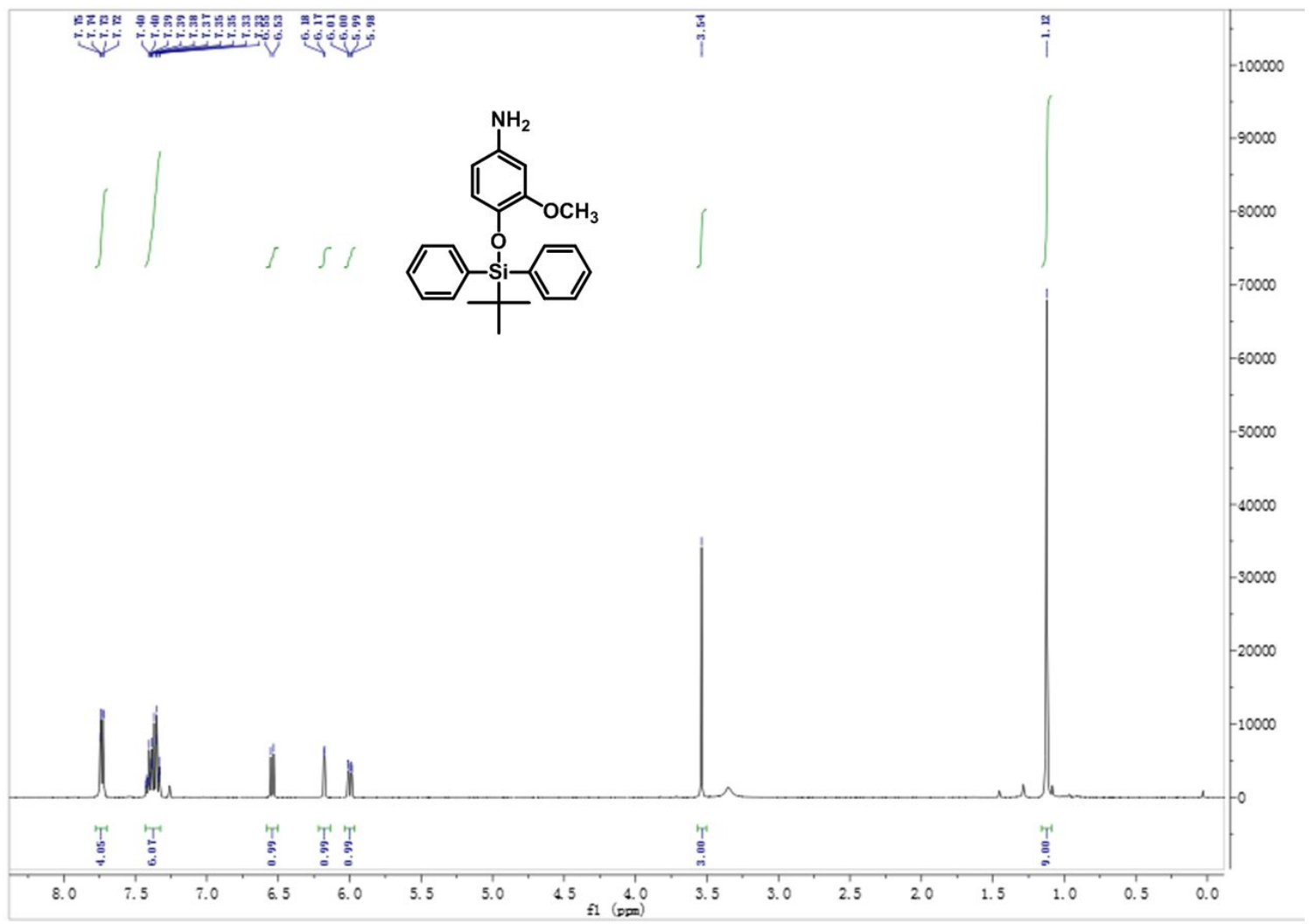

Figure S69. ${ }^{1} \mathrm{H}$ NMR spectroscopic characterization of 4-((tert-butyldiphenylsilyl)oxy)-3methoxyaniline. 


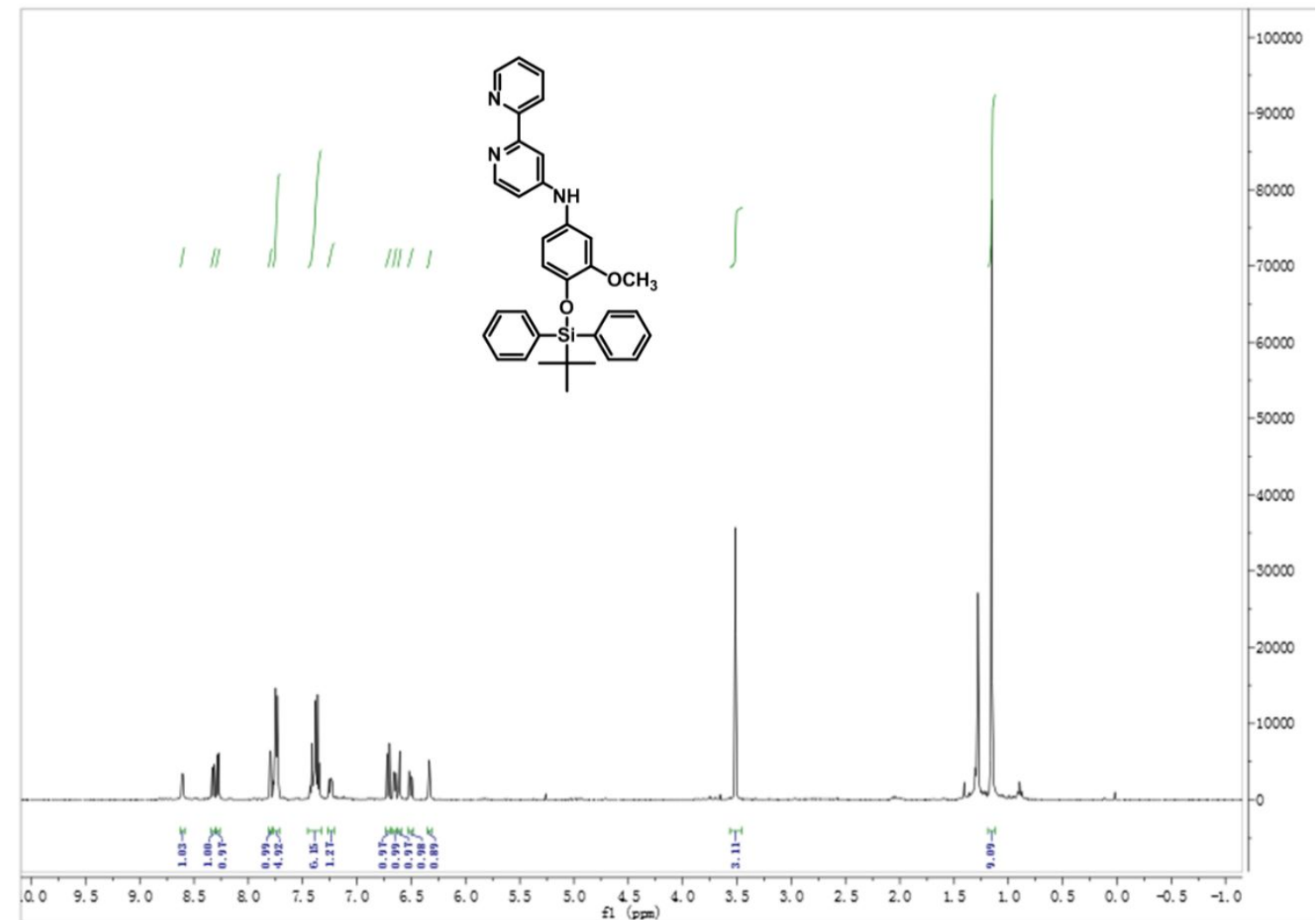

Figure S70. ${ }^{1} \mathrm{H}$ NMR spectroscopic characterization of N-(4-((tert-butyldiphenylsilyl)oxy)-3methoxyphenyl)-[2,2'-bipyridin]-4-amine.

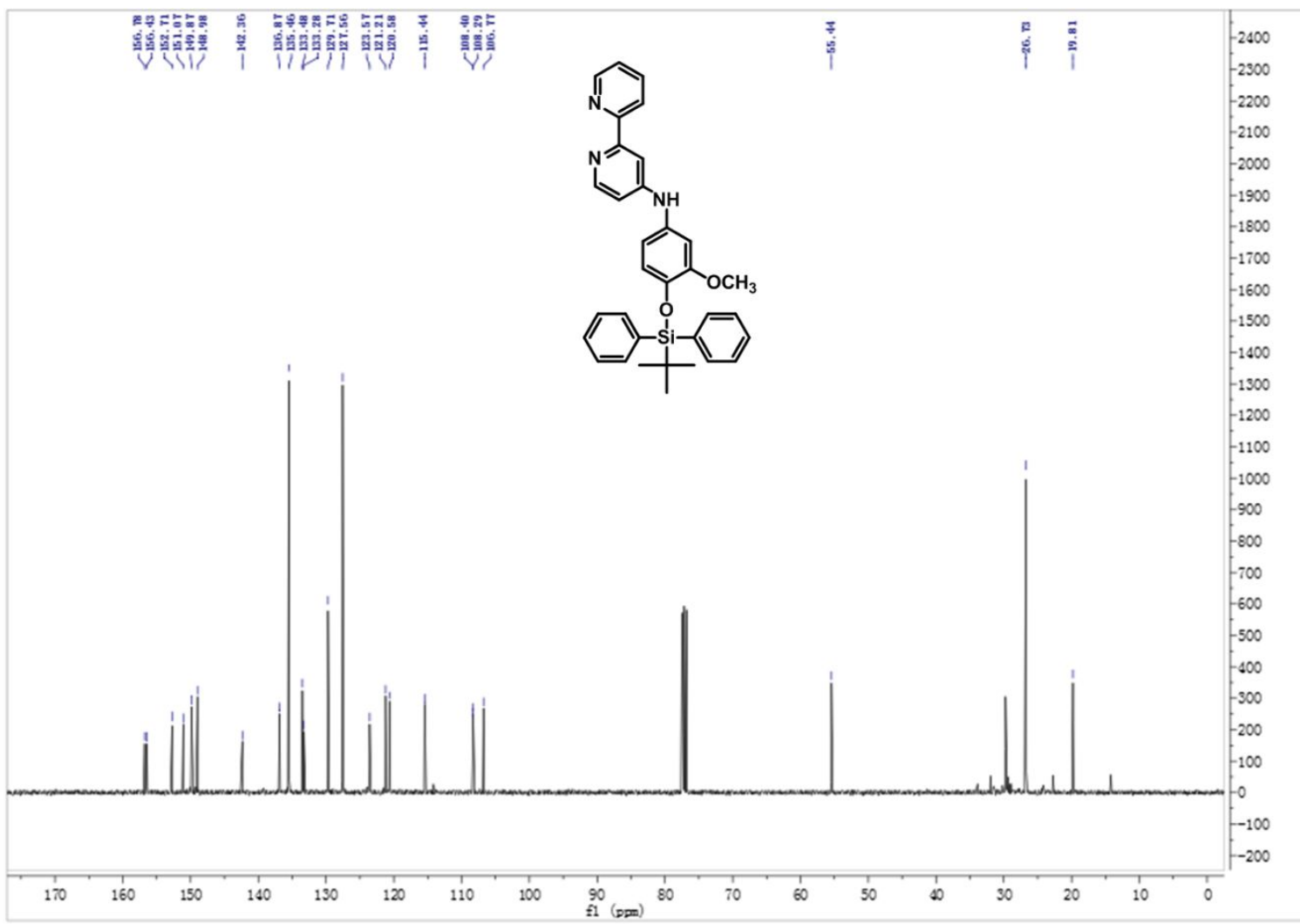

Figure S71. ${ }^{13} \mathrm{C}$ NMR spectroscopic characterization of N-(4-((tert-butyldiphenylsilyl)oxy)-3methoxyphenyl)-[2,2'-bipyridin]-4-amine. 


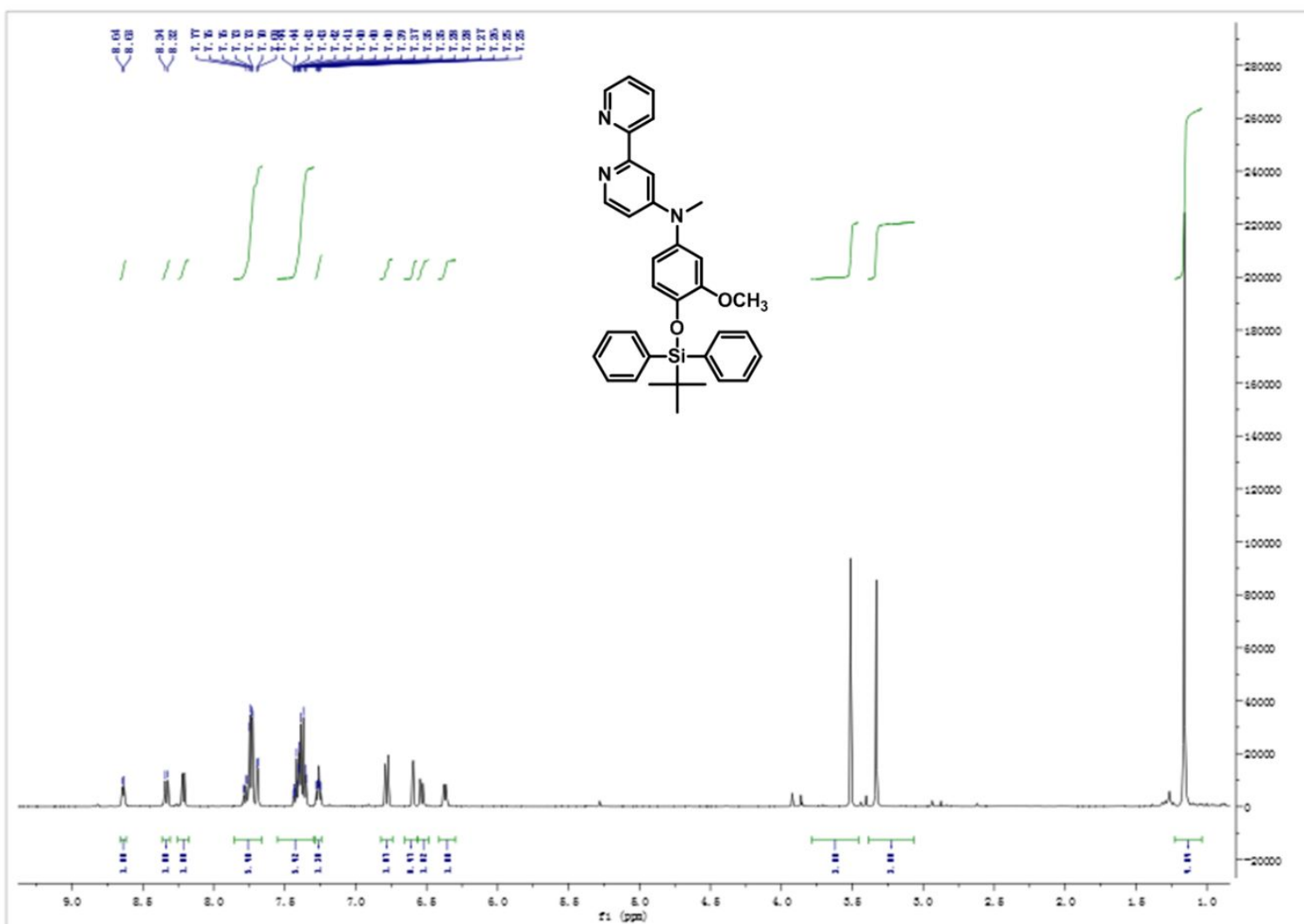

Figure S72. ${ }^{1} \mathrm{H}$ NMR spectroscopic characterization of N-(4-((tert-butyldiphenylsilyl)oxy)-3methoxyphenyl)-N-methyl-[2,2'-bipyridin]-4-amine.

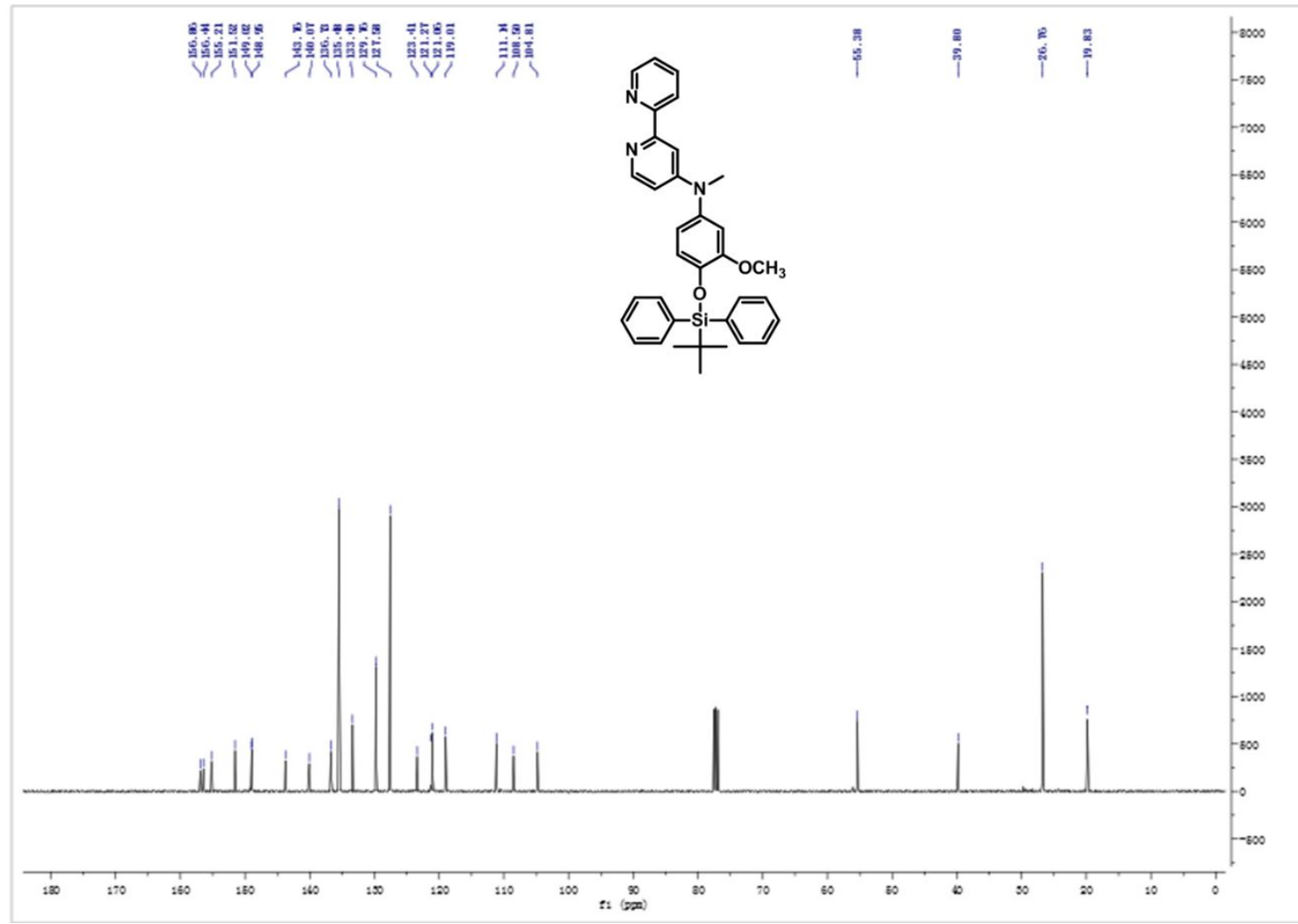

Figure S73. ${ }^{13} \mathrm{C}$ NMR spectroscopic characterization of N-(4-((tert-butyldiphenylsilyl)oxy)-3methoxyphenyl)-N-methyl-[2,2'-bipyridin]-4-amine. 


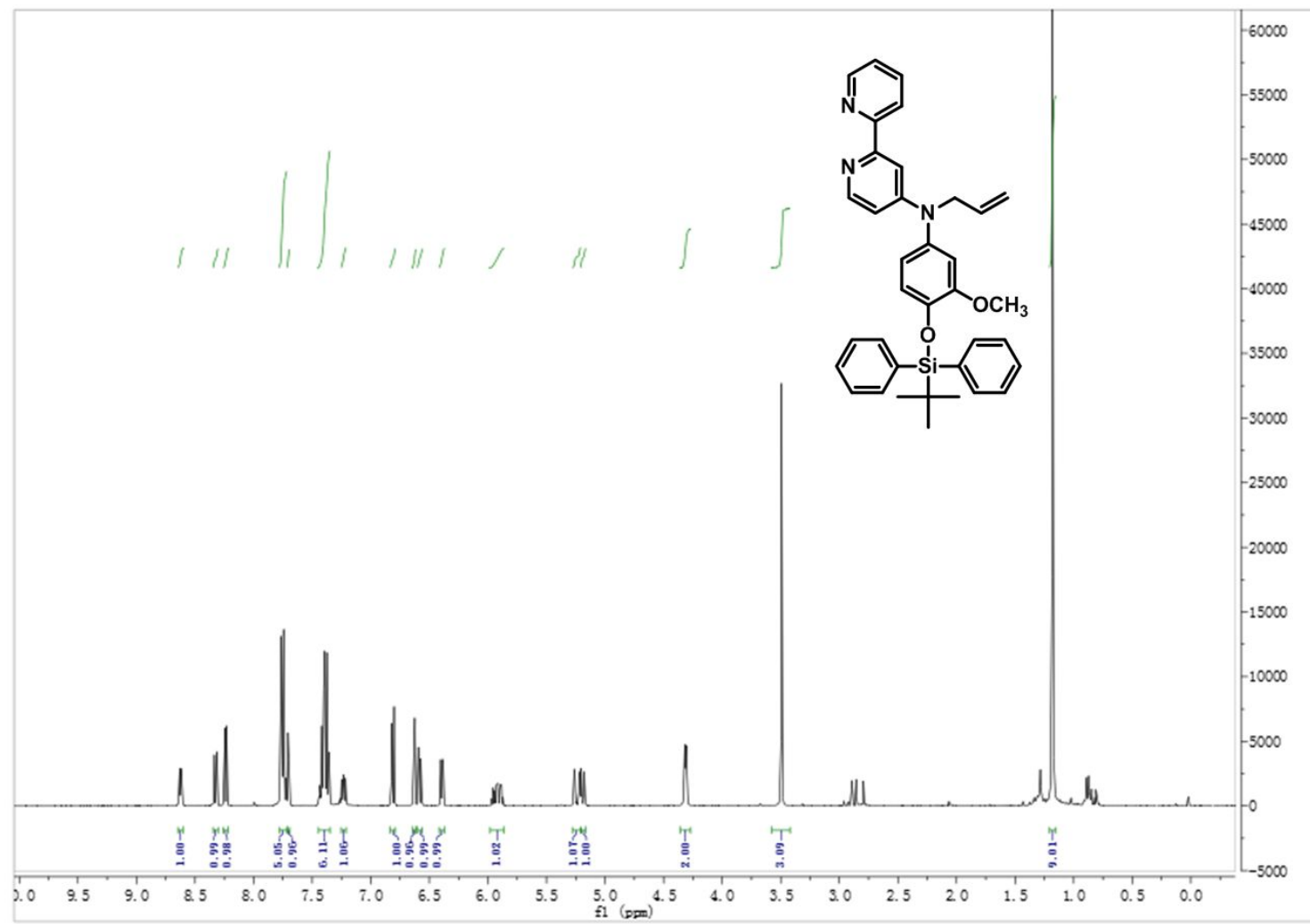

Figure S74. ${ }^{1} \mathrm{H}$ NMR spectroscopic characterization of N-allyl-N-(4-((tertbutyldiphenylsilyl)oxy)-3-methoxyphenyl)-[2,2'-bipyridin]-4-amine.

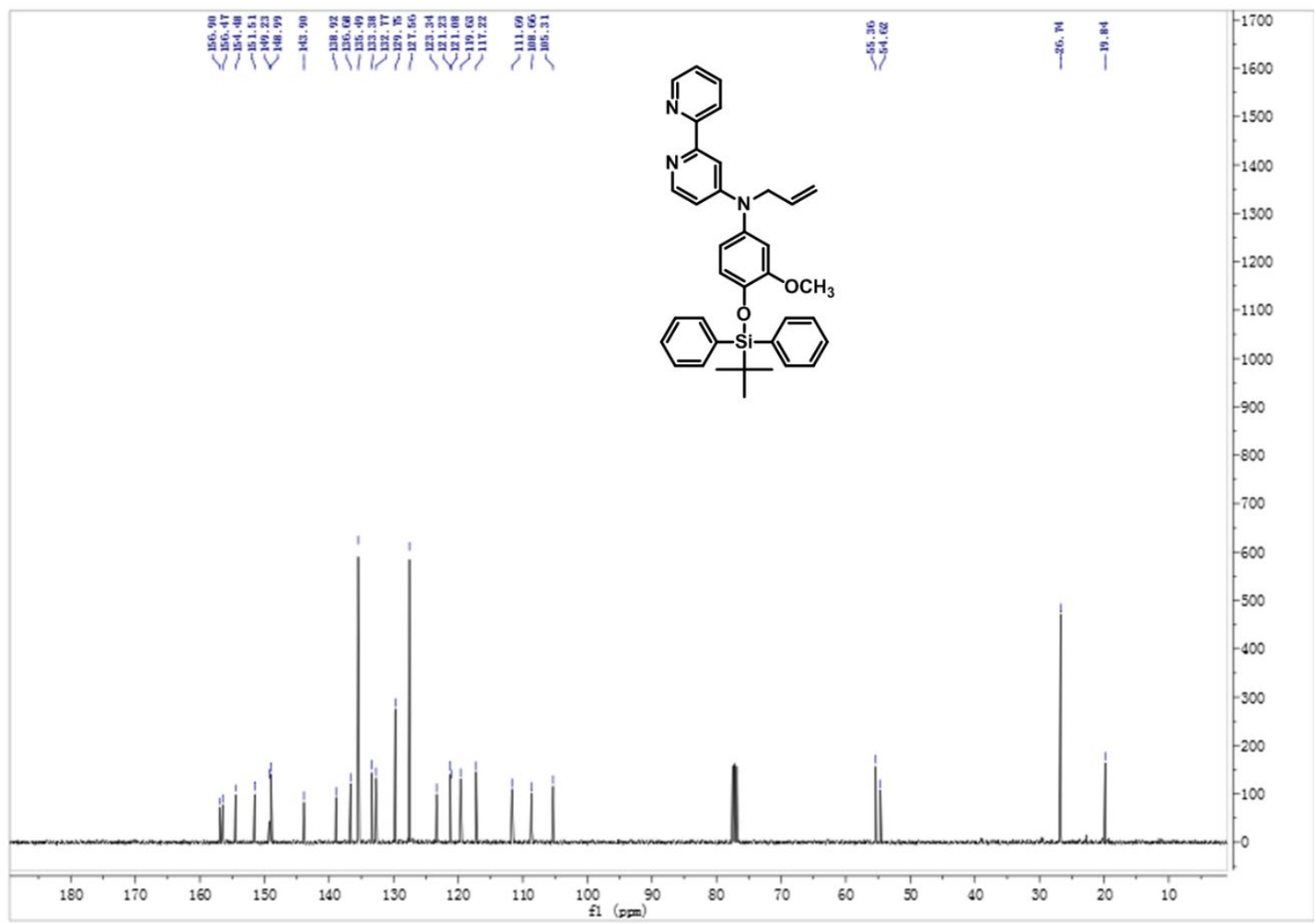

Figure S75. ${ }^{13} \mathrm{C}$ NMR spectroscopic characterization of N-allyl-N-(4-((tertbutyldiphenylsilyl)oxy)-3-methoxyphenyl)-[2,2'-bipyridin]-4-amine. 


\section{References}

[1] W. L. F. Armarego, C. L. L. Chai, Purification of Laboratory Chemicals, 6th ed., Elsevier, Oxford, 2009.

[2] M. Zalas, B. Gierczyk, M. Ceglowski, G. Schroeder, Synthesis of new dendritic antennalike polypyridine ligands, Chem. Pap. 66 (2012) 733. DOI: 10.2478/s11696-012-0196-5

[3] M. B. Nonoyama, Benzo[h]quinolin-10-yl-N iridium(III) complexes, Bull. Chem. Soc. Jpn. 47 (1974) 767-768. DOI: 10.1246/bcsj.47.767

[4] Z. Chen, K. Y. Zhang, X. Tong, Y. Liu, C. Hu, S. Liu, Q. Yu, Q. Zhao, W. Huang, Phosphorescent polymeric thermometers for in vitro and in vivo temperature sensing with minimized background interference, Adv. Funct. Mater. 26 (2016) 4386-4396. DOI: 10.1002/adfm.201600706 